NBS

Eechnical Note

$n_{0.18-22}$

\title{
QUARTERLY RADIO NOISE DATA MARCH, APRIL, MAY, 1964
}

W. Q. CRICHLOW, R. T. DISNEY, and $M$. A. JENKINS 


\section{THE NATIONAL BUREAU OF STANDARDS}

The National Bureau of Standards is a principal focal point in the Federal Government for assuring maximum application of the physical and engineering sciences to the advancement of technology in industry and commerce. Its responsibilities include development and maintenance of the national standards of measurement, and the provisions of means for making measurements consistent with those standards; determination of physical constants and properties of materials; development of methods for testing materials, mechanisms, and structures, and making such tests as may be necessary, particularly for government agencies; cooperation in the establishment of standard practices for incorporation in codes and specifications; advisory service to government agencies on scientific and technical problems; invention and development of devices to serve special needs of the Government; assistance to industry, business, and consumers in the development and acceptance of commercial standards and simplified trade practice recommendations; administration of programs in cooperation with United States business groups and standards organizations for the development of international standards of practice; and maintenance of a clearinghouse for the collection and dissemination of scientific, technical, and engineering information. The scope of the Bureau's activities is suggested in the following listing of its four Institutes and their organizational units.

Institute for Basic Standards. Applied Mathematics. Electricity. Metrology. Mechanics. Heat. Atomic Physics. Physical Chemistry. Laboratory Astrophysics. ${ }^{*}$ Radiation Physics. Radio Standards Laboratory:* Radio Standards Physics; Radio Standards Engineering. Office of Standard Reference Data.

Institute for Materials Research. Analytical Chemistry. Polymers. Metallurgy. Inorganic Materials. Reactor Radiations. Cryogenics." Materials Evaluation Laboratory. Office of Standard Reference Materials.

Institute for Applied Technology. Building Research. Information Technology. Performance Test Development. Electronic Instrumentation. Textile and Apparel Technology Center. Technical Analysis. Oifice of Weights and Measures. Office of Engineering Standards. Office of Invention and Innovation. Office of Technical Resources. Clearinghouse for Federal Scientific and Technical Information.**

Central Radio Propagation Laboratory.* Ionospheric Telecommunications. Tropospheric Telecommunications. Space Environment Forecasting. Aeronomy.

\footnotetext{
* Located at Boulder, Colorado 80301.

** Located at 5285 Port Royal Road, Springfield, Virginia 22171.
} 


\title{
NATIONAL BUREAU OF STANDARDS Eechnical Mote 18-22 \\ Issued August 2, 1965
}

\author{
QUARTERLY RADIO NOISE DATA \\ MARCH, APRIL, MAY, 1964 \\ W. Q. Crichlow, R. T. Disney, and M. A. Jenkins \\ Central Radio Propagation Laboratory \\ National Bureau of Standards \\ Boulder, Colorado
}

NBS Technical Notes are designed to supplement the Bureau's regular publications program. They provide a means for making available scientific data that are of transient or limited interest. Technical Notes may be listed or referred to in the open literature. 



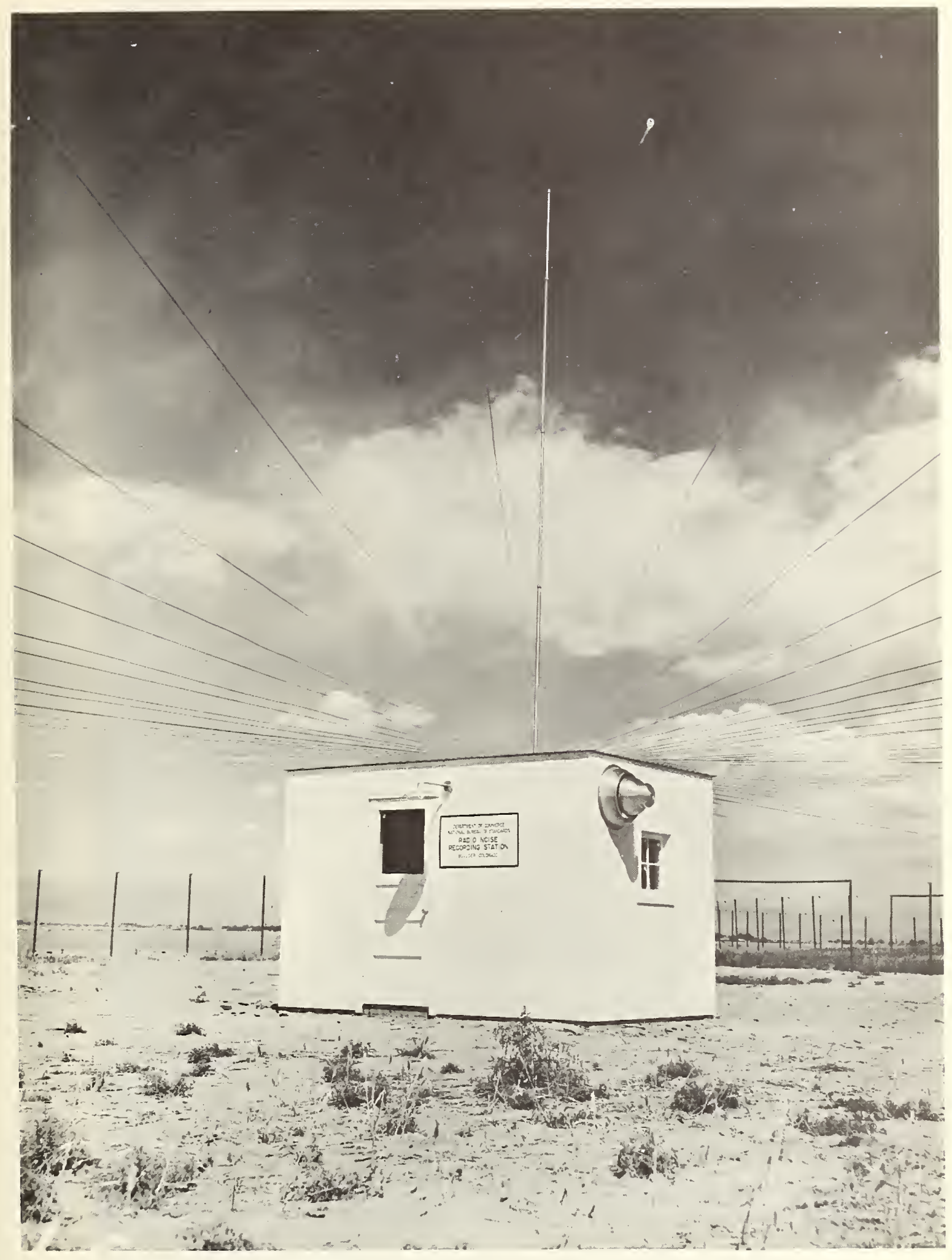

Radio Noise Recording Station 


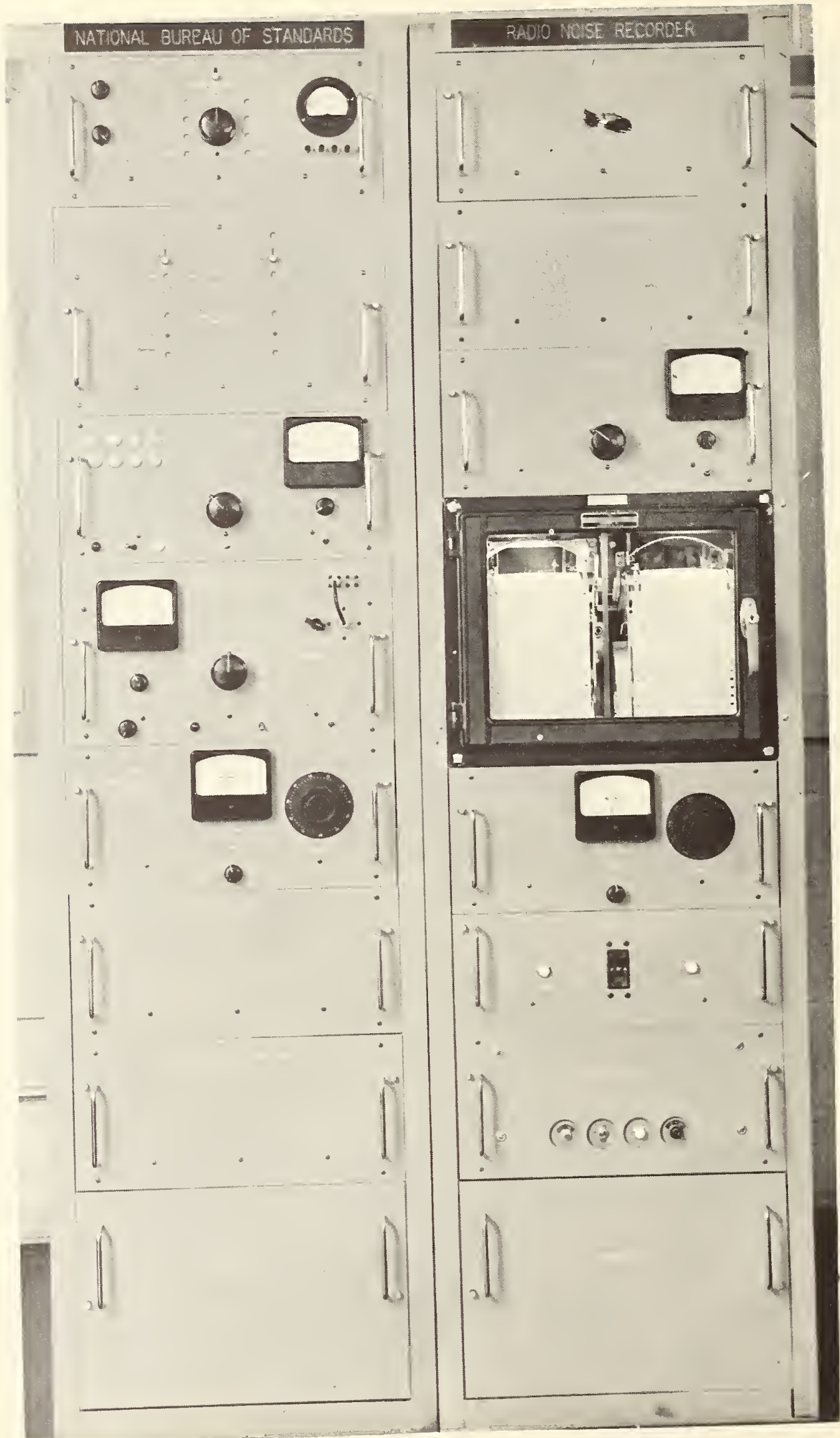

ARN-2 Atmospheric Radio Noise Recorder 


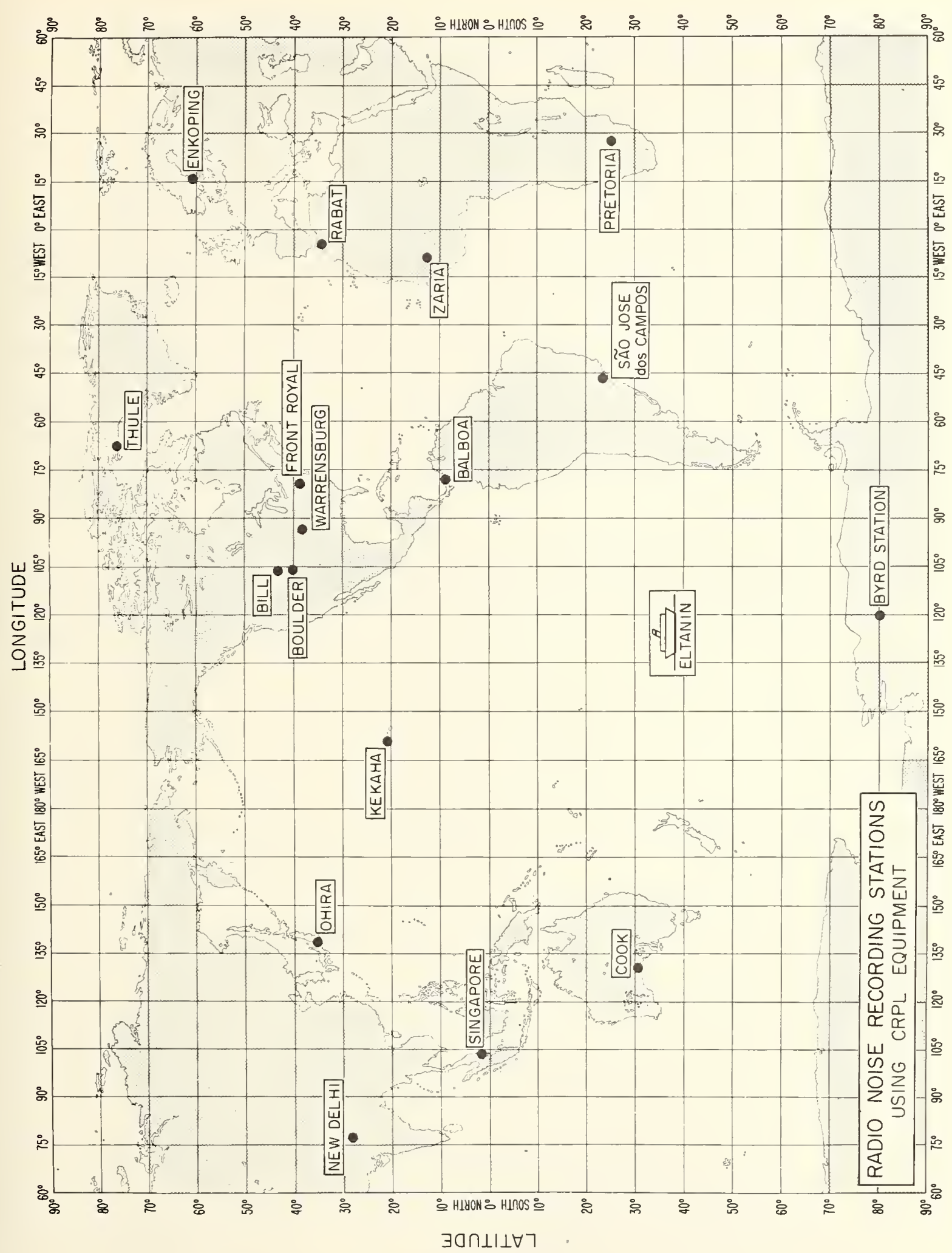




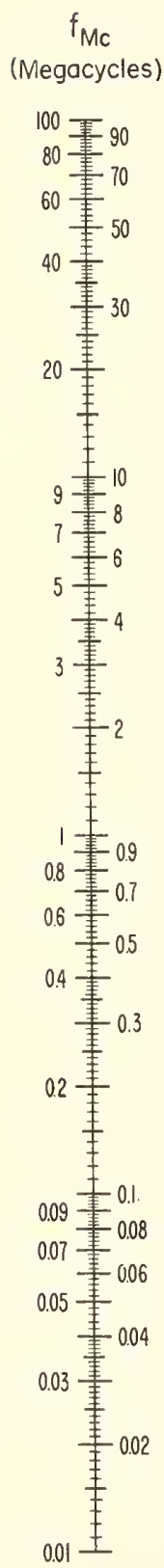

$F_{a}$

(db above $k+b)$
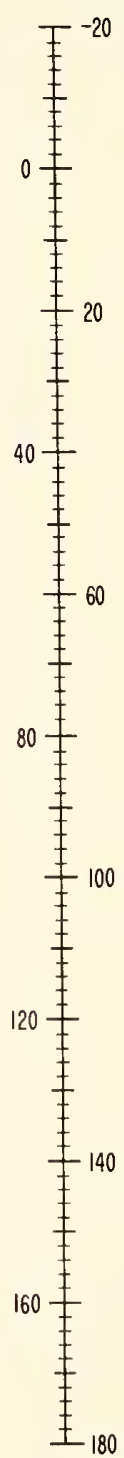

$E_{n}$

( $\mathrm{db}$ above $\mathrm{I} \mu \mathrm{v} /$ meter)

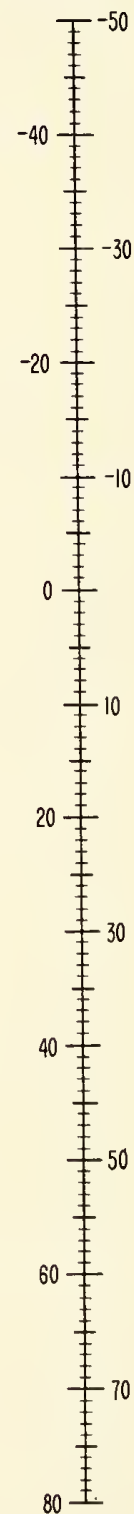

$$
E_{n}=F_{a}+20 \log _{10} f_{M c}-65.5
$$

$F_{a}=$ Effective Antenna Noise Figure = External Noise Power Available from an Equivalent Short, Lossless, Vertical Antenna in db Above ktb. $E_{n}=$ Equivalent Vertically Polarized Ground Wave R.M.S. Noise Field Strength in $\mathrm{db}$ Above I $\mu \mathrm{v} /$ meter for a I kc Bandwidth. $f_{M C}=$ Frequency in Megacycles. 
W. Q. Crichlow, R. T. Disney, and M. A. Jenkins

Radio noise measurements are being made at eighteen stations in a world-wide network operated in a co-operative program co-ordinated by the National Bureau of Standards. The locations of these stations are shown on the map. The results of these measurements for the months of March, April, and May are given in this report. Where the results for the se months are not presently available, the data will be published in subsequent reports, and the data for previous months, which are now available but have not been published previously, are included. The tabulated values are based on three basic parameters of the noise; these are the mean power, the mean envelope voltage, and the mean logarithm of the envelope voltage.

The noise power received from sources external to the antenna averaged over a period of several minutes is the basic parameter and can be conveniently expressed in terms of an effective antenna noise factor, $f_{a}$, which is defined by:

$$
f_{a}=p_{n} / k T_{0} b=T_{a} / T_{0}
$$

where

$$
\begin{aligned}
\mathrm{P}_{\mathrm{n}}= & \text { noise power available from an equivalent loss }-\mathrm{free} \\
& \text { antenna (watts) } \\
\mathrm{k}= & \text { Boltzman's constant }=1.38 \times 10^{-23} \text { joules per degree } \\
& \text { Kelvin } \\
\mathrm{T}_{\mathrm{o}}= & \text { reference temperature, taken as } 288^{\circ} \mathrm{K} \\
\mathrm{b}= & \text { effective receiver noise bandwidth (c/s) } \\
\mathrm{T}_{\mathrm{a}}= & \text { effective antenna temperature in the presence of external } \\
& \text { noise. }
\end{aligned}
$$


The antenna noise factors in this report are for a short vertical antenna over a perfectly conducting ground plane and are expressed in decibels, $F_{a}\left(=10 \log _{10} f_{a}\right)$. This parameter is simply related to the rms noise field strength along the antenna by:

$$
E_{n}=F_{a}-95.5+10 \log _{10} b+20 \log _{10} f M c / s
$$

where:

$$
\begin{aligned}
\mathrm{E}_{\mathrm{n}}= & \mathrm{rms} \text { noise field strength for bandwidth } \mathrm{b} \text { in } \mathrm{db} \text { above } \\
& 1 \mu \mathrm{V} / \mathrm{m} \\
\mathrm{b} \quad= & \text { effective receiver noise bandwidth in } \mathrm{c} / \mathrm{s} \\
\mathrm{f}_{\mathrm{Mc} / \mathrm{s}}= & \text { frequency in } \mathrm{Mc} / \mathrm{s} .
\end{aligned}
$$

The value of $E_{n}$ for a $1 \mathrm{kc} / \mathrm{s}$ bandwidth can be found from the attached nomogram. It should be noted that $E_{n}$ is the vertical component of the field at the antenna. It should also be noted that the rms envelope voltage is $3 \mathrm{db}$ higher than the rms voltage.

The other two noise parameters tabulated are given relative to the mean power. Thus, the mean voltage and mean logarithm expressed as deviations, $V_{d}$ and $L_{d}$, respectively, are in db below the mean power.

Measurements of the three parameters reported were made with the National Bureau of Standards' Radio Noise Recorder, Model ARN-2, which has an effective noise bandwidth of about $200 \mathrm{c} / \mathrm{s}$ and uses a standard 6.6294 meter (21.75') vertical antenna. A fifteen-minute recording is made on each of eight frequencies two at a time during each hour, and these fifteen-minute samples are taken as representing the noise conditions for the full hour during which they were recorded. The month-hour medians, $F_{\text {am }}, V_{\mathrm{dm}}$ and $\mathrm{L}_{\mathrm{dm}}$ are determined from the se hourly values for each of the corresponding parameters. Normally from twenty-five to thirty observations of the mean power are obtained monthly for each hour of the day and from ten to fifteen observations of the voltage and logarithm deviations. When there are fewer than fifteen observations of the mean power or seven observations of the voltage and logarithm deviations, the tabulated values are identified by an asterisk. 
The upper and lower decile values of $F_{a}$ are also reported in the following tabulation to give an indication of the extent of the variation of the noise power from day to day at a given time of day. These are expressed in $\mathrm{db}$ above and below the month-hour median, $\mathrm{F}_{\mathrm{am}}$, and designated by $D_{u}$ and $D_{\ell}$, respectively.

In addition to these month-hour values, corresponding values are tabulated for the time blocks as defined by CCIR Report 322. All recorded values for the four hours of the day and the three-month period are used to determine the median and decile values. When no data were available for one or two months of the season, it is so indicated and should be noted when considering seasonal trends.

The values presented in the tables reflect the actual measured values of radio noise. The only editing for man-made noise or station contamination of the records has been done by the station operators, and no additional attempt has been made to identify these values by systematic statistical means. These preliminary data values are presented in order to expedite dissemination of the data, and additional analyses, in which an attempt is made to eliminate contaminated data, are presented in other publications. The parameter that will first reflect any such contamination will be the logarithmic parameter, $L_{d}$. This contamination generally will cause the value of $L_{d}$ to be less than it would have been had the recorded value been only atmospheric noise. In determining the amplitude-probability distribution from the three measured moments [Crichlow et al., 1960b] contaminated values of $L_{d}$ may be found that will not give a solution of the amplitude-probability distribution. When this occurs, it is suggested that the measured value of $L_{d}$ be ignored and the most probable value of $L_{d}$ from the curve on the graph of $L_{d}$ vs. $V_{d}$ be used. The most probable value has been determined as the best fit for the integrated moments from over sixty measured amplitudeprobability distributions of uncontaminated atmospheric radio noise. The second curve on the graph indicates the minimum value of $L_{d}$ that will give an amplitude-probability distribution with a form factor described in the above reference and can, therefore, be used to determine whether the measured value or the most probable value of $L_{d}$ for any value of $V_{d}$ should be used.

Station clocks are set to local standard time (LST) which is taken from the time zone in which the station is located and is always an integral number of hours different than universal or Greenwich time (see table on page 5). The data from the Floating Antarctic Research Vessel, USNS Eltanin, are grouped so that a block $10^{\circ}$ in latitude by $15^{\circ}$ in longitude is treated as a separate station. The station clock in this case is 
corrected to the LST at the center of the block. Because of this grouping, very few readings may be used to obtain the median values tabulated in some cases. If, during the month, fewer than ten readings are obtained for any one block, the decile values are not given. If data for less than three months are used in the time block summaries, this fact is noted on the summary sheet. Because of the small sample size, some caution should be exercised when using these values.

The assistance of the station operators and other personnel of the operating agencies in obtaining the data contained in this report is gratefully acknowledged. Stations in the recording network were operated by the following agencies:

NBS - Bill, Wyoming; Boulder, Colorado; Byrd Station; Front Royal, Virginia; Kekaha, Hawaii; Warrensburg, Missouri; USNS Eltanin

U.S. Army Strategic Communications Command - Balboa, C.Z.; Thule, Greenland

Postmaster General's Department (Australia) - Cook

Board of Telecommunications (Sweden) - Enköping

DSIR (Great Britain) and Ahmadu Bello University, Electrical Engineering Department, Zaria, Northern Nigeria

Ministry of Communications, Wireless Planning and Co-ordination Organization - New Delhi

Radio Research Laboratories (Japan) - Ohira

Telecommunications Research Laboratory (South Africa) - Pretoria Institut Scientifique Cherifien (Morocco) - Rabat

Comissão Nacional des Atividades Espaciais (Brazil) - Saõ José dos Campos

Department of Scientific and Industrial Research (Great Britain) Singapore 
The following publications contain additional information on radio noise:

Clark, C., "Atmospheric Radio-Noise Studies Based on AmplitudeProbability Measurements at Slough, England, during the International Geophysical Year," Proc. Inst. Elec. Engs., Pt. B, 109, 47, 393 (September, 1962).

Crichlow, W. Q., A. D. Spaulding, C. J. Roubique, and R. T. Disney, "Amplitude-Probability Distributions for Atmospheric Radio Noise," NBS Monograph 23 (November, 1960b).

Crichlow, W. Q., C. J. Roubique, A. D. Spaulding, and W. M. Beery, (January-February, 1960) "Determination of the Amplitude-Probability Distribution of Atmospheric Radio Noise from Statistical Moments," J. Res. NBS 64D (Radio Propagation) No. 1, 49-56.

Crichlow, W. Q., "Noise Investigation at VLF by the National Bureau of Standards," Proc. IRE, 45, 6778 (1957).

Crichlow, W. Q., D. F. Smith, R. N. Morton, and W. R. Corliss, "Worldwide Radio Noise Levels Expected in the Frequency Band 10 Kilocycles to 100 Megacycles," NBS Circular 557, August 25, 1955.

"Report on Revision of Atmospheric Radio Noise Data," C.C.I.R. Report No. 65, VIIIth Plenary Assembly, Warsaw, 1956, (International Radio Consultative Committee, Secretariat, Geneva, Switzerland).

"World Distribution and Characteristics of Atmospheric Radio Noise," C.C.I.R. Report No. 322, Xth Plenary Assembly, Geneva, 1963, (International Radio Consultative Committee, Secretariat, Geneva, Switzerland).

Fulton, F. F. (Jr.) (May-June, 1961), "Effect of Receiver Bandwidth on the Amplitude Distribution of VLF Atmospheric Noise," J. Res. NBS 65D (Radio Propagation) No. 3, 299-304.

Horner, F., "An Investigation of Atmospheric Radio Noise at Very Low Frequencies," Proc. Inst. Elec. Engs., Pt. B, 103, 743 (1956). 
Horner, F., "Radio Noise of Terrestrial Origin," Proc. of Commission IV on Radio Noise of Terrestrial Origin during the XIIIth General Assembly of URSI, " London, September, 1960.

Spaulding, A. D., C. J. Roubique, and W. Q. Crichlow (NovemberDecember, 1962) "Conversion of the Amplitude-Probability Distribution Function for Atmospheric Radio Noise from One Bandwidth to Another," J. Res. NBS 66D (Radio Propagation) No. 6, 713-720.

Obayashi, T. (January-February, 1960), "Measured Frequency Spectra of Very-Low-Frequency Atmospherics," J. Res. NBS 64D (Radio Propagation) No. 1, 41-48.

Taylor, W. L. (September-October, 1963), "Radiation Field Characteristics of Lightning Discharges in the Band $1 \mathrm{kc} / \mathrm{s}$ to $100 \mathrm{kc} / \mathrm{s}$," J. Res. NBS 67D (Radio Propagation) No. 5, 539-550.

Taylor, W. L. and A. G. Jean (September-October, 1959), "VeryLow-Frequency Radiation Spectra of Lightning Discharges," J. Res. NBS 63D (Radio Propagation) No. 2, 199-204.

URSI Special Report No. 7, "The Measurement of Characteristics of Terrestrial Radio Noise," Elsevier Publishing Co. (1962).

Watt, A. D. and E. L. Maxwell, "Characteristics of Atmospheric Noise from 1 to $100 \mathrm{kc}, "$ Proc. IRE, 45, 6, 787 (1957).

Watt, A. D. (September-October, 1960), "ELF Electric Fields from Thunderstorms," J. Res. NBS 64D (Radio Propagation) No. 5, 425-433.

Watt, A. D. and E. L. Maxwell, "Measured Statistical Characteristics of VLF Atmospheric Radio Noise," Proc. IRE, 45, 1, 55 (1957).

Watt, A. D., R. M. Coon, E. L. Maxwell, and R. W. Plush, "Performance of some Radio Systems in the Presence of Thermal and Atmospheric Noise," Proc. IRE, 46, 12, 1914 (1958). 
Data included in this report and the standard time for each station are as follows:

To Convert

LST to GMT

Station

Data

(hours)

$\begin{array}{lllrl}\text { Balboa } & \text { March } & 1964 & 75 \mathrm{~W} & +05 \\ \text { Bill } & \text { March, April, May } & 1964 & 105 \mathrm{~W} & +07 \\ \text { Boulder } & \text { March, April, May } & 1964 & 105 \mathrm{~W} & +07 \\ \text { Cook } & \text { March, April, May } & 1964 & 135 \mathrm{E} & -09 \\ \text { USNS Eltanin } & \text { March, April, May } & 1964 & & \\ \text { Enköping } & \text { March, April, May } & 1964 & 15 \mathrm{E} & -01 \\ \text { Front Royal } & \text { March, April, May } & 1964 & 75 \mathrm{~W} & +05 \\ \text { Kekaha } & \text { March, April, May } & 1964 & 150 \mathrm{~W} & +10 \\ \text { New Delhi } & \text { March, April, May } & 1964 & 75 \mathrm{E} & -05 \\ \text { Ohira } & \text { March, April, May } & 1964 & 135 \mathrm{E} & -09 \\ \text { Pretoria } & \text { March, April, May } & 1964 & 30 \mathrm{E} & -02 \\ \text { Rabat } & \text { March, April, May } & 1964 & \text { GMT } & 0 \\ \text { Sao José } & \text { January, February } & 1964 & 45 \mathrm{~W} & +03 \\ & \text { March, April, May } & & & +06\end{array}$

Previous data from the World-Wide Network have been published in the following Technical Note 18 series:

18 -1 July 1, 1957 -December 31, 1958

18-2 March, April, May, 1959

18-3 June, July, August, 1959

18-4 September, October, November, 1959

18-5 December, January, February, 1959-60

18-6 March, April, May, 1960

18-7 June, July, August, 1960

18-8 September, October, November, 1960

18-9 December, January, February, 1960-61

18-10 March, April, May, 1961

18-11 June, July, August, 1961

18-12 September, October, November, 1961

18-13 December, January, February, 1961-62

18-14 March, April, May, 1962

18-15 June, July, August, 1962

18-16 September, October, November, 1962

18-17 December, January, February, 1962-63 
18 -18 March, April, May, 1963

18-19 June, July, August, 1963

18-20 Sepiember, October, November, 1963

18-21 December, January, February 1963-64 
MOST PROBABLE AND MINIMUM VALUES OF $L_{d}$ VERSUS $V_{d}$ FOR ATMOSPHERIC RADIO NOISE

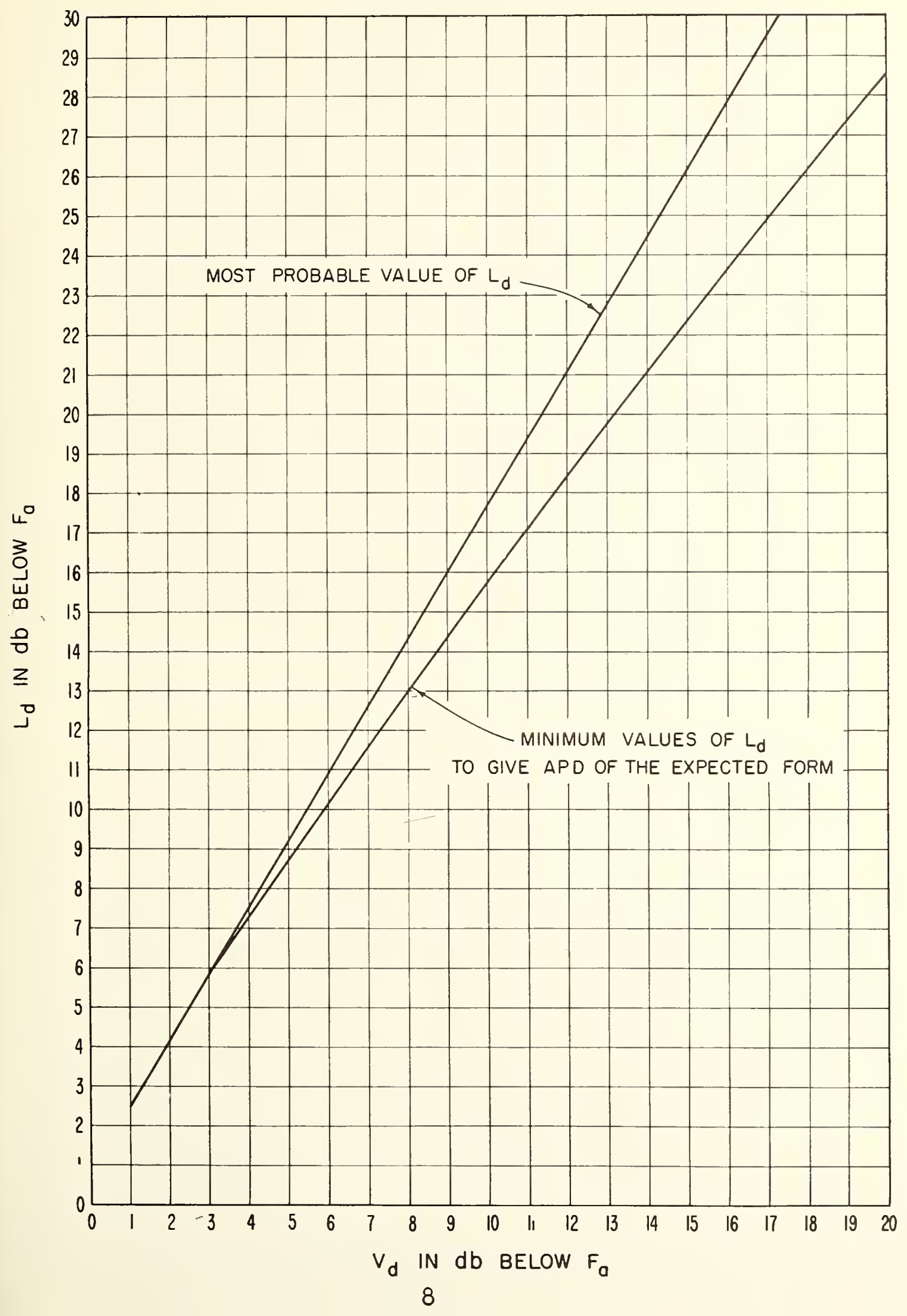


(3)

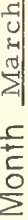

$$
\lambda
$$

$$
\gamma \rightarrow \text { j }>
$$

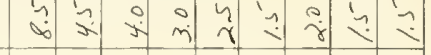

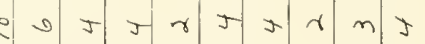
E *

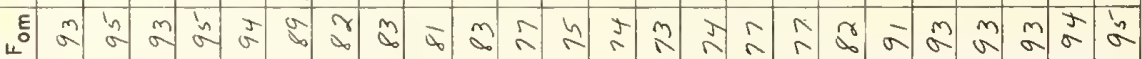

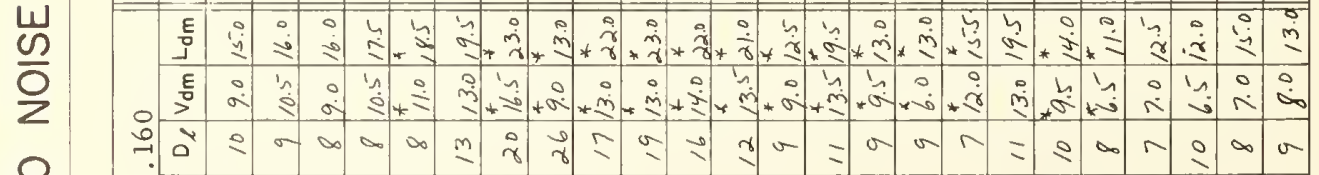

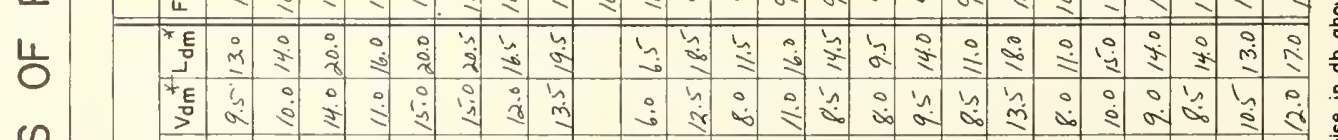

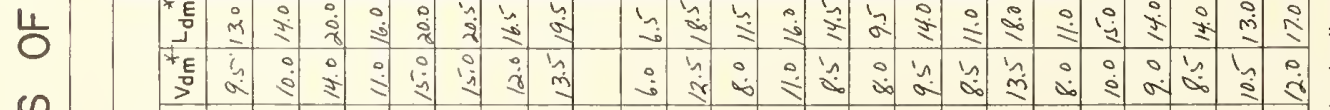

$\stackrel{1}{3}$ เก ธิ

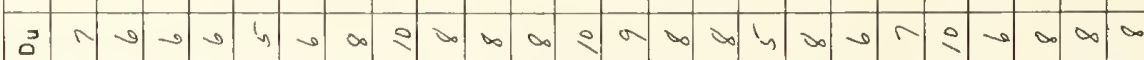

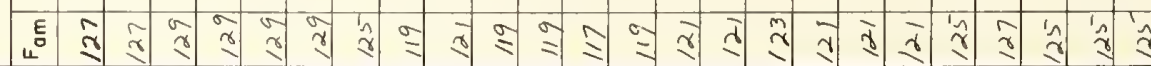

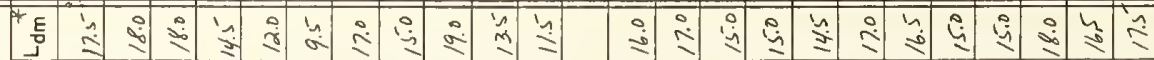

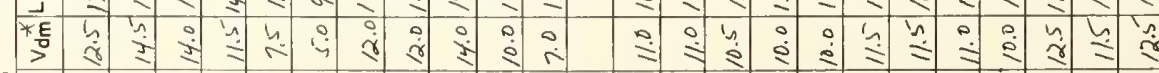

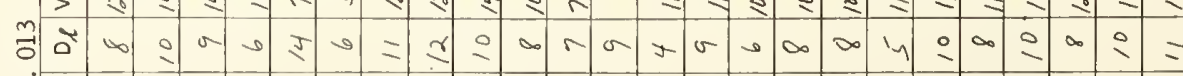

I 5 כ 


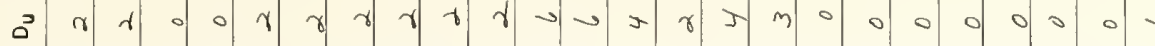

Е

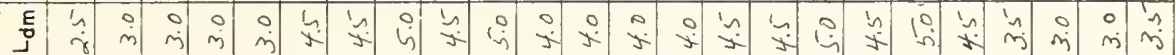

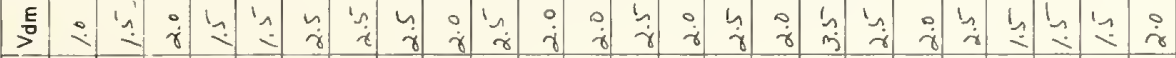

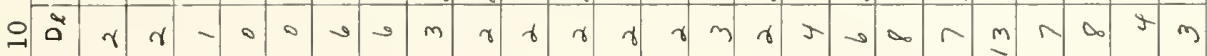

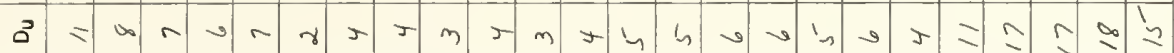

3
ำ

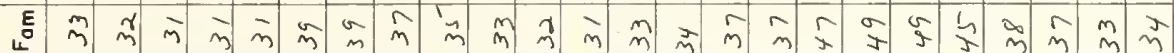

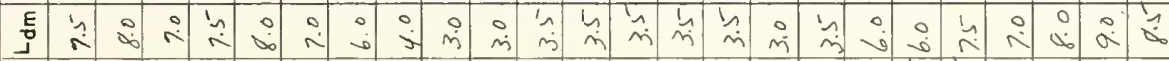

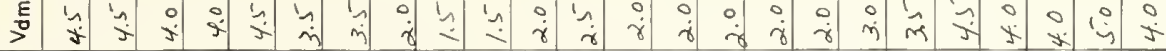

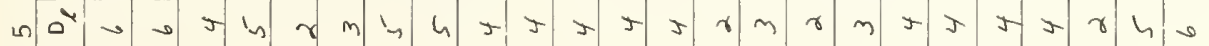

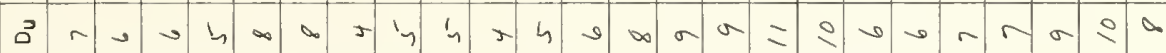

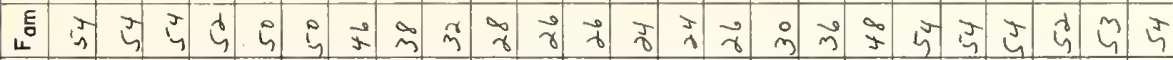

ठ்

j

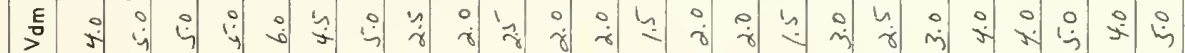

ก

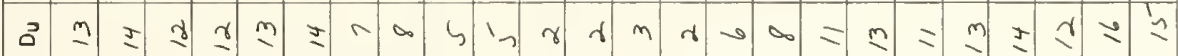

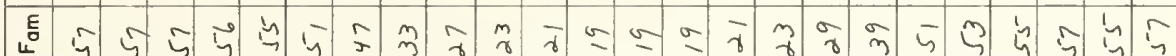

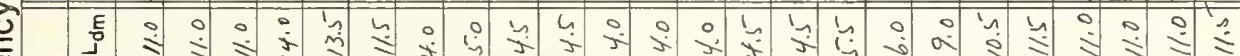

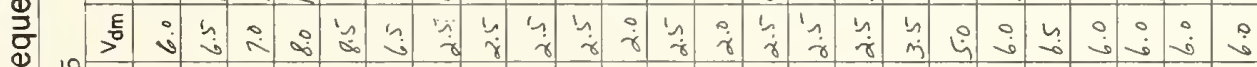
L $\stackrel{2}{4}$

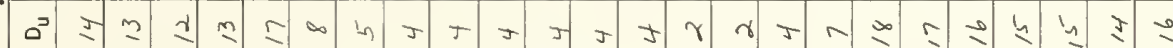

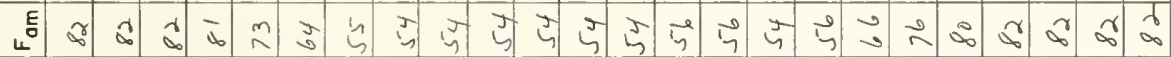

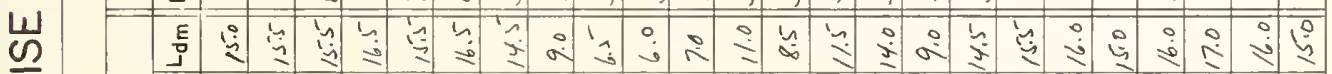

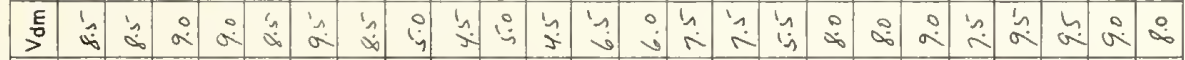
bे

$\frac{0}{8}$

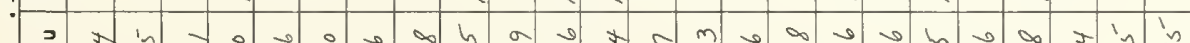

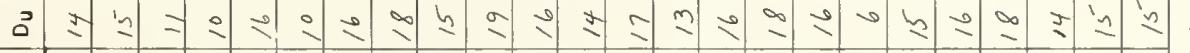

E

重

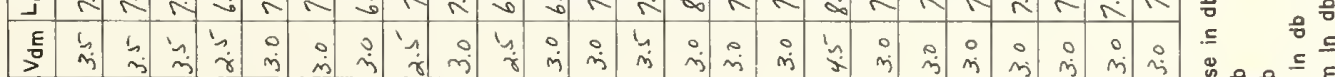

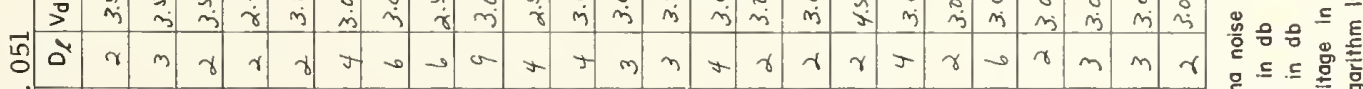

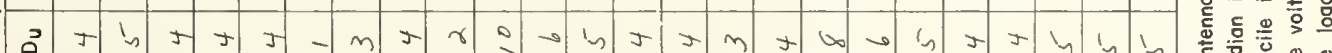

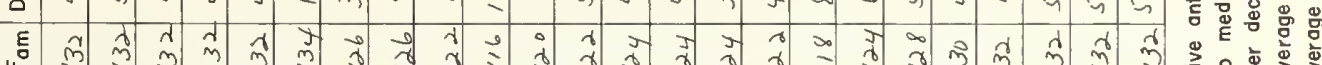
L

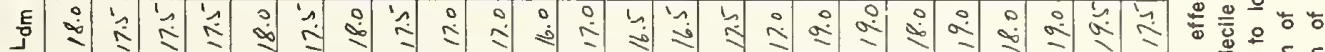

E m)

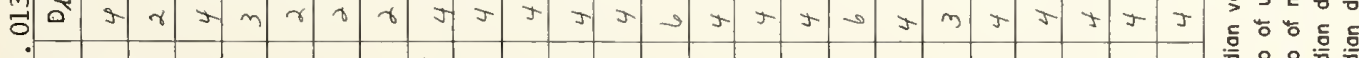

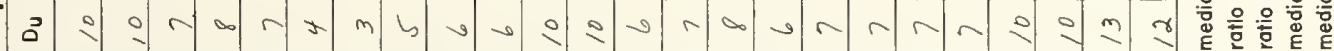

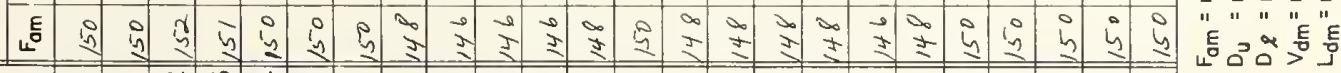
(IS7) גnoH 


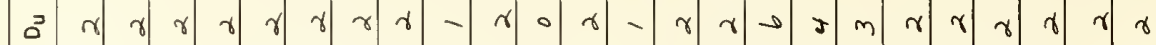

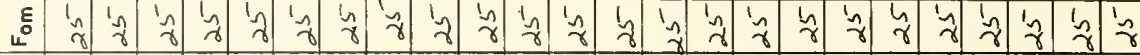

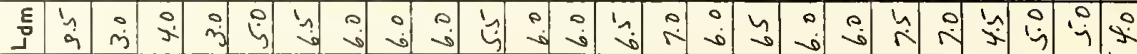

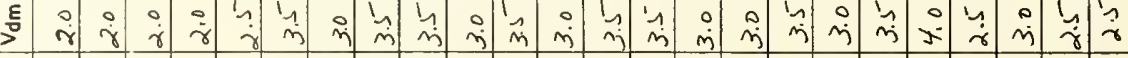
음

龺

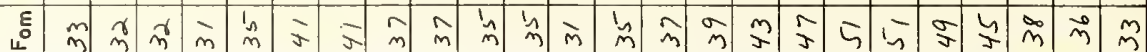

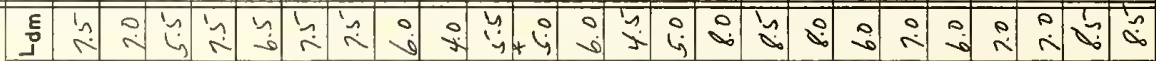
E

in 0

ว: $\infty$ :

है

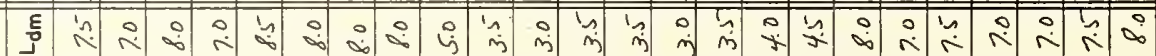

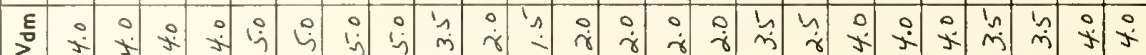

령

ن व ㄱำ

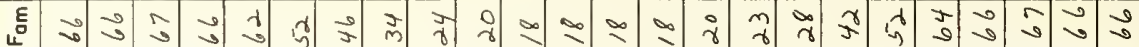

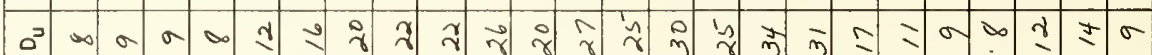

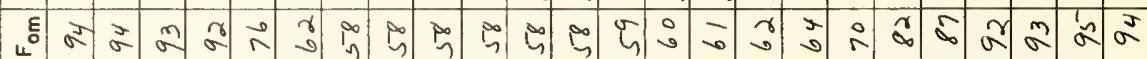

$\frac{1}{0}$
$\frac{1}{2}$
$\frac{0}{2}$
$\frac{1}{\alpha}$

E

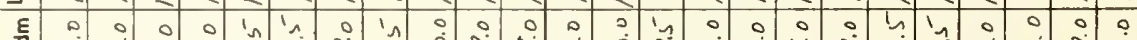

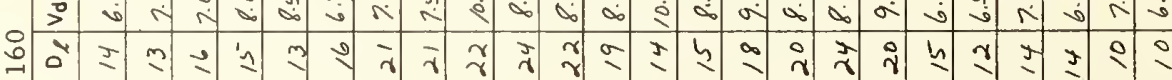

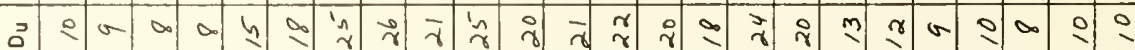

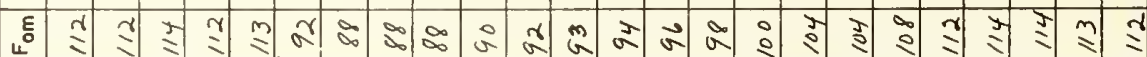

E

แ

E

o

닌

चี ั5

औै

$\frac{1}{5}$

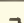

ฉ

E

$\frac{1}{3}$

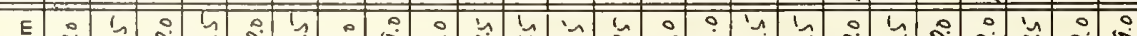

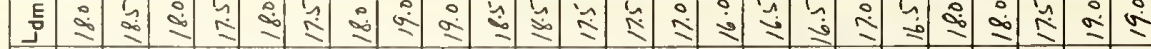

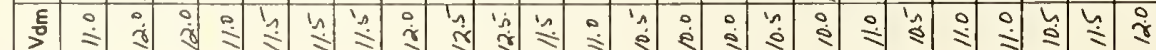
$\stackrel{m}{0}$

I

E 5 क

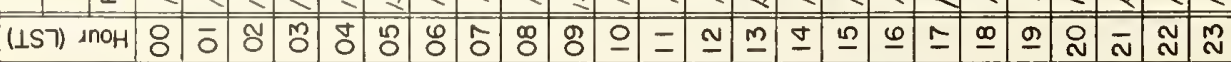


๙

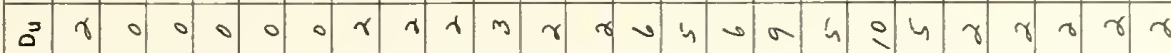

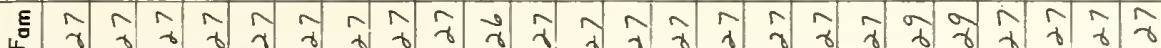
J E

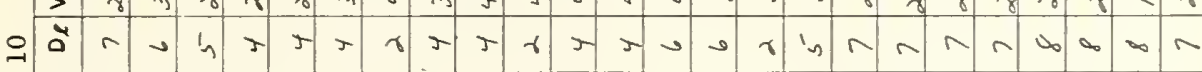

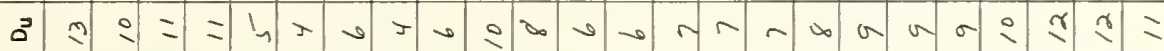
E J

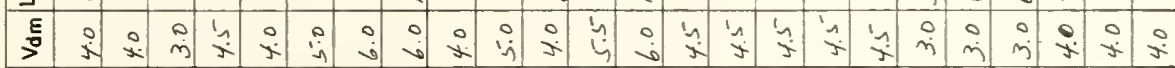

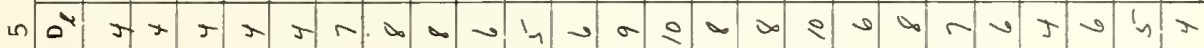

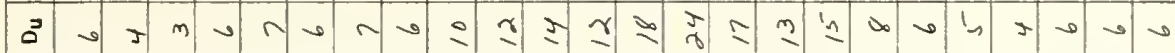

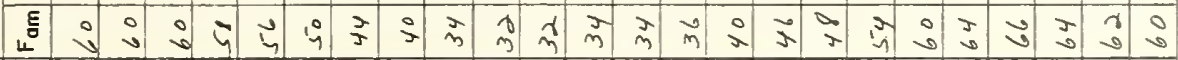
J

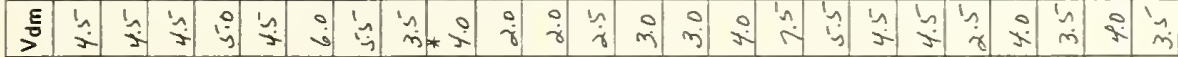

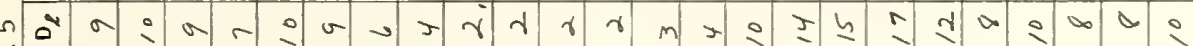

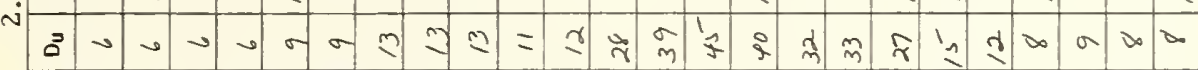

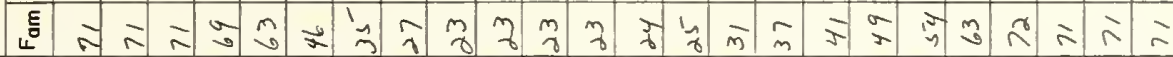

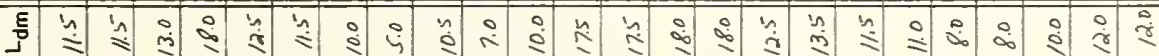

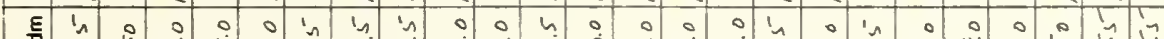

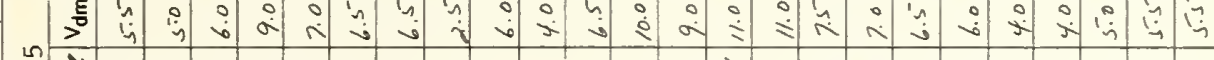

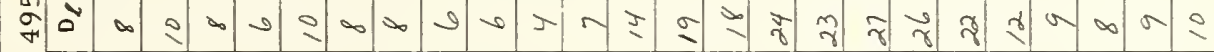

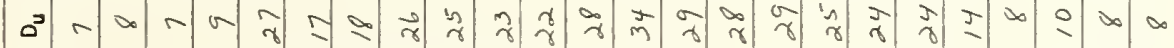

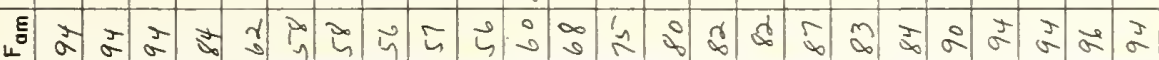

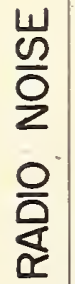

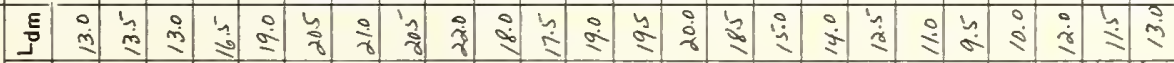

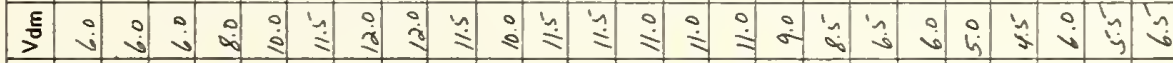

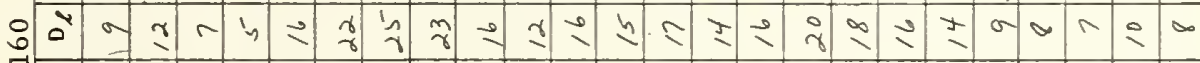

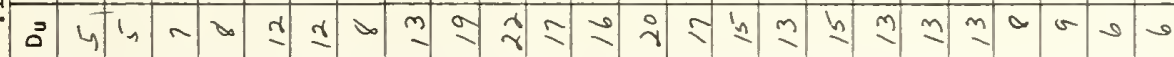

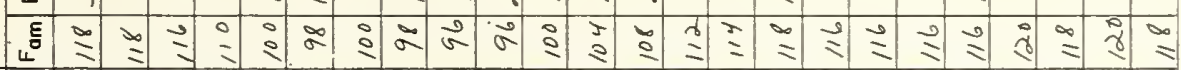

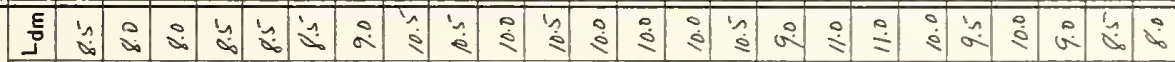

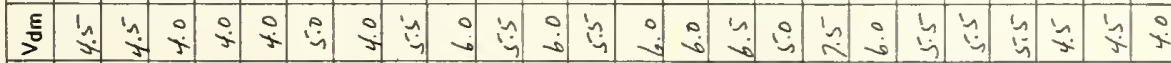

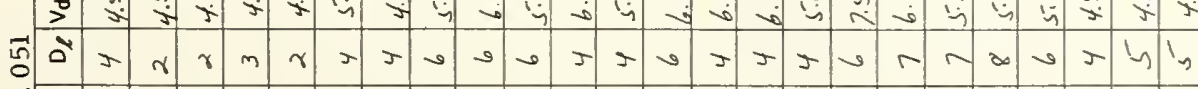

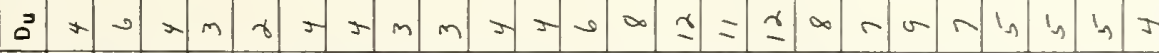

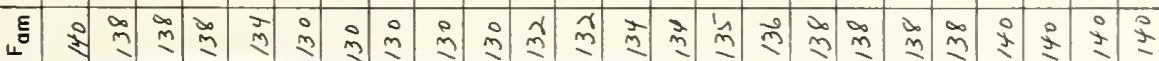

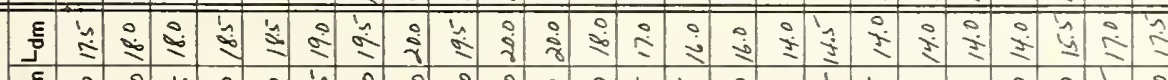

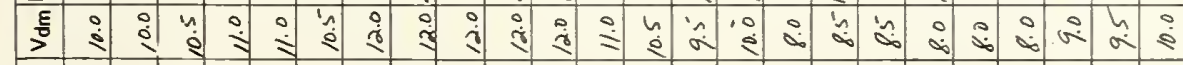

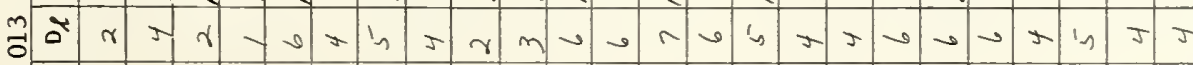

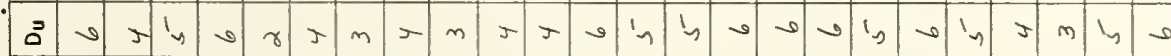

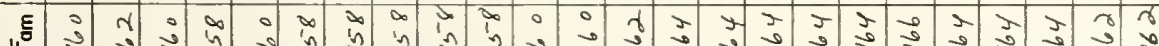

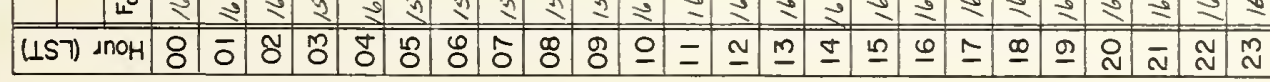




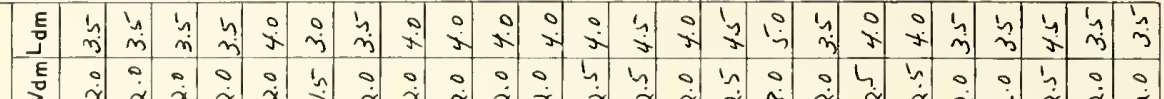

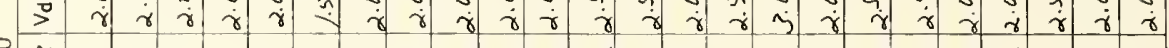

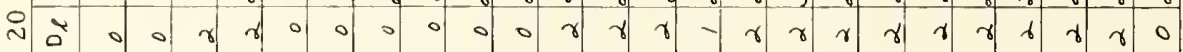

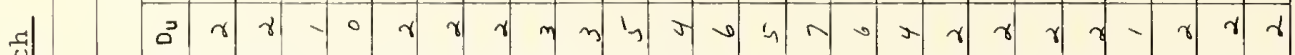

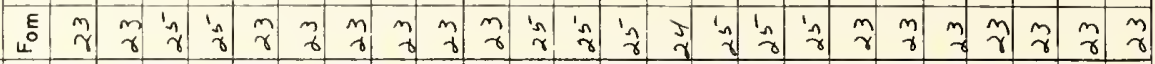

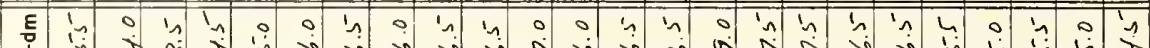

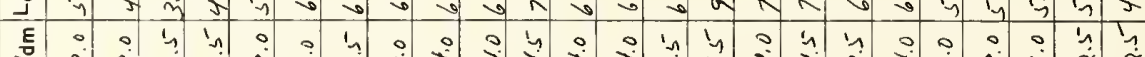

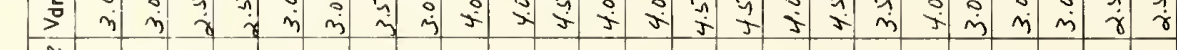

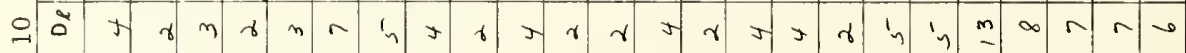

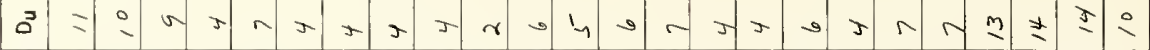

is

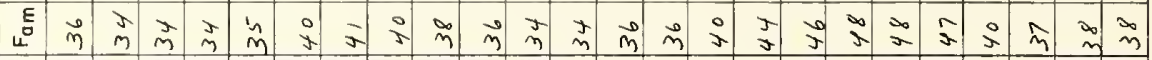

E

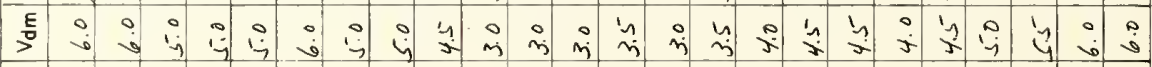

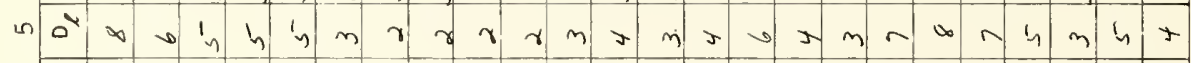

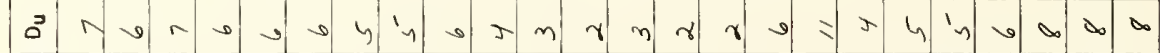

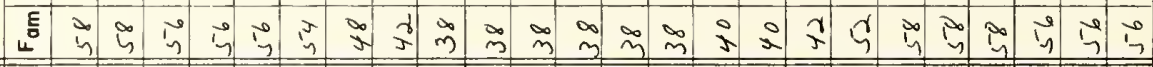

J E

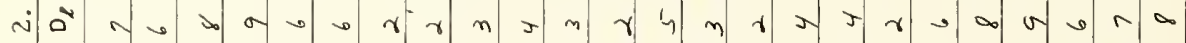

至

厓

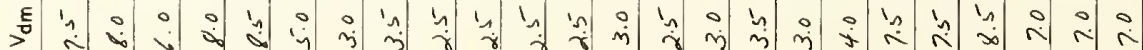

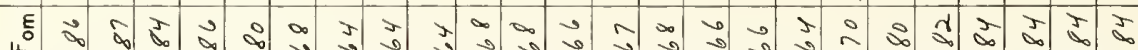

$\frac{1}{2}$
$\frac{0}{2}$
$\frac{0}{0}$
$\frac{1}{\alpha}$

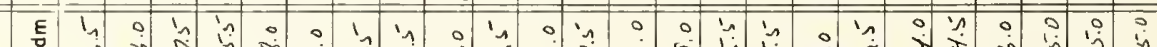

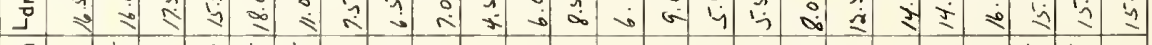

है $\overbrace{0}^{0} a$ a

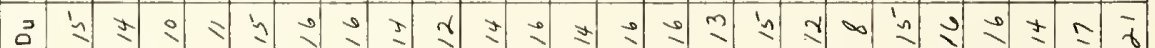

ह ह

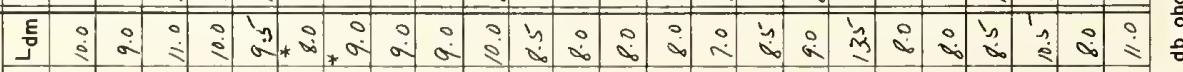

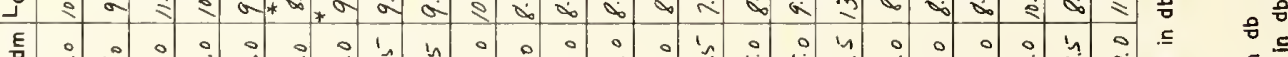

$\stackrel{1}{0}$

崩

>

กึ่

3 $\infty$

E

ह

E

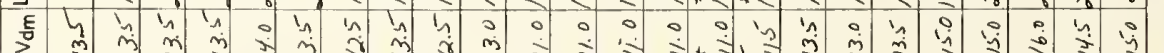

$\stackrel{m}{\overrightarrow{0}}$ 


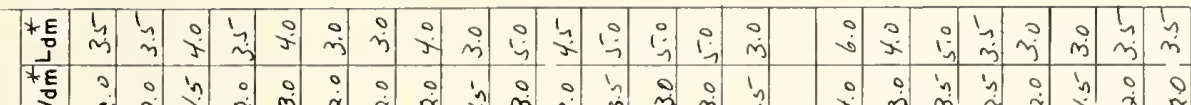

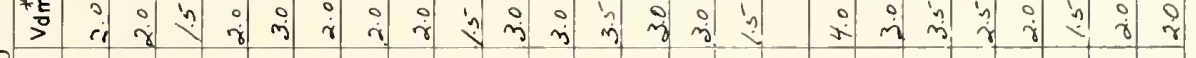

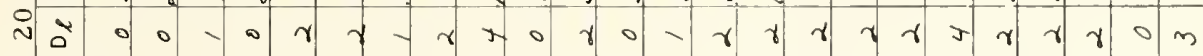

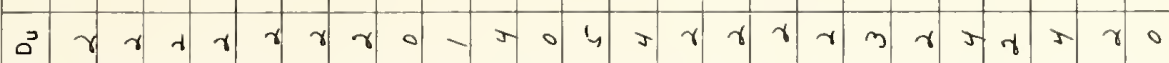
है

*E FE 이 a :

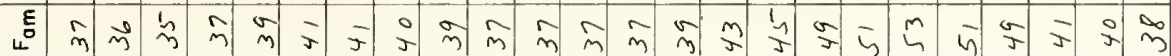
+E *E

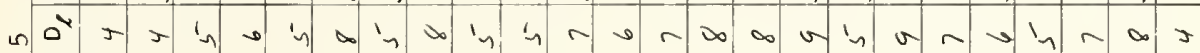

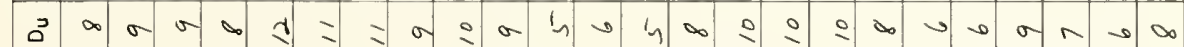

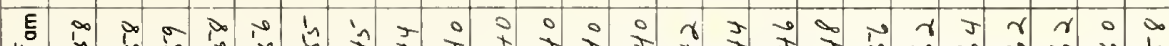

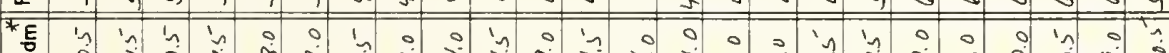
†

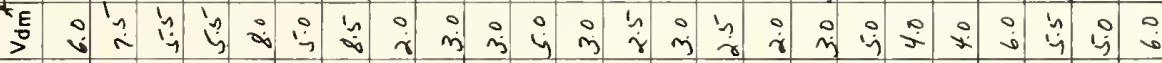

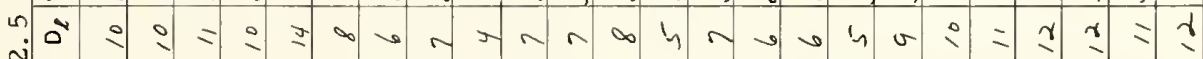

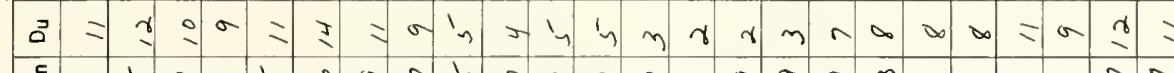
E

当

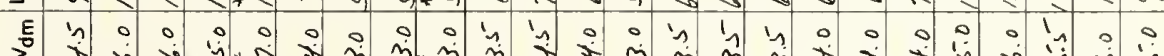
$\frac{\pi}{n}$

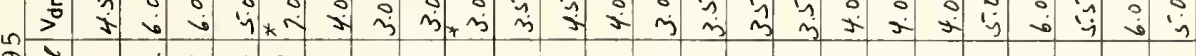

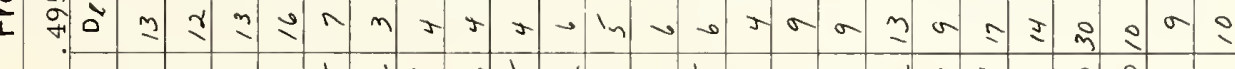

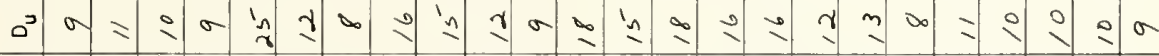
นE 声

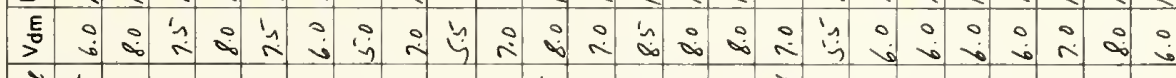

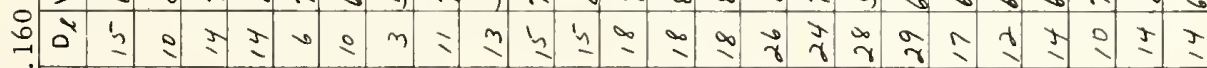
ว แ

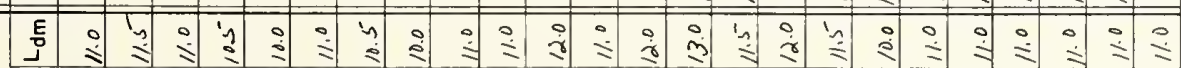
E

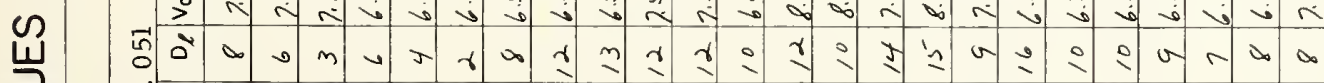

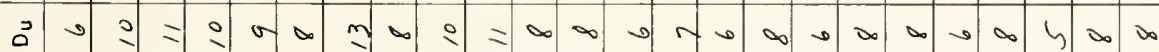
L

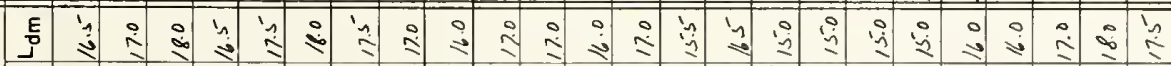
䶮 0
a

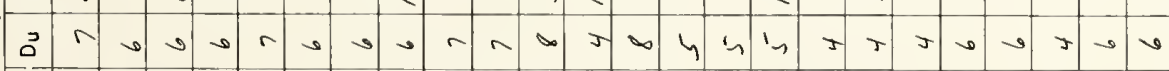

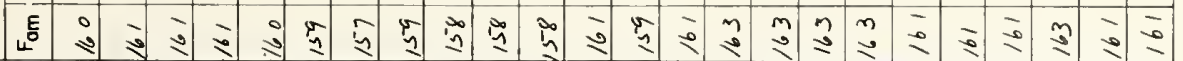

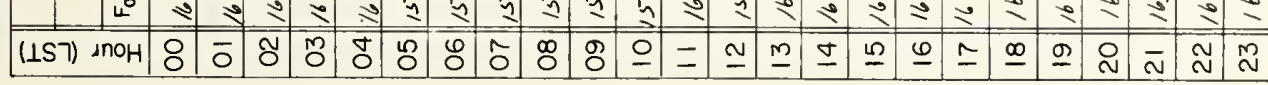




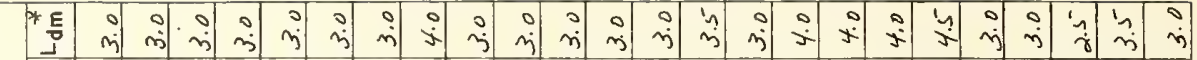
* E

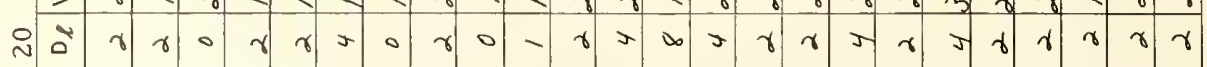
כ

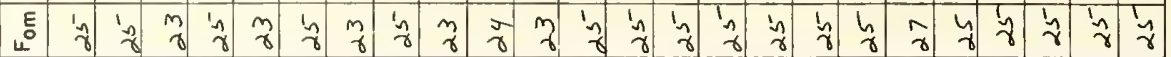

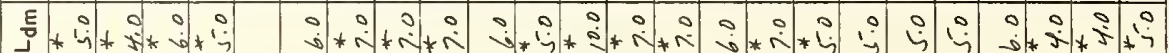
E

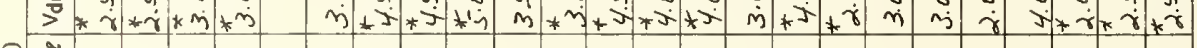
ㅇำ

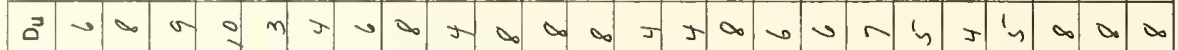

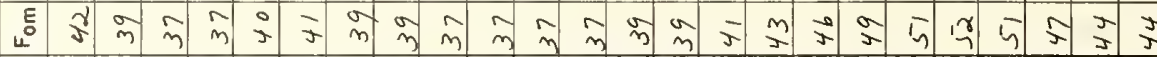

J

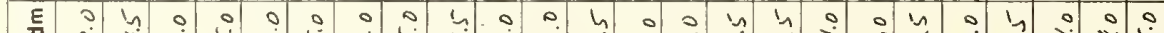

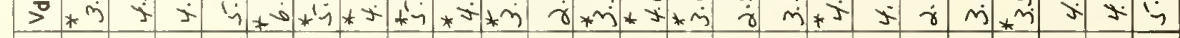
约 $m$ J

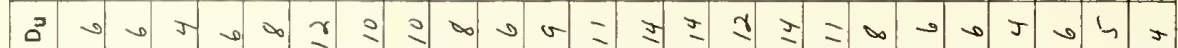

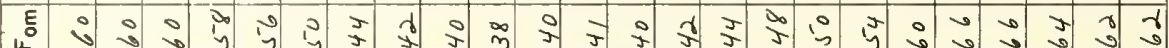

J

E f.

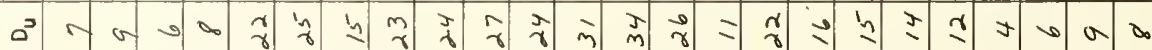

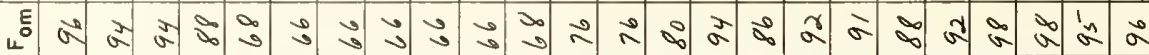

E

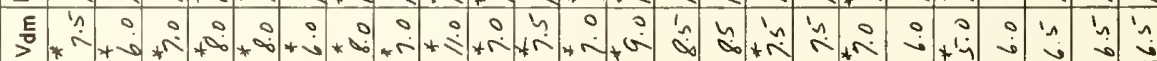

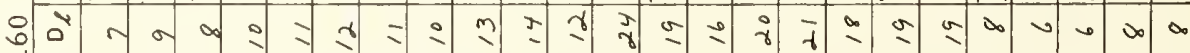

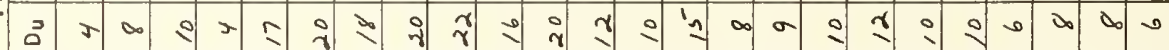
E

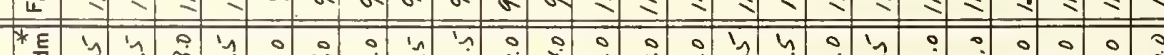

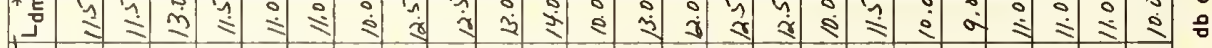

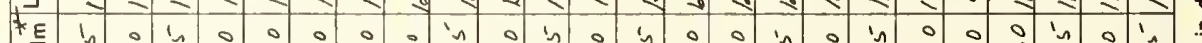

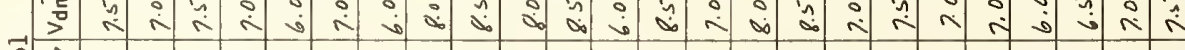
ปี่

3 3.

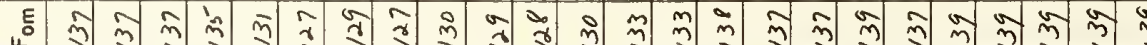

E

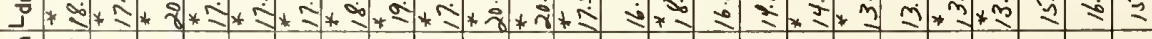

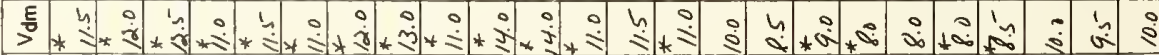

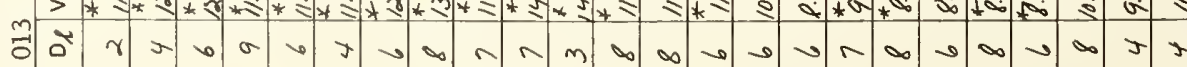

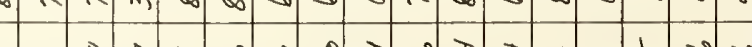

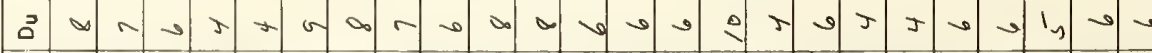


옴

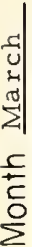

$0^{3}$

$\overrightarrow{0} \rightarrow-0+2 x$

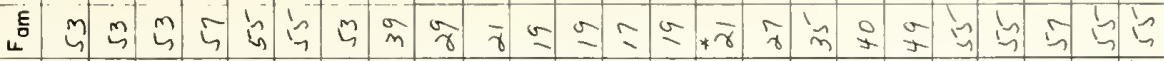

E $\hat{j}$ 守

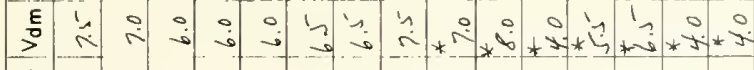

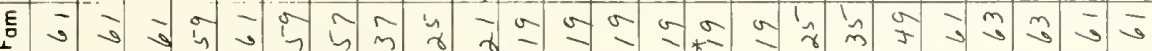

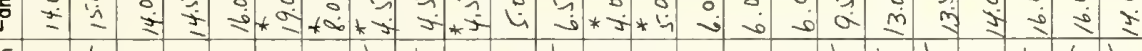

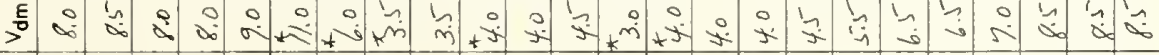

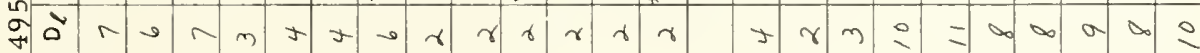

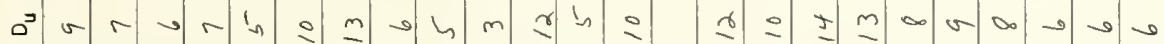

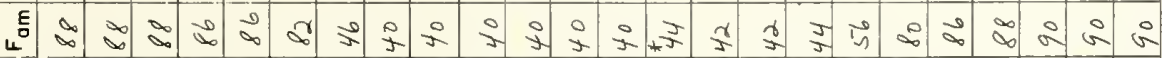

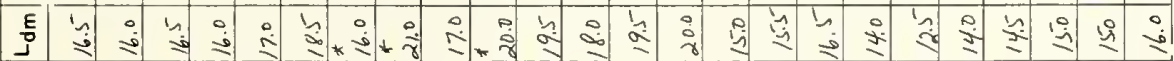

हᄐ

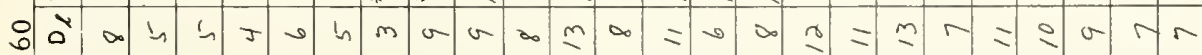

若

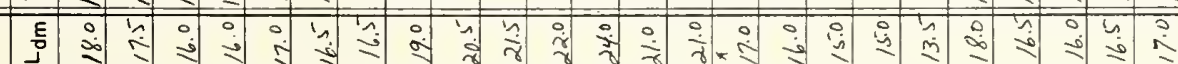

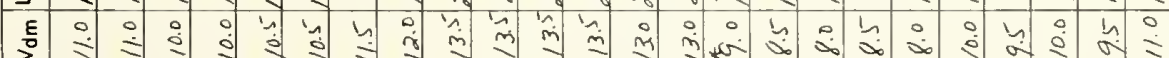

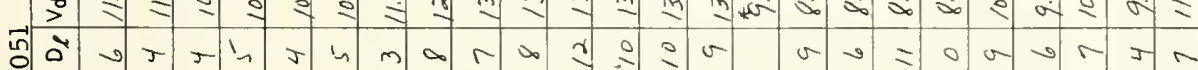

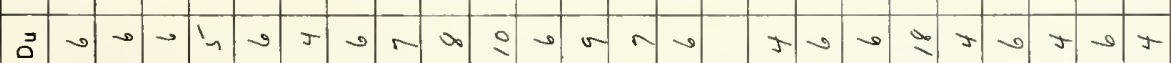

ह

E

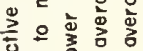




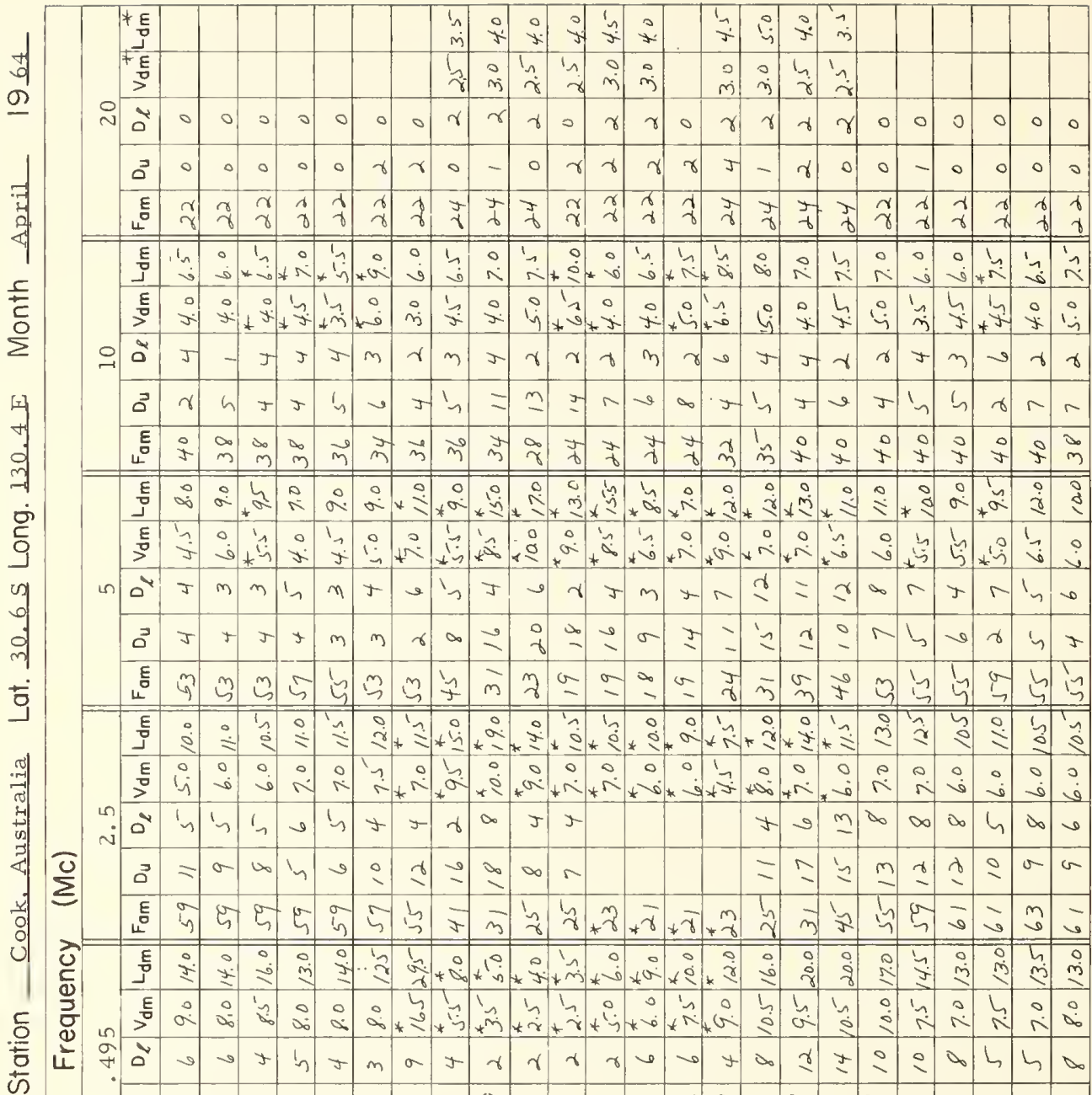

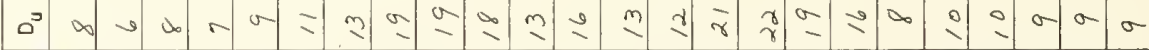
ए

$\frac{w}{2}$

E

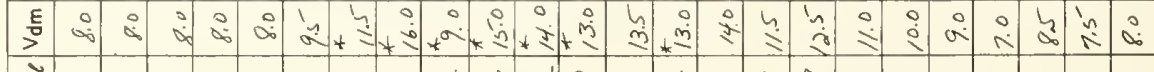

$\frac{0}{2}$ -

ப

E

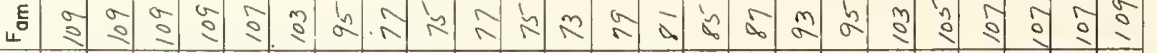

E

E

w

in

(i)

ธ

है के के के ने व

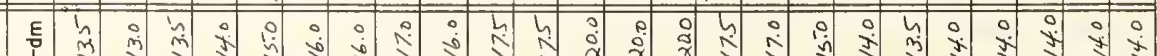

동
$\frac{1}{1}$
$\frac{1}{1}$
$\frac{1}{0}$

E

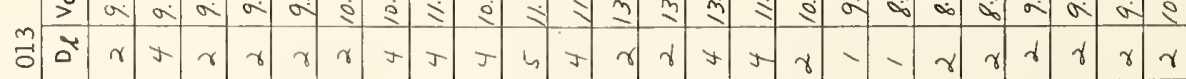

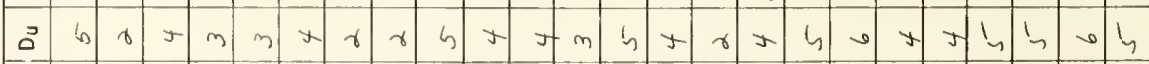

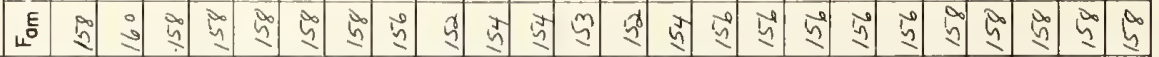

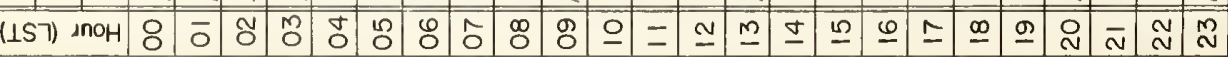




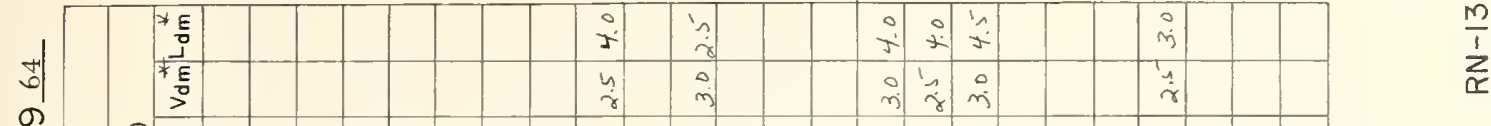

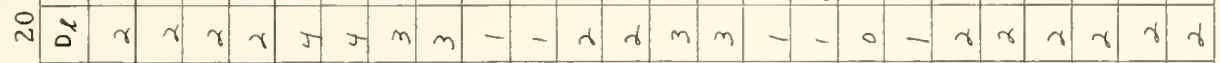

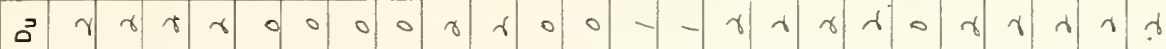

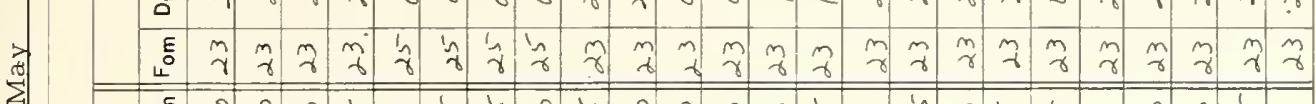

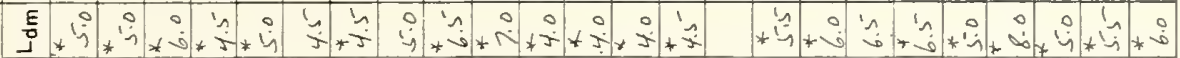

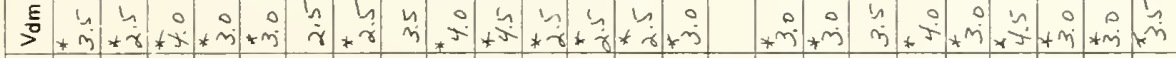
일 $y \gamma \gamma \gamma \gamma \gamma \mathrm{r}$

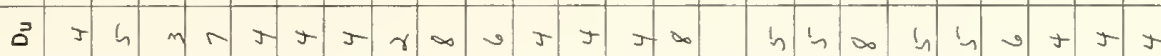

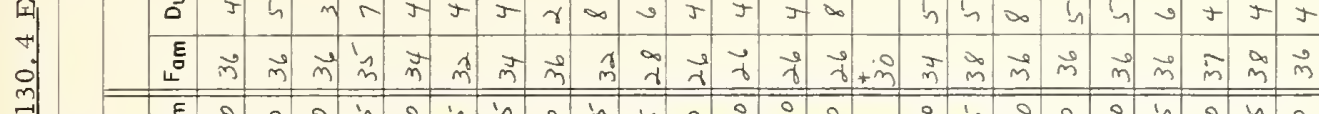

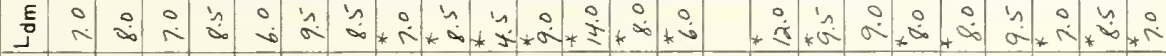

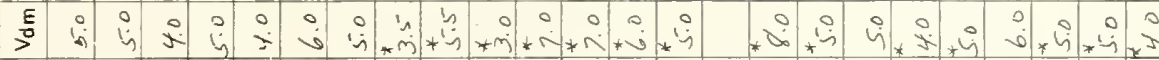
⿰纟勺

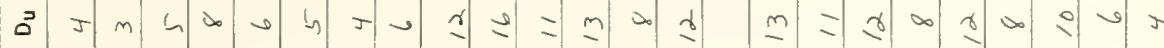
E

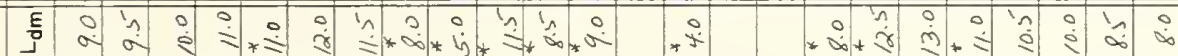

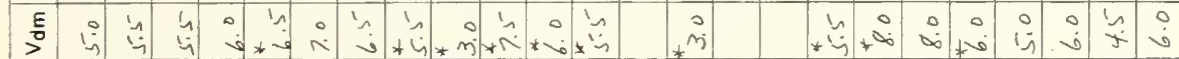
면

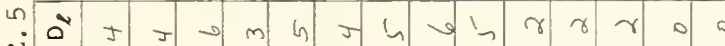
है

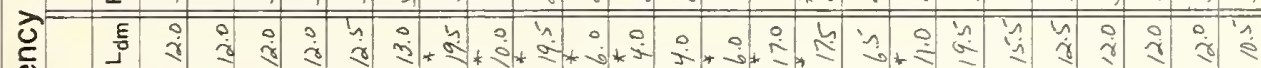

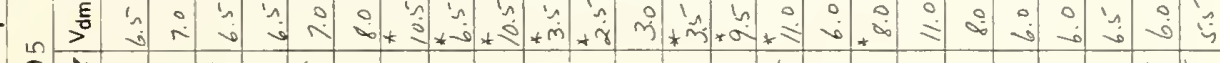

0 น

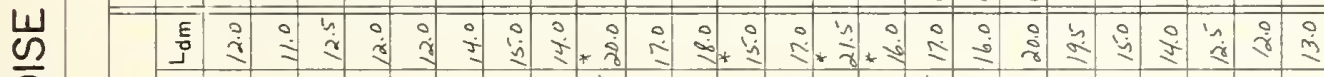

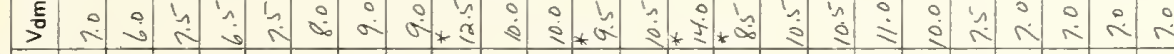
्ְर

$\frac{0}{\square}$

-

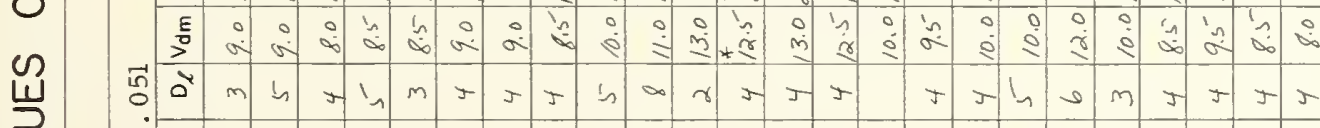
a) ह 先

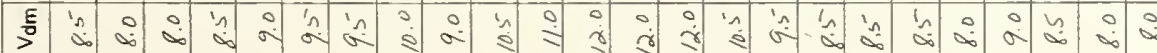
$\stackrel{m}{0}$ ठै 
*E

ह

$>$

요 वे

격

กิำ

ஜ்

*E్

\section{E}

E

官

in $0^{x}$

2

咅 है

$\stackrel{\varepsilon}{>}$

青

in 0

$\sum^{N} 3$

XE :

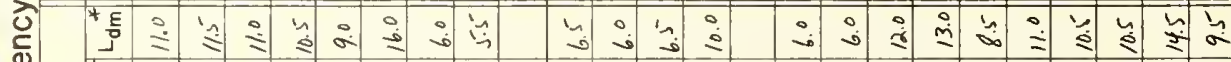

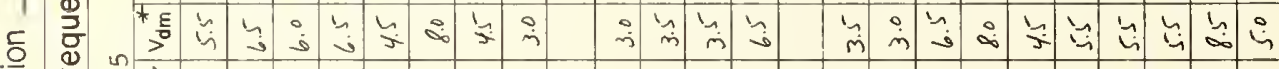

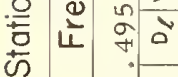

0

ॠ

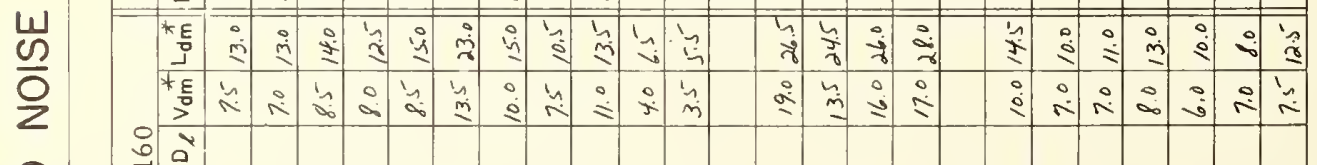

$\frac{0}{\frac{0}{\alpha}}$

3

सE $m$ ह ₹

高言

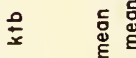

* E

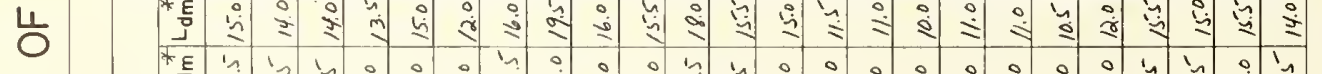

*E

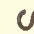

है

แ.

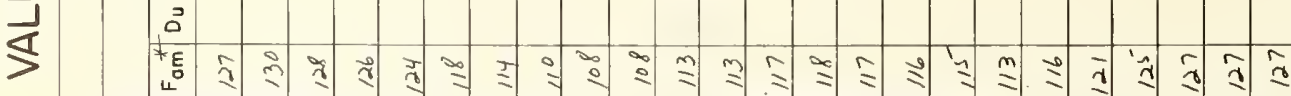

$\frac{0}{3}$

†E

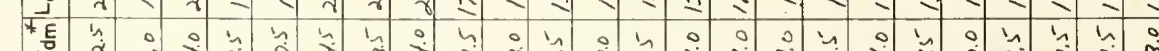
$\stackrel{m}{0}$

$\overrightarrow{0}$ 


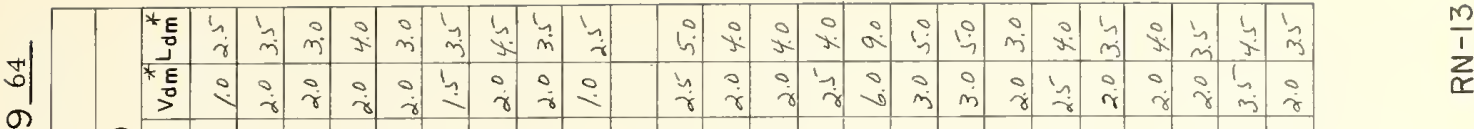

今

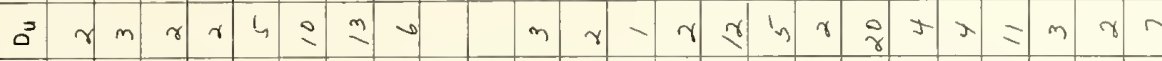

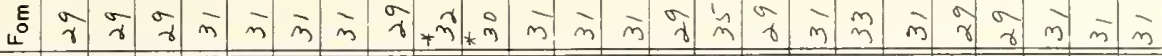

*

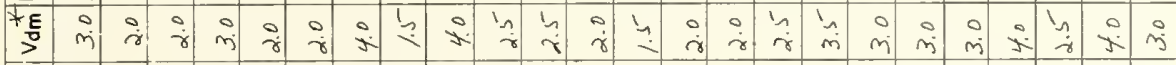

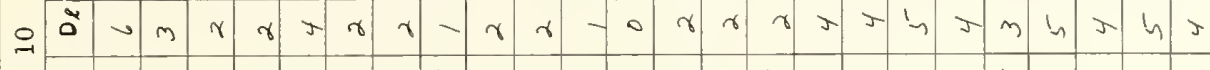
a d o c is

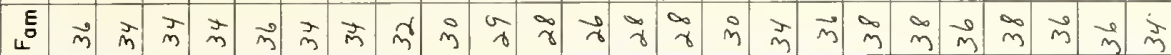
*E

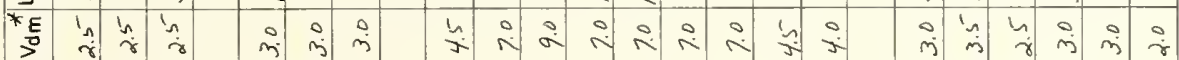

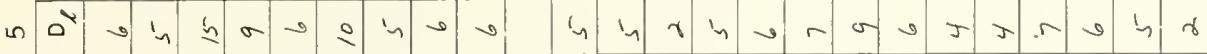

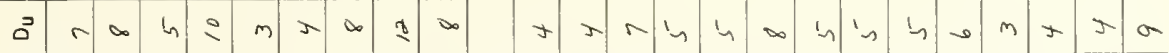
ह *

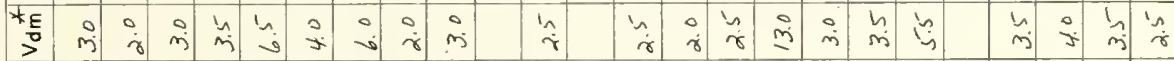
i

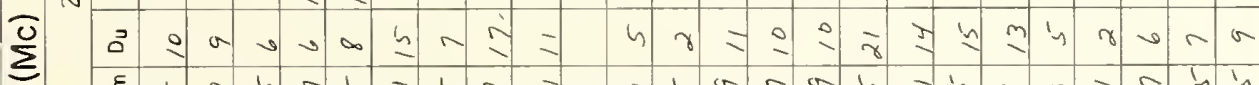

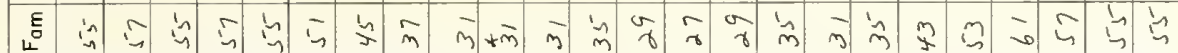
* 음

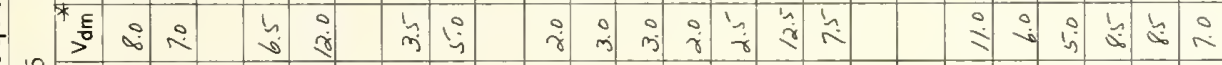

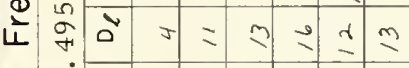

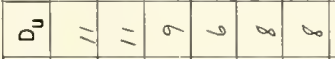

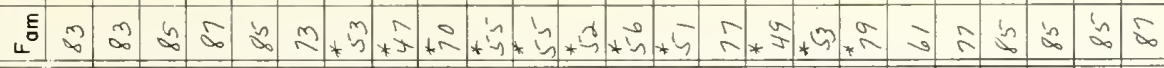

$\frac{w}{2}$

*E

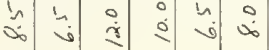

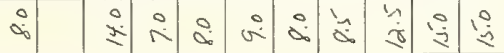

政

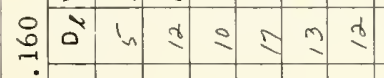

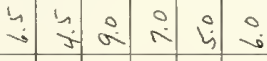
$\stackrel{0}{3}$ aे $* 3$

$\frac{0}{\frac{1}{\alpha}}$ a $₹ a$ or in $\rightarrow$ i

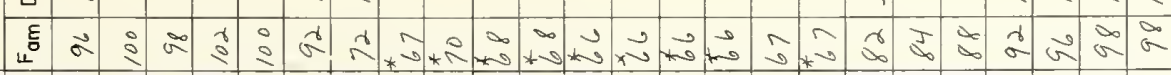
†

* E

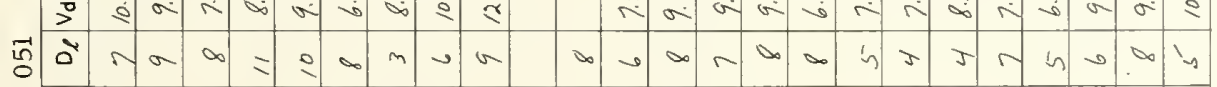
a $\infty$ a

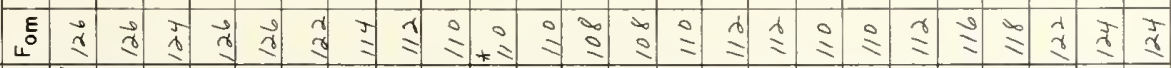

+E

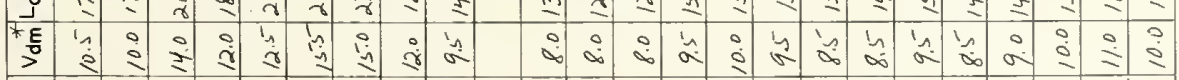
क्व

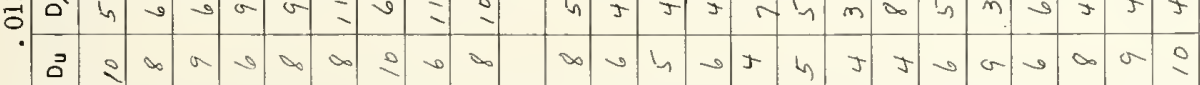

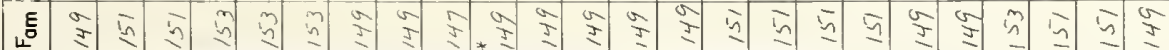

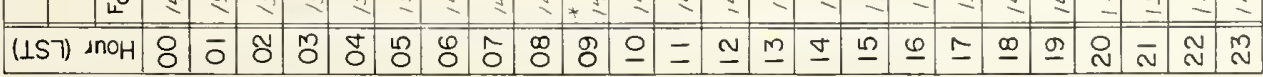




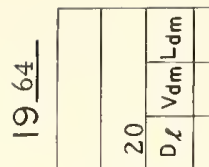

영 ${ }^{3}$

$\stackrel{\sqrt[3]{\mid}}{\mid}$

$\frac{c}{\frac{5}{5}}$

4

E气

통

의 음

3.

ง

Бे

이 क 0

彳ิ

प्ञ

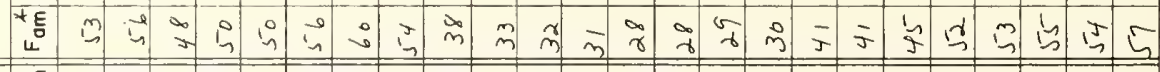

1 .

矛

$\sum^{0}$

in 0

点

E

${ }^{N}$

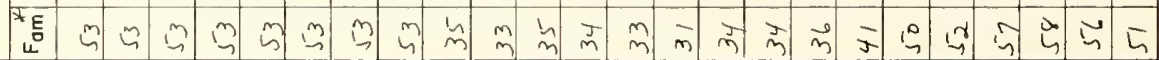

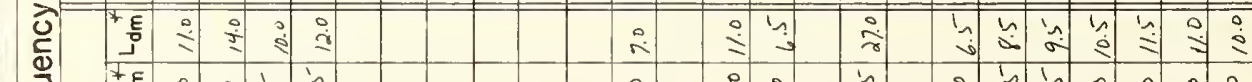

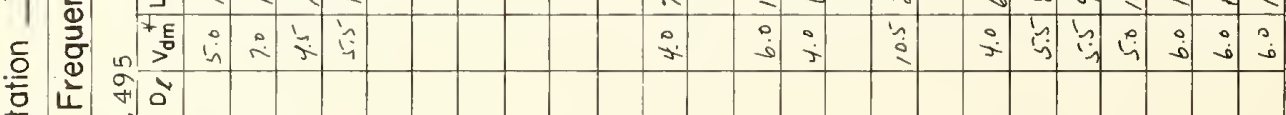

कह

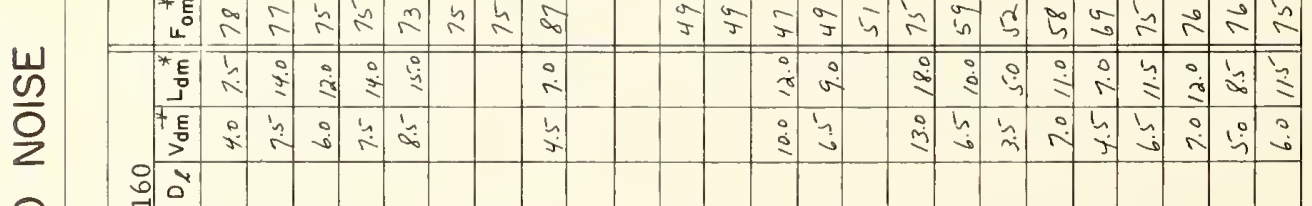

음

닝

$?$

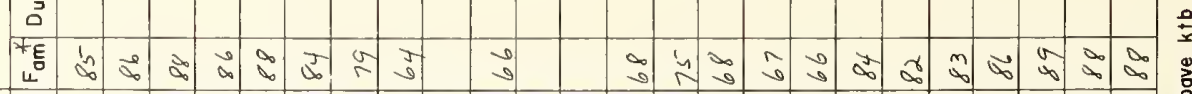

情

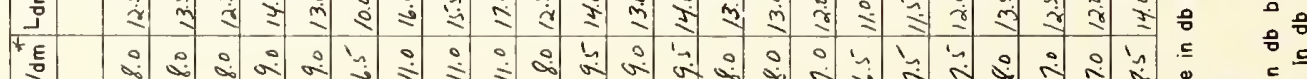

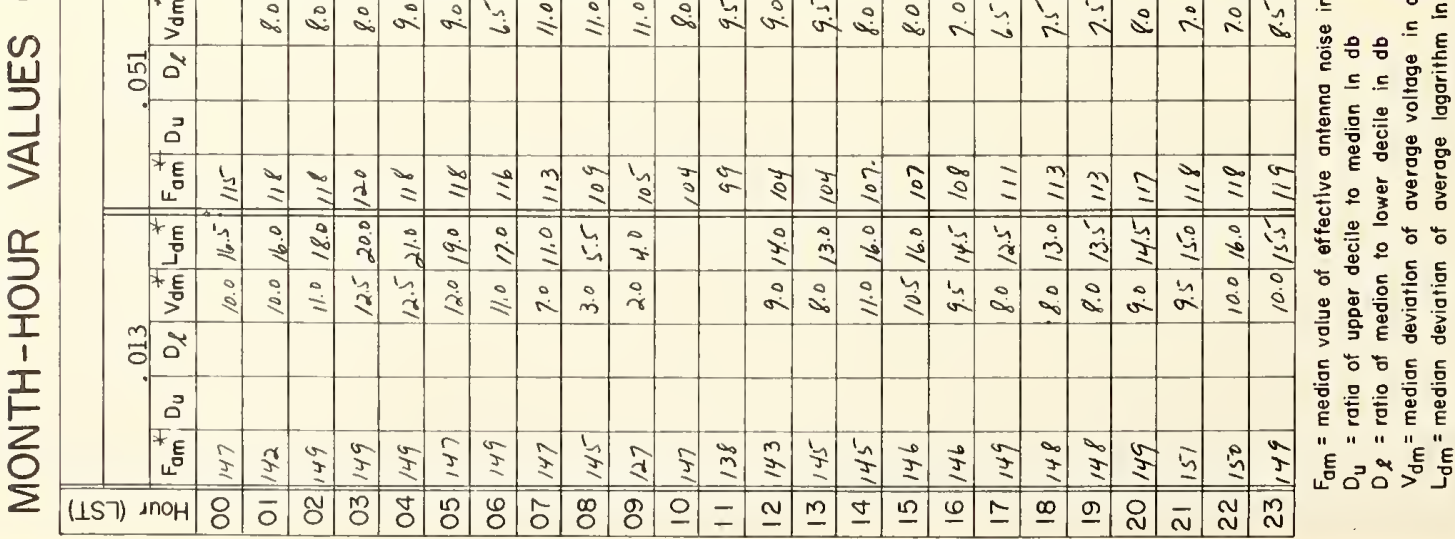




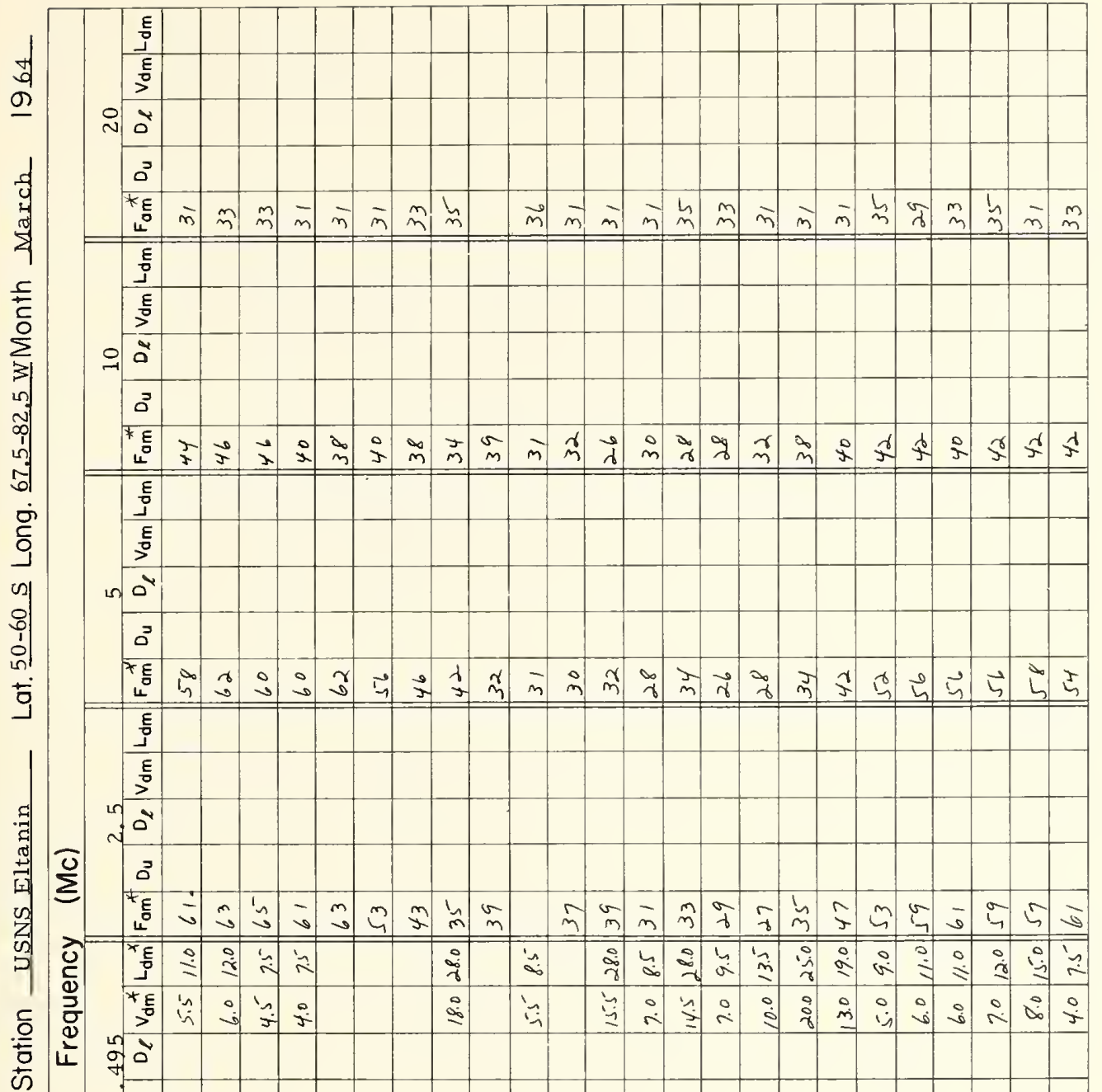

$0^{3}$

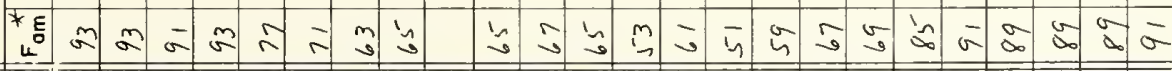

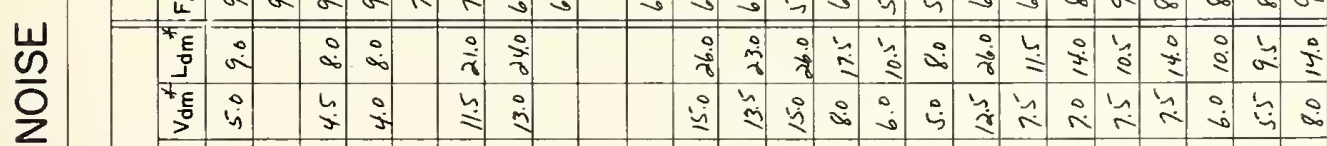

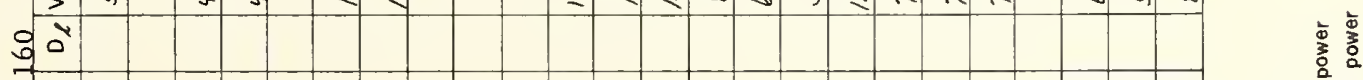

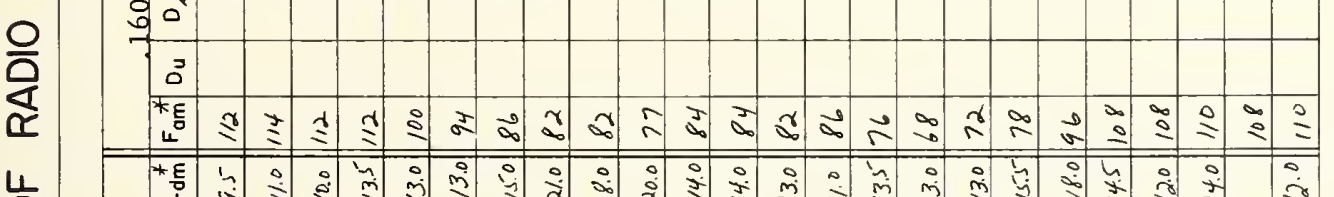

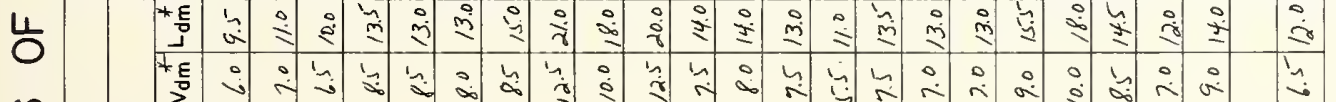

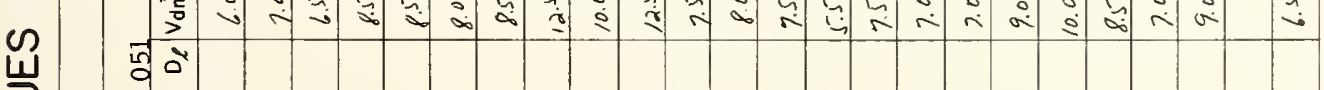

ב

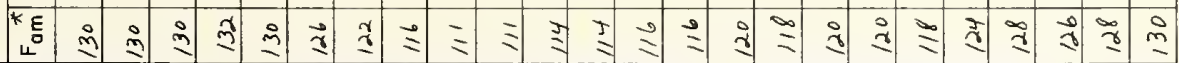

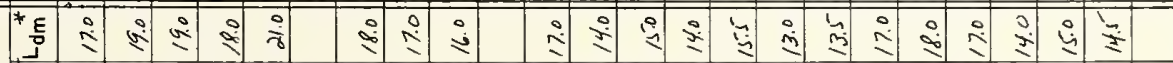

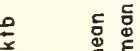

言势言

\&

든

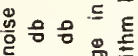

드드 总言

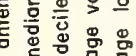

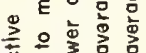

*E $\stackrel{m}{0}$

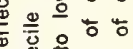

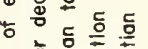

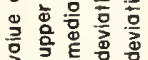

a

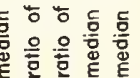

"

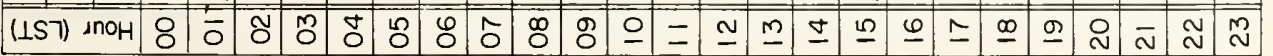




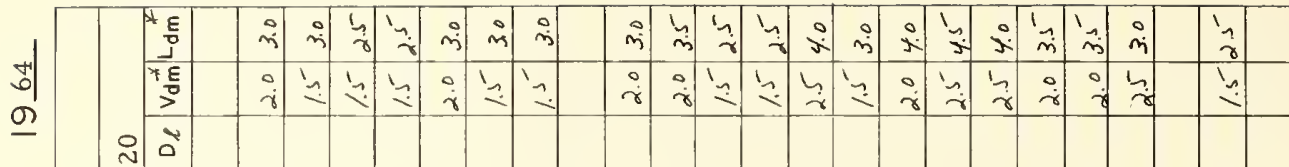

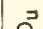

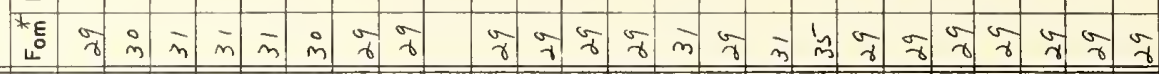

*

단

结

$\circ$ 음

$0^{3}$

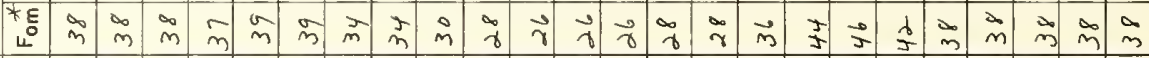

*

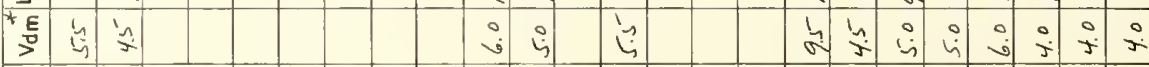

in $a^{y}$

영 in 0

疋

*E भी

+5

至

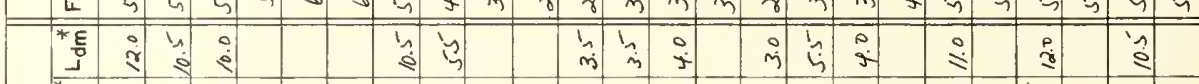

* E 0.0

约> $>0$.

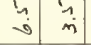

$\dot{r} \dot{\gamma} \dot{\gamma}$

$\hat{0}) \hat{s} \quad \hat{s}$

ง

U

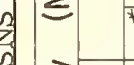

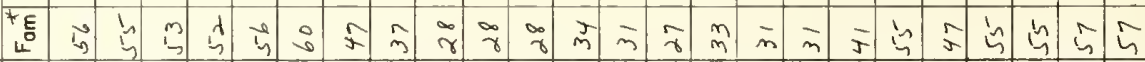

仓े

.들

늠

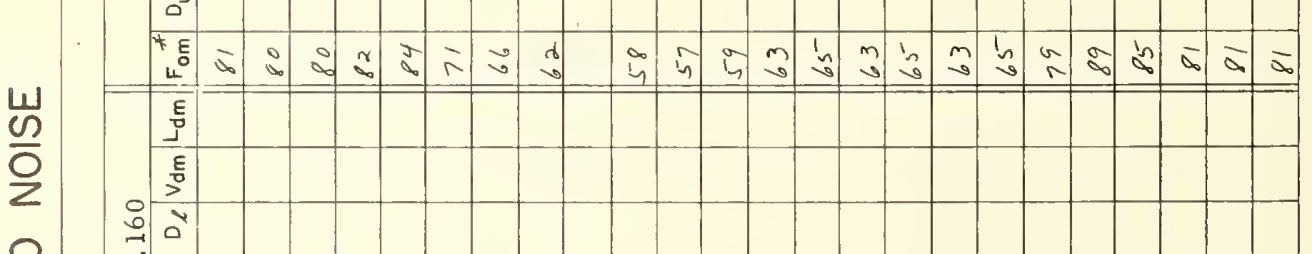

$\frac{0}{\square}$

ב

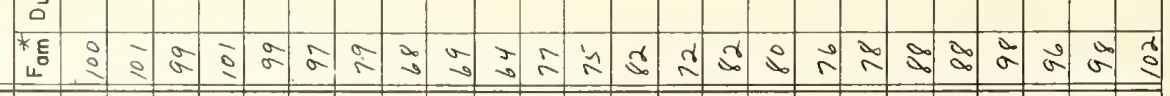

แั

E

$\stackrel{E}{>}$

岂

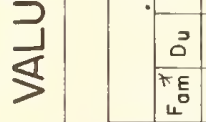

$\frac{0}{5}$
$\frac{1}{1}$
$\frac{1}{5}$
$\frac{1}{0}$

ह

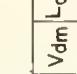

ฮั ชั

3

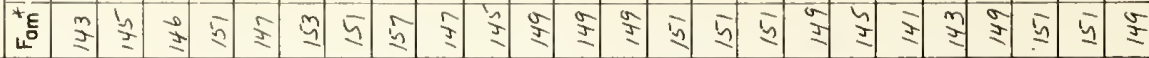
(IS7) यกон

言言

$\stackrel{5}{\frac{1}{x}} \stackrel{0}{\circ}$

(

要

의

을

훙 ㅇํㅇ

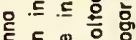

类 음

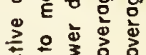

总 \%气

t。

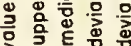

듷ㄷㄷㄷ

훙읗은

"En 


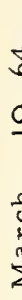

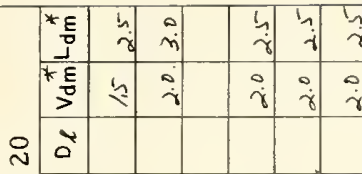

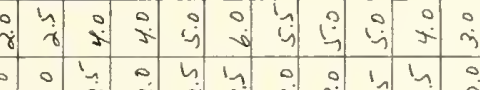

秃|

了

*E

†E

*E

$>$ ช

음ำ

$310-1$

(3)

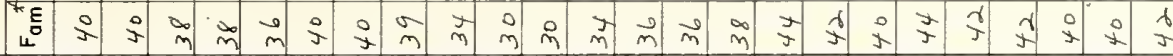

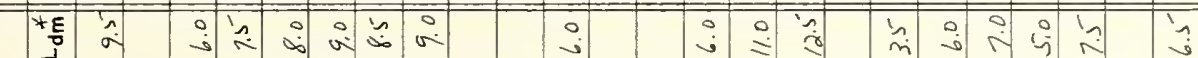

ॄ.

*E

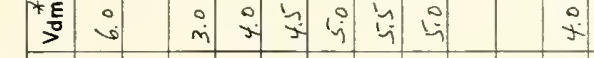

$\infty \frac{0^{2}}{3}$

*

*E जे चे

宁

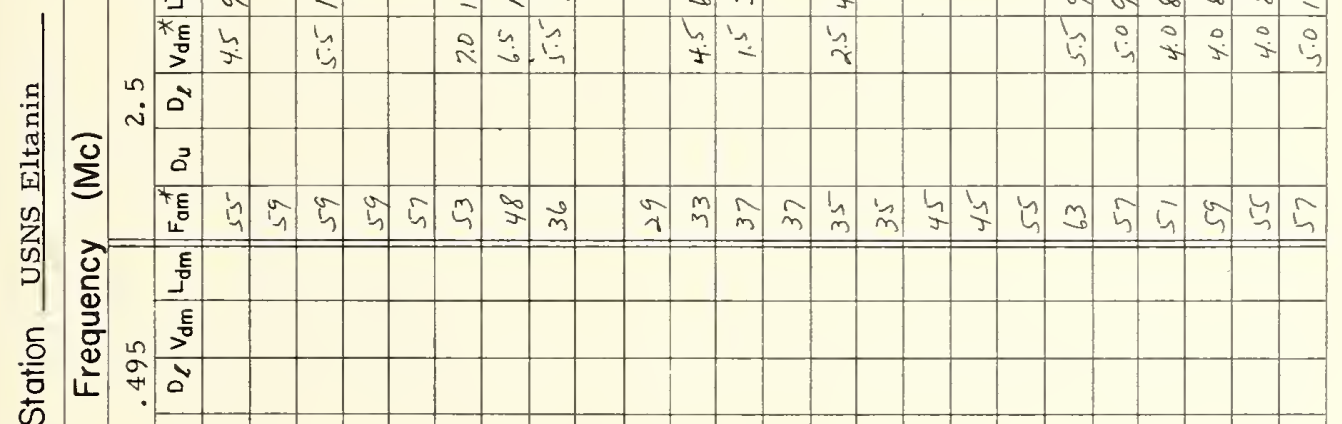

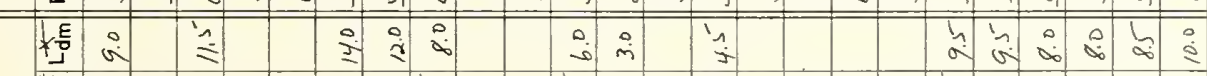

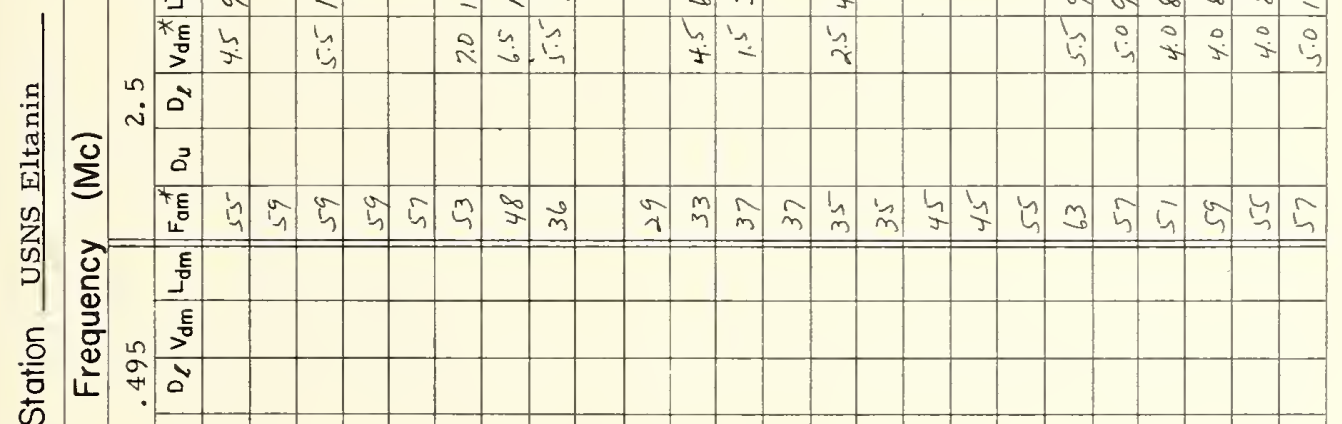

$a^{3}$

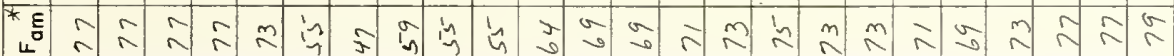

$\frac{\mathrm{w}}{\mathrm{O}}$

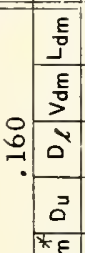

*

宩

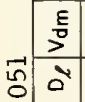

岂

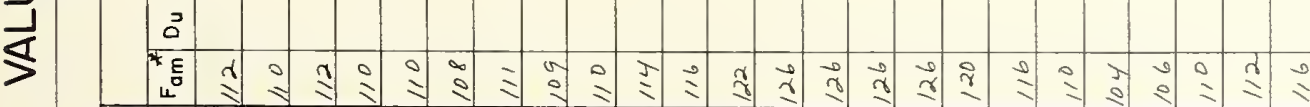

ह5

$\frac{x^{2}}{\frac{5}{5}}$

\begin{tabular}{l|l}
\hline \\
\hline
\end{tabular}

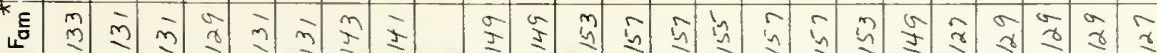

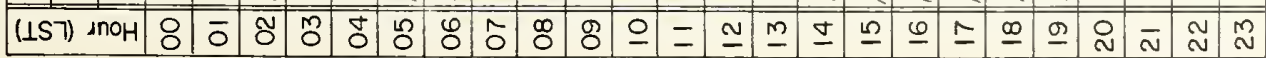




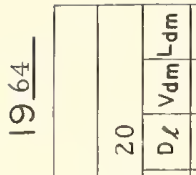

ना

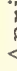

a

王

.

검

$\rightarrow$ 음

in

ปิำ

ธ్

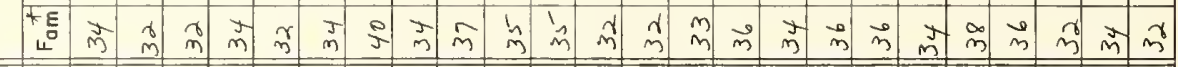
ह

פ্

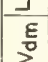

in $\ln 0^{x}$

잉

宁

3

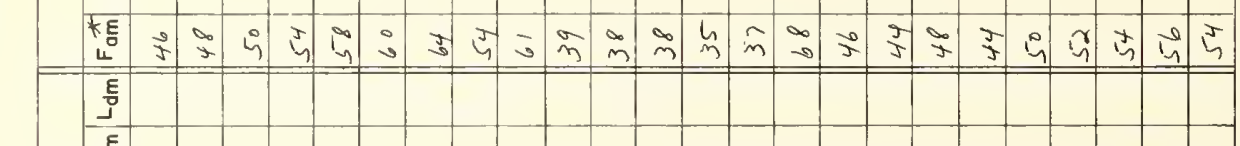

:

$\stackrel{n}{i} i^{2}$

D

设 $\times$

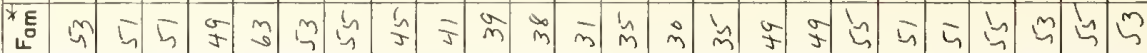

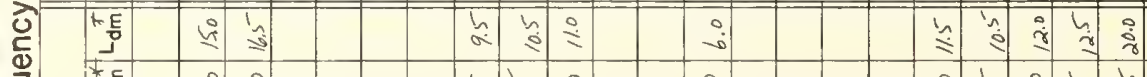

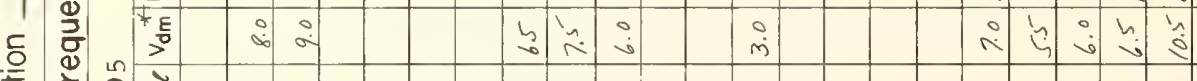

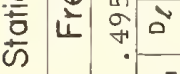

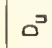

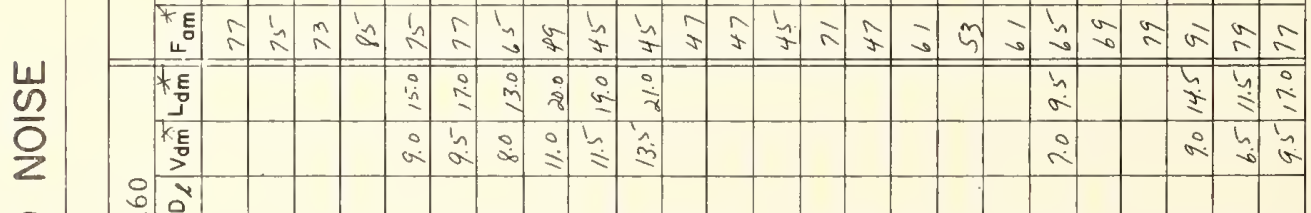

$\frac{0}{2}$

$\rightarrow 0$

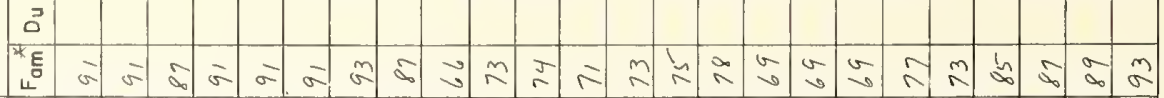
x

농

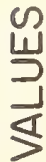

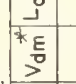

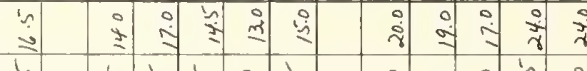

戈

in 0

उ

कह

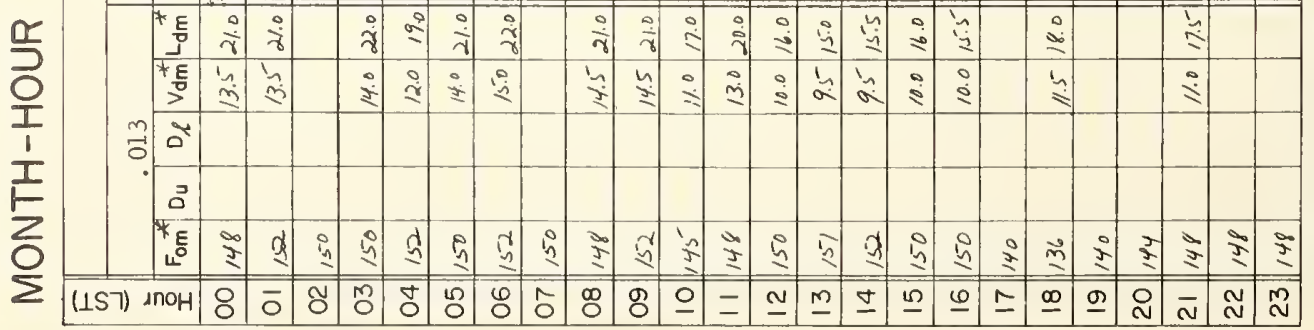




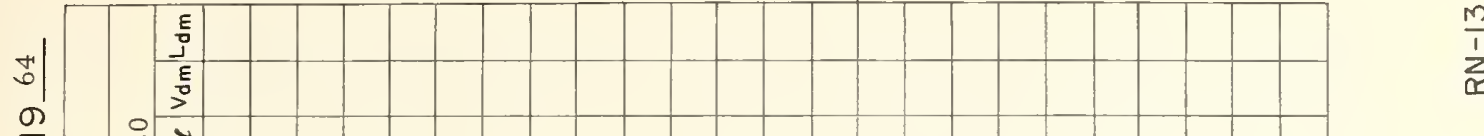

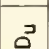

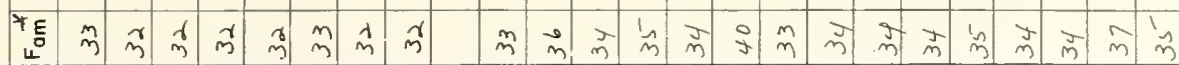

हE

$\stackrel{5}{ㄷ}$

责

$\circ \stackrel{\circ}{\circ}$

3

กิก

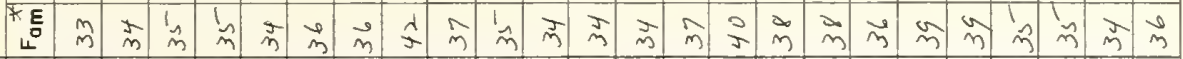

$\hat{m}$

ํํํ

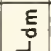

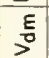

in $a^{4}$

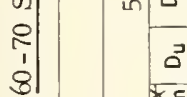

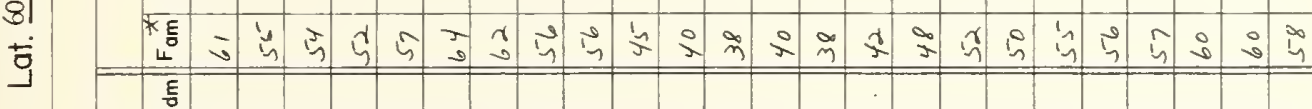

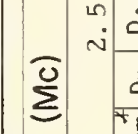

镸 m

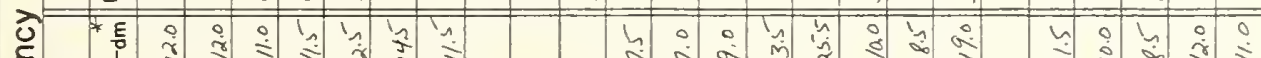

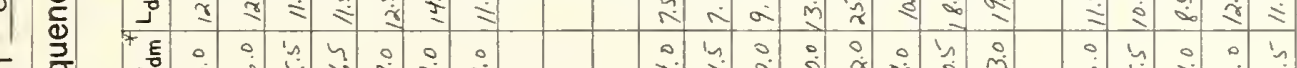

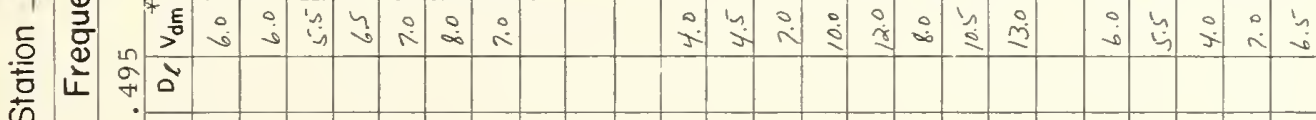

$0^{3}$

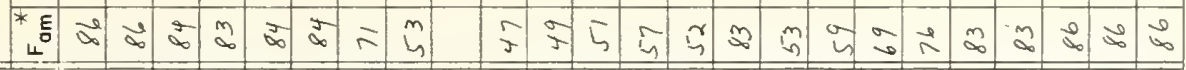

岂 ॠ

F

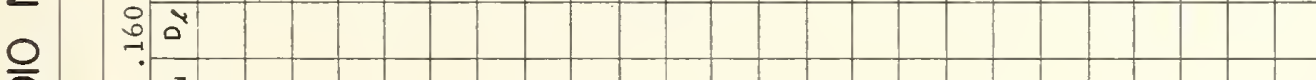

a

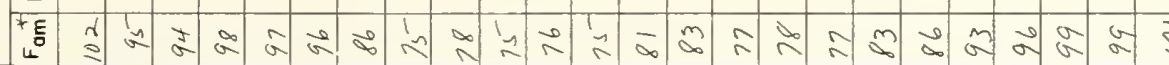

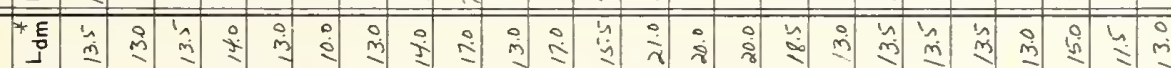

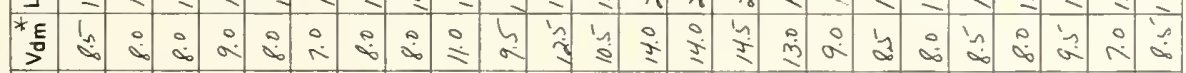

岂

*

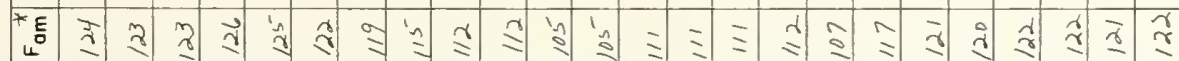

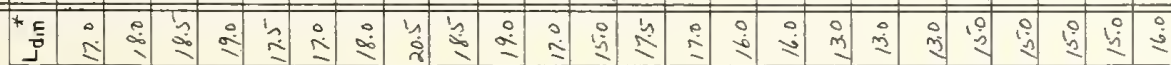

F, $\stackrel{m}{0}:$

声

* है 


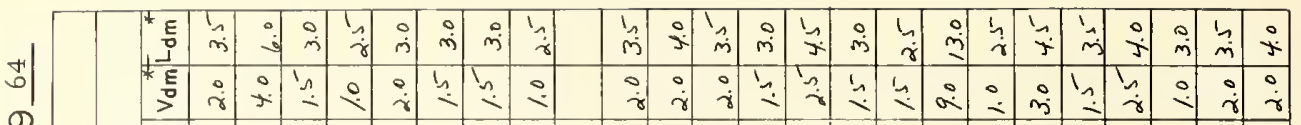
กิ้

a

苍

$\stackrel{\frac{\digamma}{c}}{\stackrel{c}{L}}$

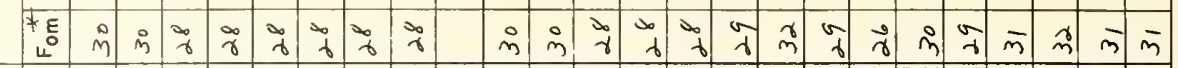

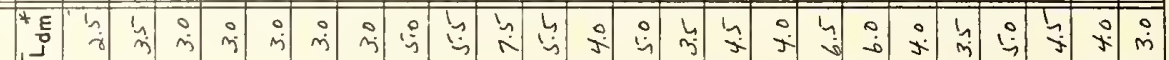

*

ำ

3

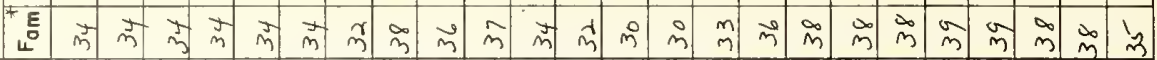

in:

ס्ञ

†E

*E

O'

궁

잉

宁

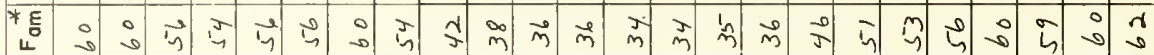

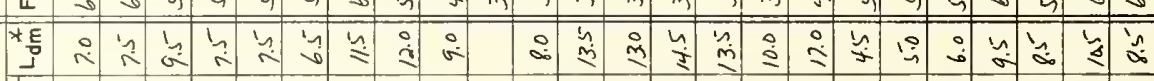

कE 0 F

:

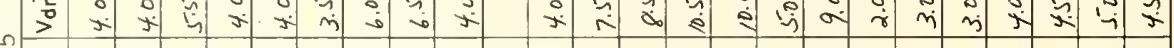

I

$>=$

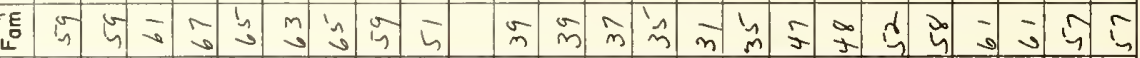

С.

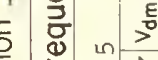

立 学䒕

$0^{3}$

ॠ

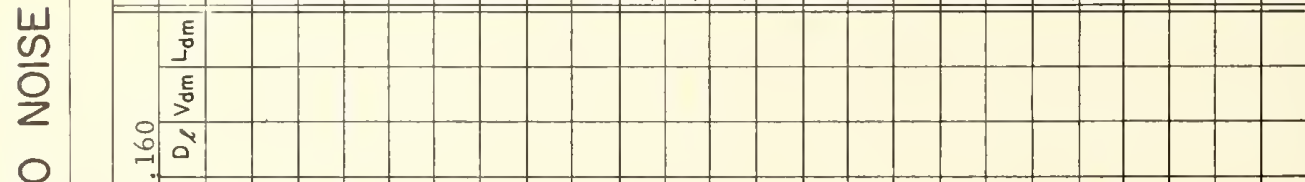

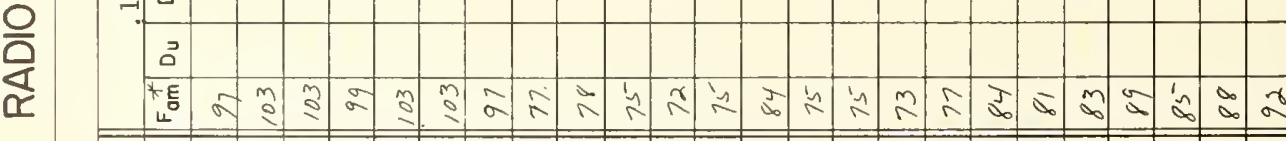

U

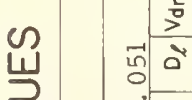

$\stackrel{1}{>}$

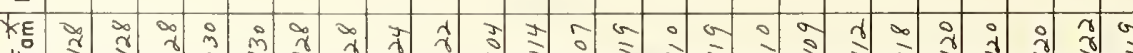

틈

$\simeq$
$\frac{1}{1}$
$\frac{1}{1}$
$\frac{1}{2}$
$\frac{0}{2}$

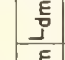

통

ढे ठே

ב

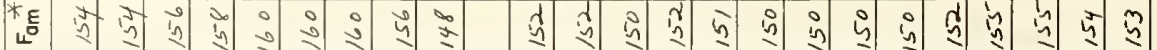
(157) มกํ 


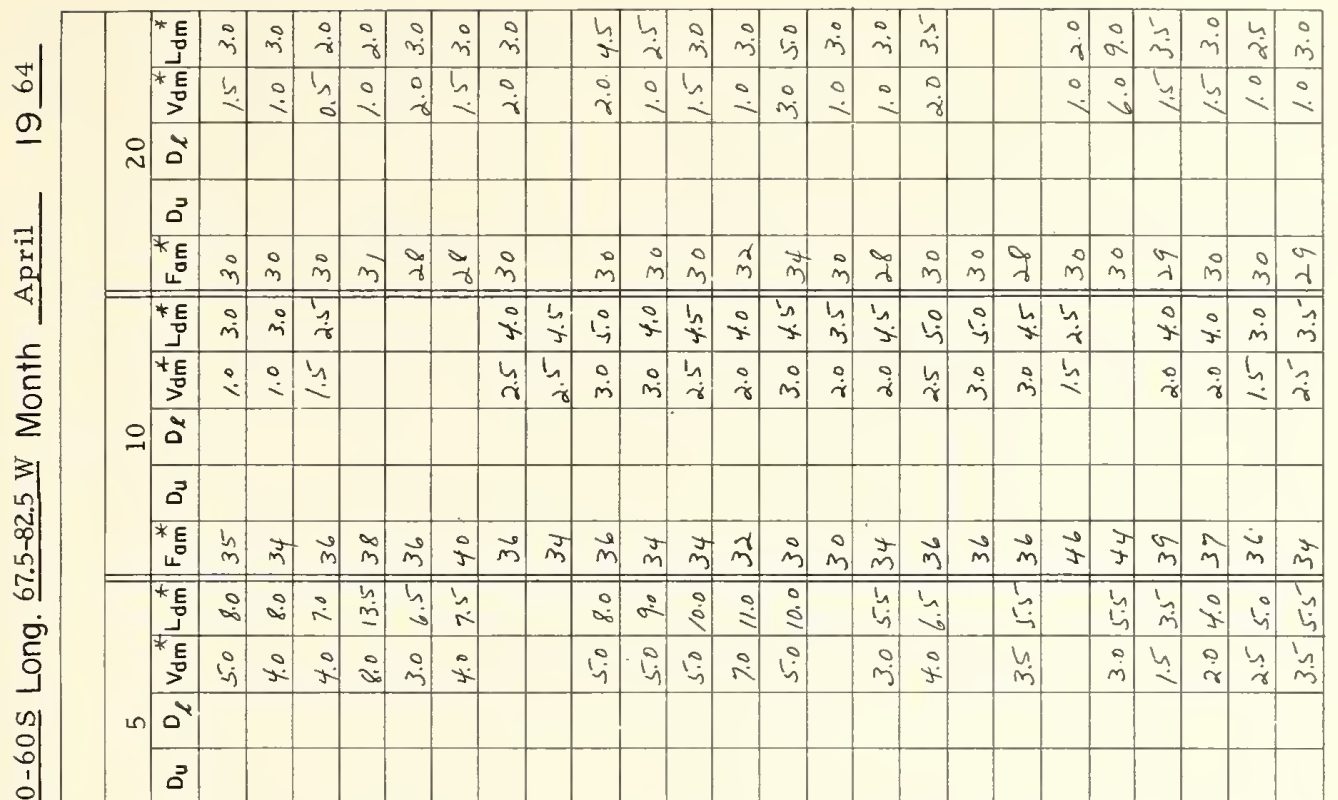

* ${ }^{*}$ i

$+\dot{0}$

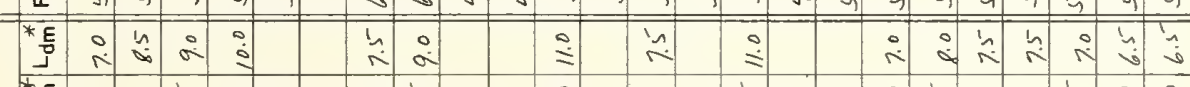

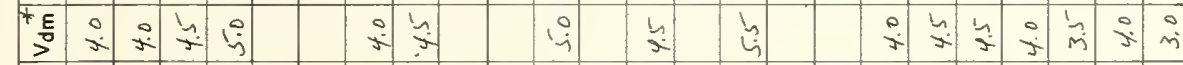
in

$\stackrel{0}{\stackrel{0}{\infty}}$

ב

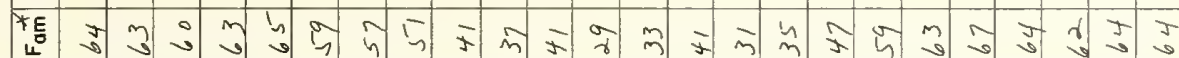
它 $\stackrel{\frac{1}{5}}{\frac{E}{0}}$

a

*E

$\frac{w}{2}$

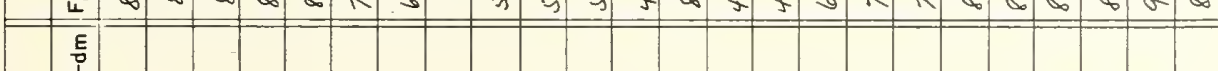

$\stackrel{5}{\frac{E}{5}}$

일

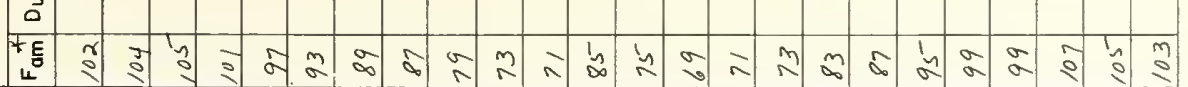

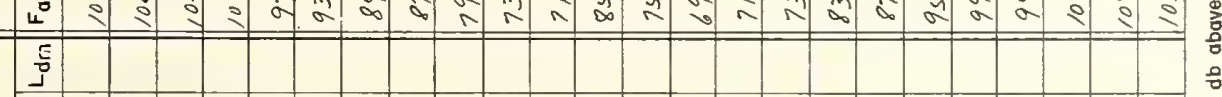

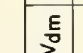

$\overrightarrow{0}$

בे

*

톰

चै

ב.

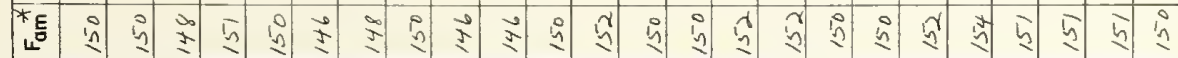

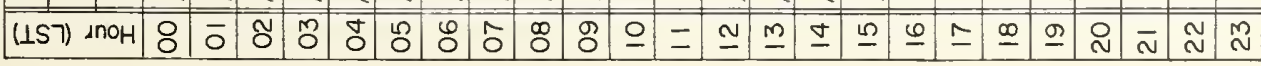

害言

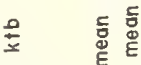




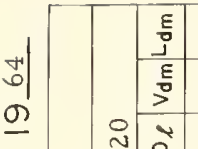

$-1 \mid$

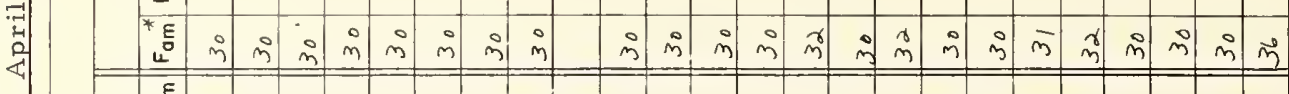

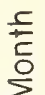

皇

ह

음

3

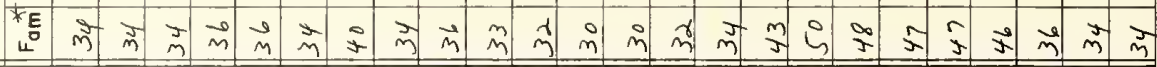

है

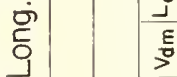

in

3

에

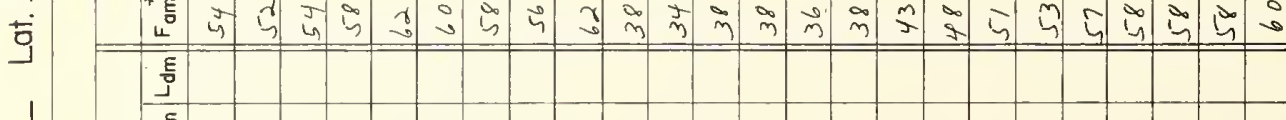

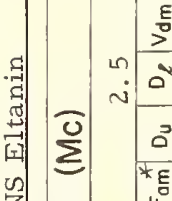

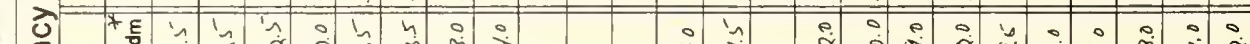

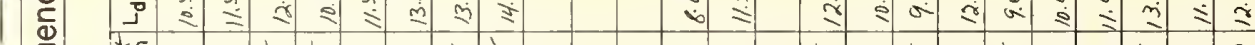

ᄃ

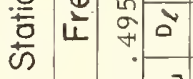

0

ॠE

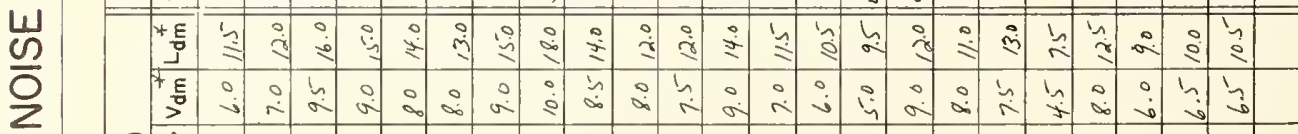

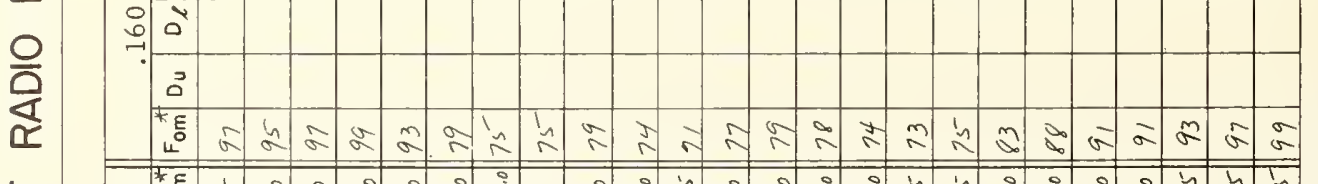

L

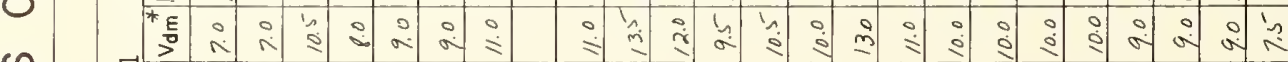

$\underset{3}{3}$

in $>$

3 .

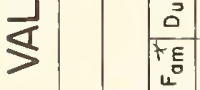

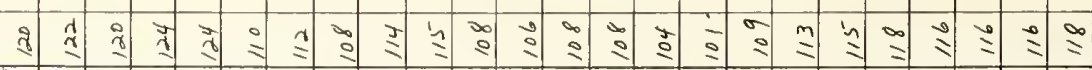

*E

$\frac{0}{5}$

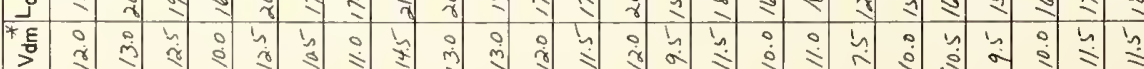

$\frac{1}{1} \quad \stackrel{m}{0} 0^{r}$

I

(LSา) มก 


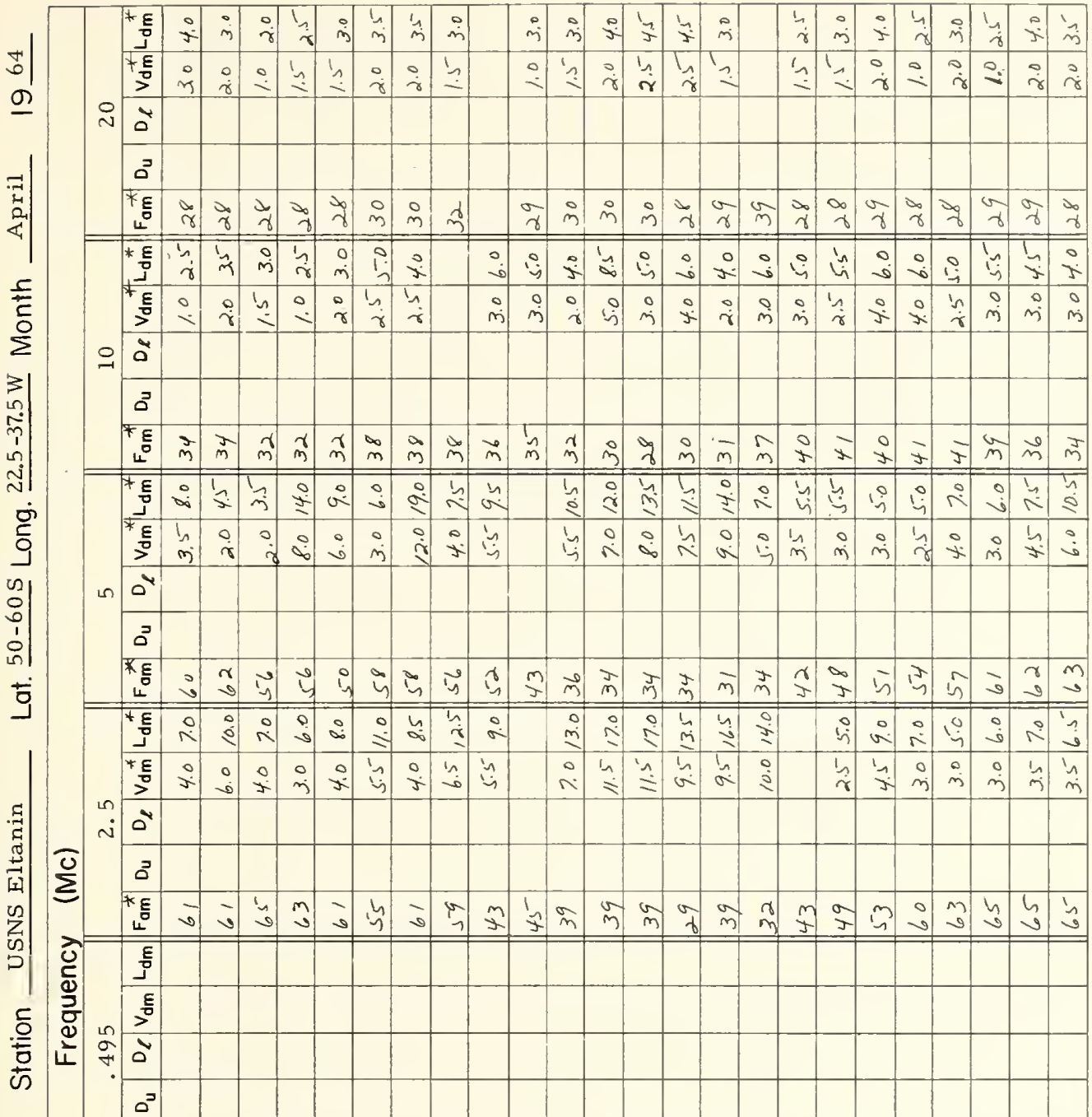

*

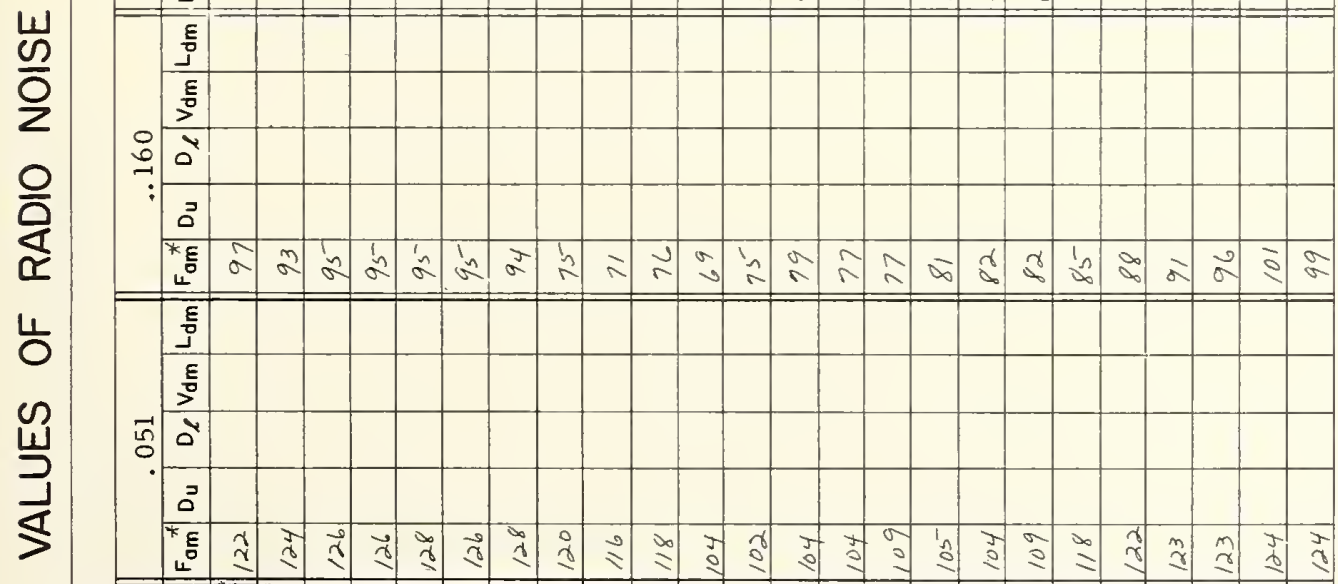




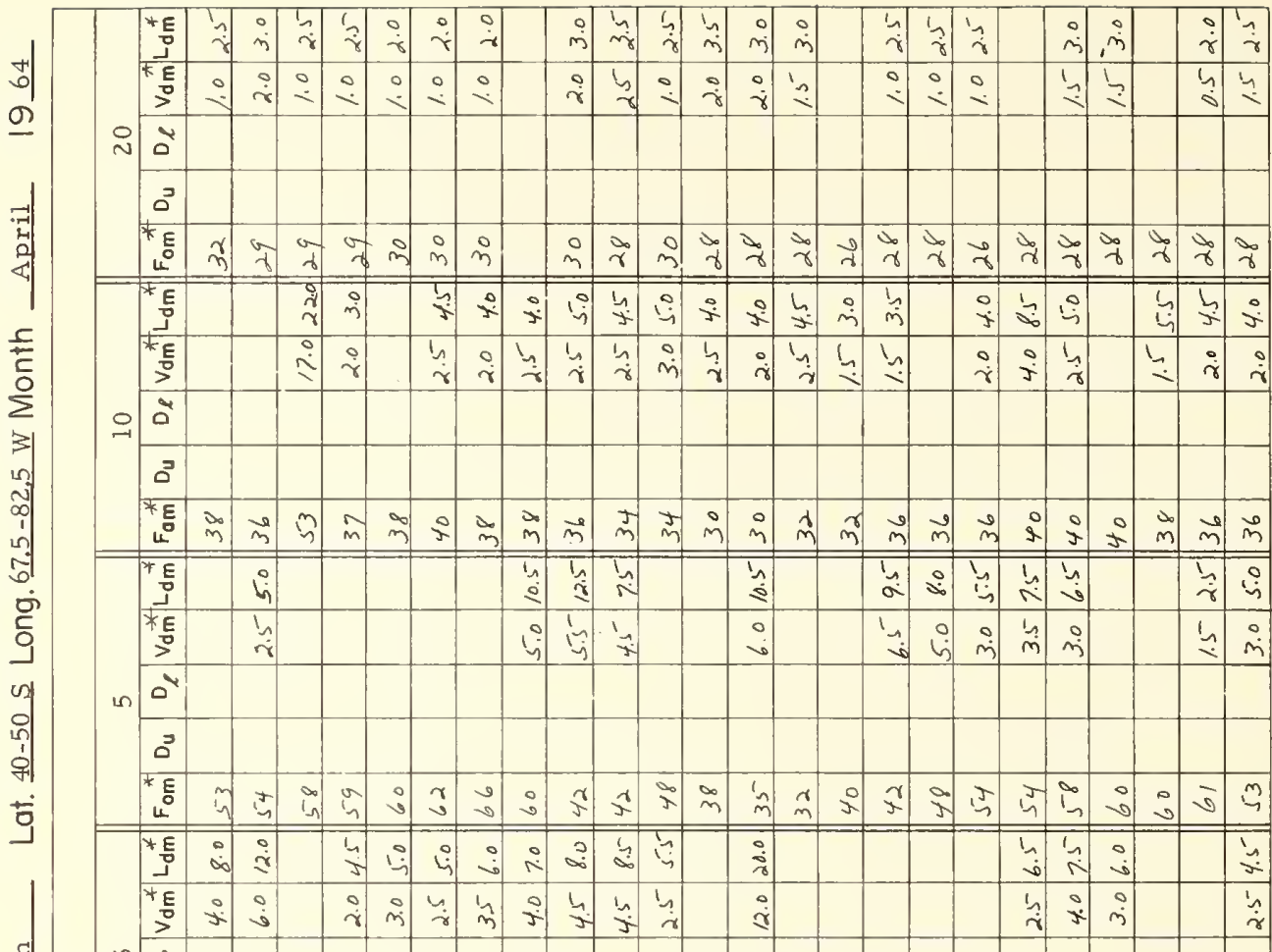

:

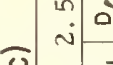

$\sum$

*E

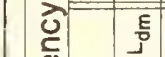

ᄃ

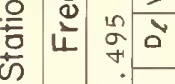

3

*E

峞

$$
\text { 늘 }
$$

들

임

응

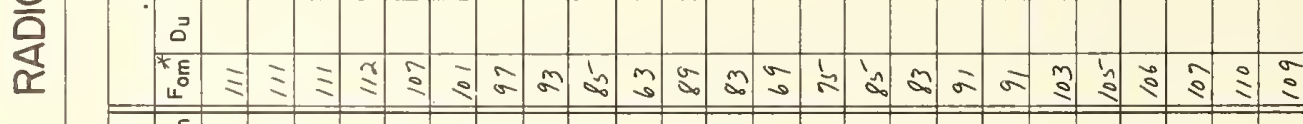

논

E

in

5 出

$\frac{1}{5}$ a

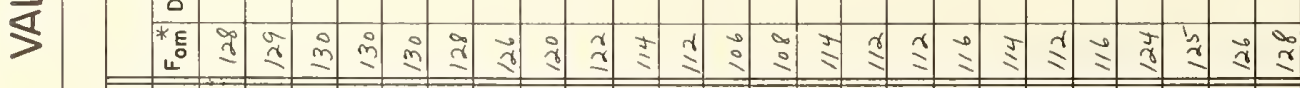

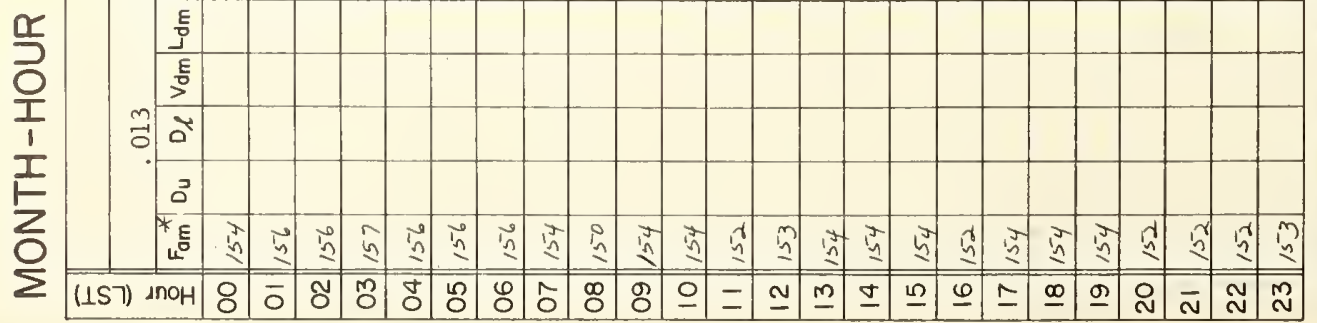

高高 
ㅇํㅇ

뵨

3

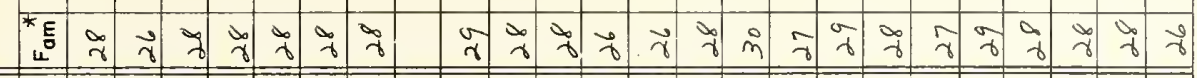

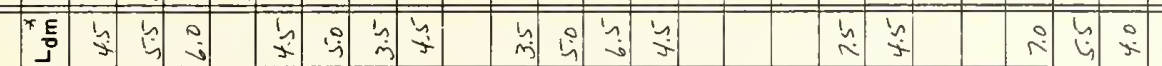

営

F

웅

a

Tह

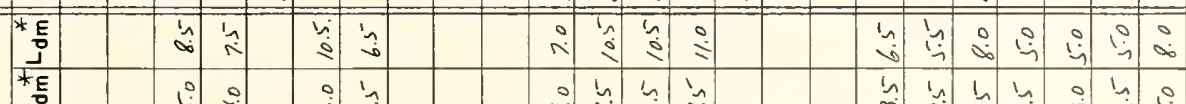

$>$

i. :

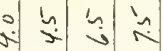

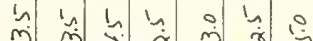

in.

$a^{3}$

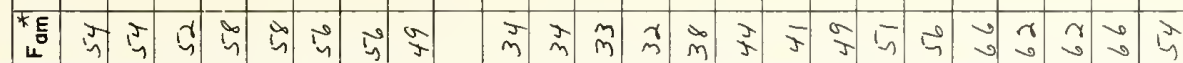

+ह

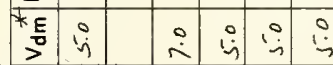

离

is

3 in

$\sum^{0}$

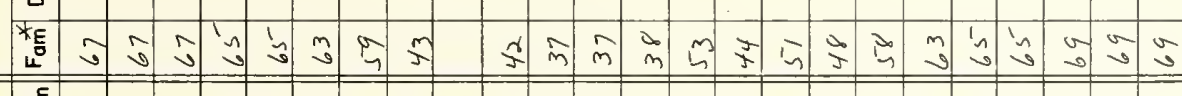

亭

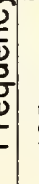

E

岁

$a^{3}$

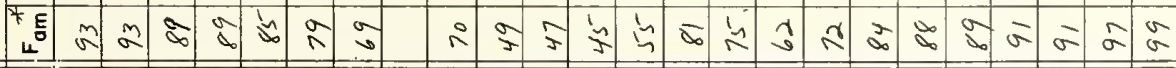

荝

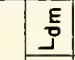

\begin{tabular}{ll|l}
\hline \\
\hline
\end{tabular}

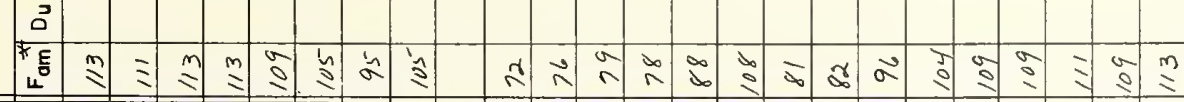

통

竞

तू

כ

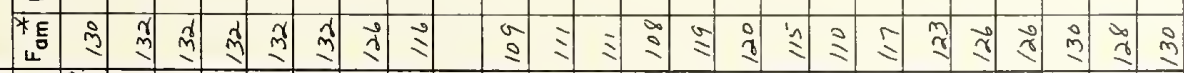

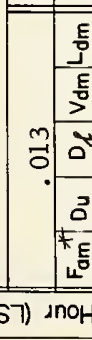

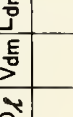

ב.

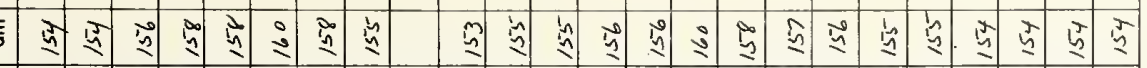
(LS7) 2noH 8 б 


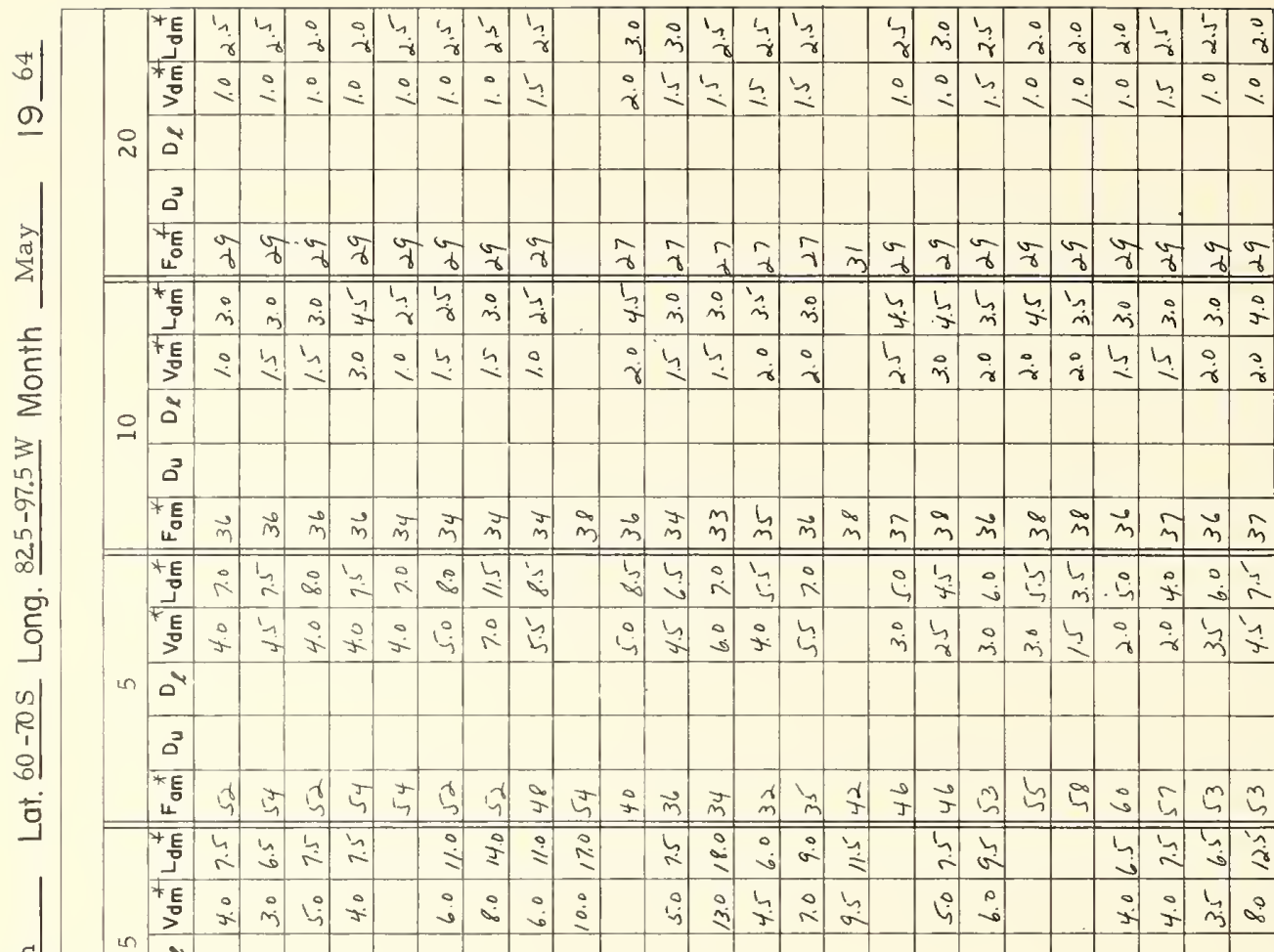

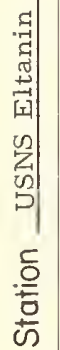

$\sum^{0} \quad 0^{3}$

i व

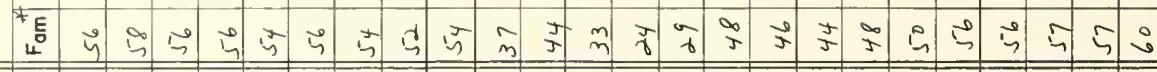

ठे

要 $\ln \frac{2}{2}$

14 市

$0^{3}$

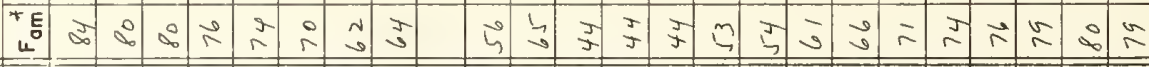

$\frac{41}{2}$

E

E

$\stackrel{0}{0} 0^{2}$

은

๘

व

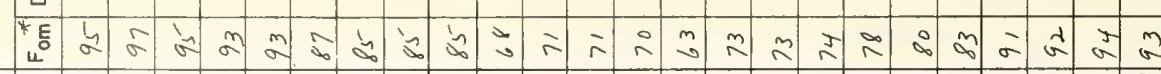

แ山

E

E

$\stackrel{\omega}{5} \quad$ :

a

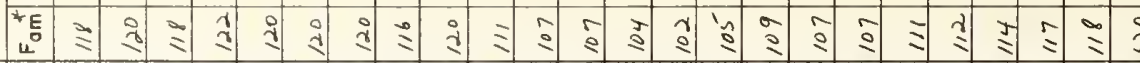

0
$\frac{1}{0}$
$\frac{1}{1}$
$\frac{1}{2}$
$\frac{1}{2}$

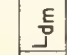

틍

कै ธ

a

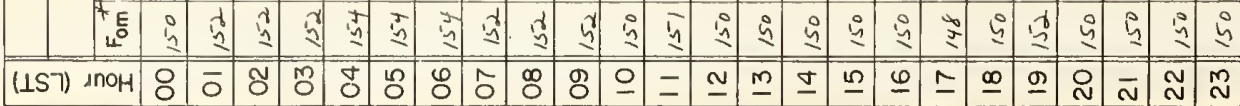

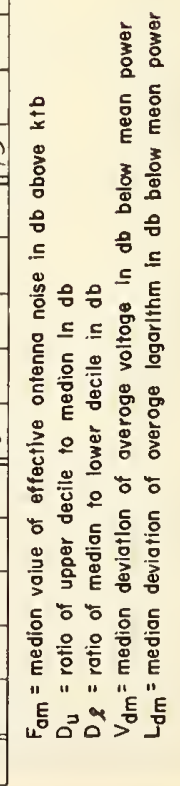


ำ

离

달

3

ॠह ज्ञ

독

资

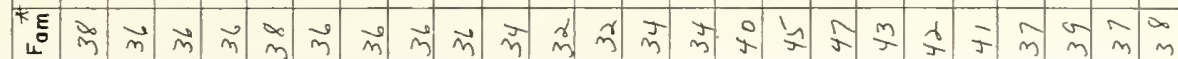
i.

官

\section{E}

$\frac{E}{>}$

n) 20

官

ᄒே

$0^{3}$

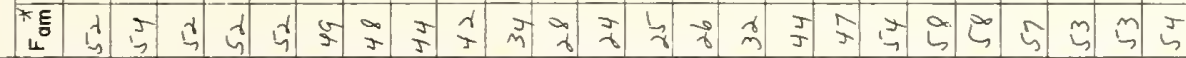

$\frac{E}{\frac{E}{J}}$

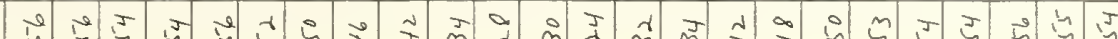

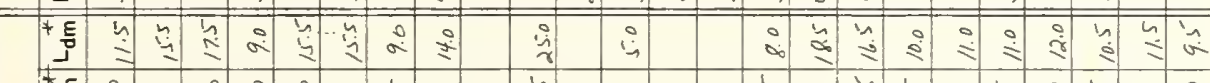

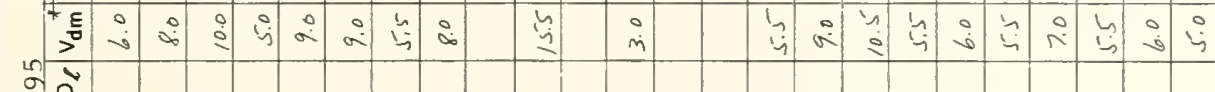

\%

$0^{3}$

ॠ

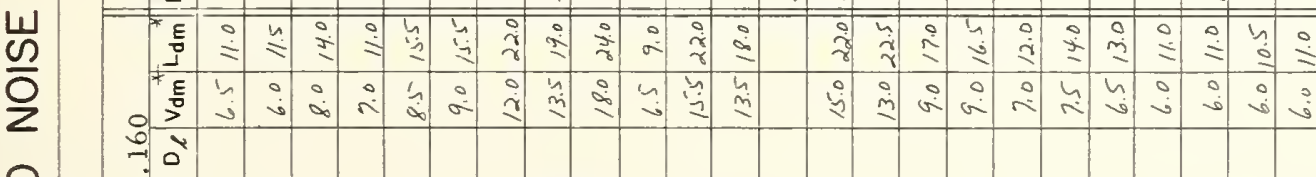

3

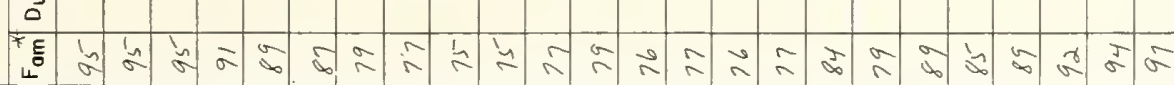

ॠ

*E

岁

כ

* ${ }^{*}$ )

*

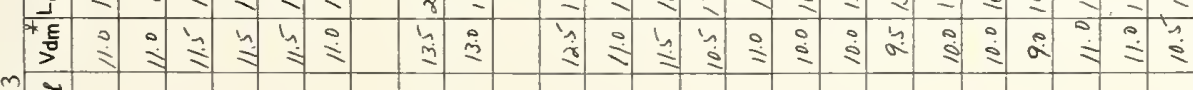

$\stackrel{m}{0}$ ठั

3

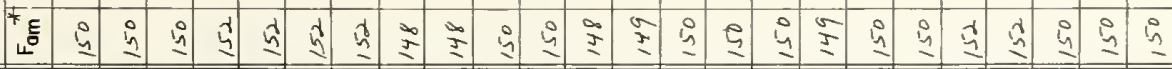

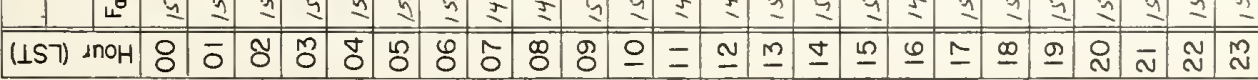




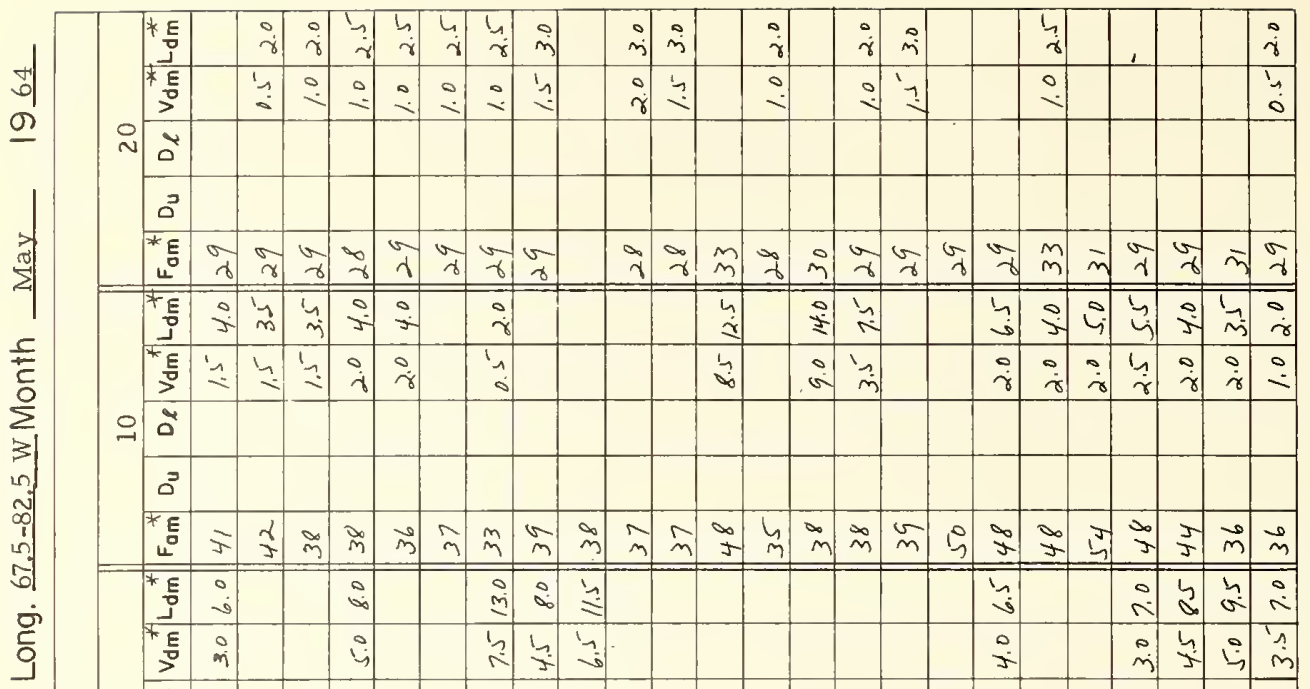

in $0^{x}$

ț

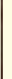

ن

o 0

3

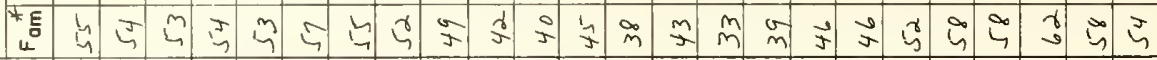

苛

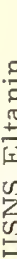

.

负 $=L^{\circ}$

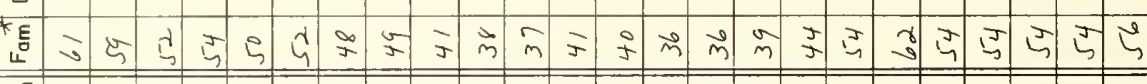

등

등

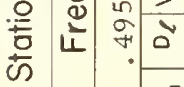

0

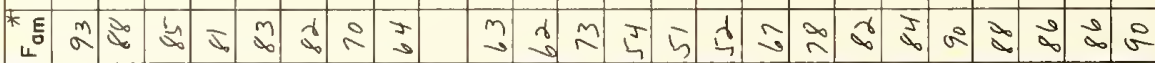

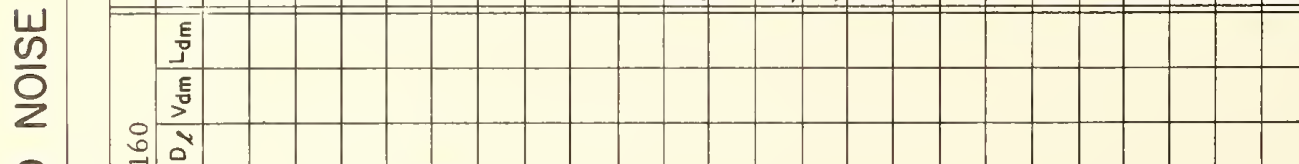

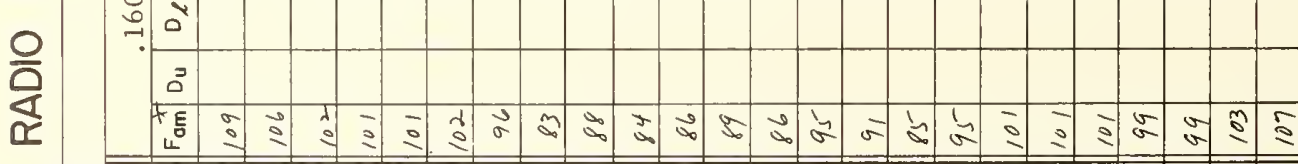

낭

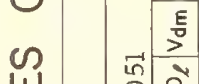

แ

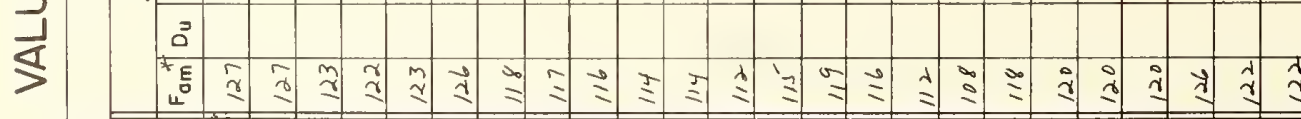

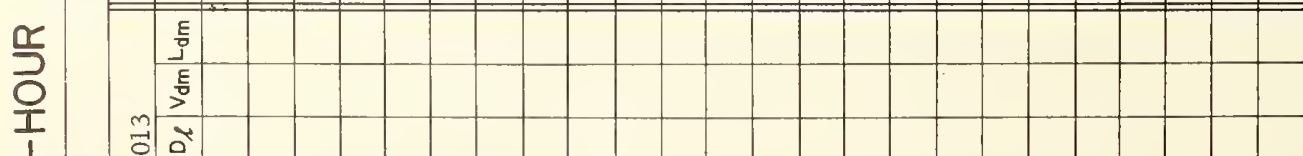

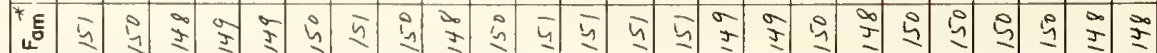
(1S7) มก, 


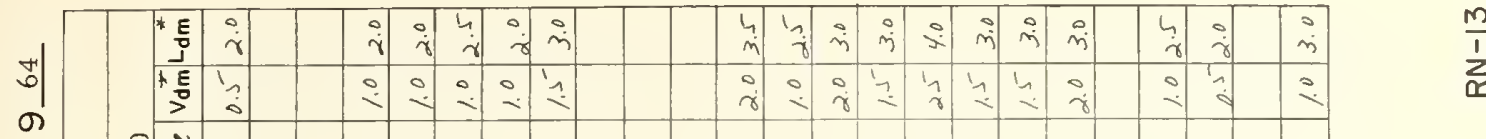

ㅇํำ

○

곡

*E ज્ર

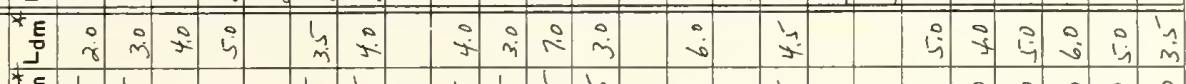

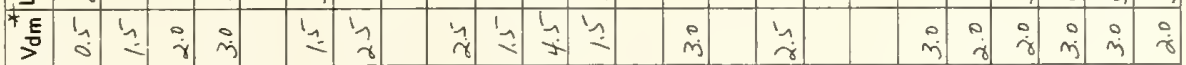

$\stackrel{\circ}{\circ}$

3

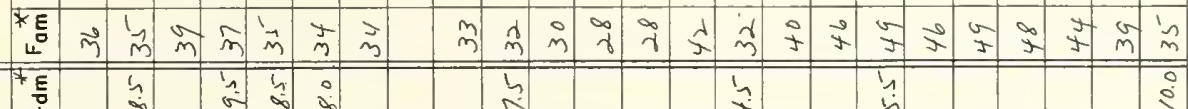

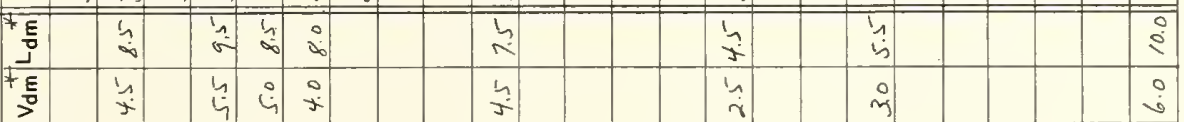

宮

$0^{4}$

in

0

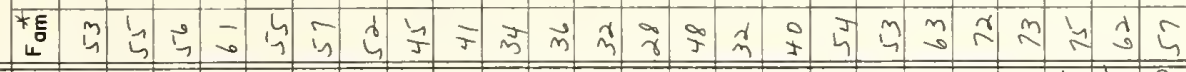

*

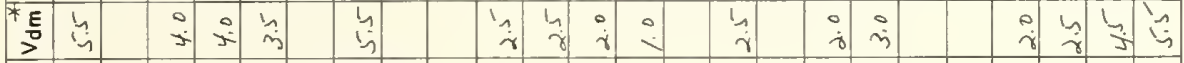

苞|

०

ב

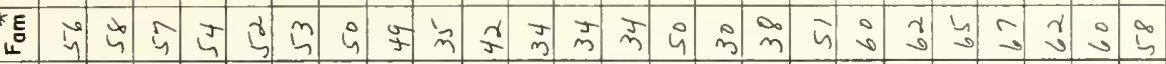

瓷

(

층

E

등 응

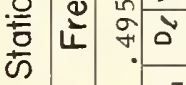

0

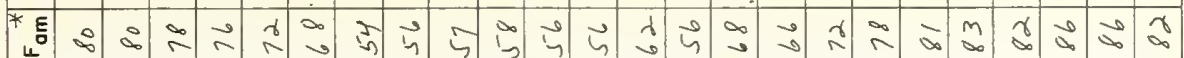

$\frac{1}{5}$

응

$+$

E

焉

:

कह

E⿱

秥

$\frac{E}{>}$

․

3

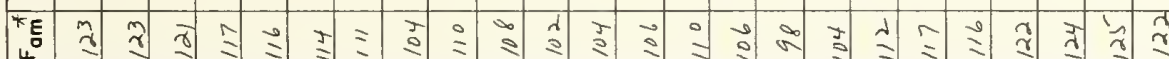

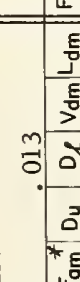

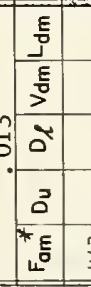

(เง) $1 \mathrm{AnOH}$ : 
3

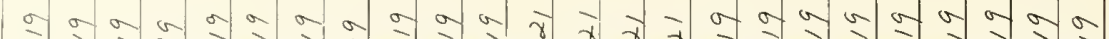




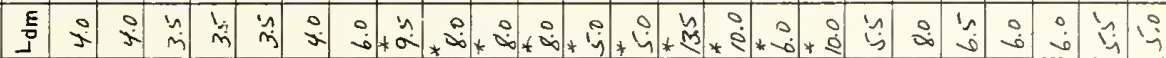

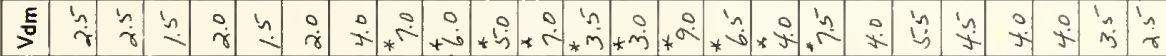

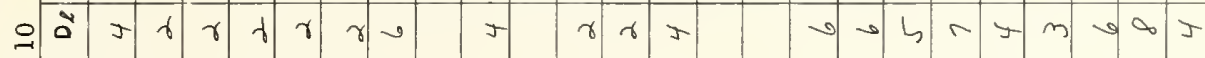

a $3 x y+y \rightarrow \rightarrow$ t

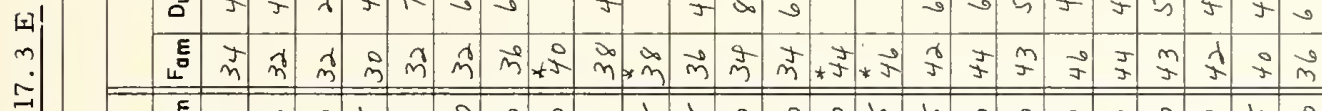

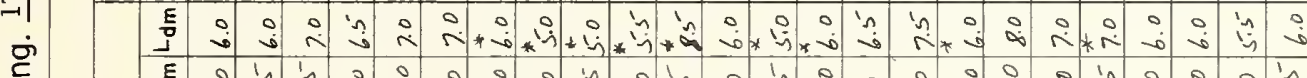

ㄷํㅇ

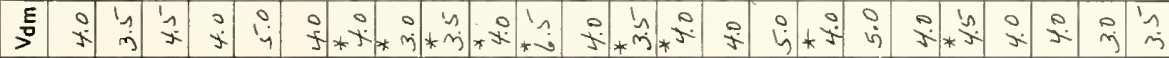

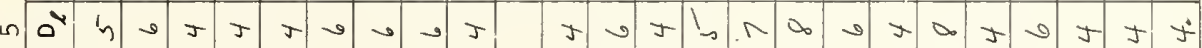

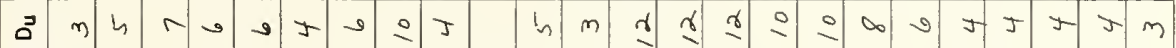
ह

*E * E a $\infty$ o

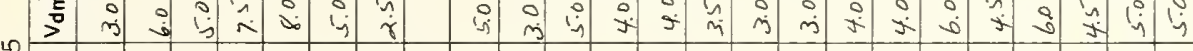
i d

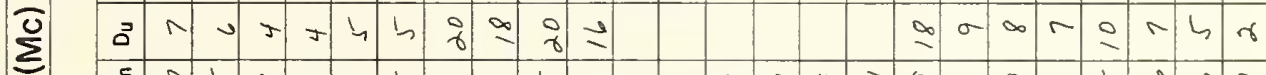

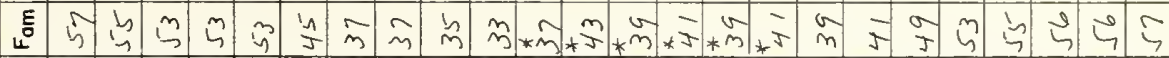

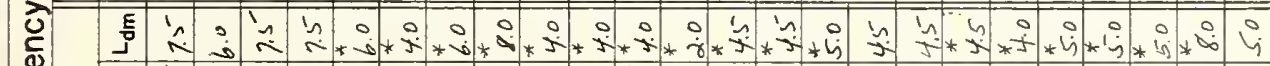

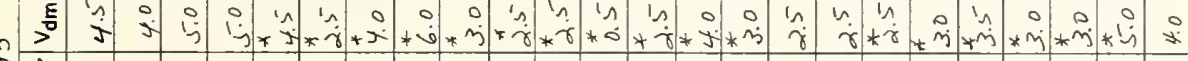
क्षे

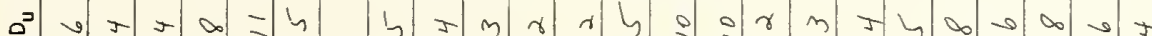

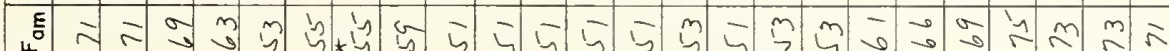

*E *E *E 8 वर

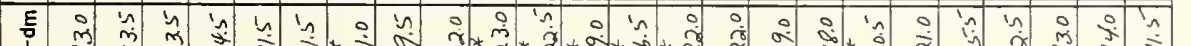




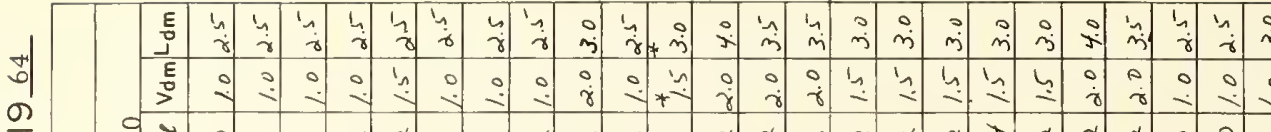

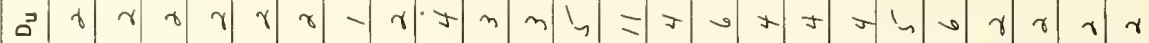

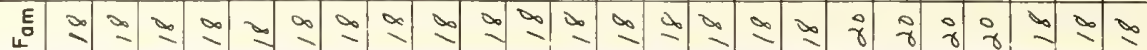

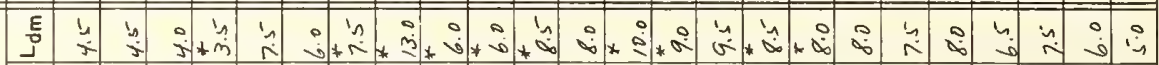

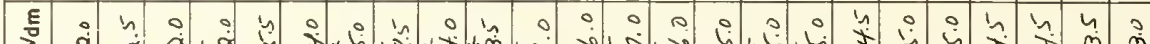

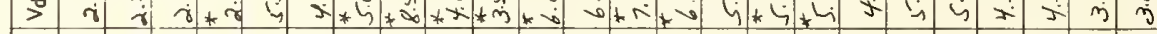

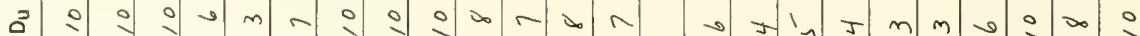

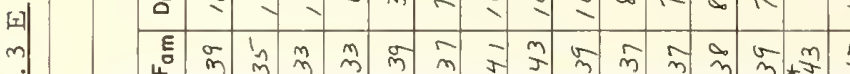

E

E

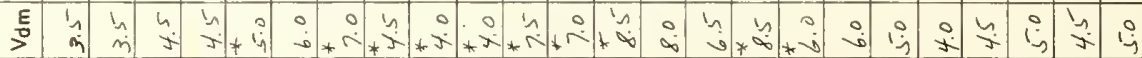

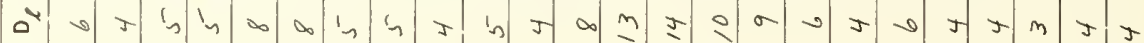

娄

हू

+

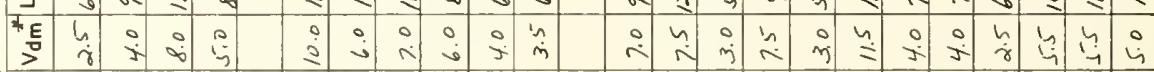

昰

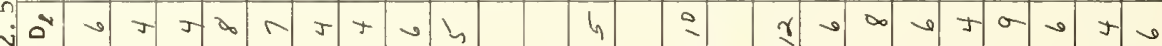

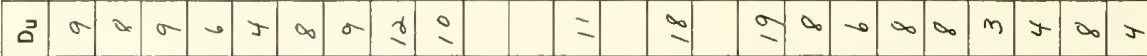

E

ठे E

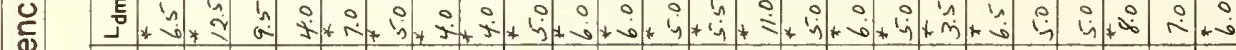

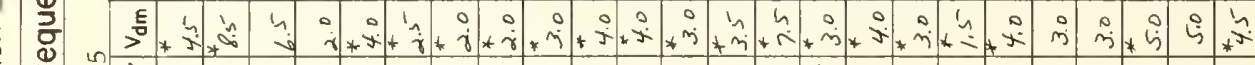

羊

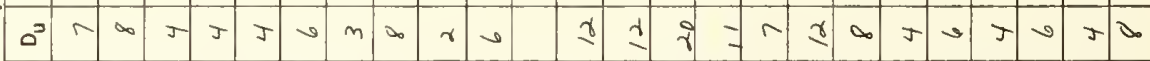

है

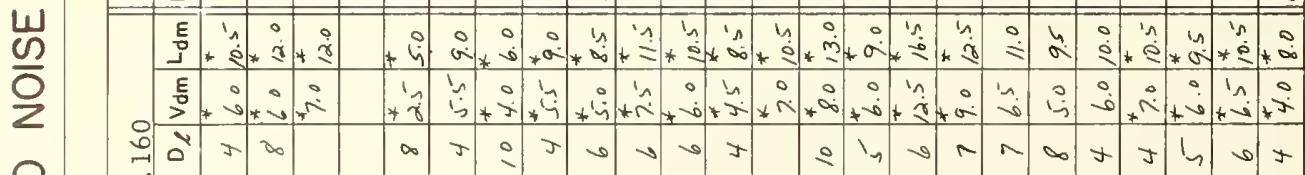

$\frac{0}{8}$

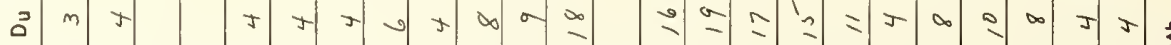

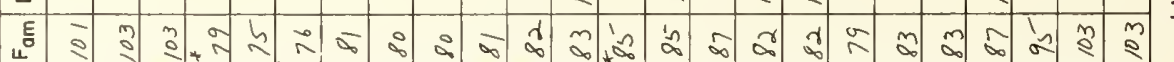

E

4

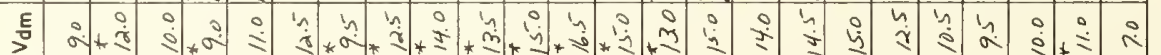

岃

फ जी

ב

E

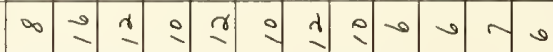

E हE

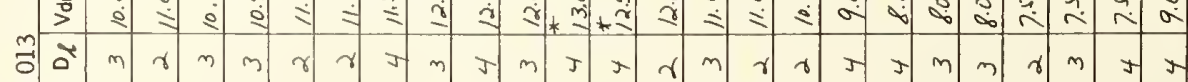

$\frac{1}{5}$
$\frac{1}{1}$
$\frac{1}{1}$
$\frac{1}{0}$ 


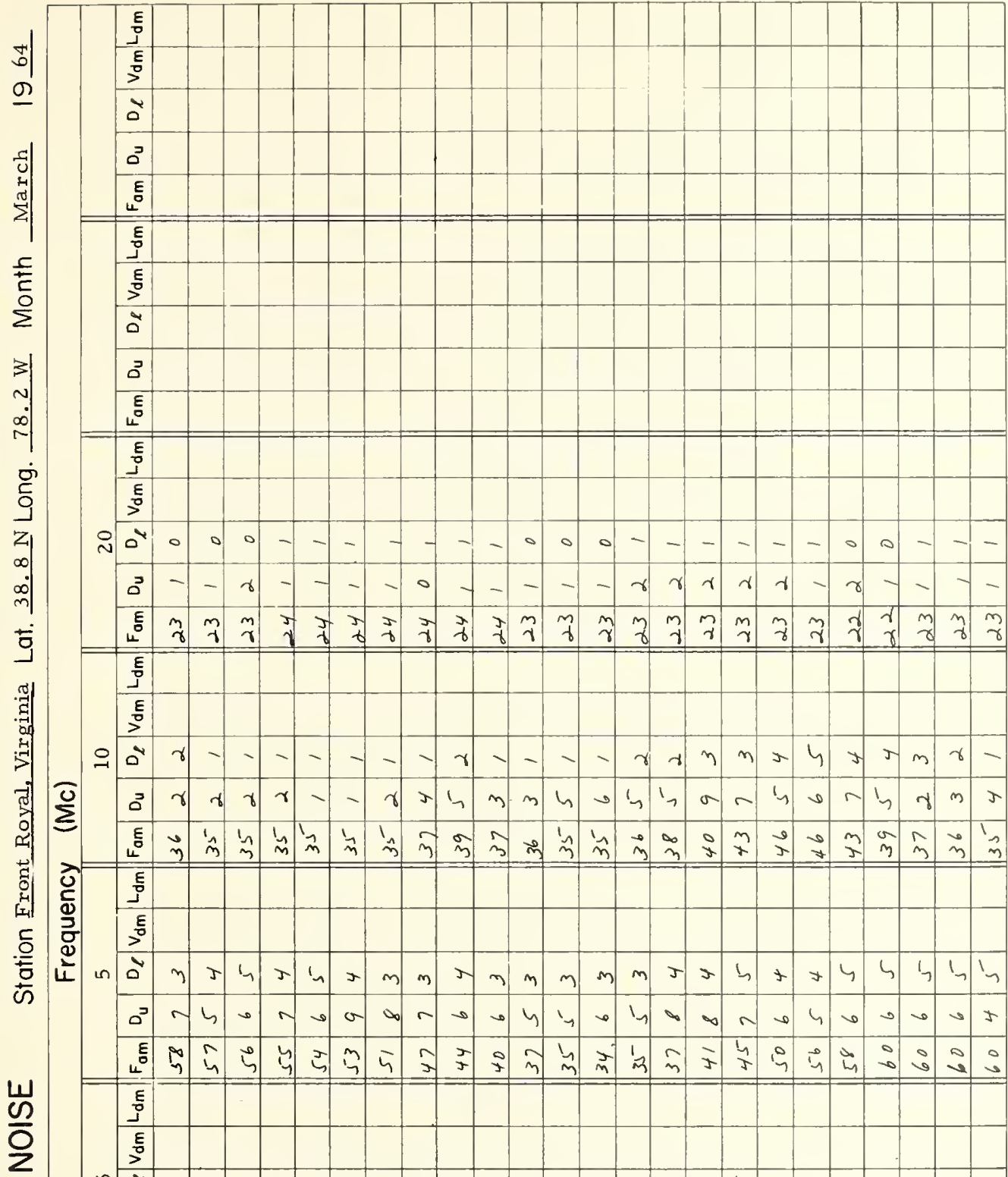

iñ

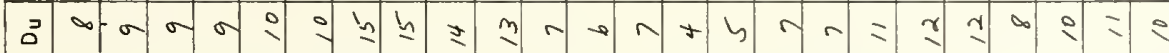

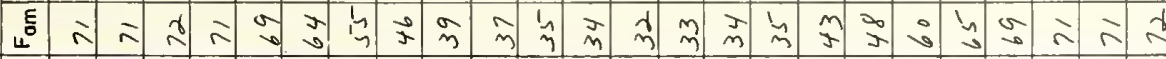

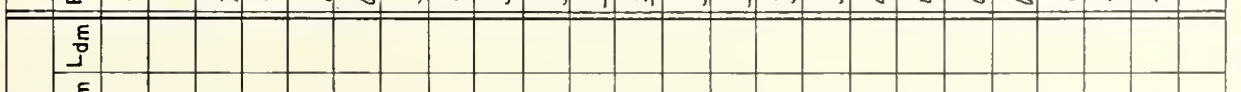

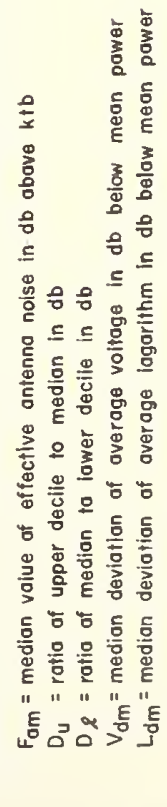




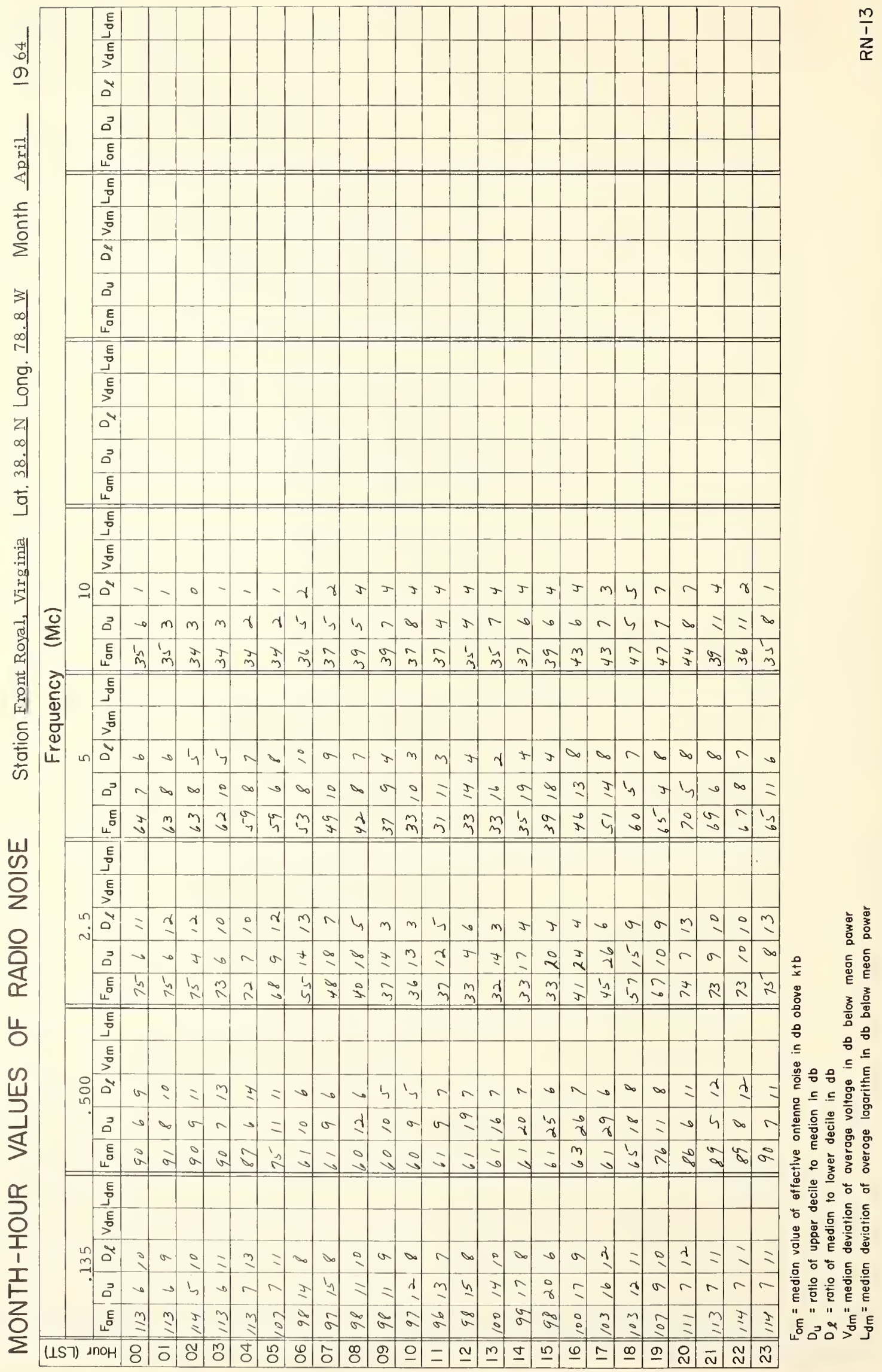




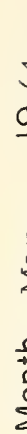

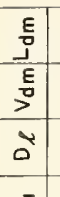

离

$a^{3}$

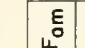

E

\begin{tabular}{|l|}
\hline 5 \\
\hline 5 \\
\hline 5
\end{tabular}

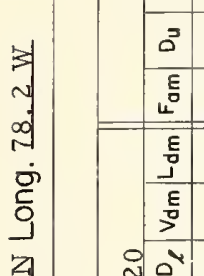

年

$m$

官

|a:

D

E 1 Е 皇

竞

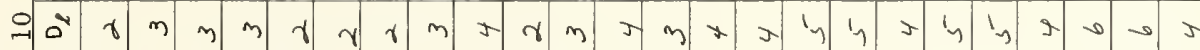

בn tr t J

若

产

ᄃ)

के

经厷

D h

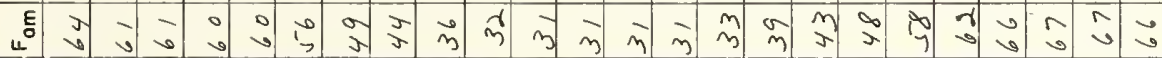

孷

톡

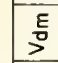

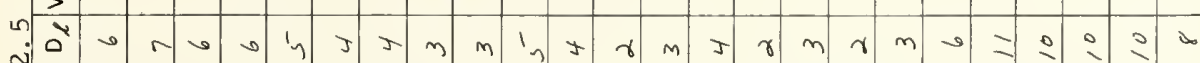

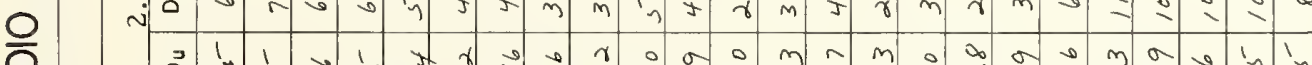

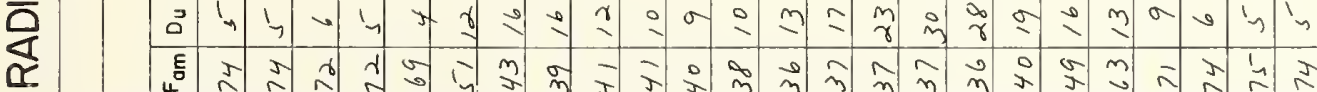

虫

舁

\begin{tabular}{c|c}
\hline \\
\hline
\end{tabular}

岂

$8>$

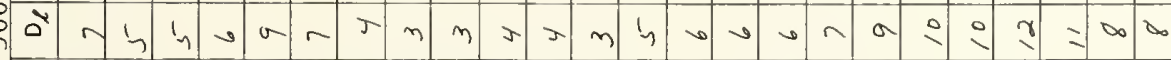

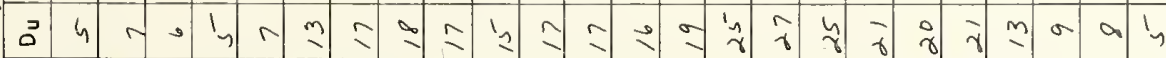

ह वे ₹

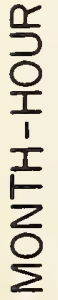

E्ञj

空

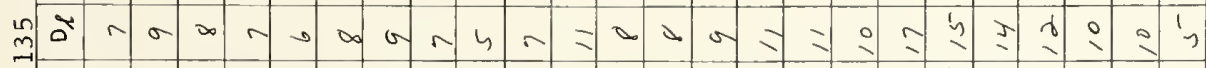

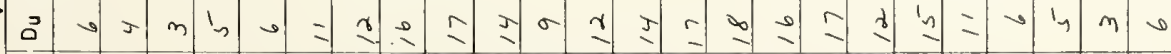

น

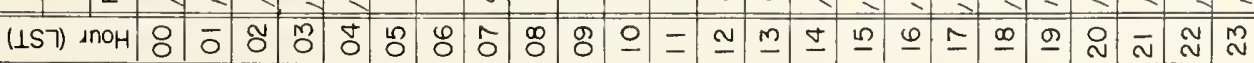




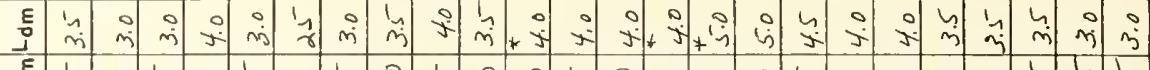

כ

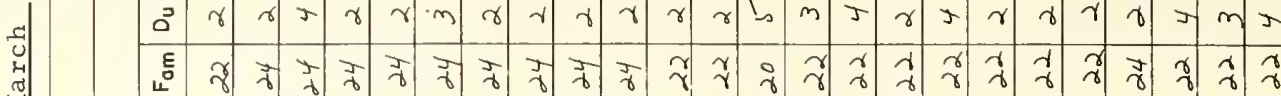

\section{E}

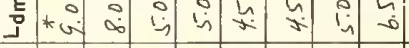

हE

음

a

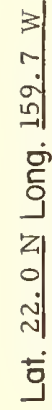

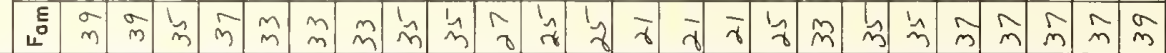

ง

$\bar{\sigma}$

E

$\stackrel{5}{>}$

in

○

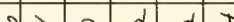

E

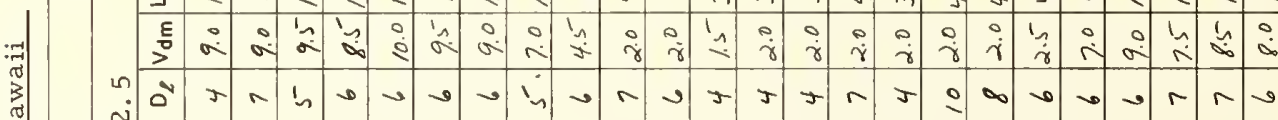

(3)

(1)

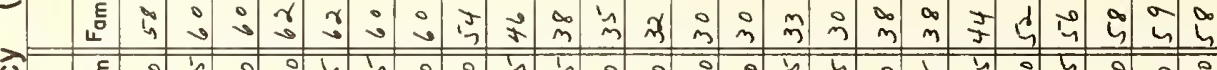

म

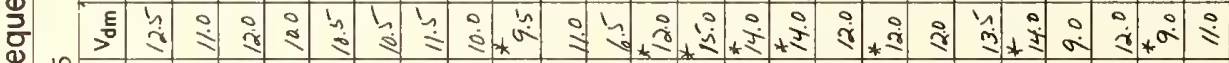
离

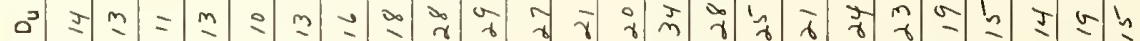

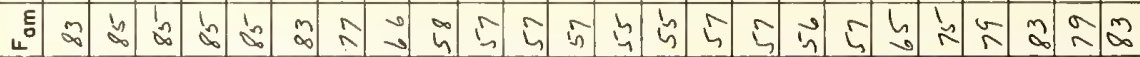

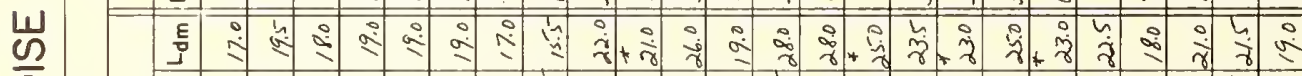

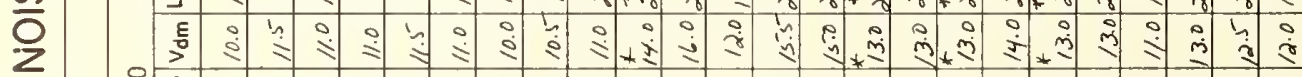

음 $\stackrel{0}{0}$

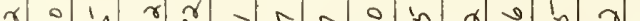

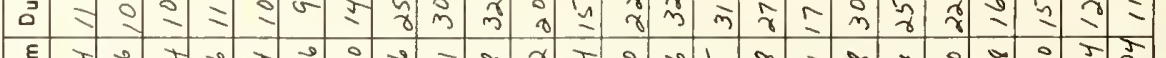

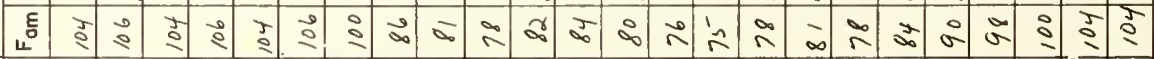
J 


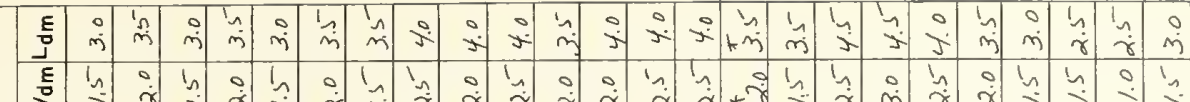

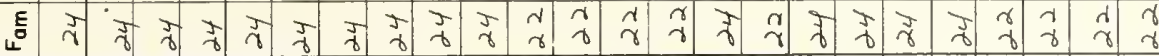

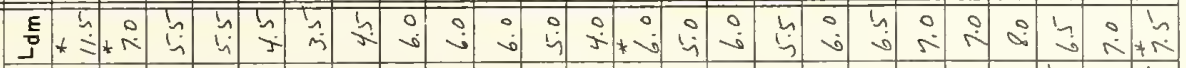

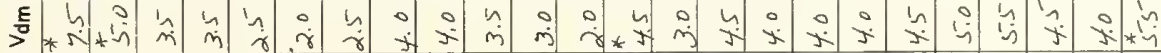

ㅇ.

3.

뭉

가

‥

ठํ

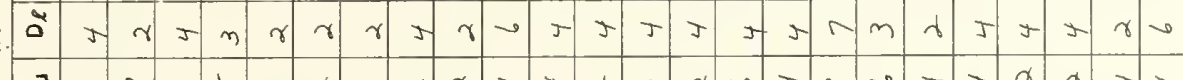

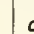

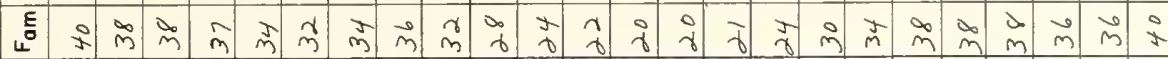

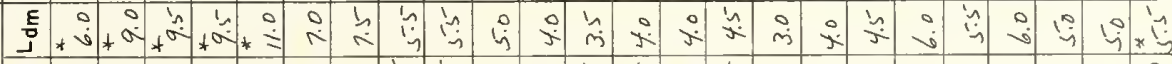

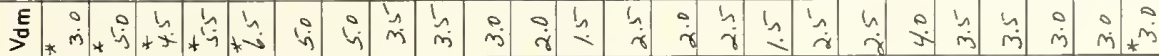

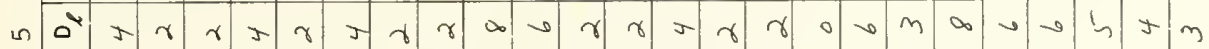

a

है की

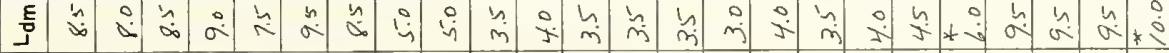

:

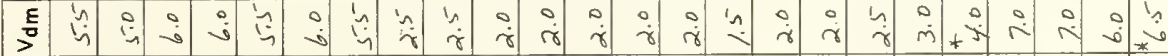
in

i

吾

उे

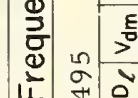

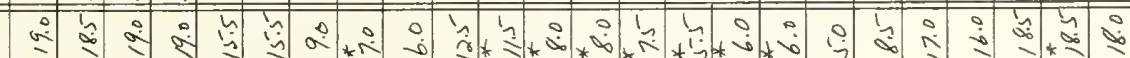

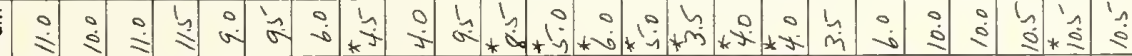

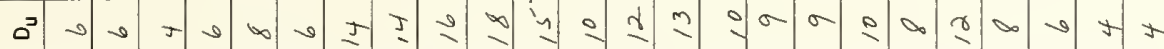

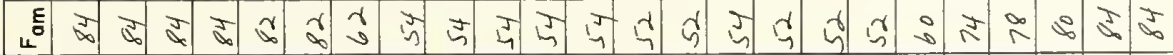

$\frac{w}{2}$

产 $\hat{N}$ iे

㫐

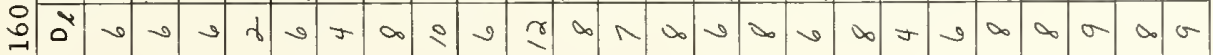

은

西

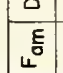

E

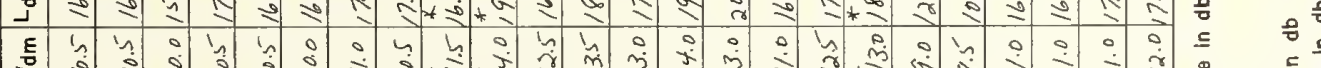

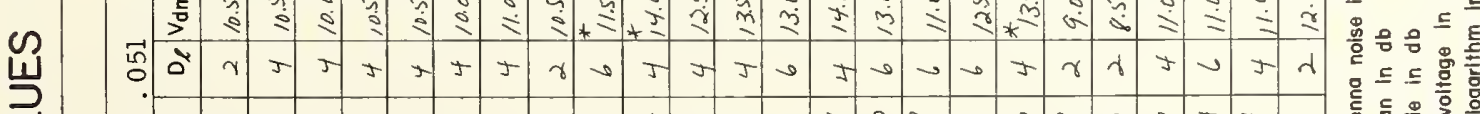

כ ว J

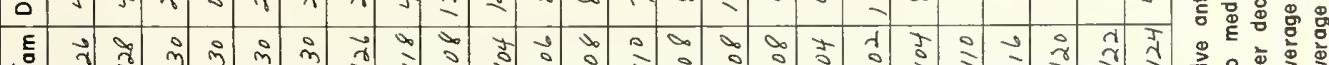

ह

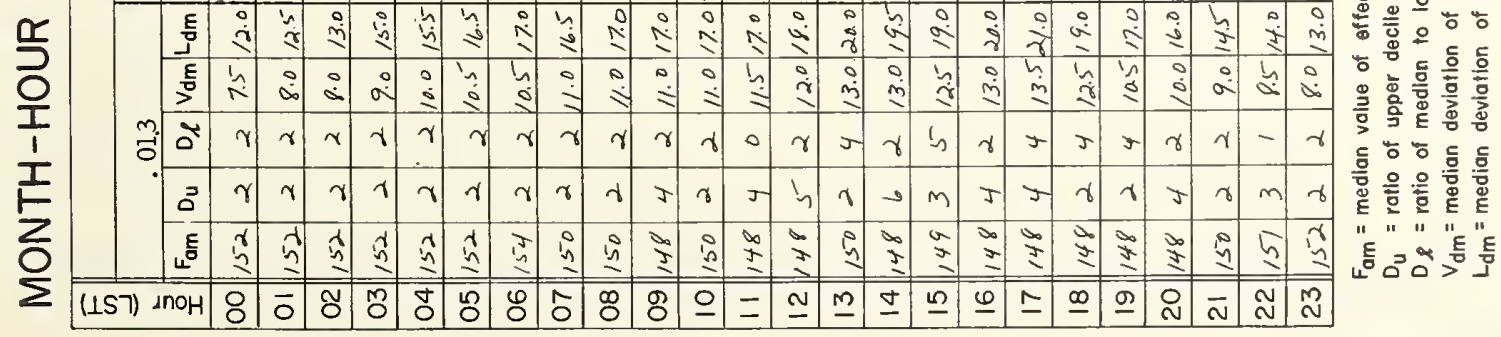




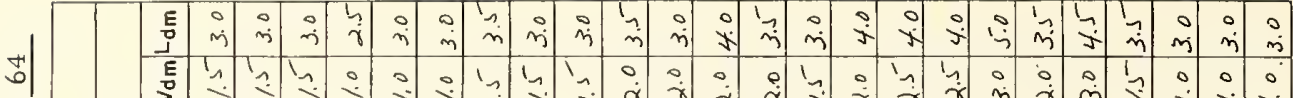

외

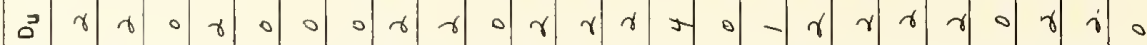

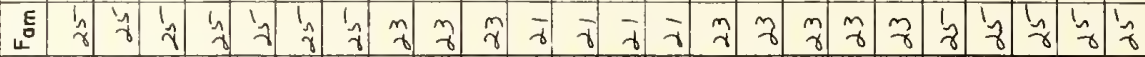

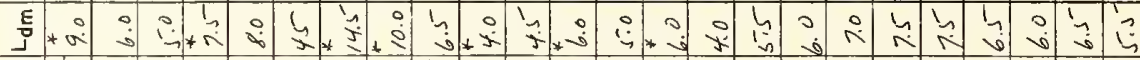
E

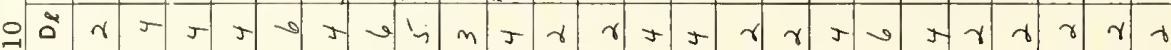

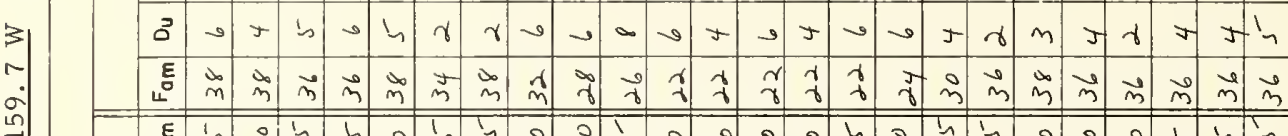

戛 $\hat{\imath}$ : E

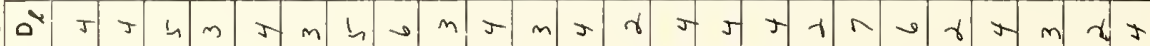
a

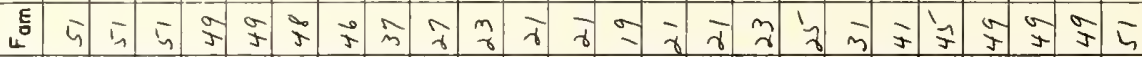

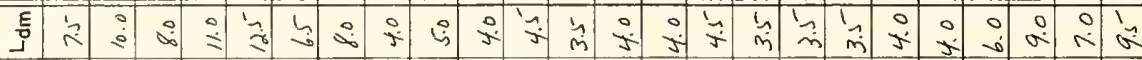

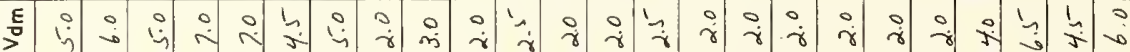

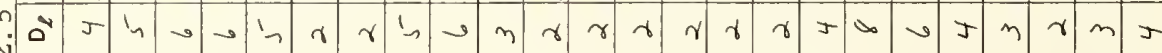

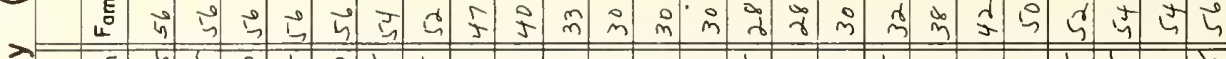

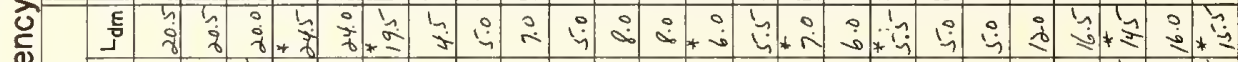
J E

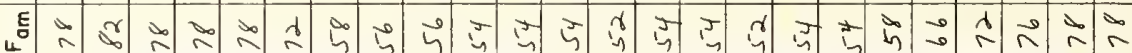

$\frac{1}{2}$

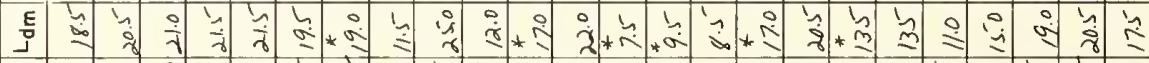

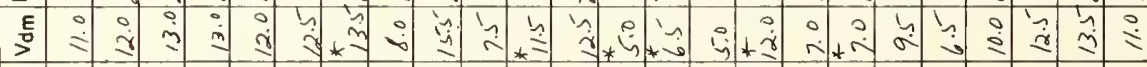
:

$\frac{0}{2}$

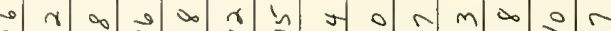

ह है ऊ E E

\1 เป็ ธี $\frac{m}{0} 0$ 


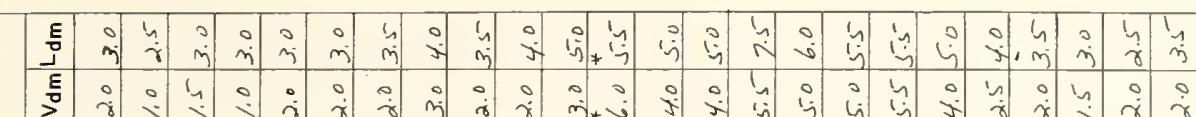

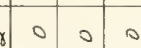

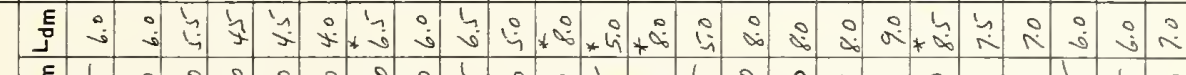
至 $\vec{y}$ में

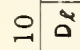

5

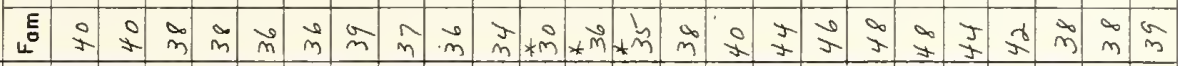

J

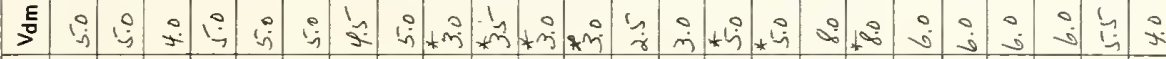
เ 0

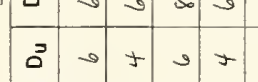

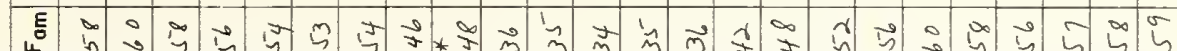

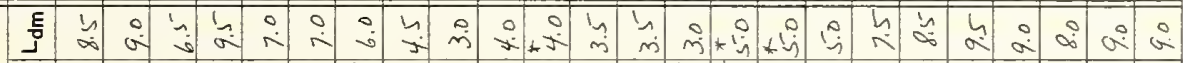

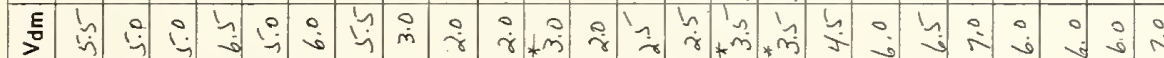

ก

i

$\stackrel{0}{\Xi}$

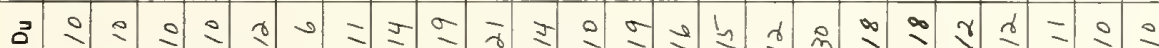

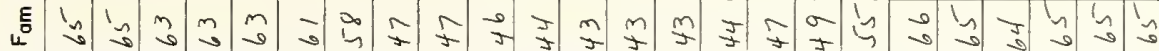

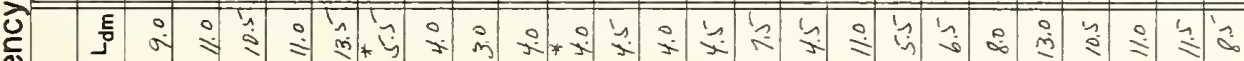

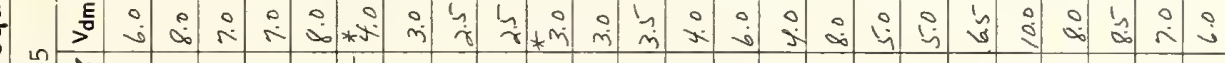
岁

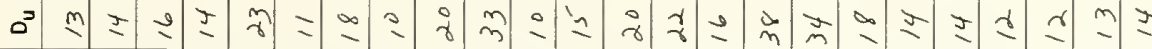

ए

$\frac{1}{2}$

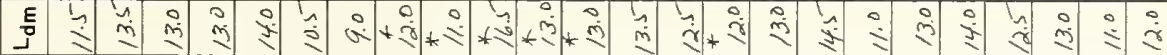

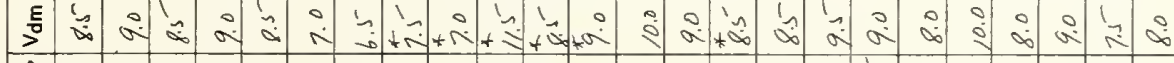
$\stackrel{0}{0}$

$\frac{0}{2}$

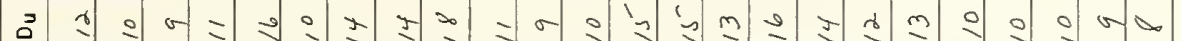

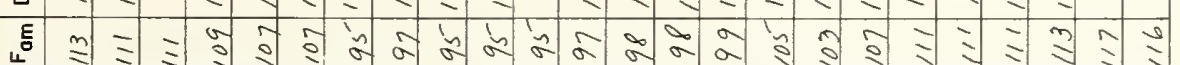

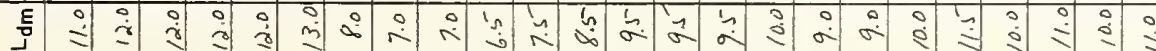

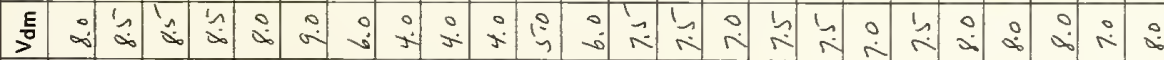
능 E

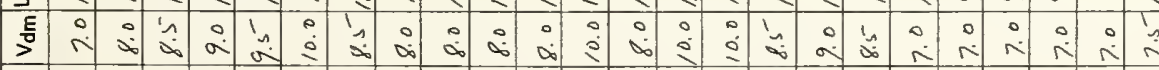
चै

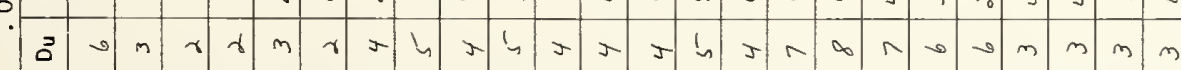

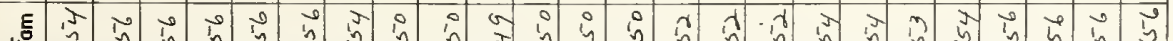




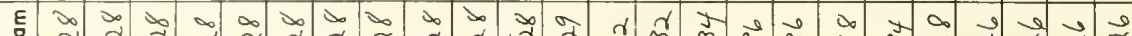
E o is o h h h o o h h h o h h

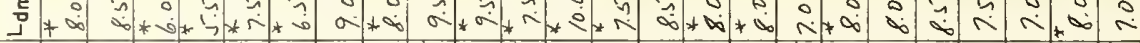

到

$0^{3}$

乙

$\stackrel{\infty}{\infty}$

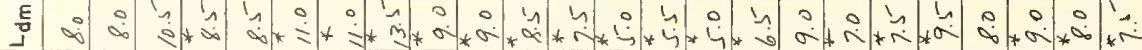
हू⿻

$0^{3}$

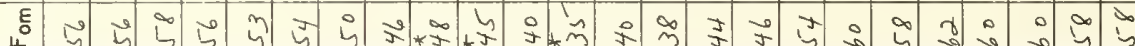

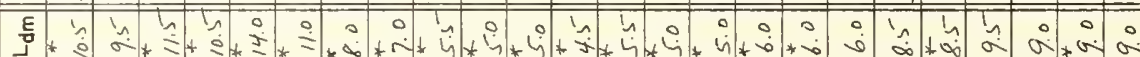

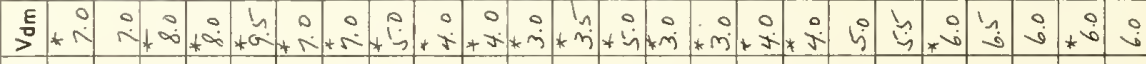

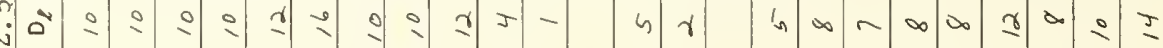

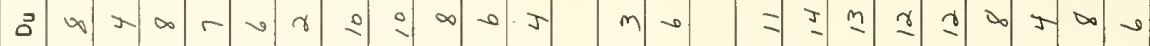

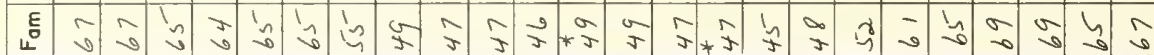

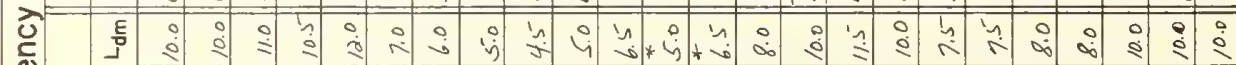

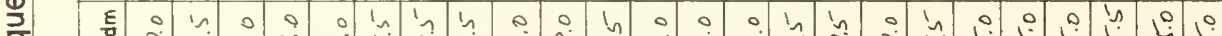
O $>0$ 杀苛

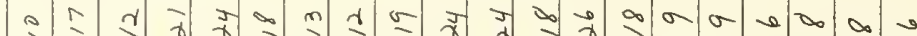

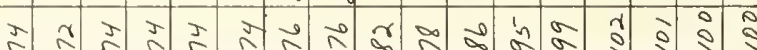
น.

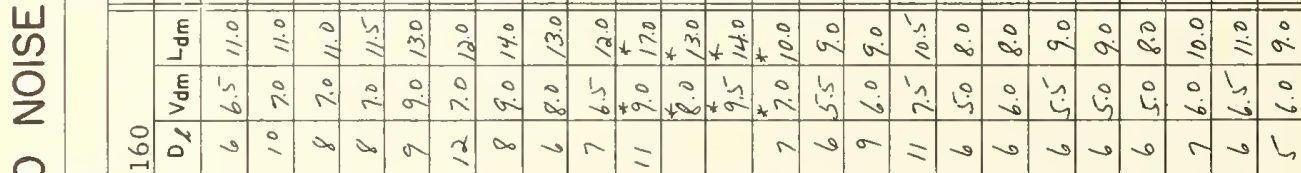
$\frac{1}{0}$ a

$\frac{1}{\alpha}$

虫

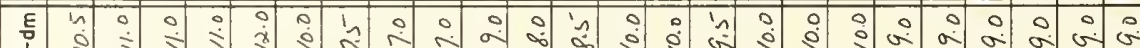

Щ

E เกี ठิ

\section{E \\ a}

ב

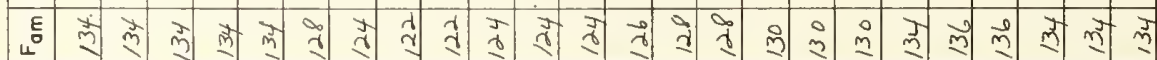

है

$\frac{1}{5}$
$\frac{1}{1}$
$\frac{1}{5}$
$\frac{1}{2}$

हᄐ ?.

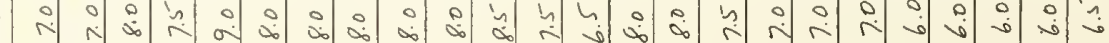




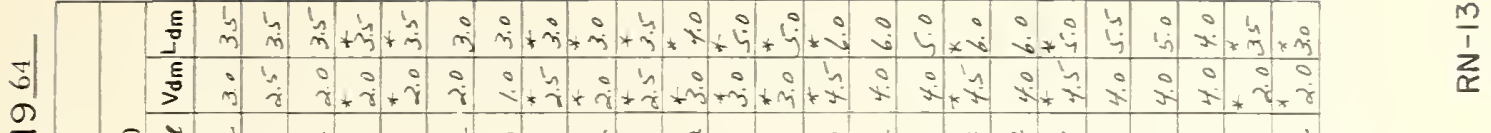

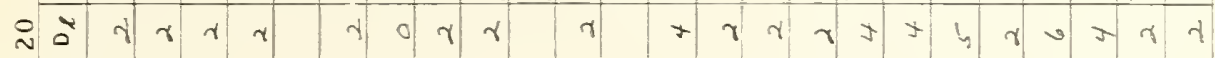

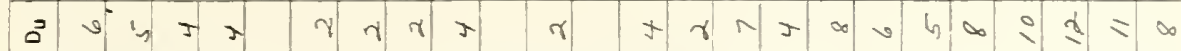

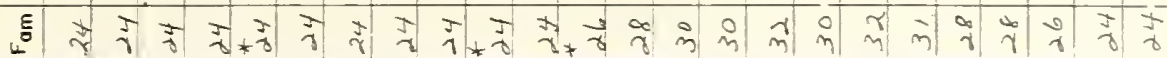

* E

*

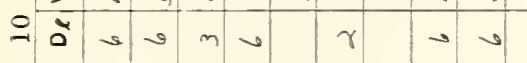

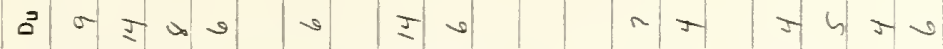

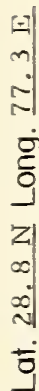

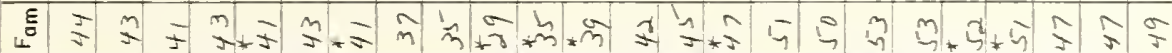

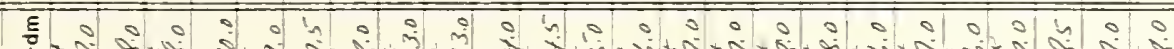

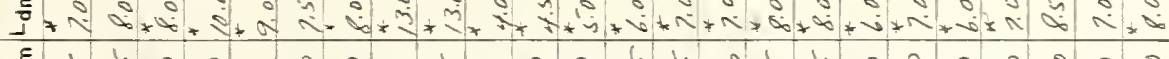

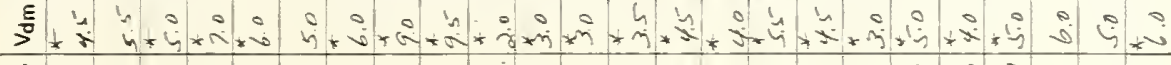
0

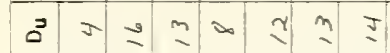

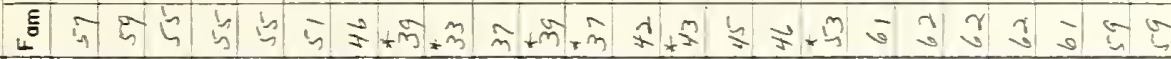

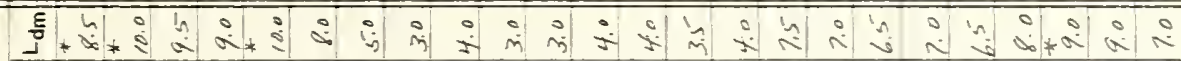

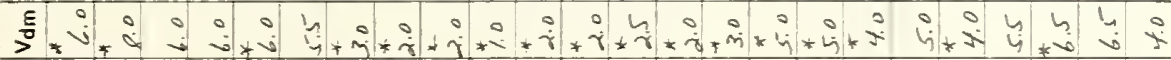

is $\rightarrow$ it

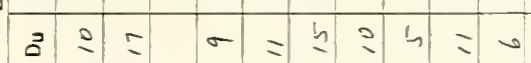

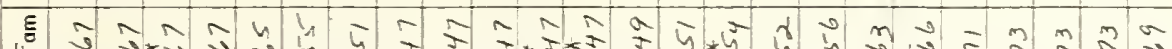
=

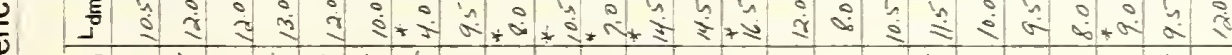

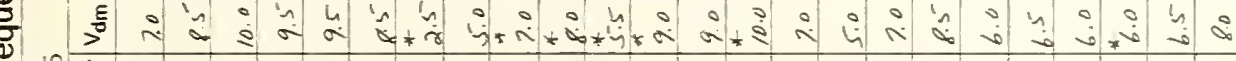

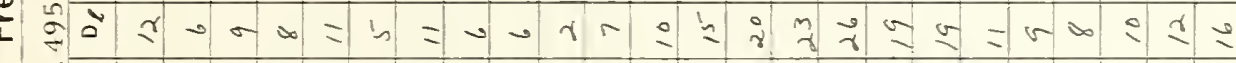

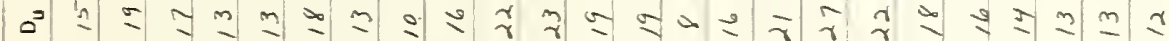

एक

$\frac{w}{2}$

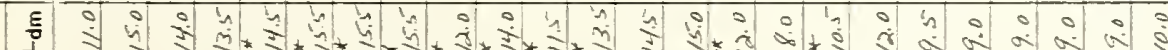

ह

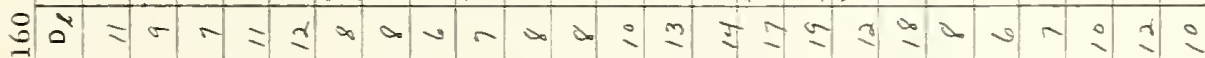

J $=$ y.

$\frac{0}{2}$

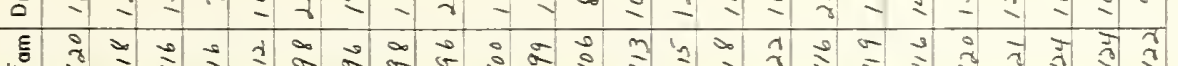

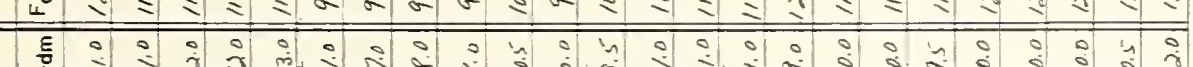

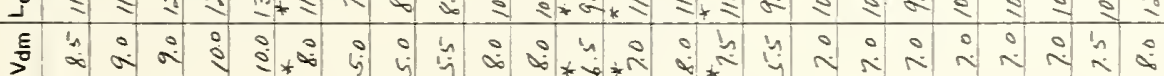

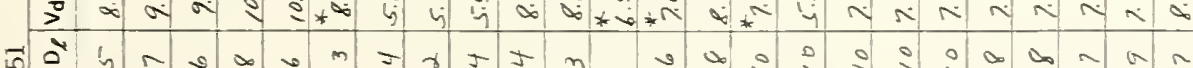

Щ

ב.

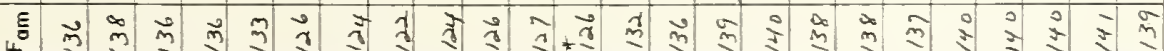

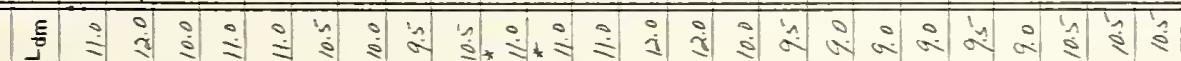

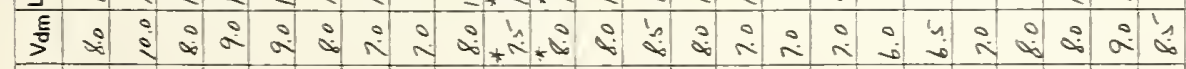

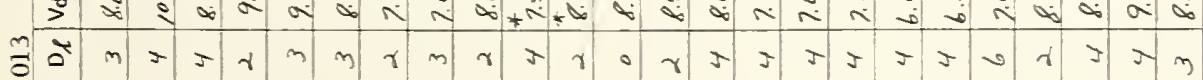

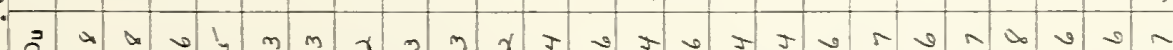

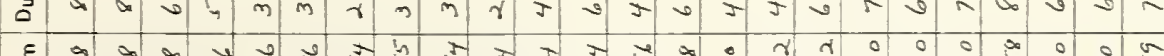

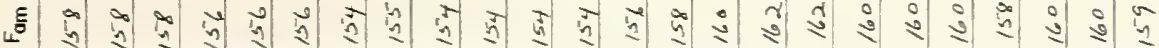

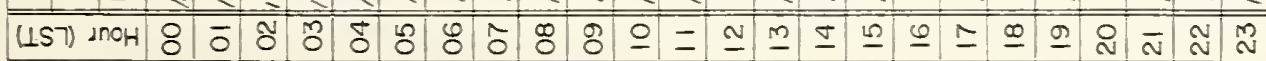




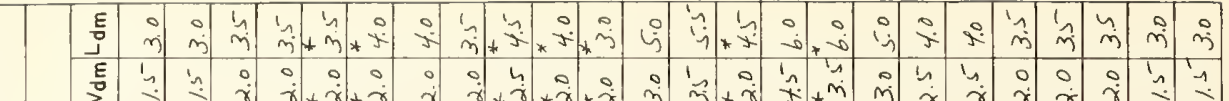

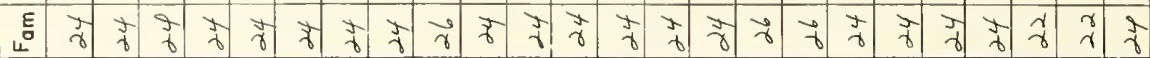

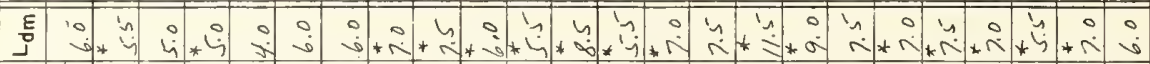

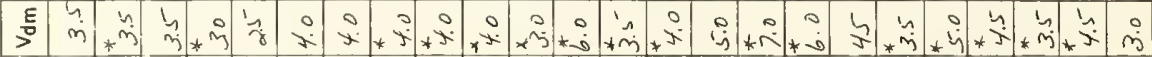
임

뙤

3

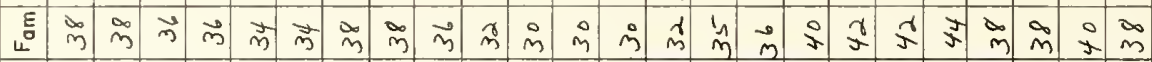

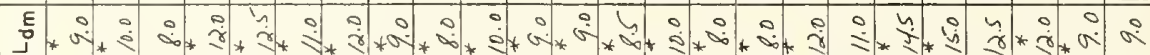

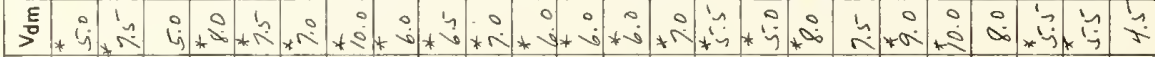
$n a^{y}+$ J a $r \rightarrow$ a

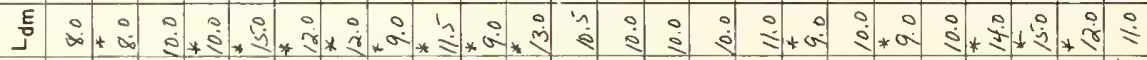

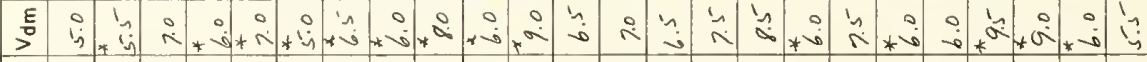

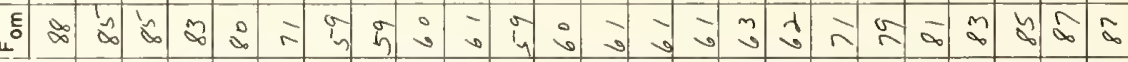

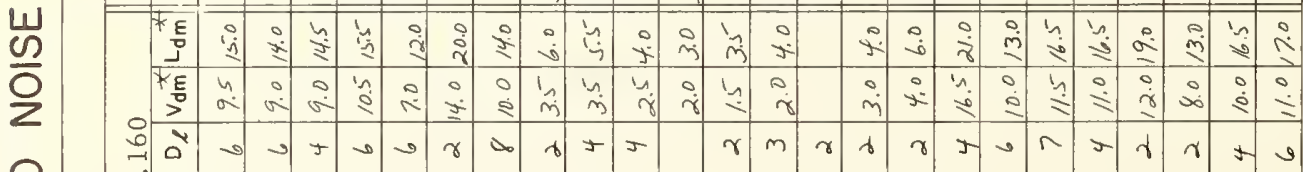

음

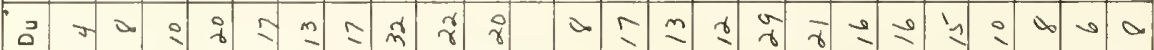

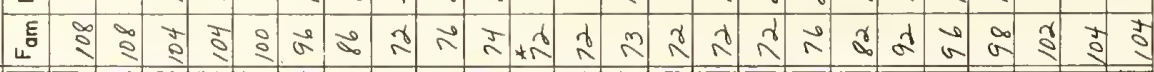

है

岁

岂

E

जी

(3)

a

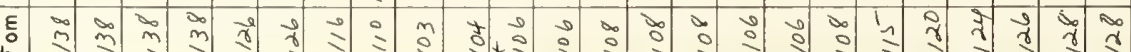

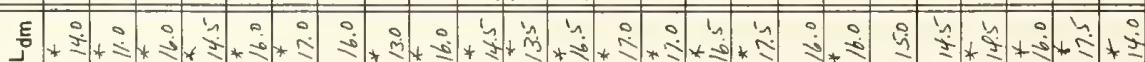

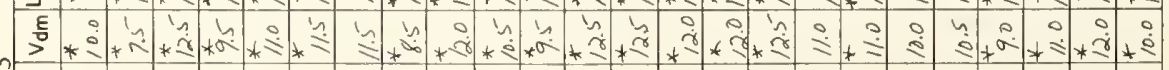

हैة

产

ב

${ }^{E}{ }^{\circ}$

(IS) גกoH 


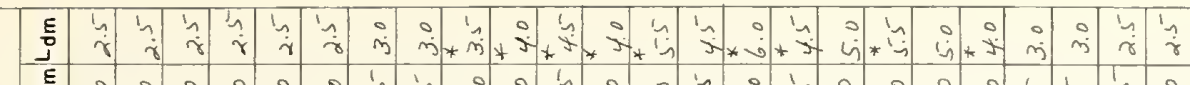

$\circ$ $\stackrel{\bullet}{\vee}$

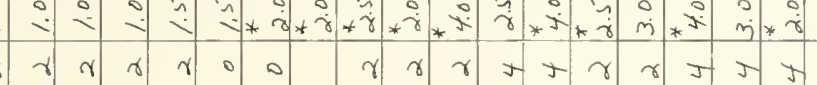

|

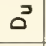

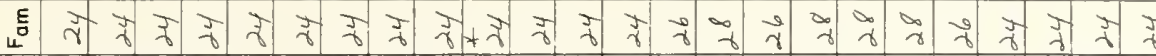

E

in

E

旁

हE ก

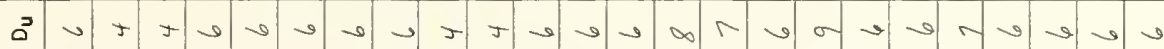
है

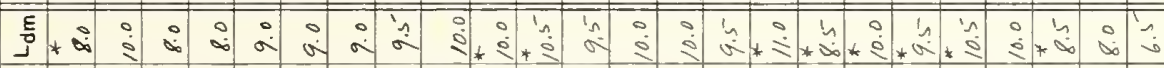

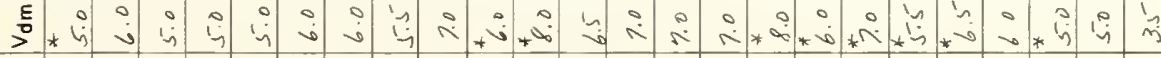
वี

in

$\sum_{\Xi}^{0}$

ב

E

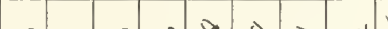

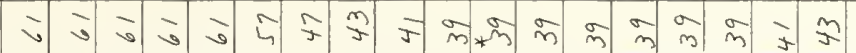

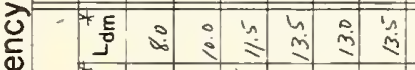

ㄷำ

政

的

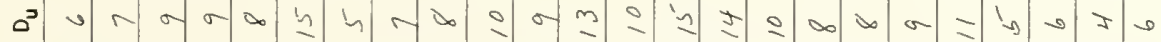

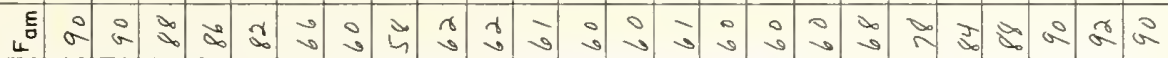

$\frac{w}{2}$

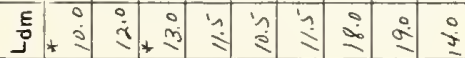

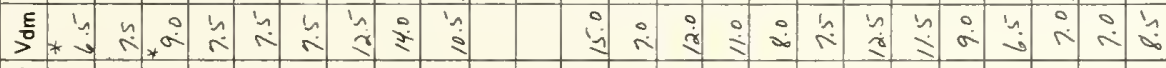

$\stackrel{0}{0}$

$\frac{0}{2}$ (

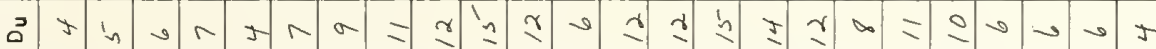

L

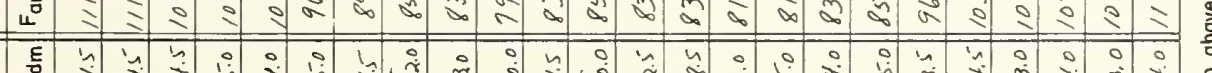

$\stackrel{4}{0}$

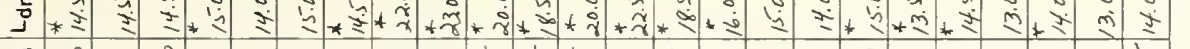

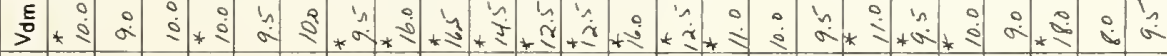

山

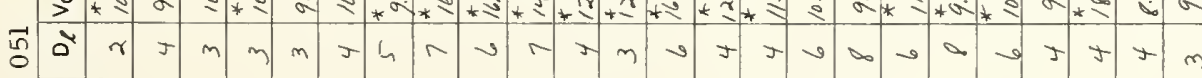

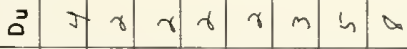

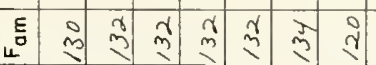

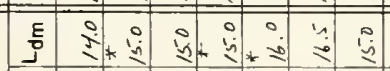

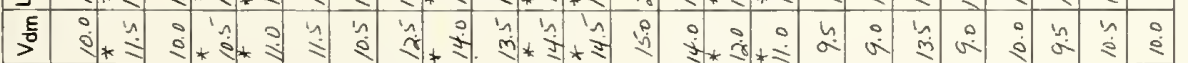

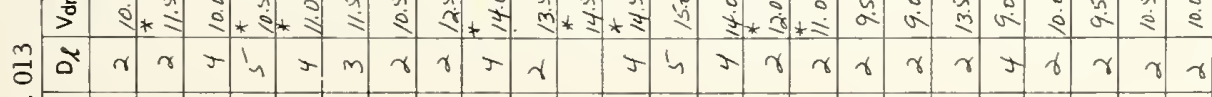

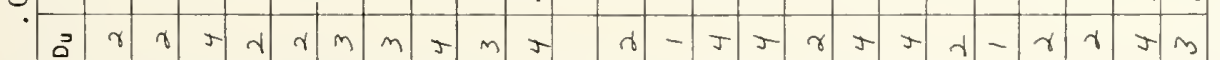

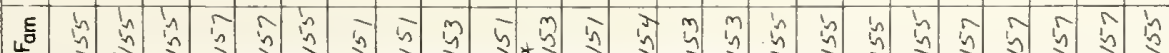

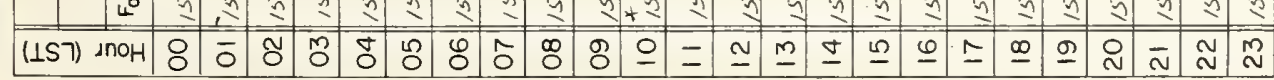

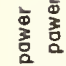

$\therefore \quad 5$

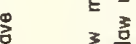

흥 융 응 움용 도

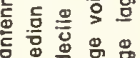
突워

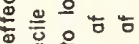
t 㐫

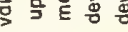
동ㅎㅎ듬듬

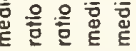
" 


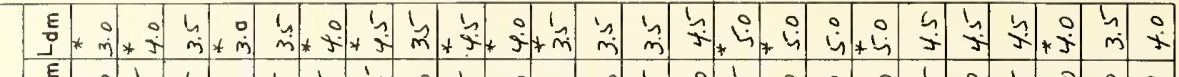

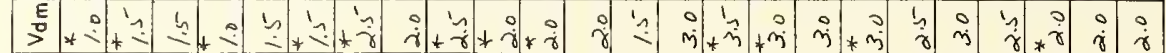

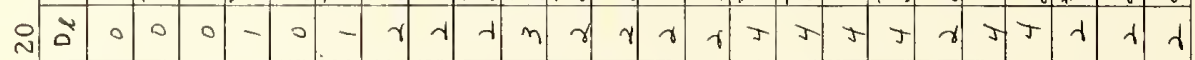

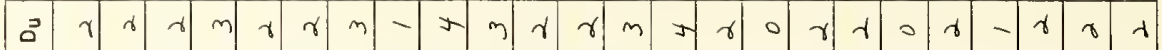

E

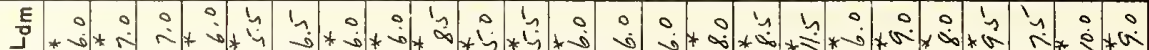

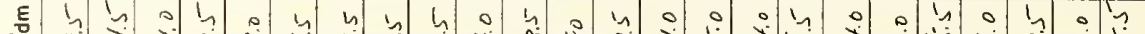

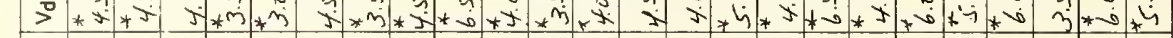

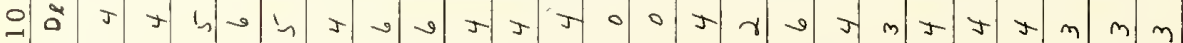

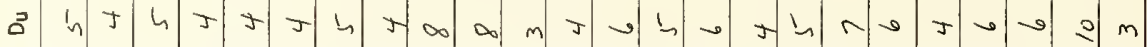

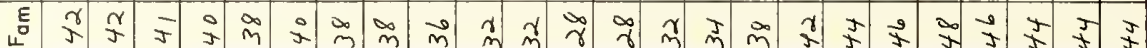

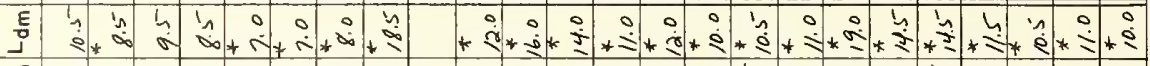

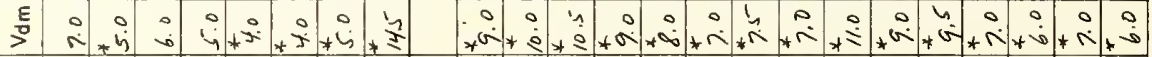

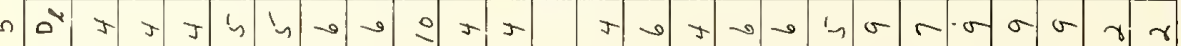

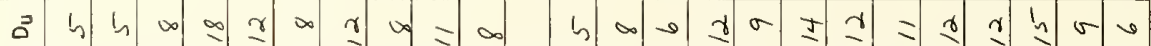

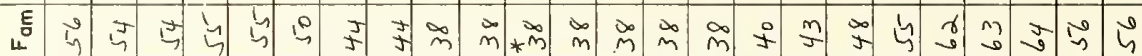

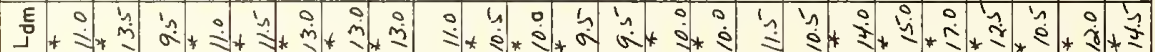

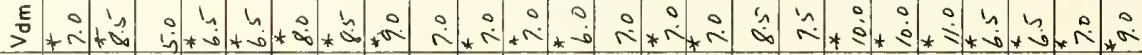
동ำ in i.

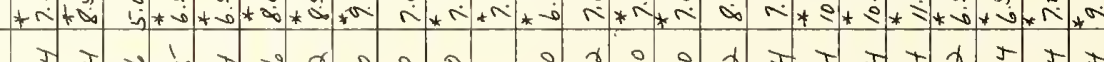

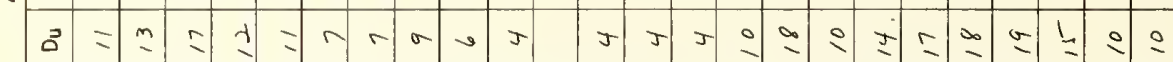
ह

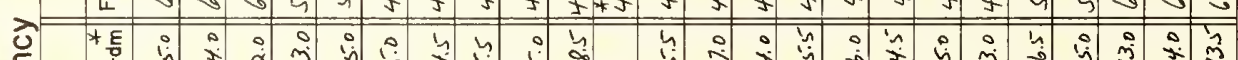

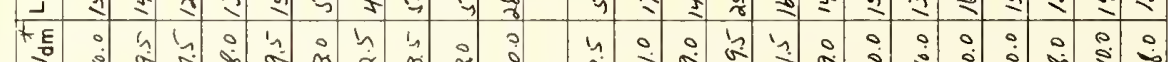

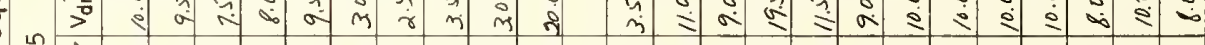
等方

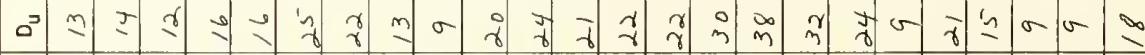

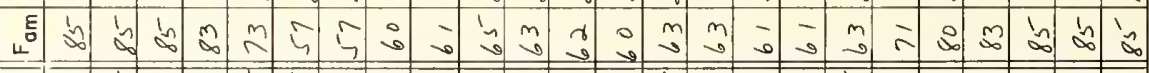

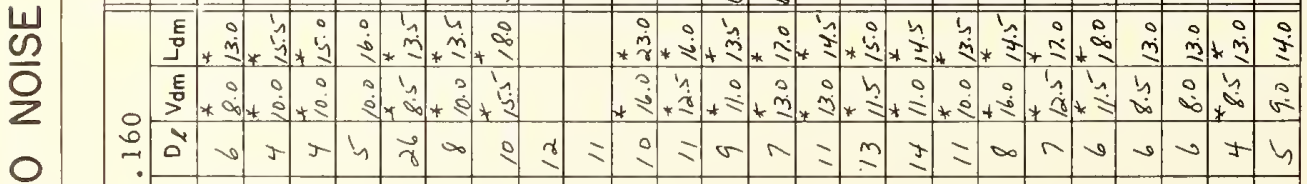

$\frac{0}{\frac{1}{\alpha}}$

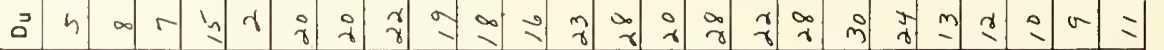
ह है

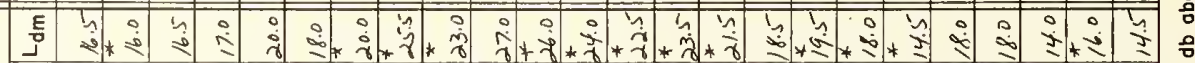

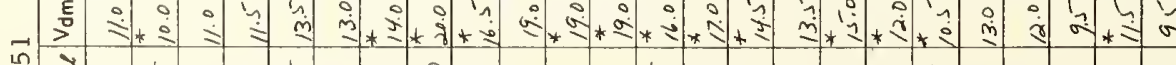

\section{崖} เก

ว

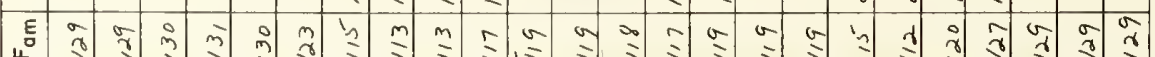

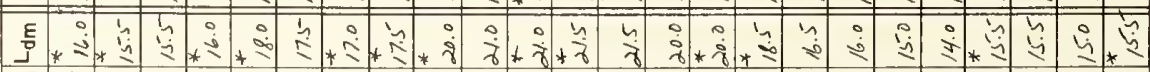

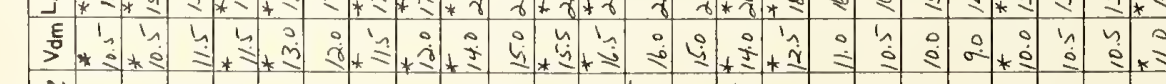

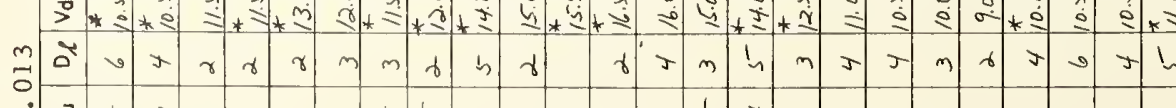

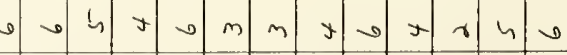

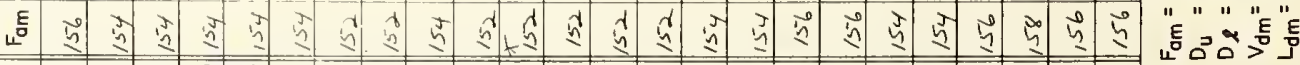

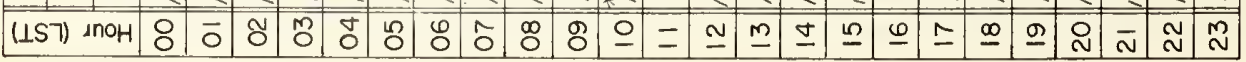


मै

\begin{tabular}{|c|}
\hline$\underline{0}$ \\
\hline \\
\hline$\xi$ \\
\hline
\end{tabular}

일.

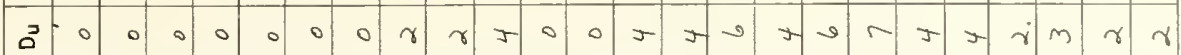

है

E

旁

ह

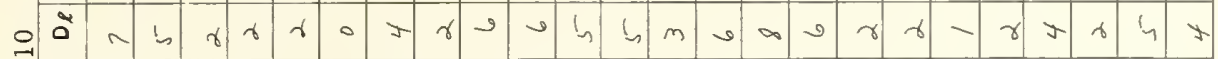

a

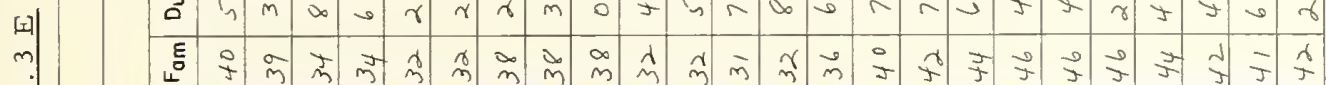

$\infty$

뭄

E

E

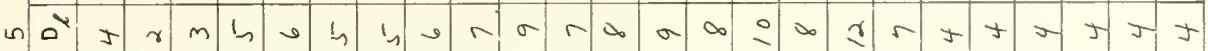

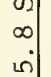

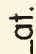

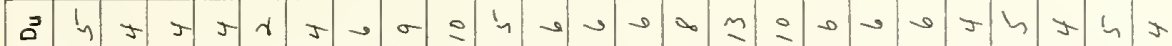

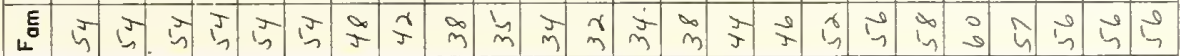

先

E⿱一𫝀丁

E

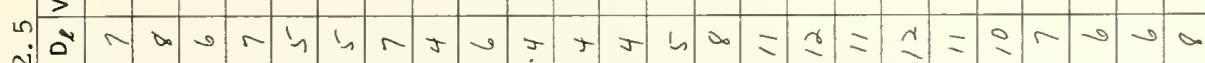

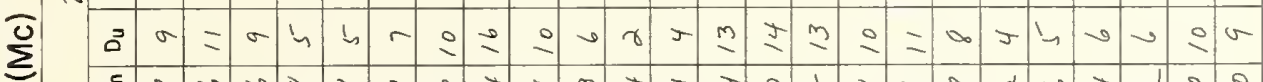

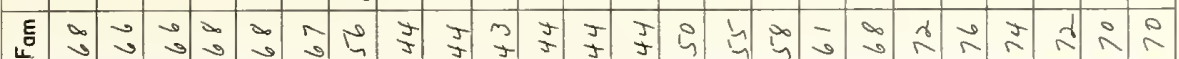

ठิ

을

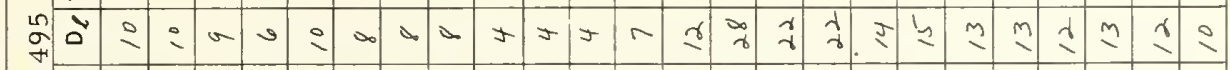

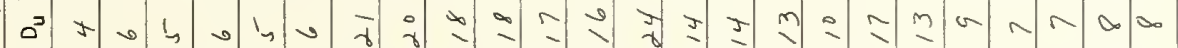
फ ह न

$\frac{4}{2}$

E

$\frac{E}{>}$

:

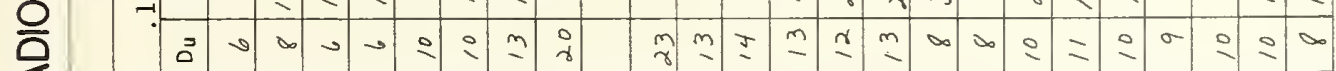

준

\begin{aligned}$\Psi^{\circ} \\$\hline \hline\end{aligned}

岁

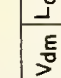

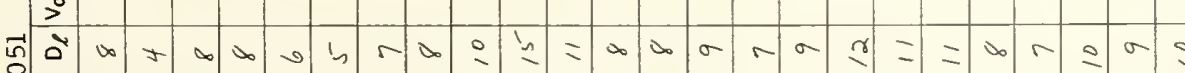

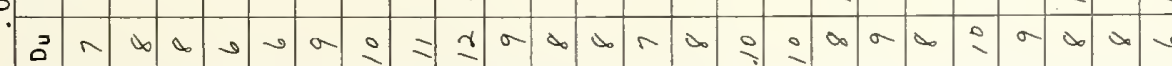

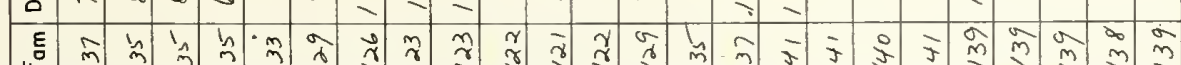

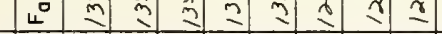

$\frac{1}{3}$
$\frac{1}{1}$
$\frac{1}{E}$
$\frac{1}{2}$

?ै

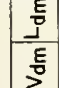

$\stackrel{2}{0}$

1

ह

जो हैं ज्ञ

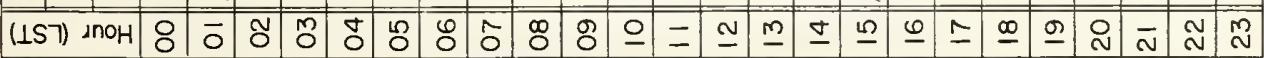


פַ)

ㅇํㅇ

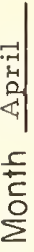

3

点

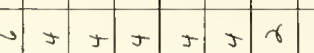

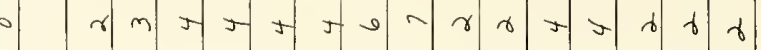

$\frac{5}{5}$

톡

E

에

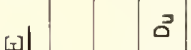

m.

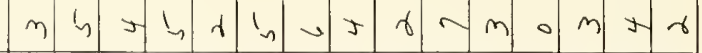

|

ธ்

E

$\stackrel{5}{\mathcal{E}}$

in $0^{4}$

$\infty$

ก!

节

ב

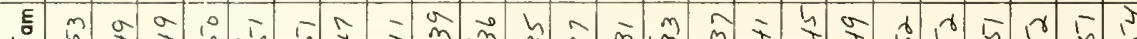

兽|

E

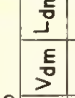

in

芩

.า.

c.

난.

幺

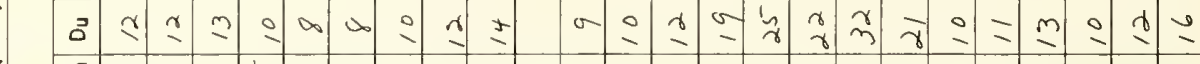

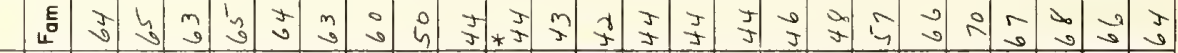

至

(1)

$\stackrel{2}{2} \frac{2}{2}$

(

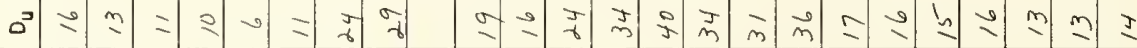

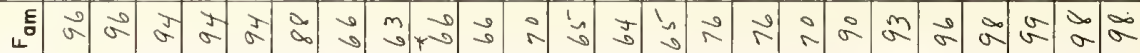

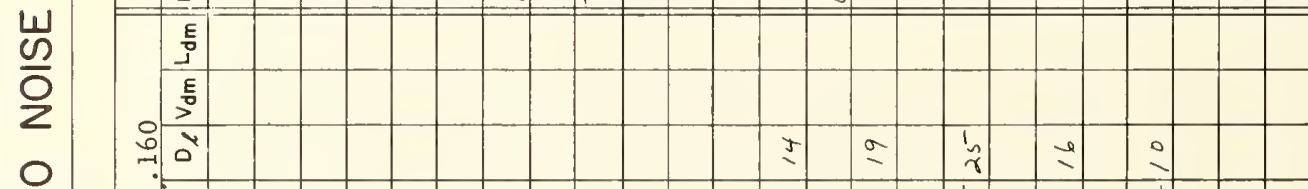

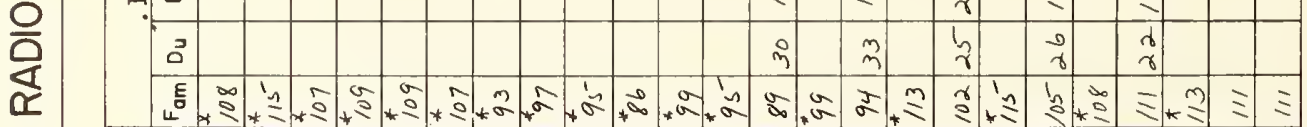

岁

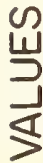

(E)

E

เู่

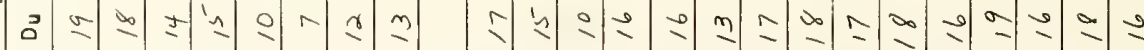

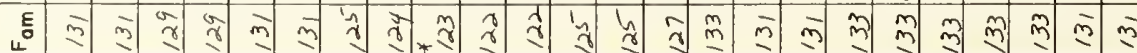

E

ㅁ
O
$\frac{1}{1}$
I
$\frac{1}{0}$

등

$\stackrel{5}{2}$

(5)

वี

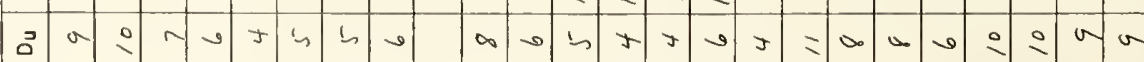

E

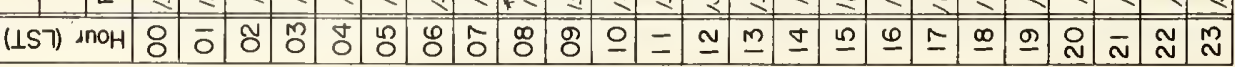


0

$\int$

$1 \mid \frac{1}{4}$

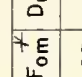

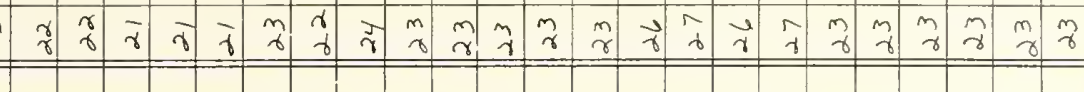

$\stackrel{\text { c }}{\stackrel{c}{c}}$

름

E

응

LI 5

?

ำ

$\infty$

i

+ே.

包

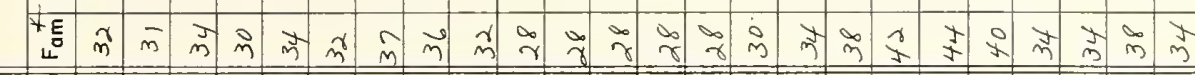

(j)

E

E

เก

o

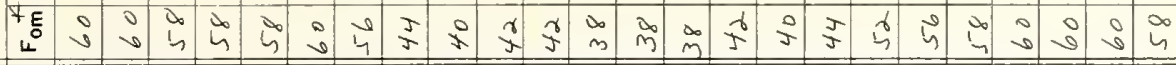

통

in

$\frac{E}{2}$

¿ 3

* है

$+$

ठิ

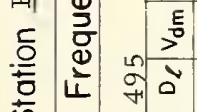

क

$=$

*E

$\frac{u}{2}$

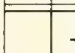

E⿱一𫝀口)

:

은 $\rightarrow$

진

낭

$\frac{1}{2}$

$\frac{1}{2}$
$\frac{1}{1}$
$\frac{1}{1}$
$\frac{1}{2}$

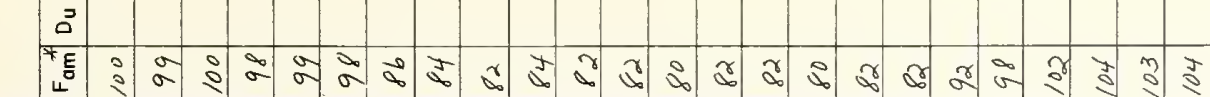

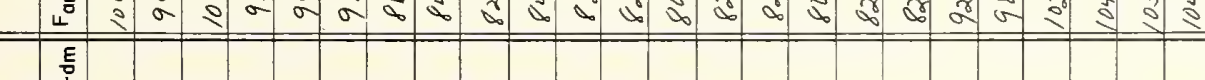

통

जี ธे

a

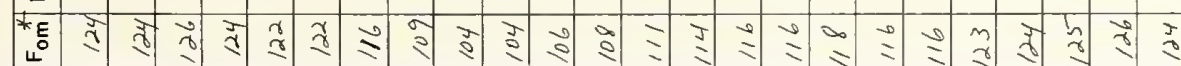

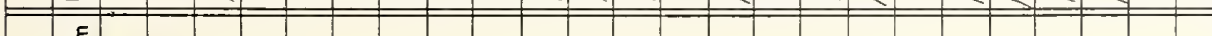

西

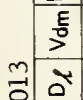

$\stackrel{m}{0}:$

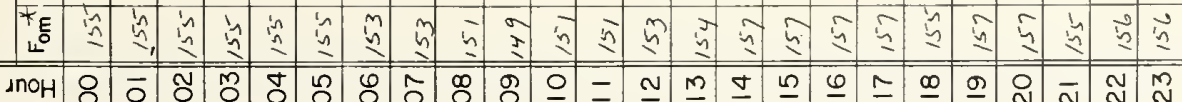

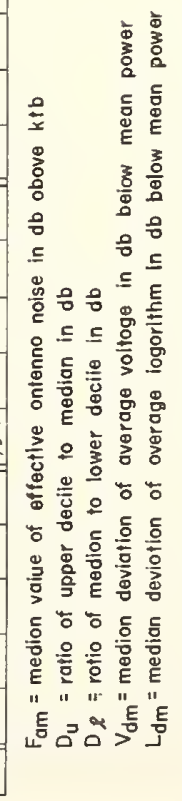




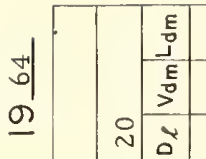

a

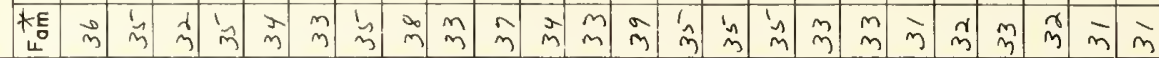

E

旁

틍

$\stackrel{-10}{0}$

3

$\infty$

ণ্

公

$m$

تே.

(

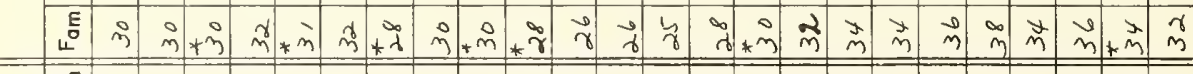

E

$>$

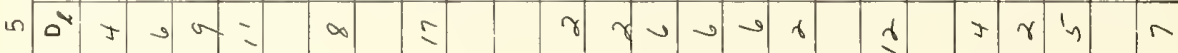

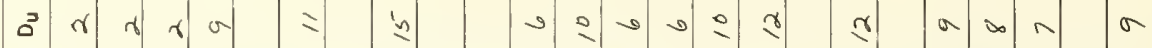

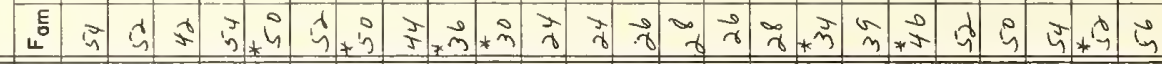

E

0
0
0
0
0
0
0
0
0

E

in

J

들

它

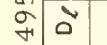

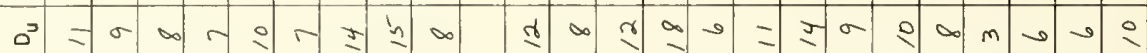

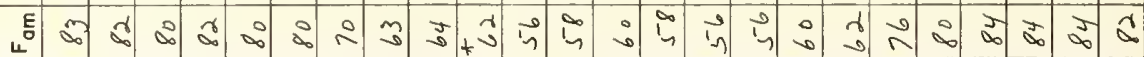

峞

E

틍

$8>$

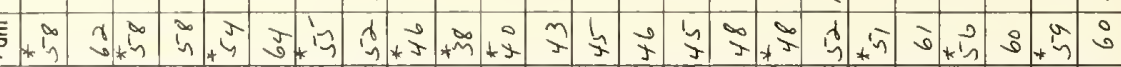

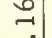

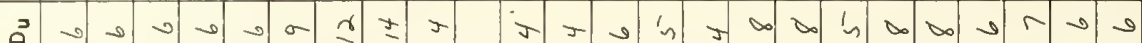

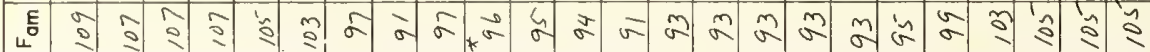

㝵

亗

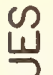

E

in

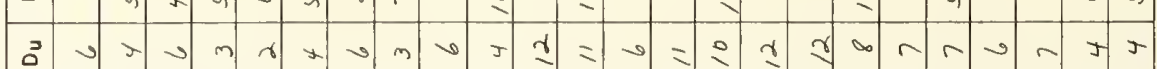

ह

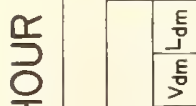

$\stackrel{m}{0}$

$y$

马

若

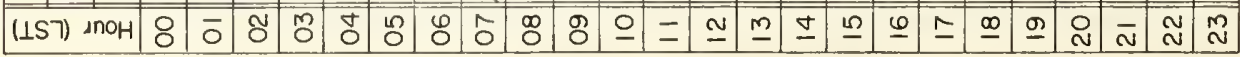

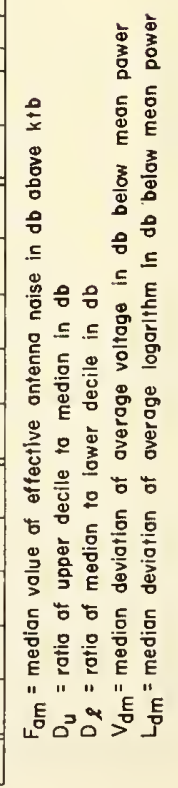


욜 म

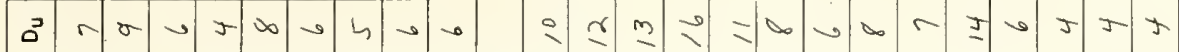
ह

돔

E

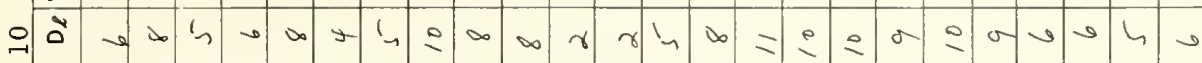

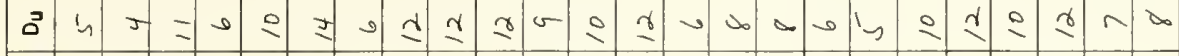

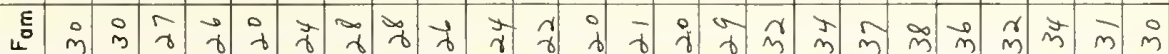
है

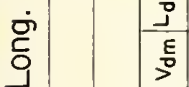

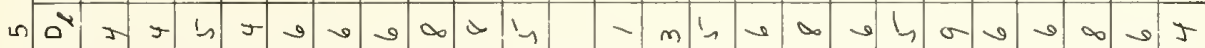

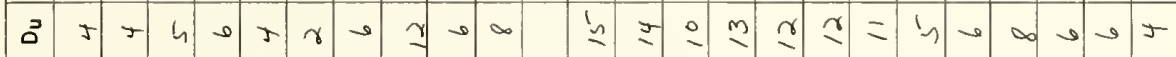
है 可

E

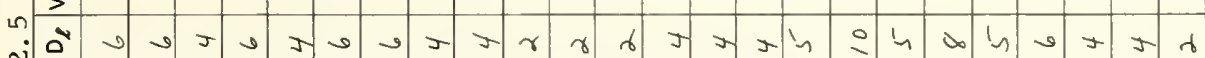

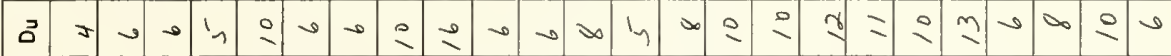

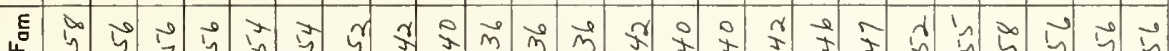
4 .

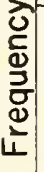

독

हE

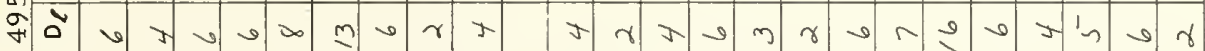

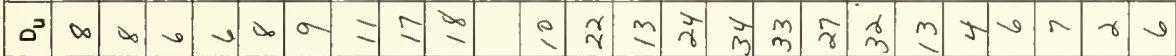

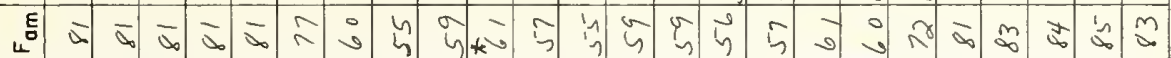

$\frac{1}{2}$

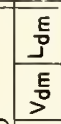

용

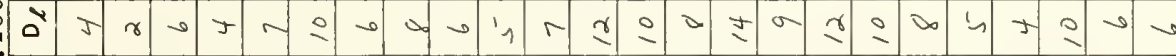

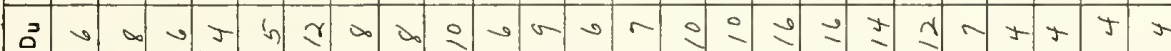

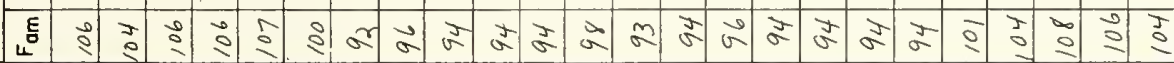

통

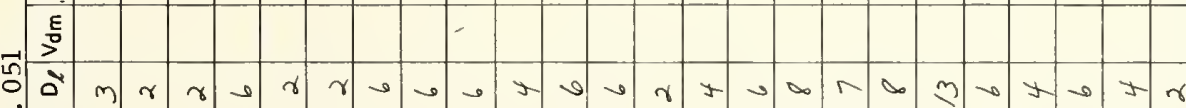

$\stackrel{2}{2}$ z 苋

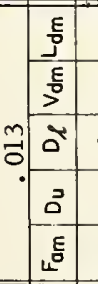

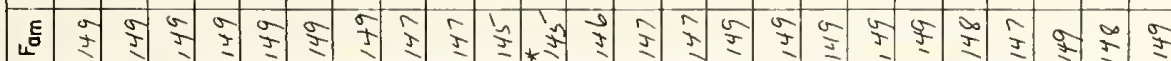

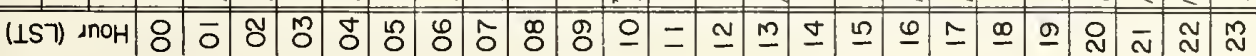




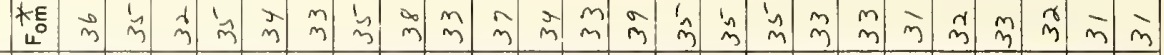

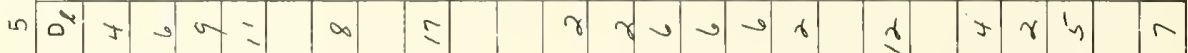
s.4ra

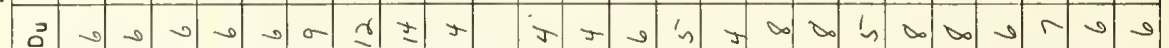

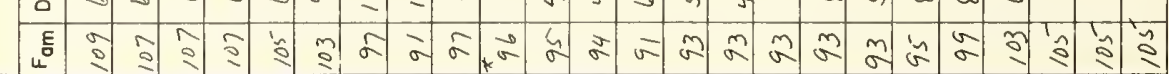

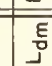

亗

जी

岂

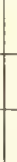
E

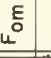

\section{$\frac{\mathrm{E}}{\mathrm{g}}$}

高

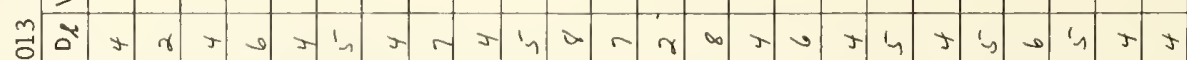

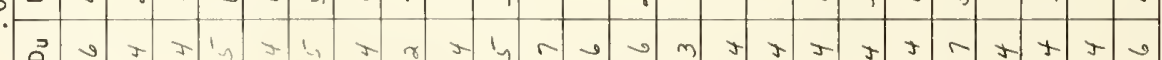

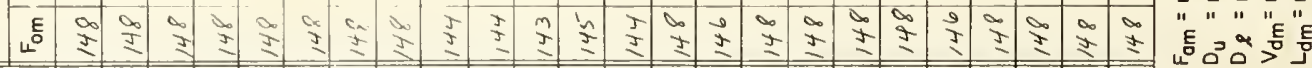
(IS7) มกH 


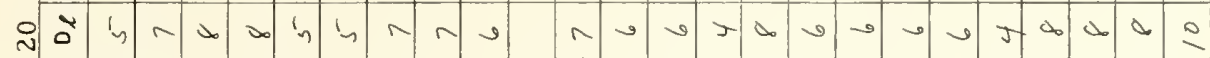

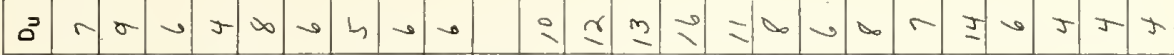

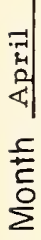

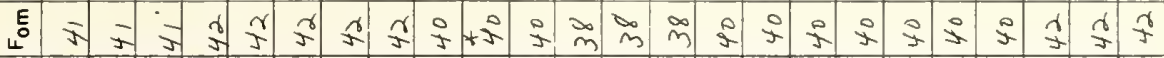

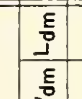

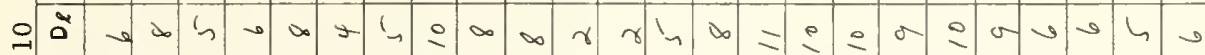

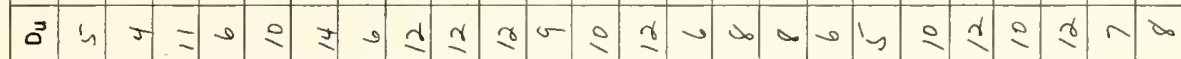

3

E

ह

宅

$\stackrel{E}{>}$

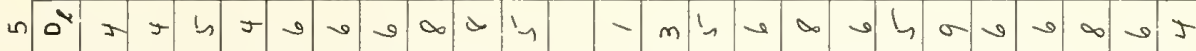

张

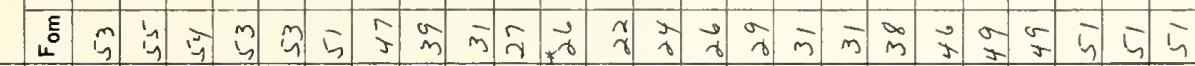

ఫ)

:

E

है

in $>$

包

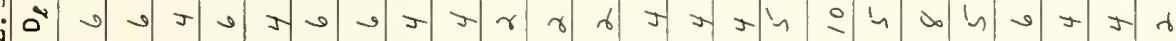

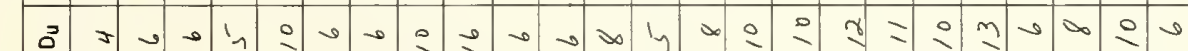

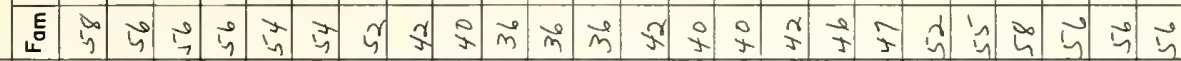

仓ิ

索

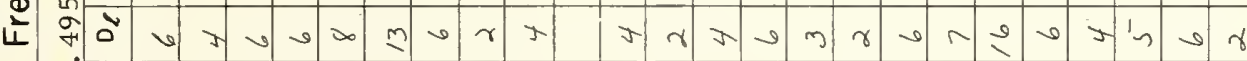

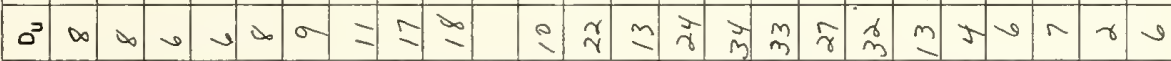

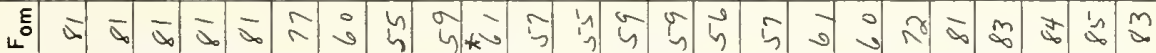

宸 4

E

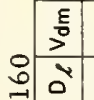

3

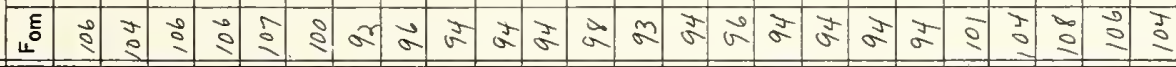

है

岂

$\rightarrow$

चु

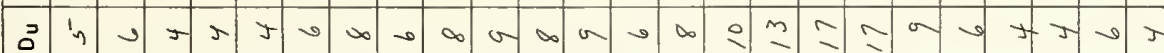

ह

$\vec{s}$

疍

$\frac{1}{3}$

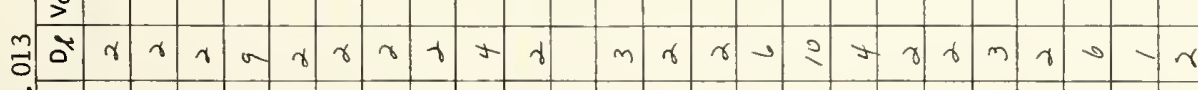

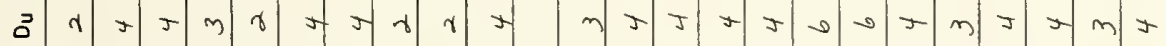

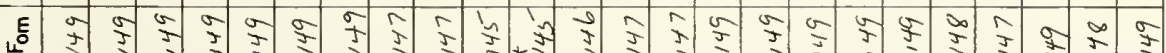
(LS7) มnoH 8 o 


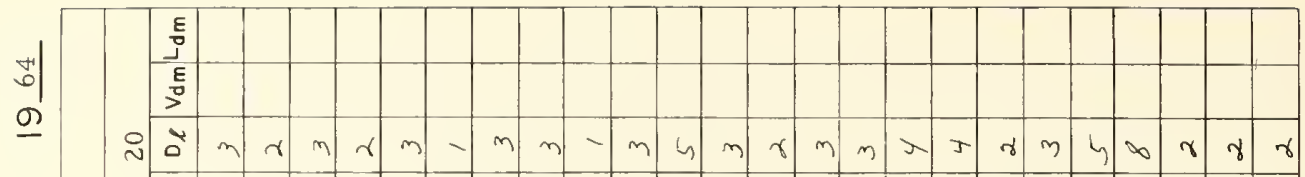

ว

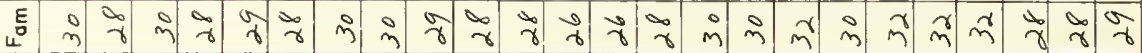

(1)

톡

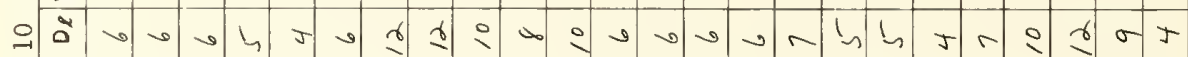

$$
\text { है }
$$

经年

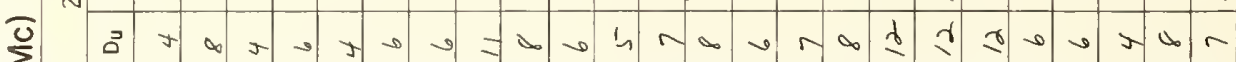

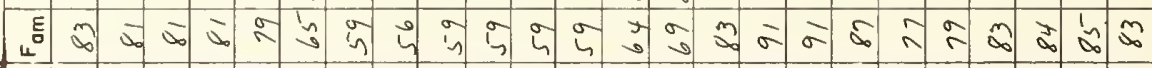

$\frac{1}{\infty}$
$\frac{0}{0}$
$\frac{0}{2}$
$\frac{1}{\alpha}$

$\frac{E}{\partial}$

E⿱

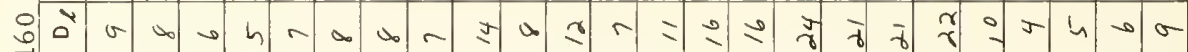

כ

ह

岁

岳

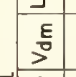

岂

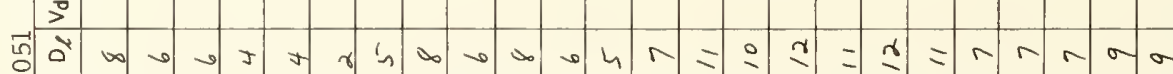

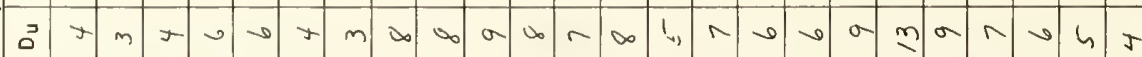

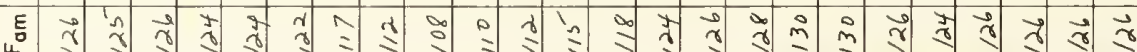




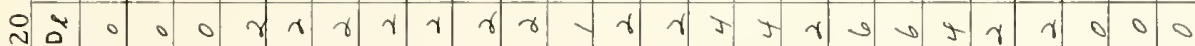

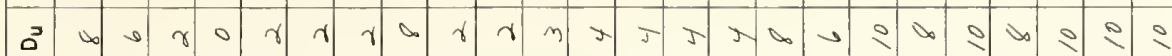
Е 톰

E

웅 ב

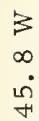

ஜ்

E

官

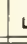

焉

E

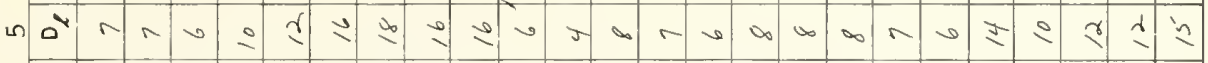

m.

+ே.

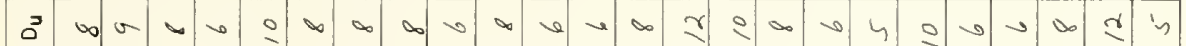

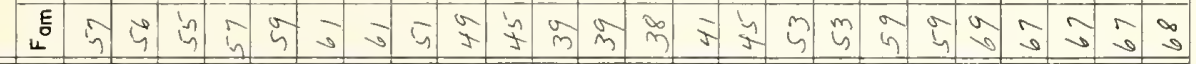

年

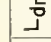

เก $>$

$\stackrel{\Xi}{\Xi}$

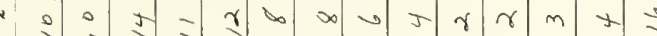

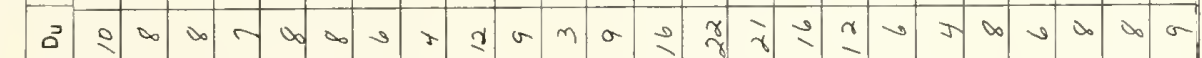

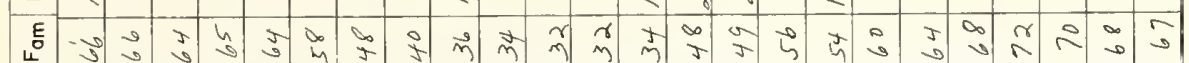

त्रे

당

$\frac{-0}{5}$

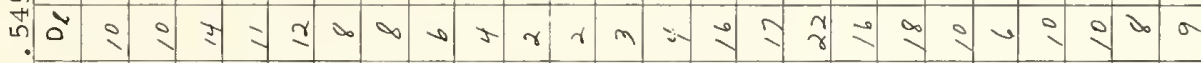

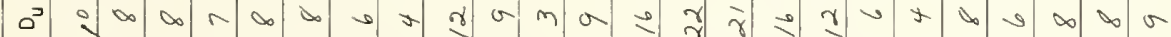
एह

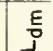

焉

虫

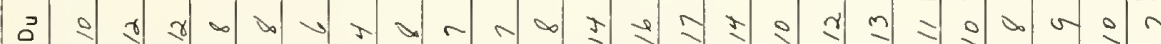
ह है : : :

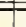

E

E

荘

m

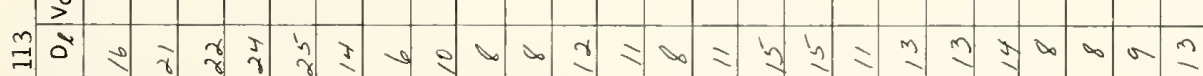

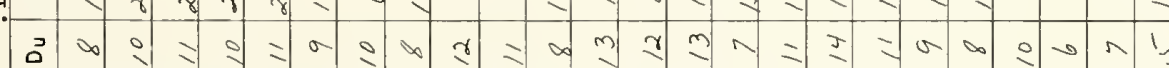

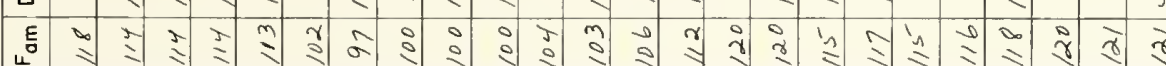

$\frac{1}{5}$

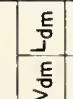

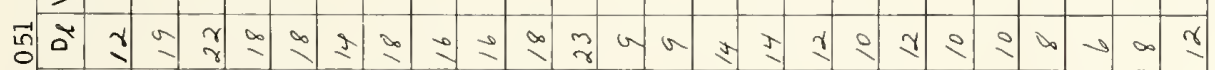

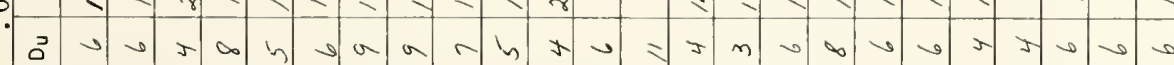

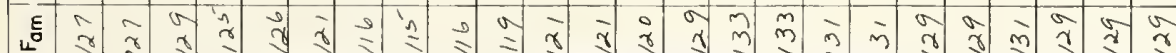

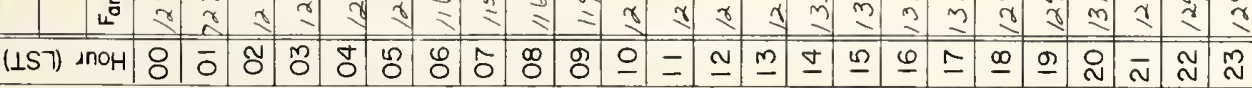

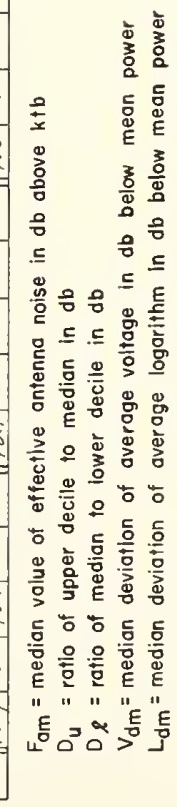




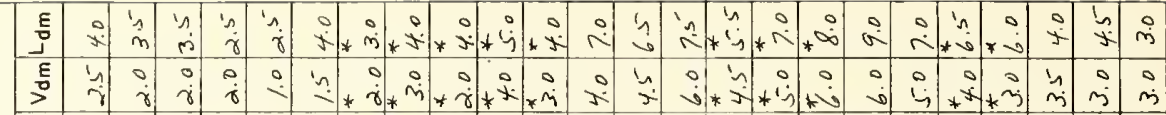

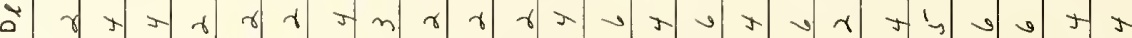

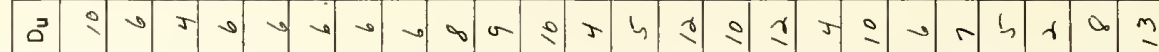

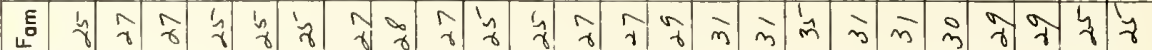
E

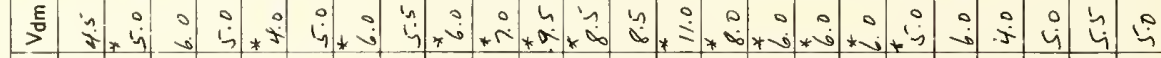
ㅇำ 年|

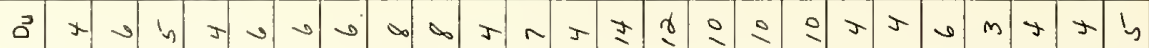

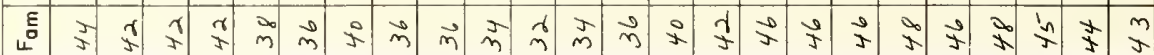

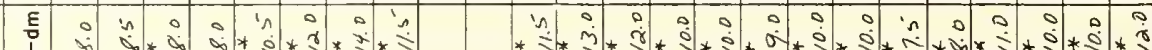
N. $n+n+\infty$

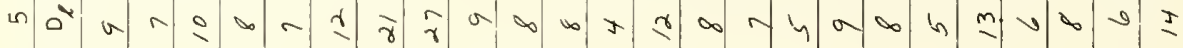

m.

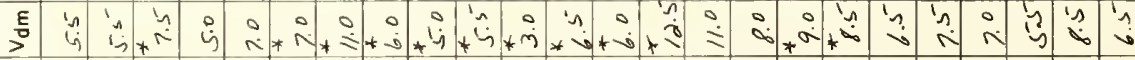

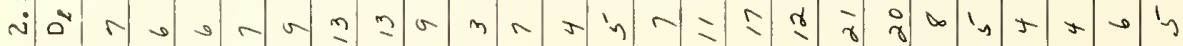
J

w
$\frac{1}{2}$
$\frac{0}{2}$
$\frac{1}{1}$

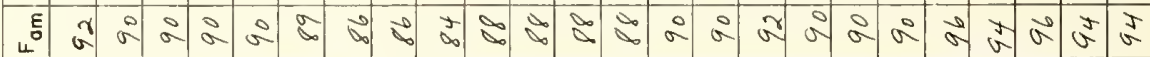

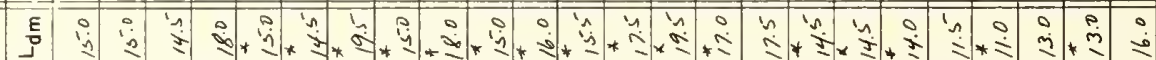

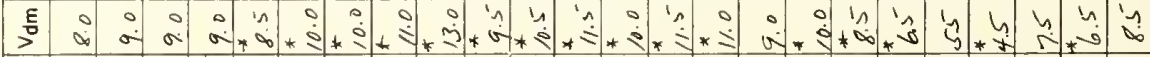

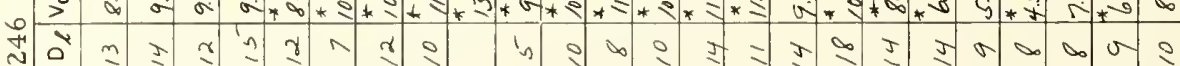

एँ

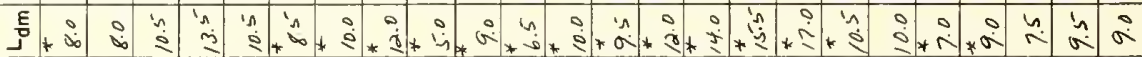

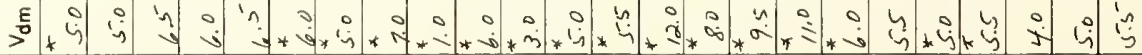
๑ั 


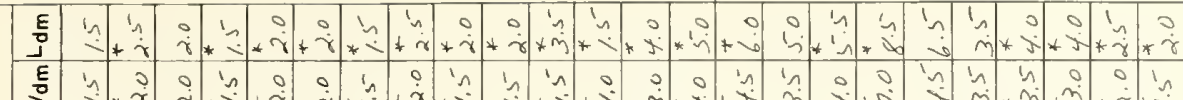

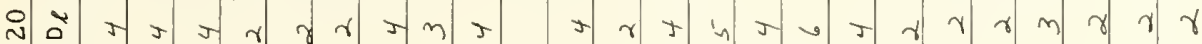

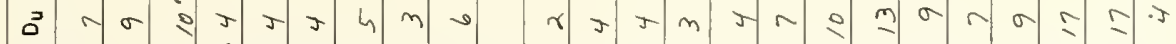

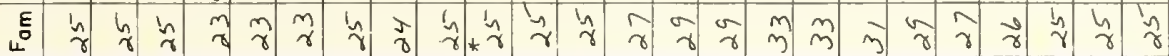

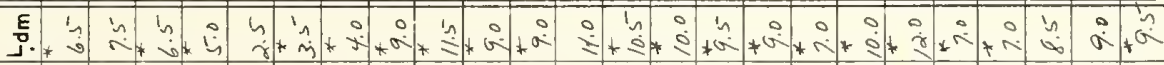

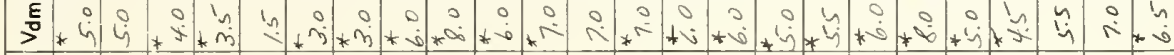

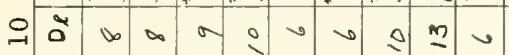

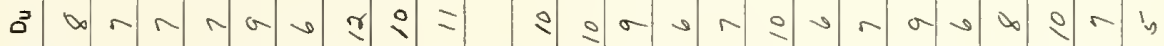

Е है मे भे

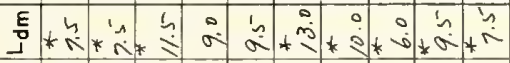

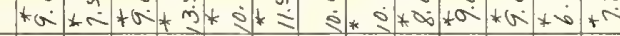

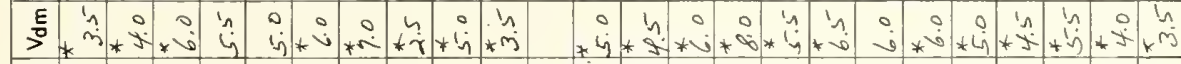

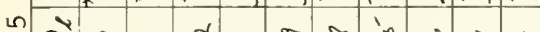

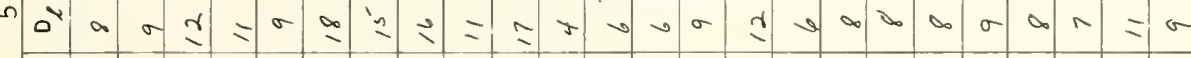

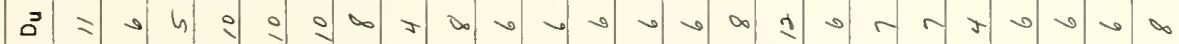

है

+艹

至

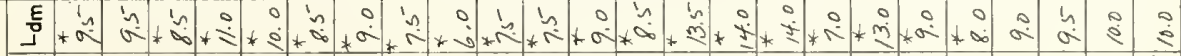
ह i

ב E 첨

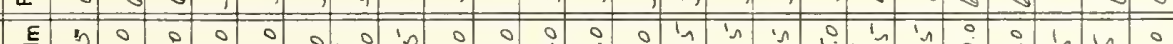

j.

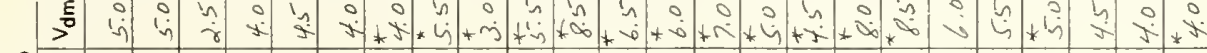
กी

(3) $\pi$

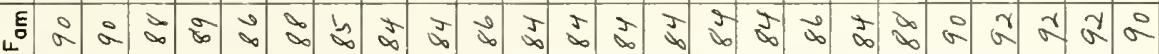

$\frac{w}{2}$

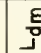

E

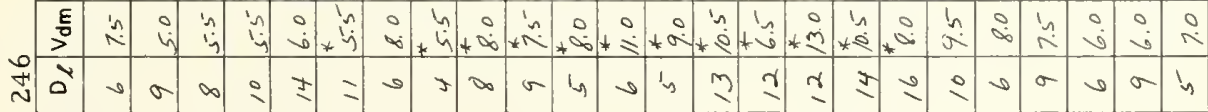

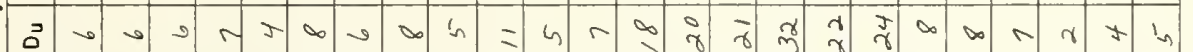
है

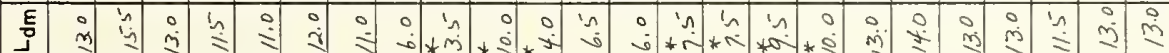

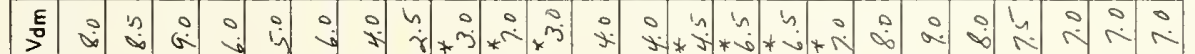

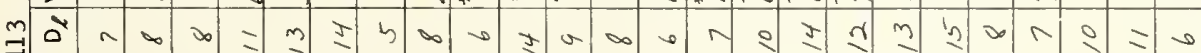
$\because$

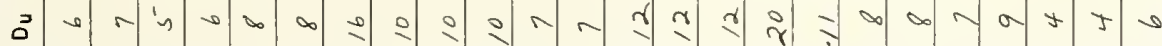
ह

责

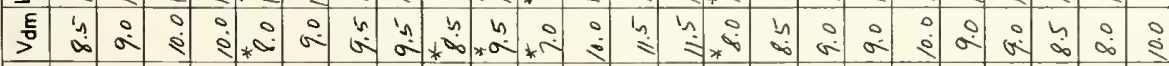

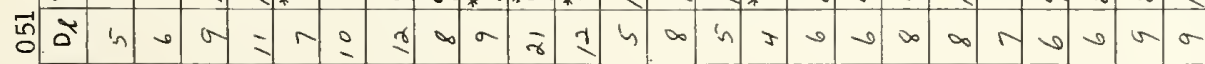

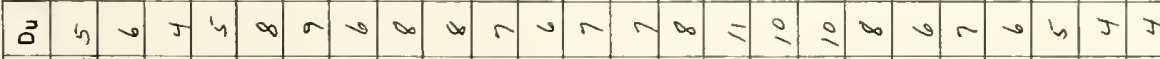

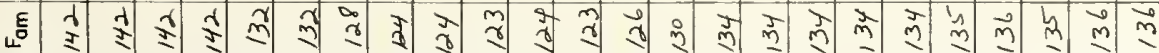

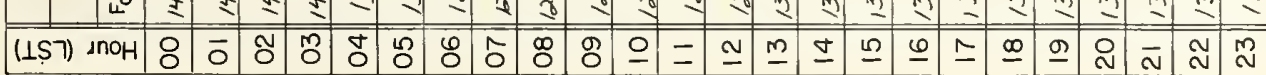




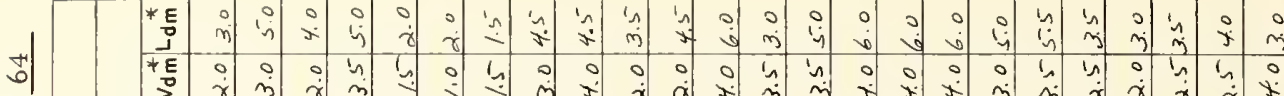

위ำ

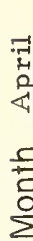

a

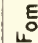

* 틈

ને $ર$ રે

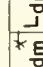
$\stackrel{-1}{\circ}$

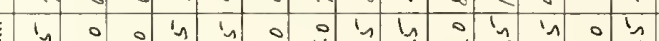

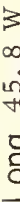

s

(2)

ल.|

क्ष

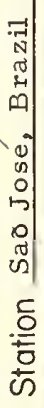

(⿸\zh14口

*E

(2)

o 4

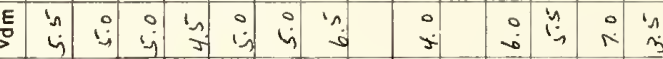

ב

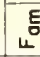

FE

*E in

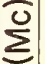

$\overrightarrow{0}$

E

청

马े in 0

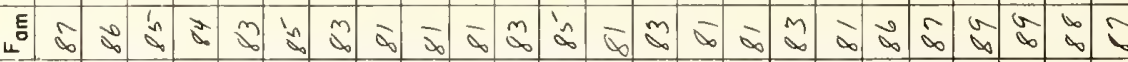

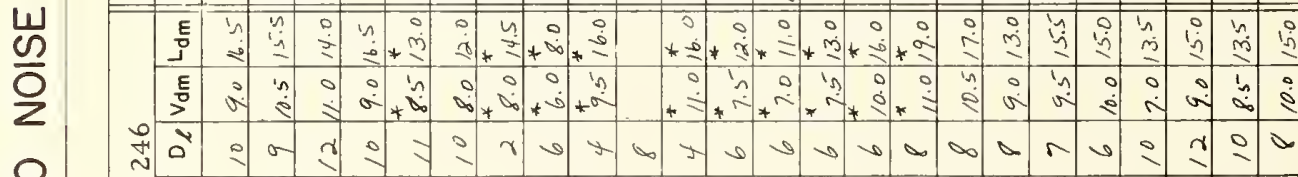

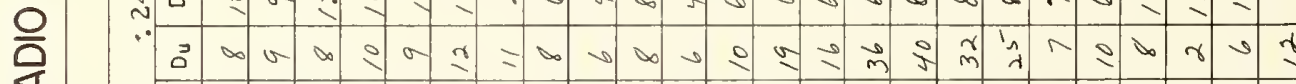

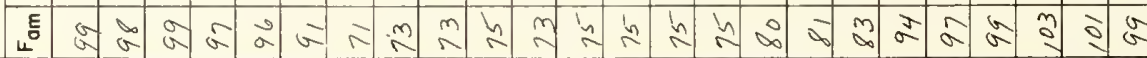

$\overleftarrow{1}$

แั

先

E

㞷

$\stackrel{m}{=}$

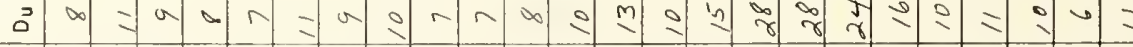

น

$s$

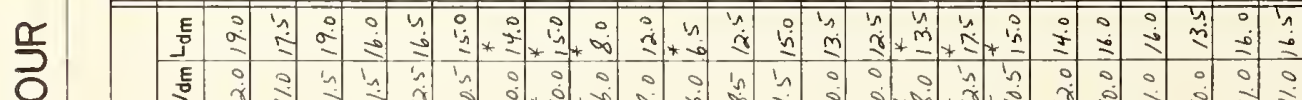

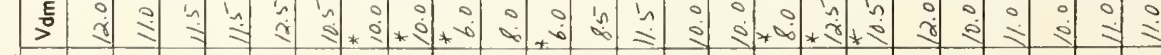

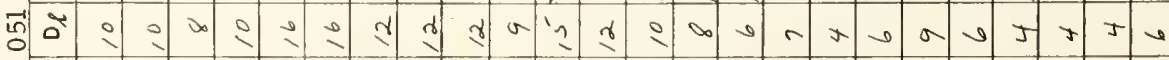

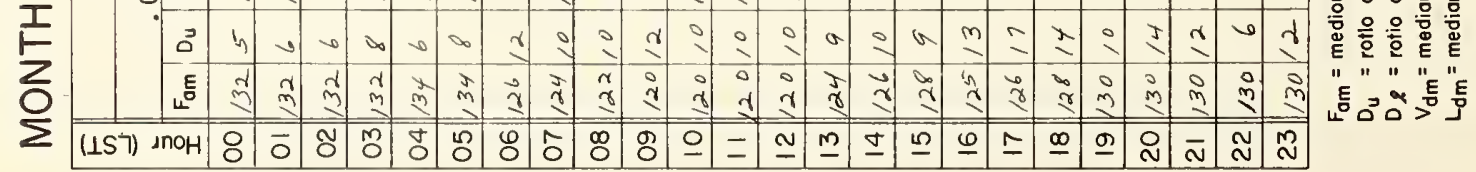




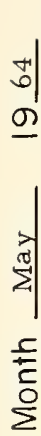

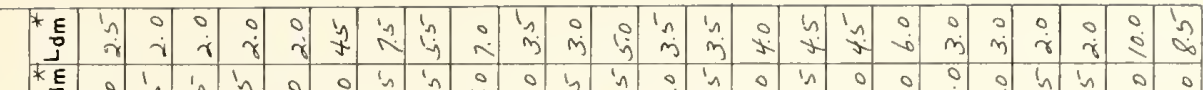

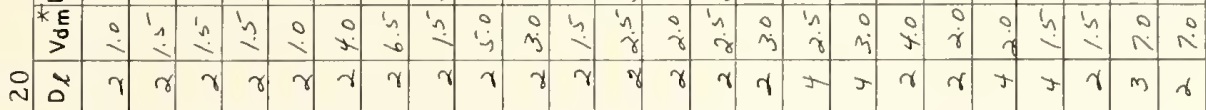

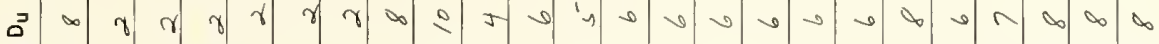

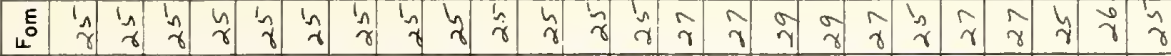

*

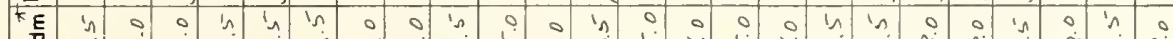

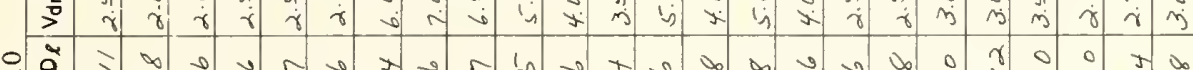

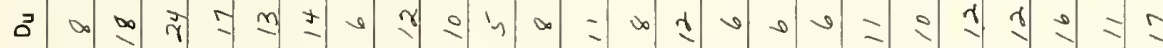
3

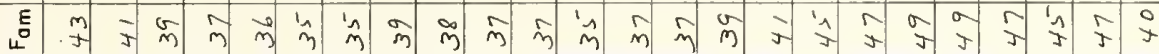

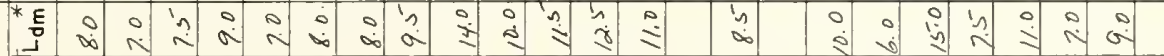

ถ

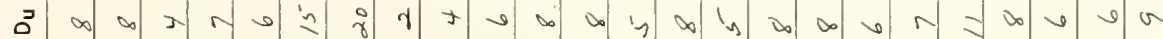

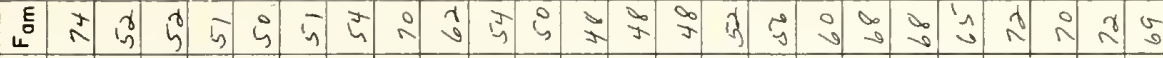

宁

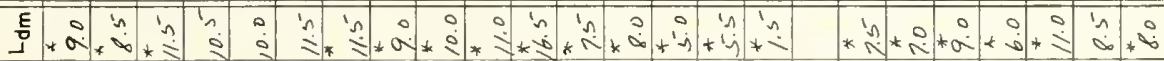

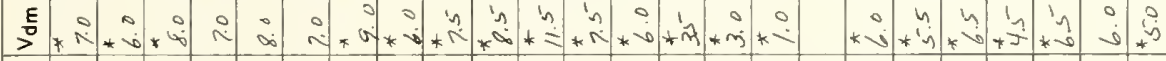

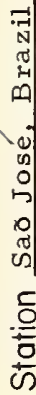

$\sum$ in

$\sum$

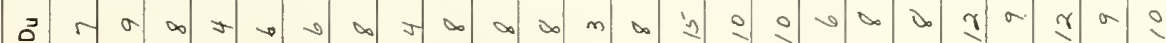

镸

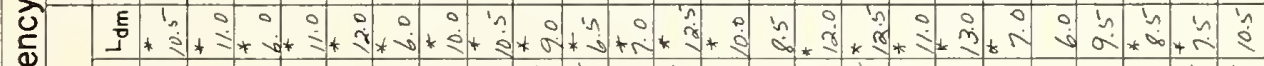

들

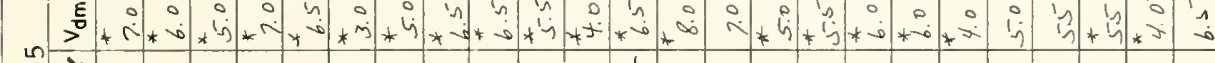

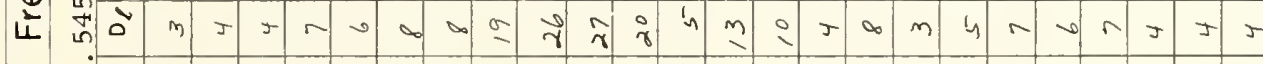

o 4 a

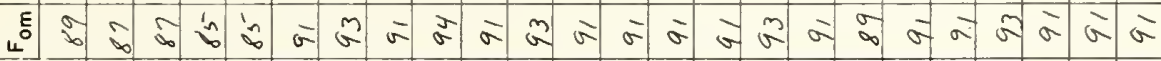

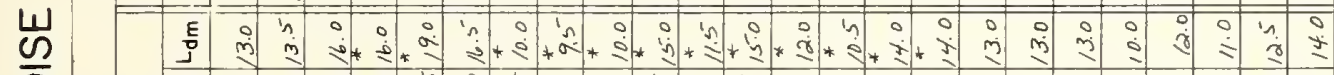

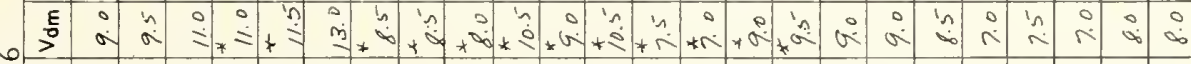

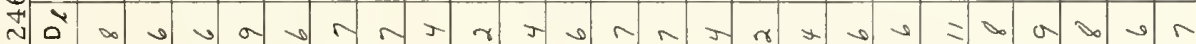

D.

จา ป⿻

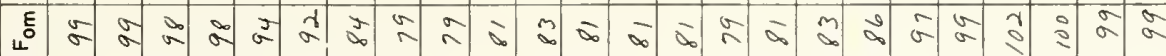

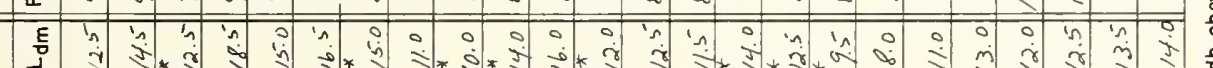

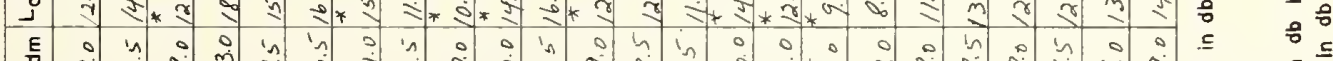

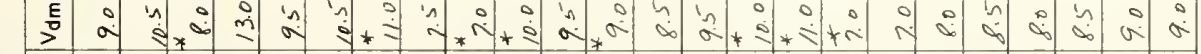

.

ว

E

E 1 - 0 0 0 (1)

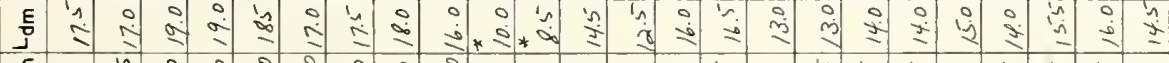

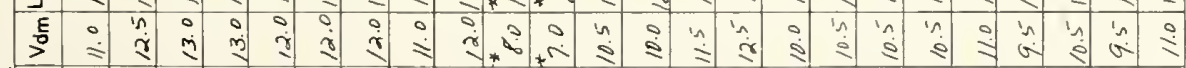

ปี 


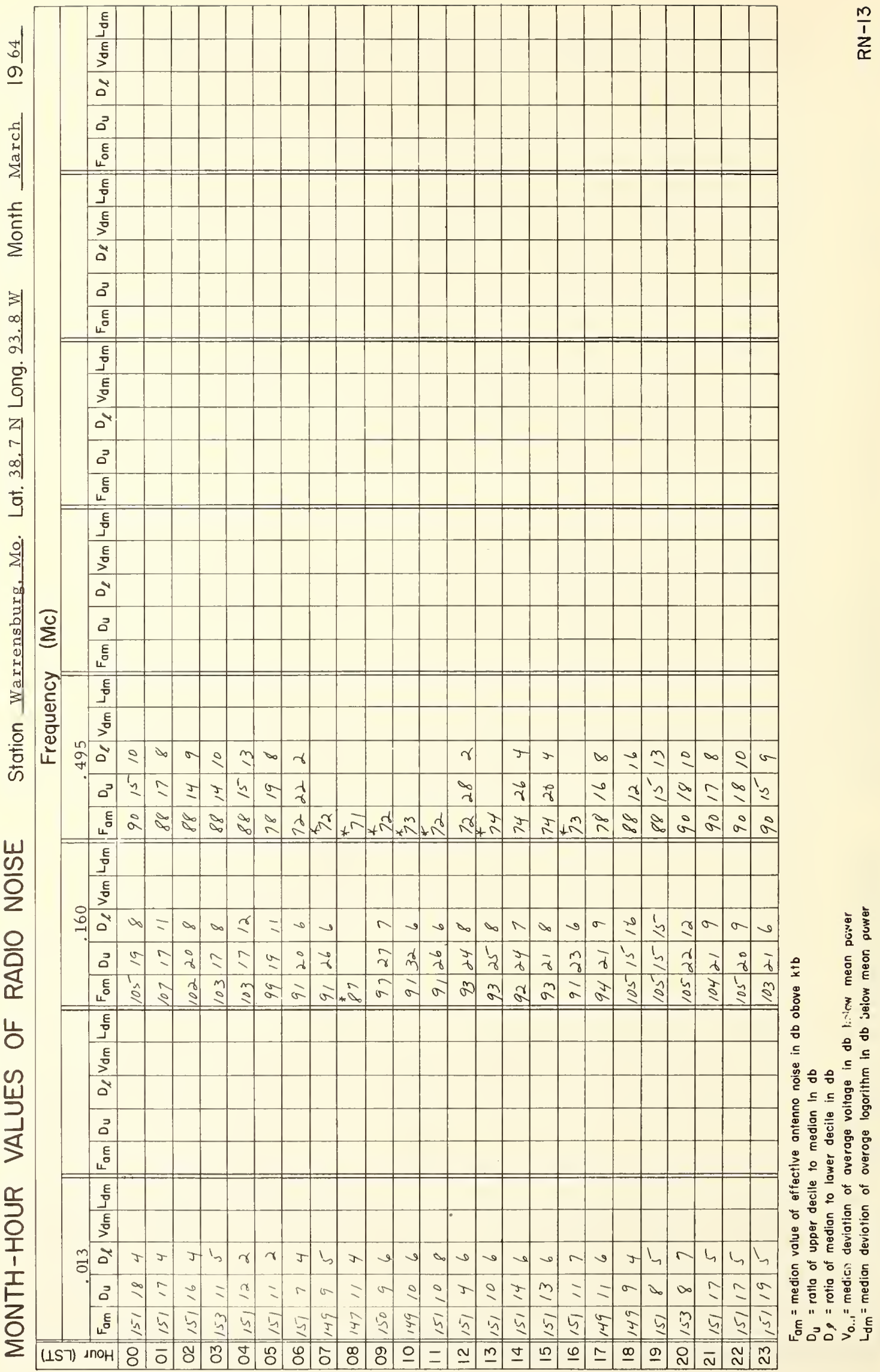




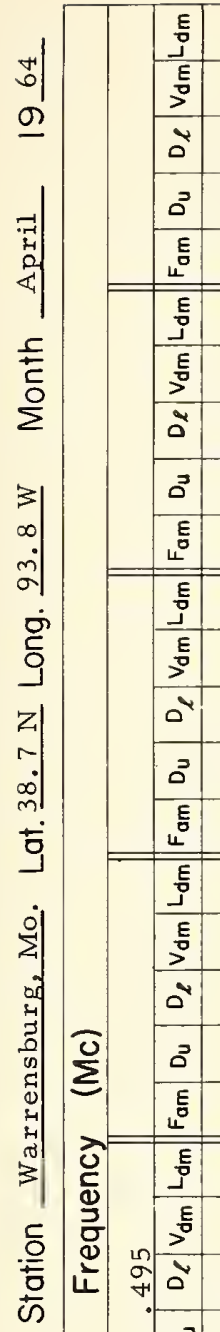

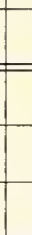

$0^{3}$

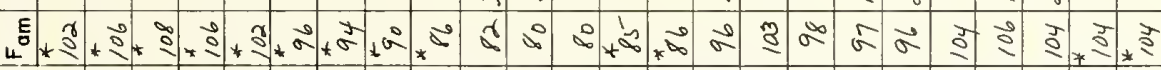

$\frac{\mathrm{w}}{\mathrm{O}}$

E

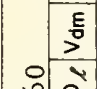

$\stackrel{1}{+}$

$\infty+\infty$

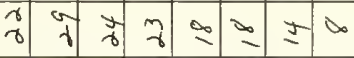

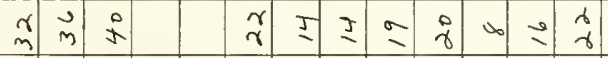




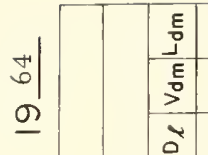

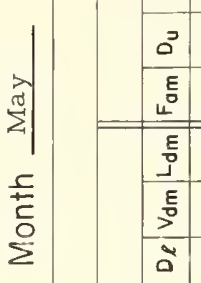

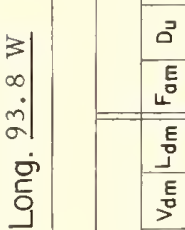

z) o

$\infty$

+ᄒ

$\dot{s}$

an

는

焉

$\sum$

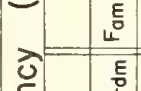

동 통

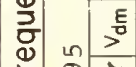

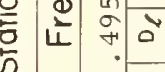

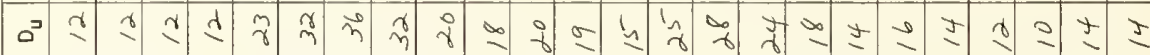

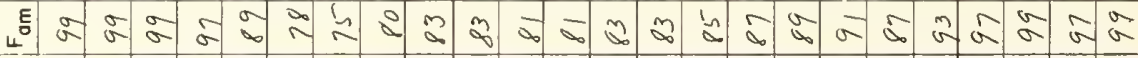

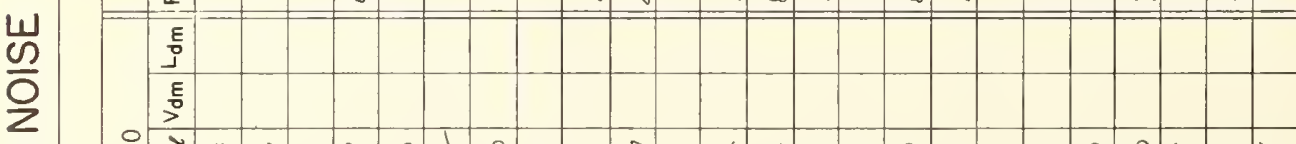

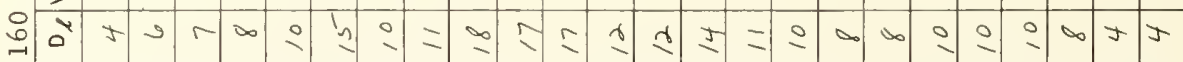

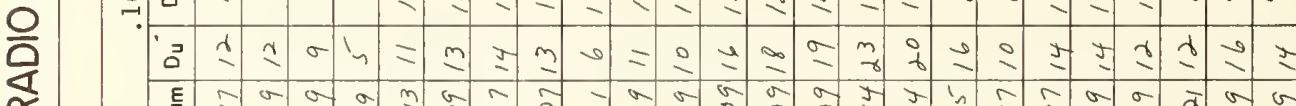

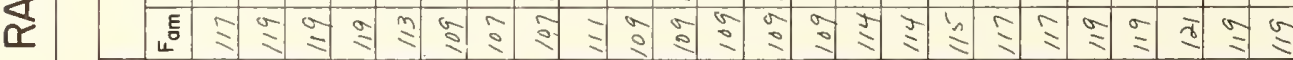

岁

E

E

岁

เี

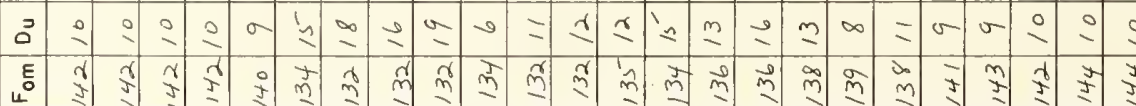

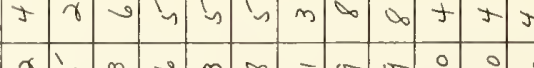

E

$\frac{1}{3}$
$\frac{0}{1}$
$\frac{1}{E}$
$\frac{2}{2}$

E

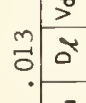

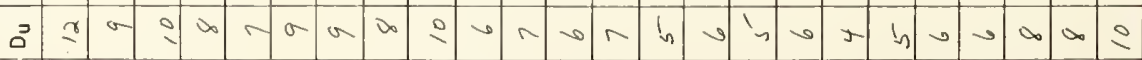

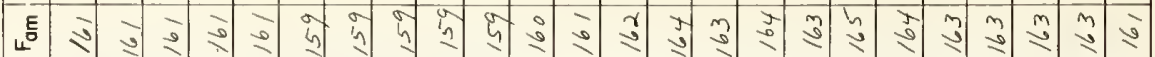

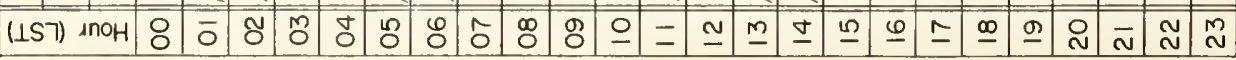

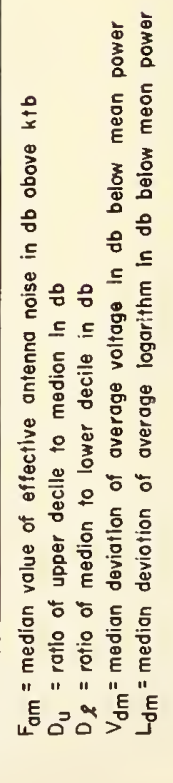




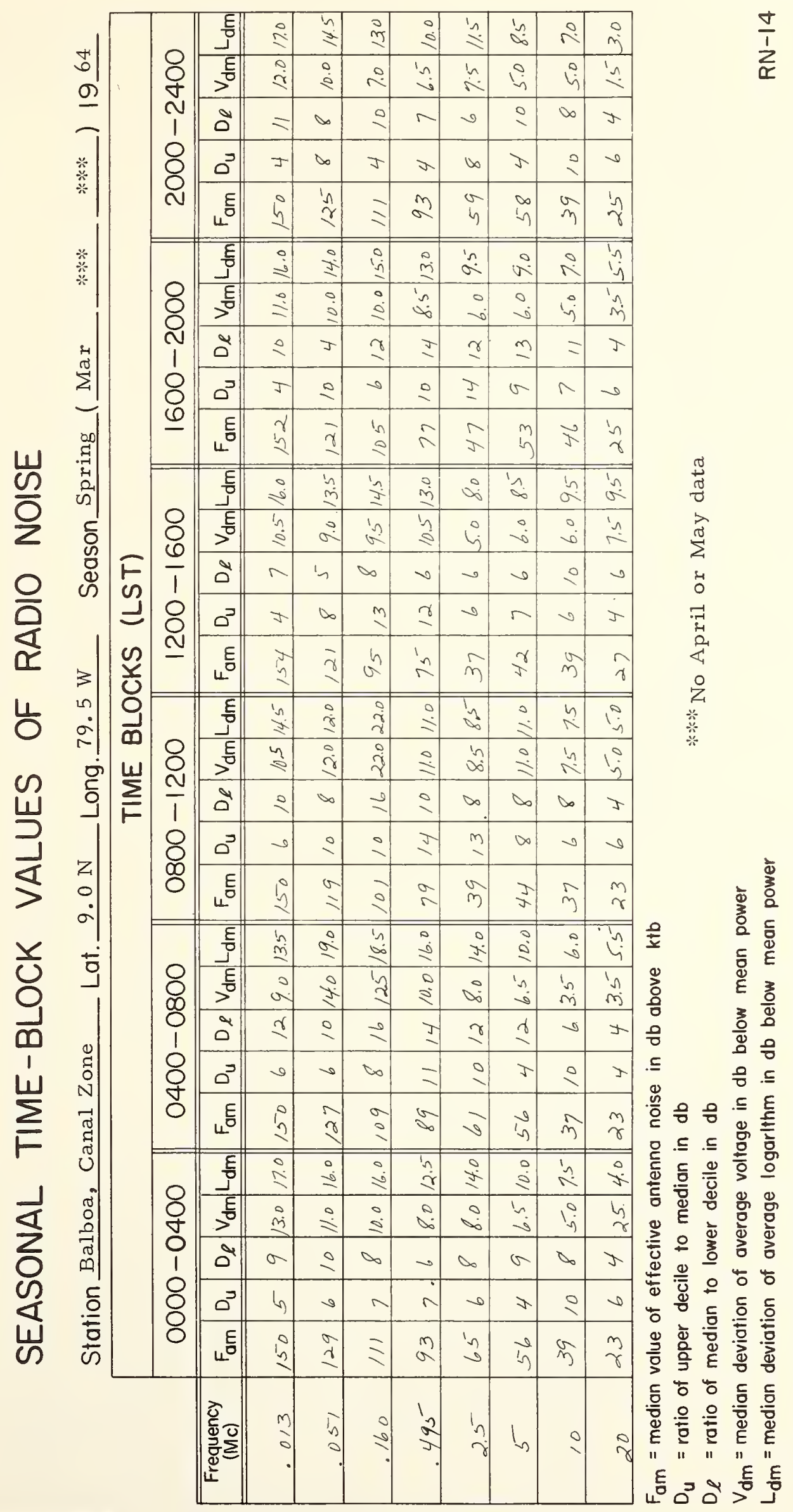




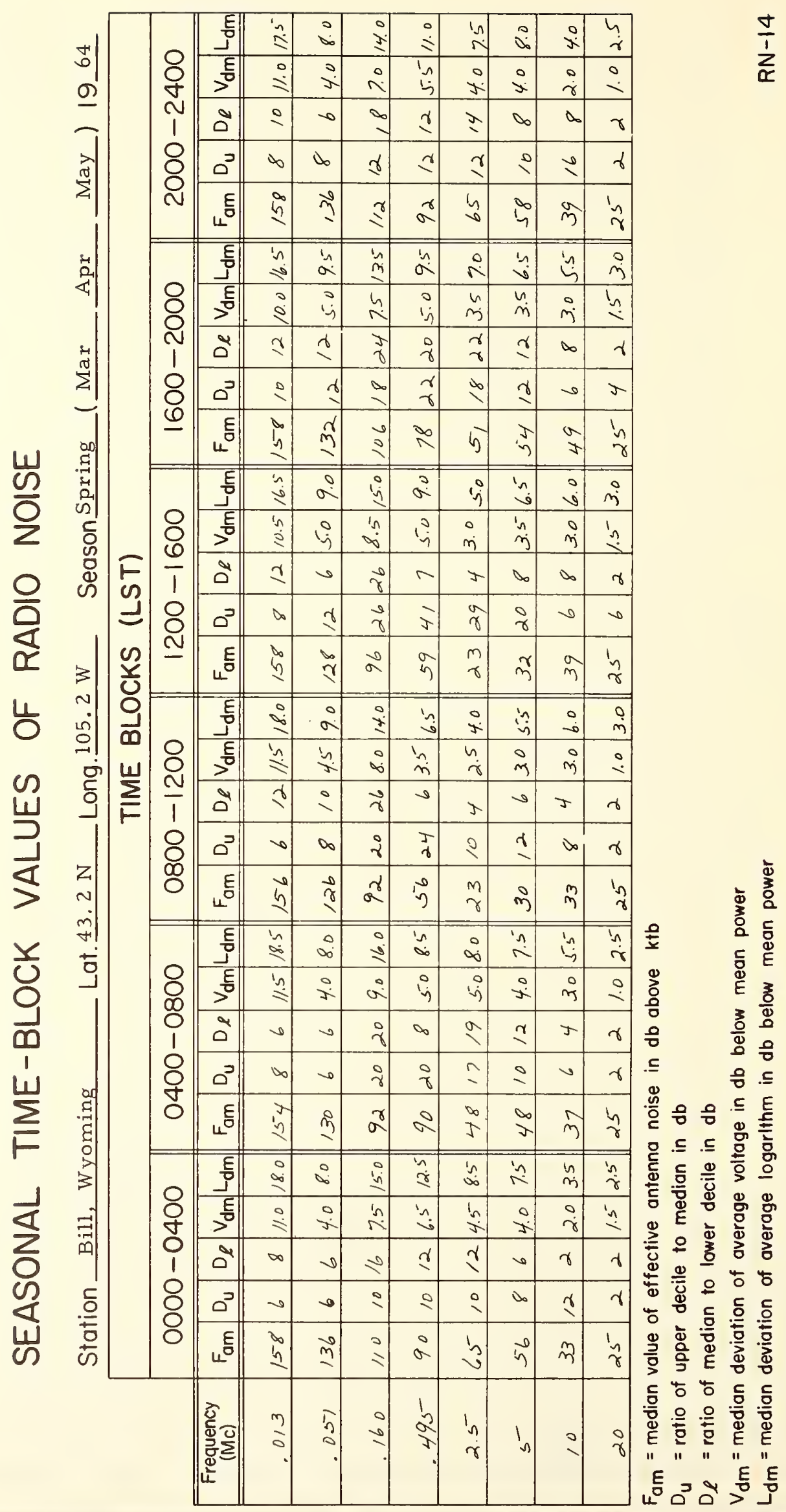




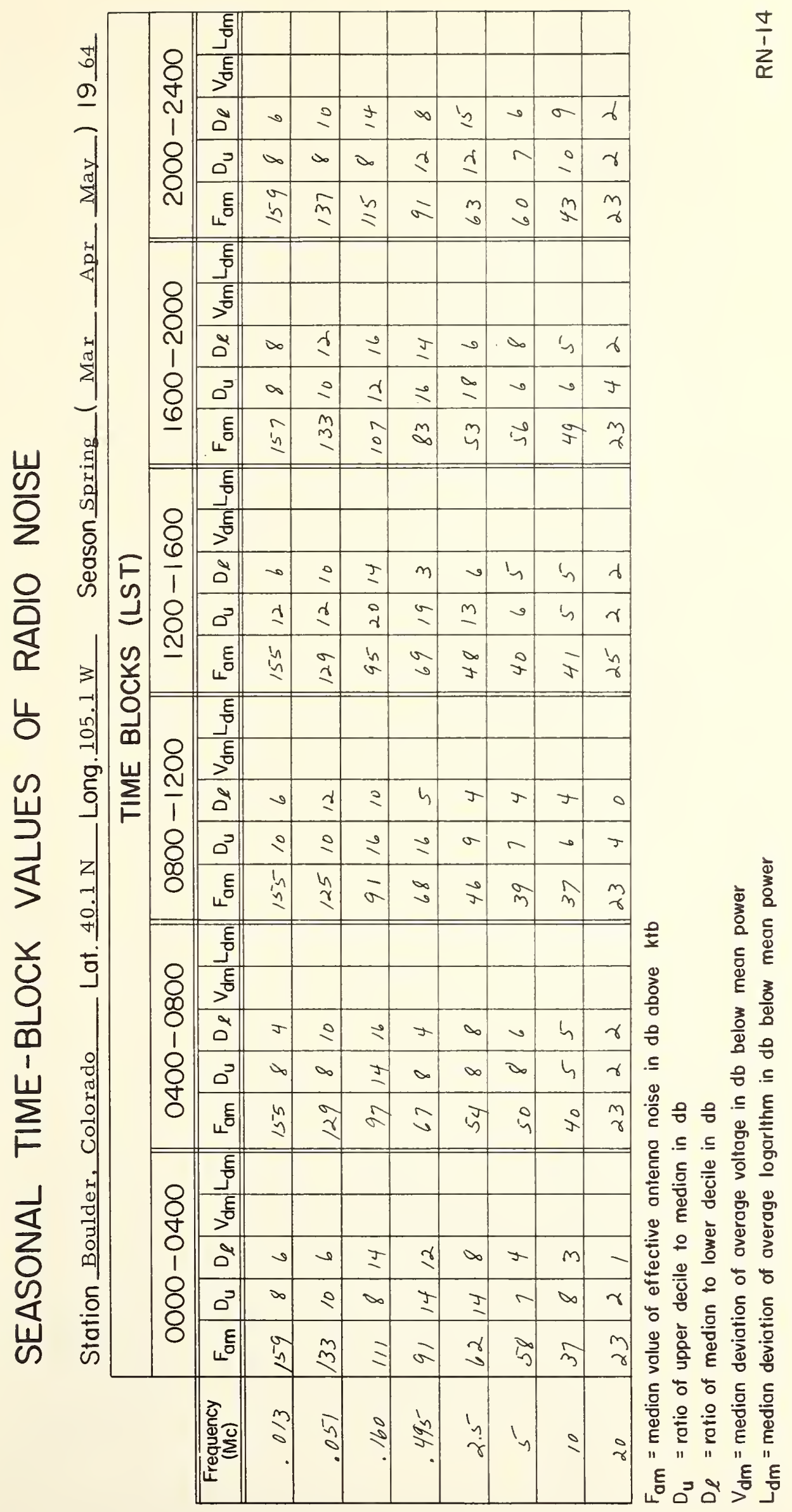




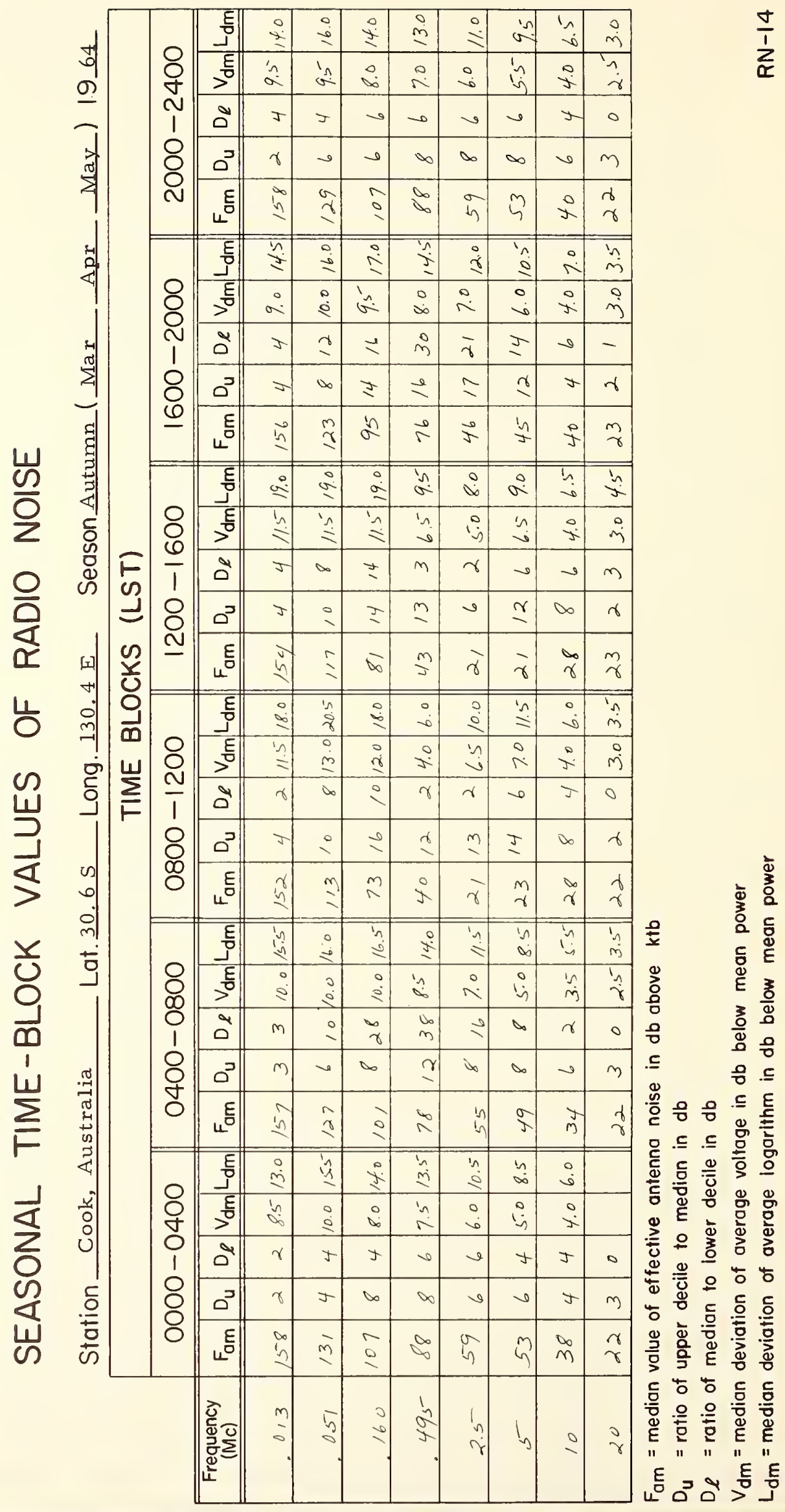




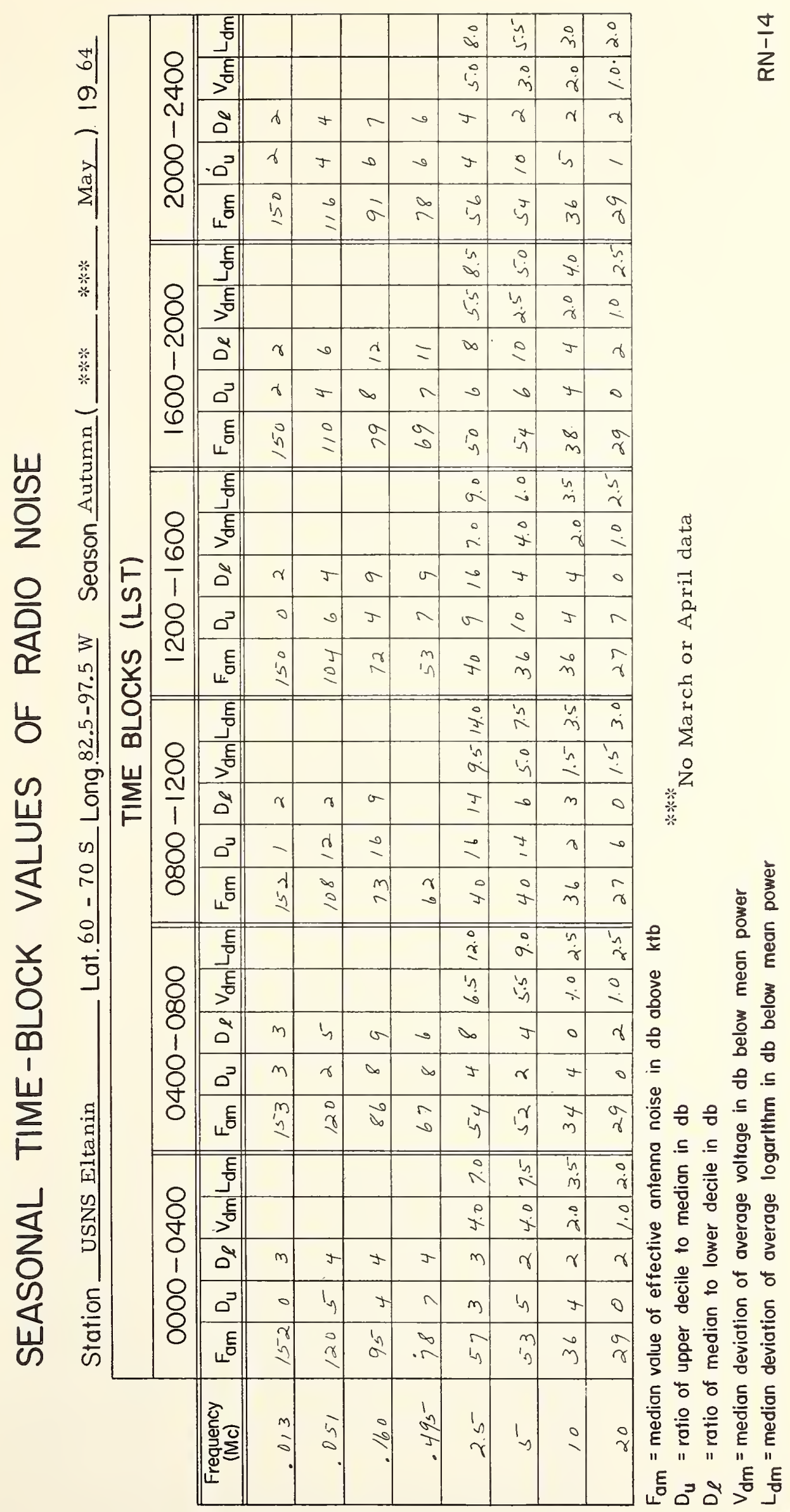




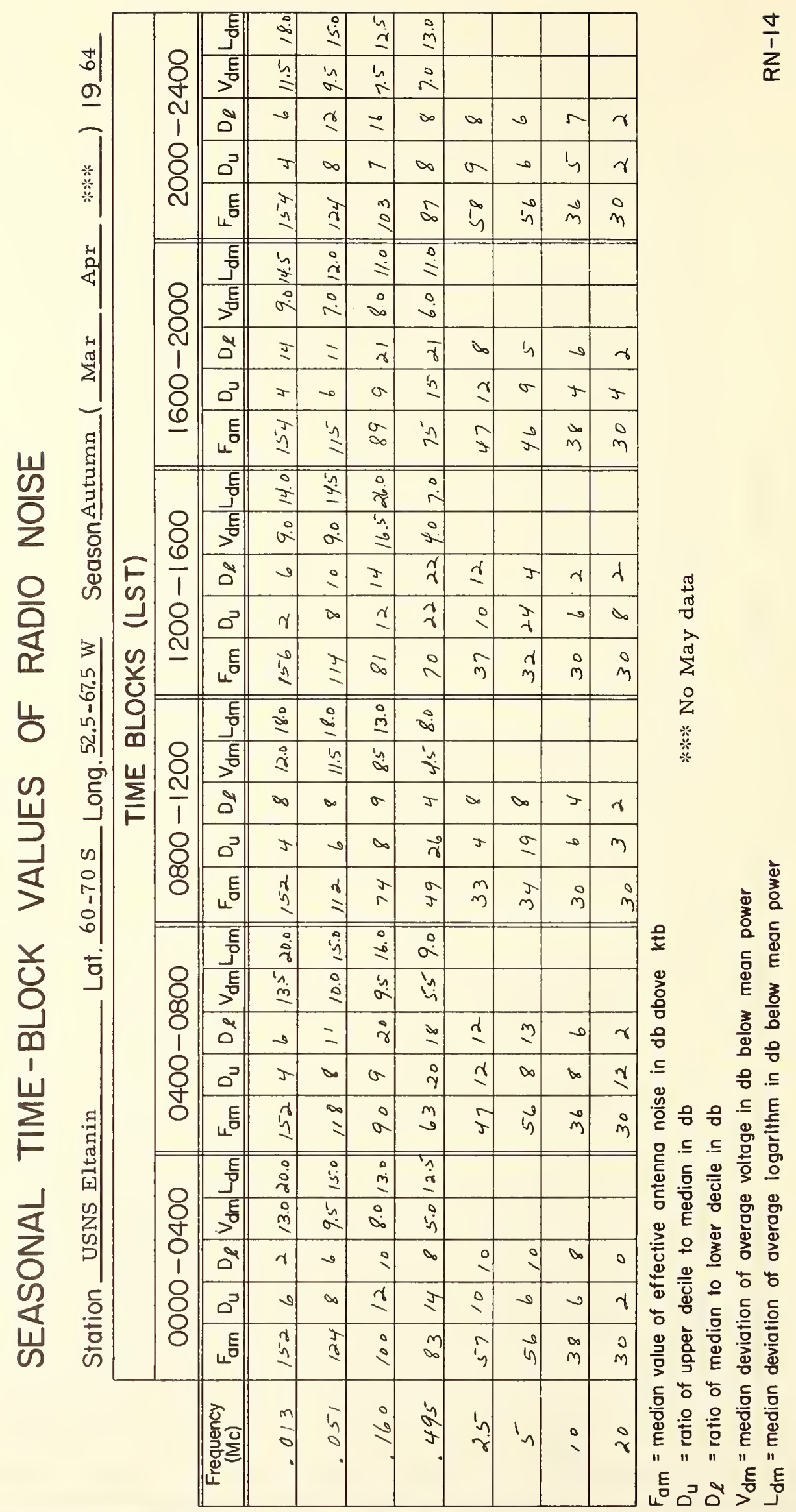




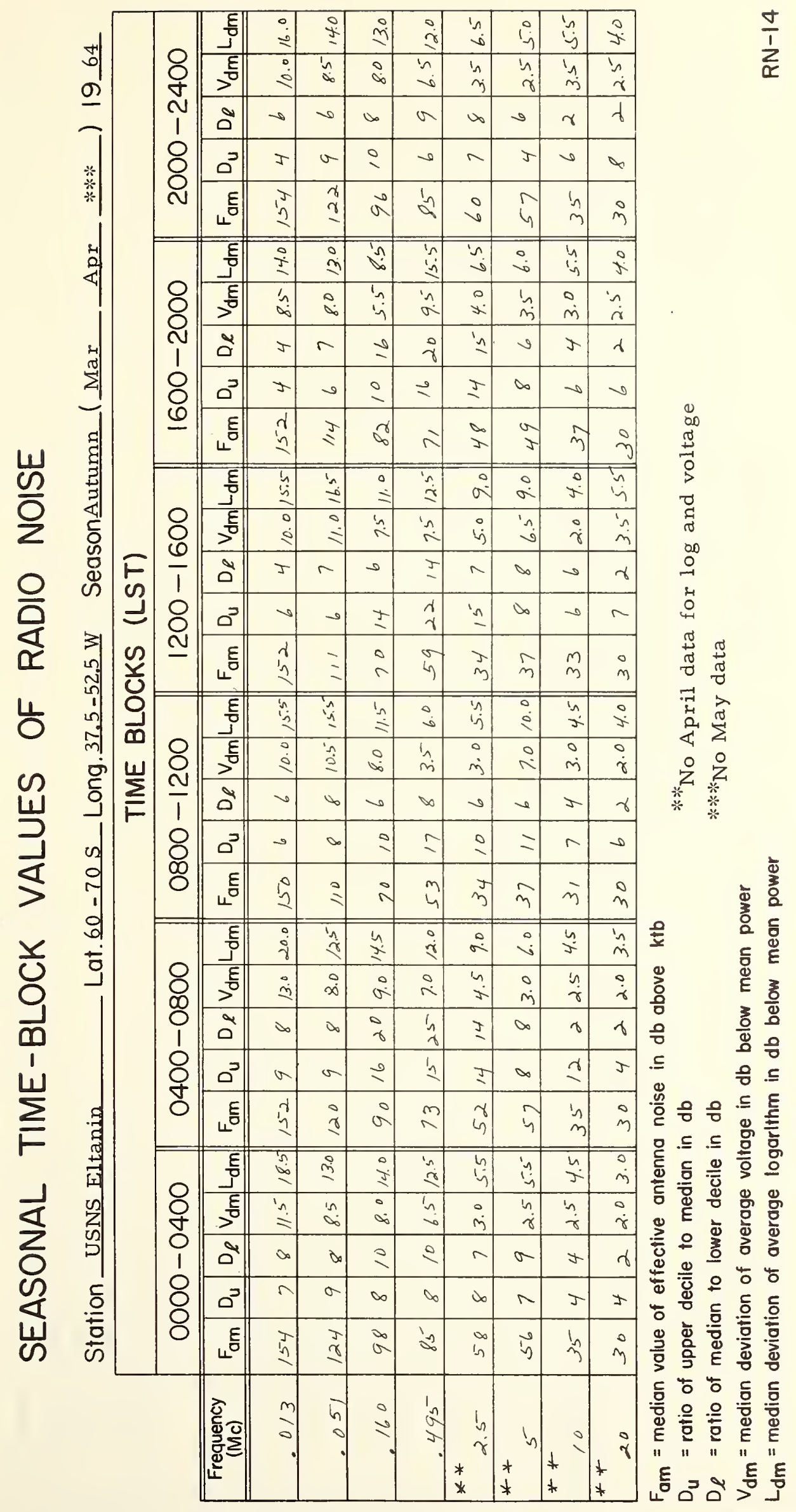




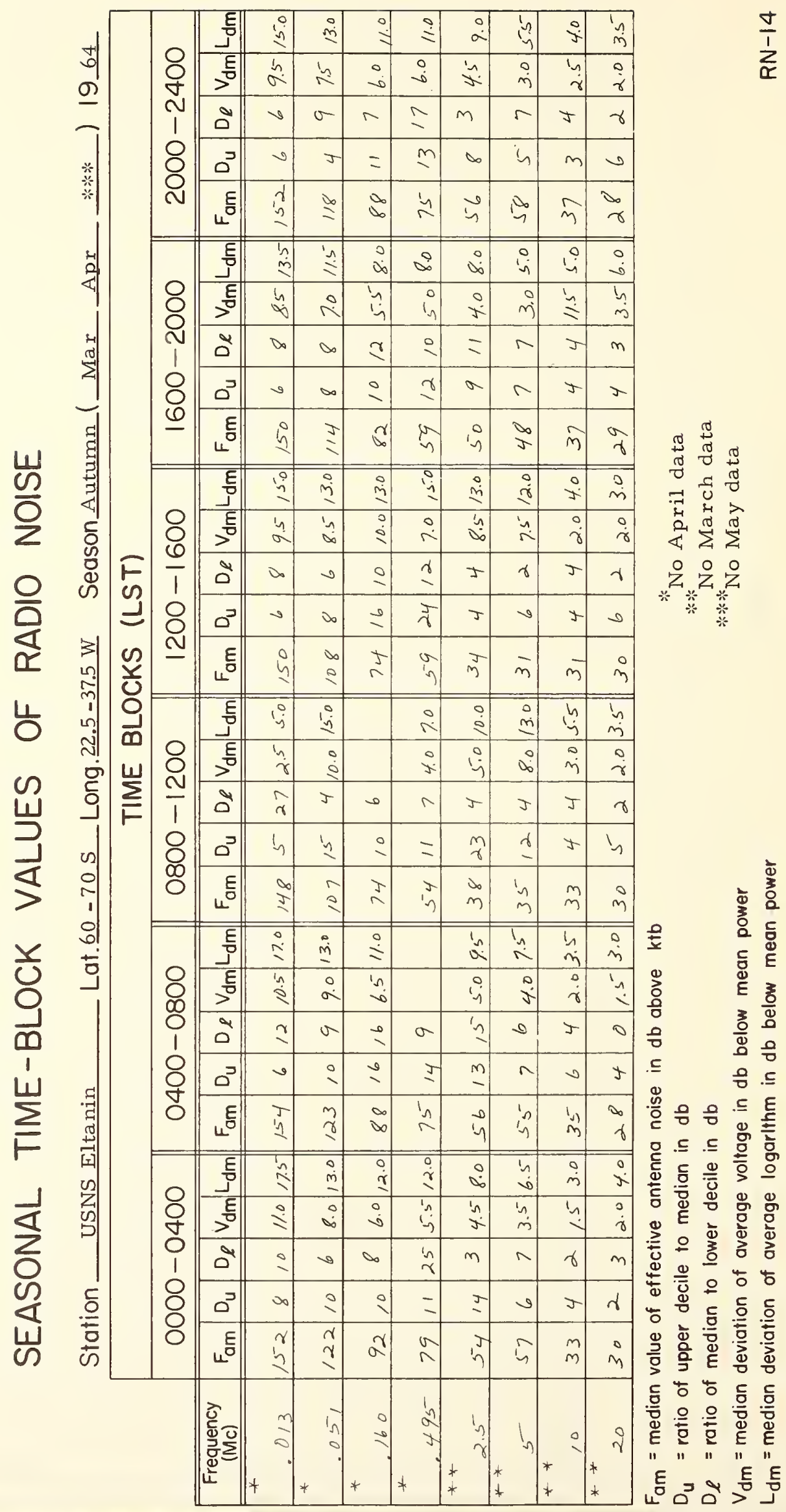




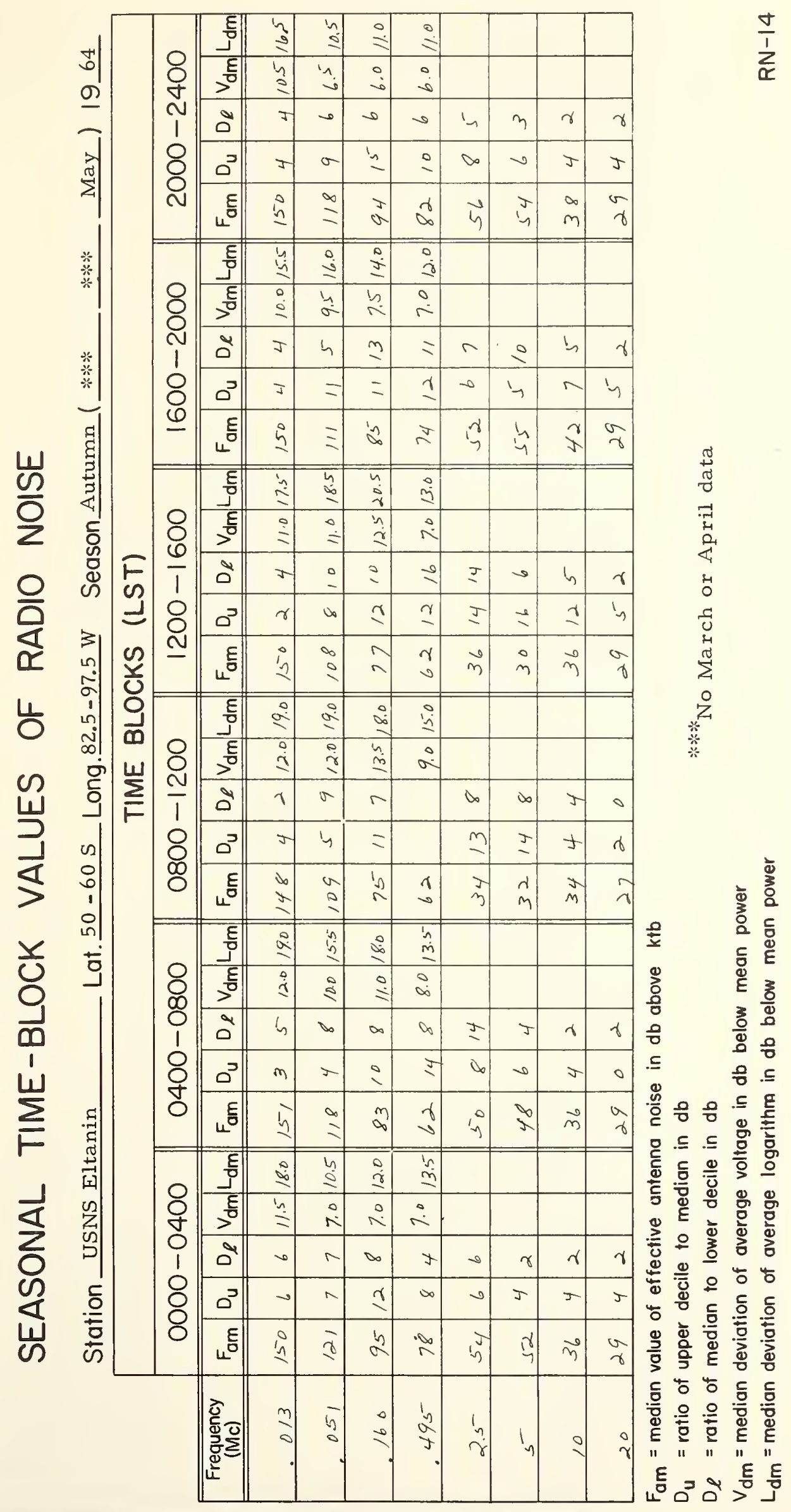




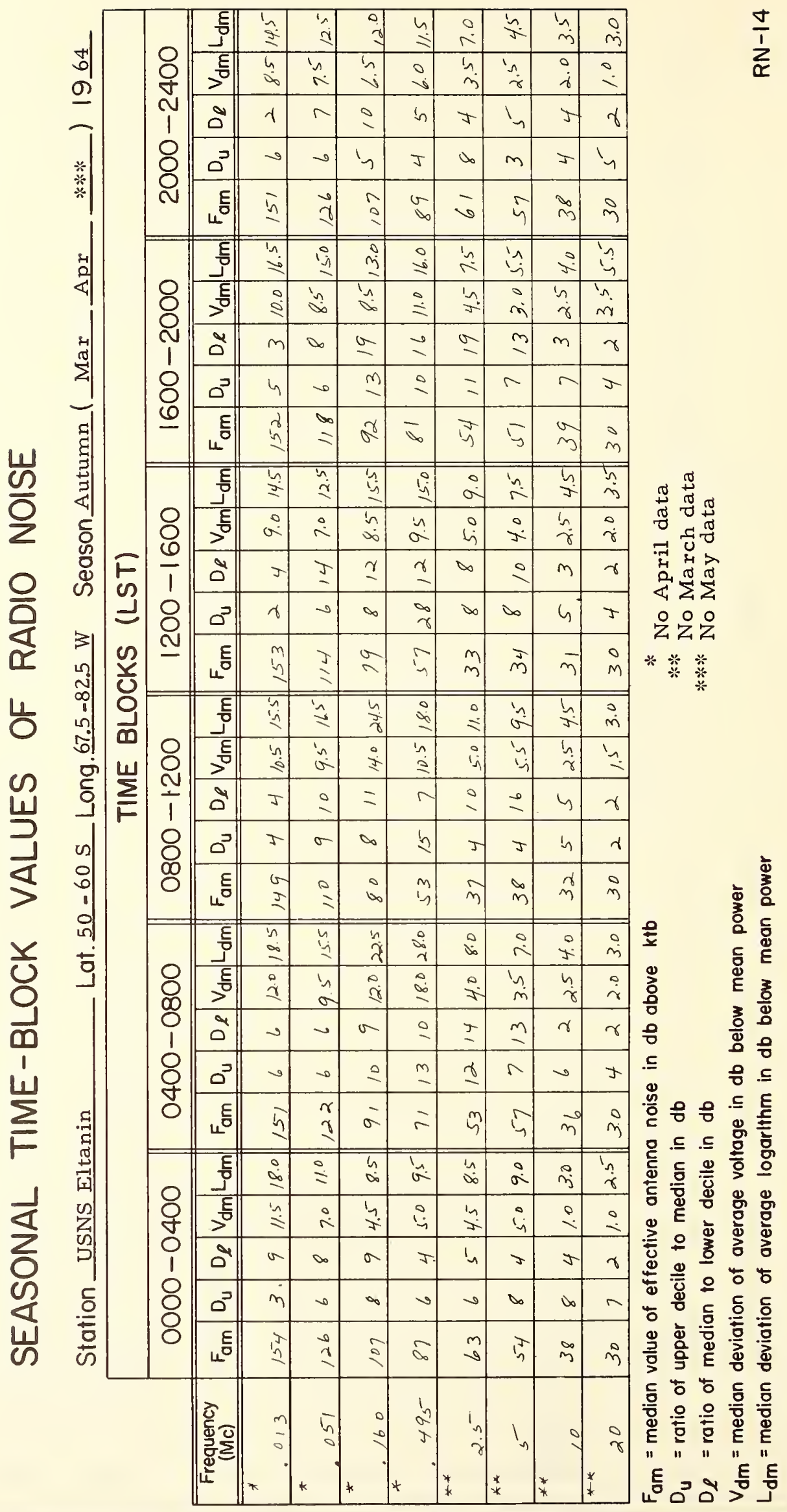




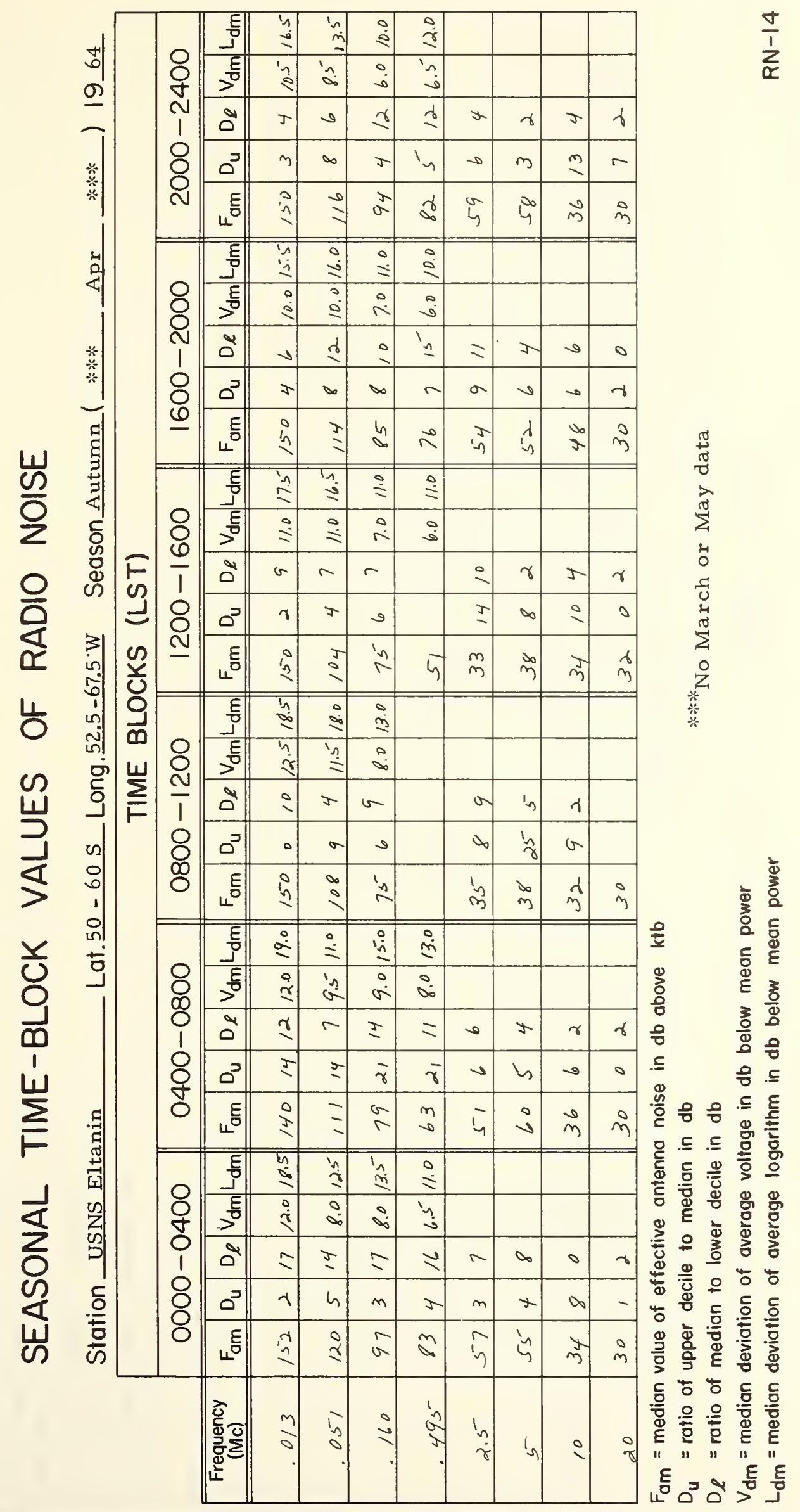




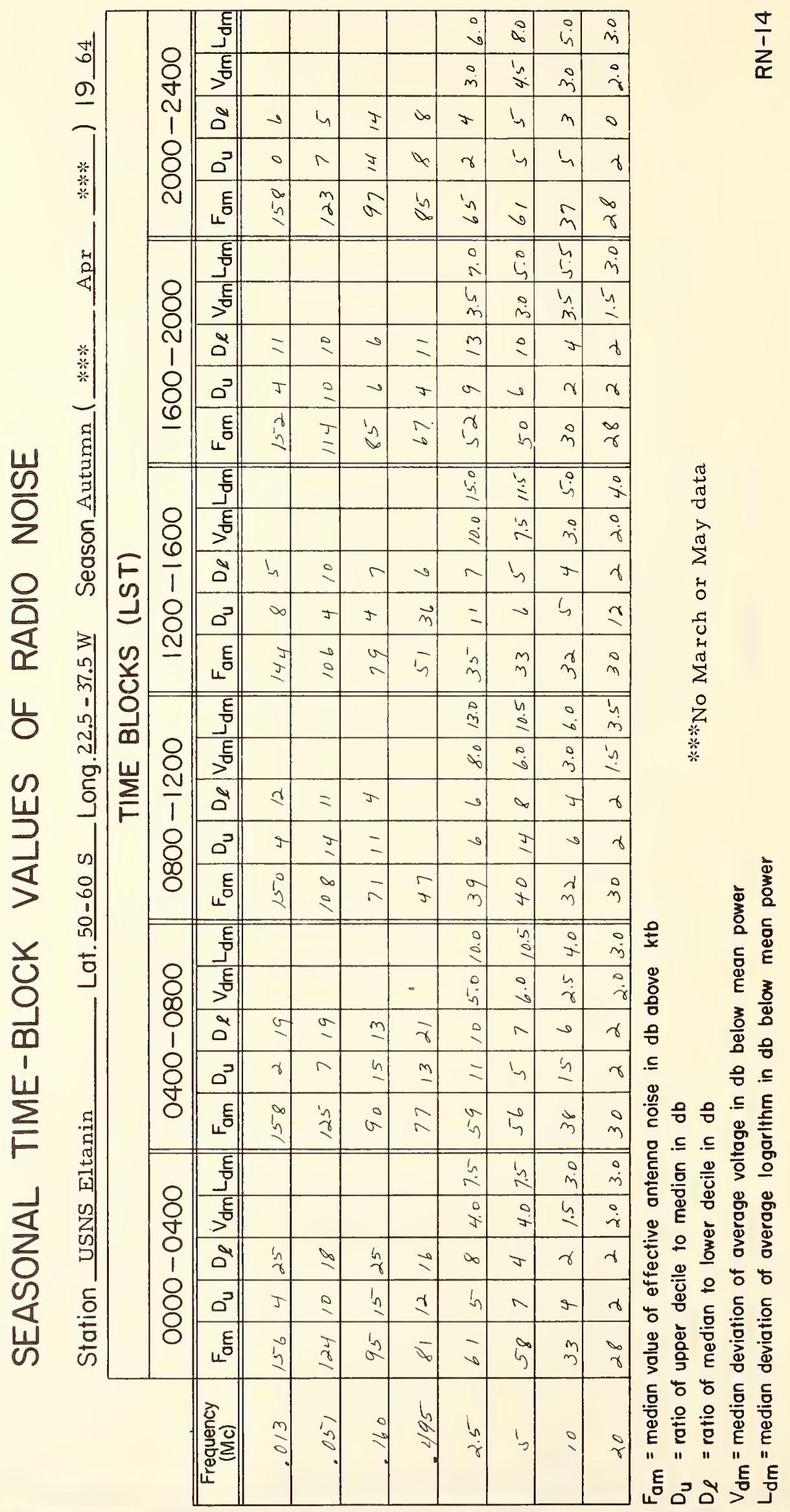




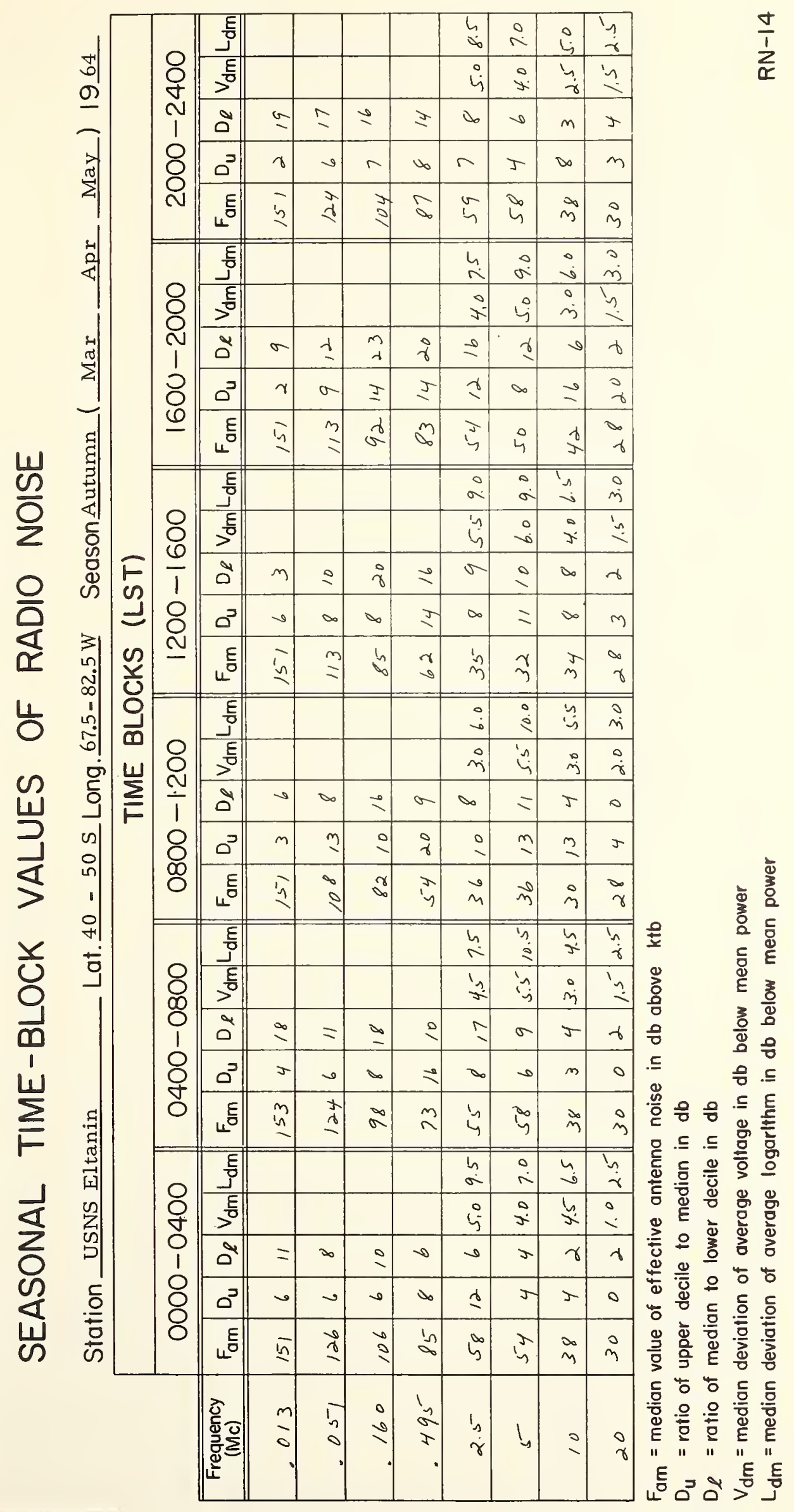




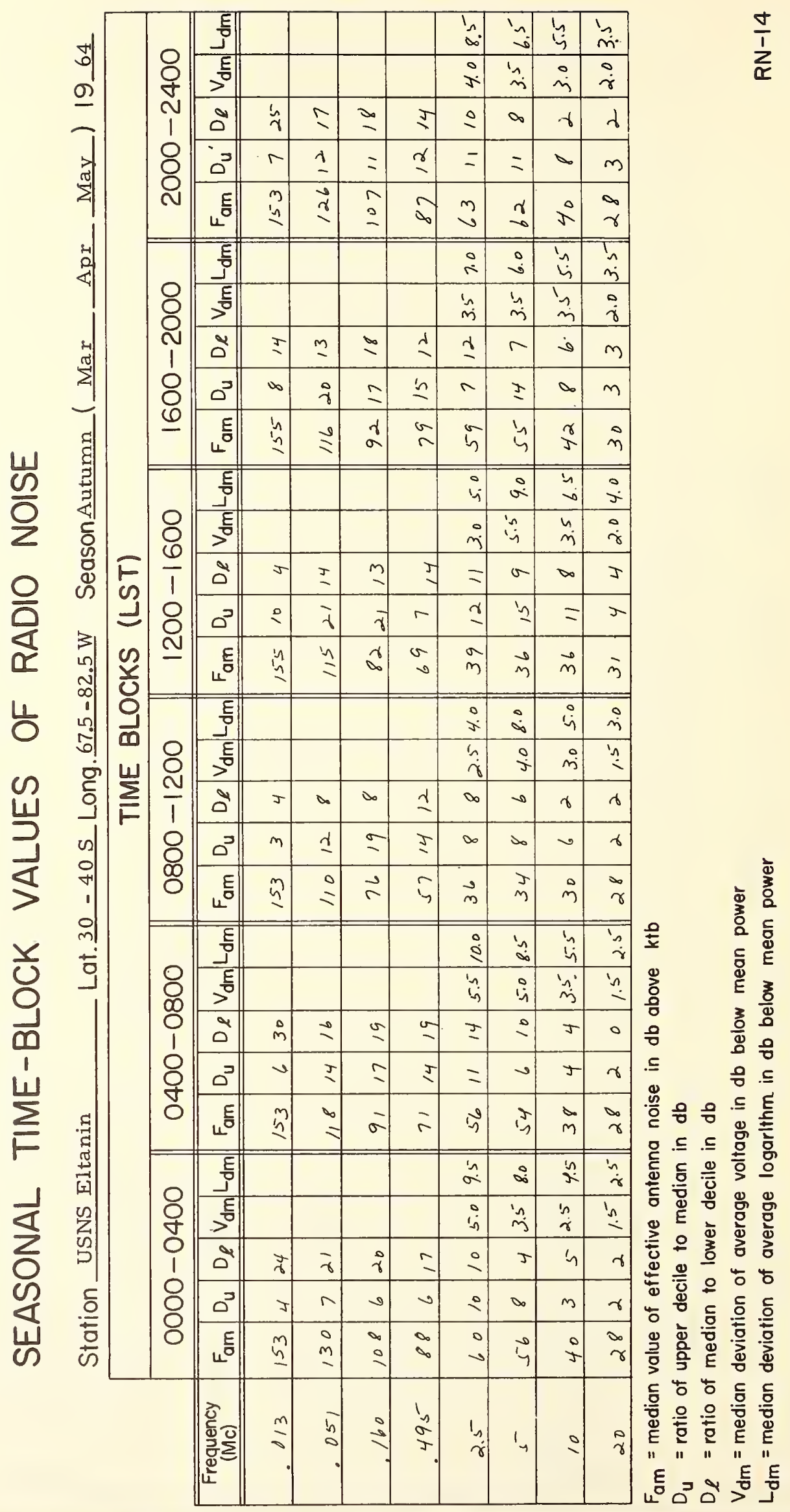




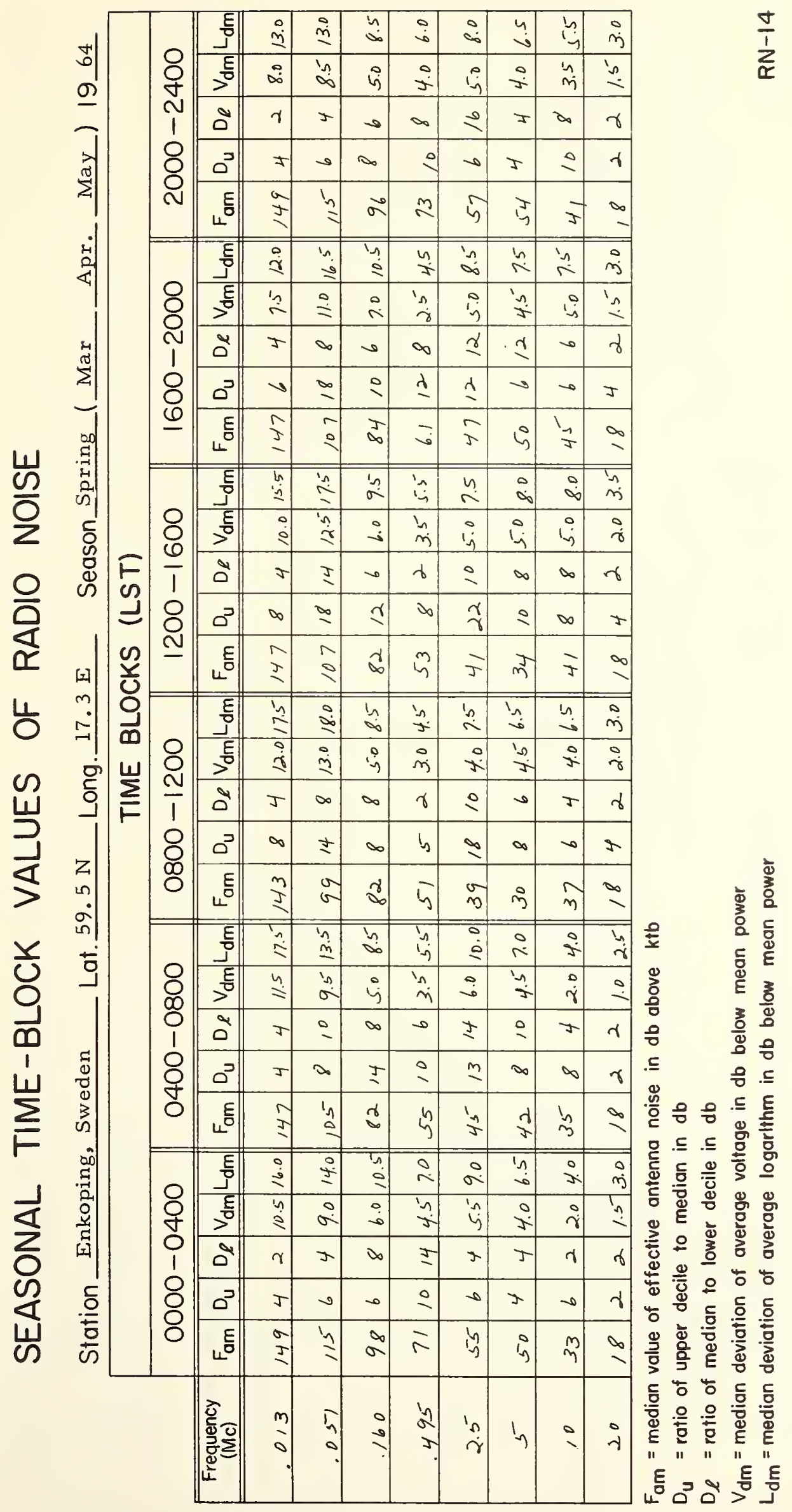




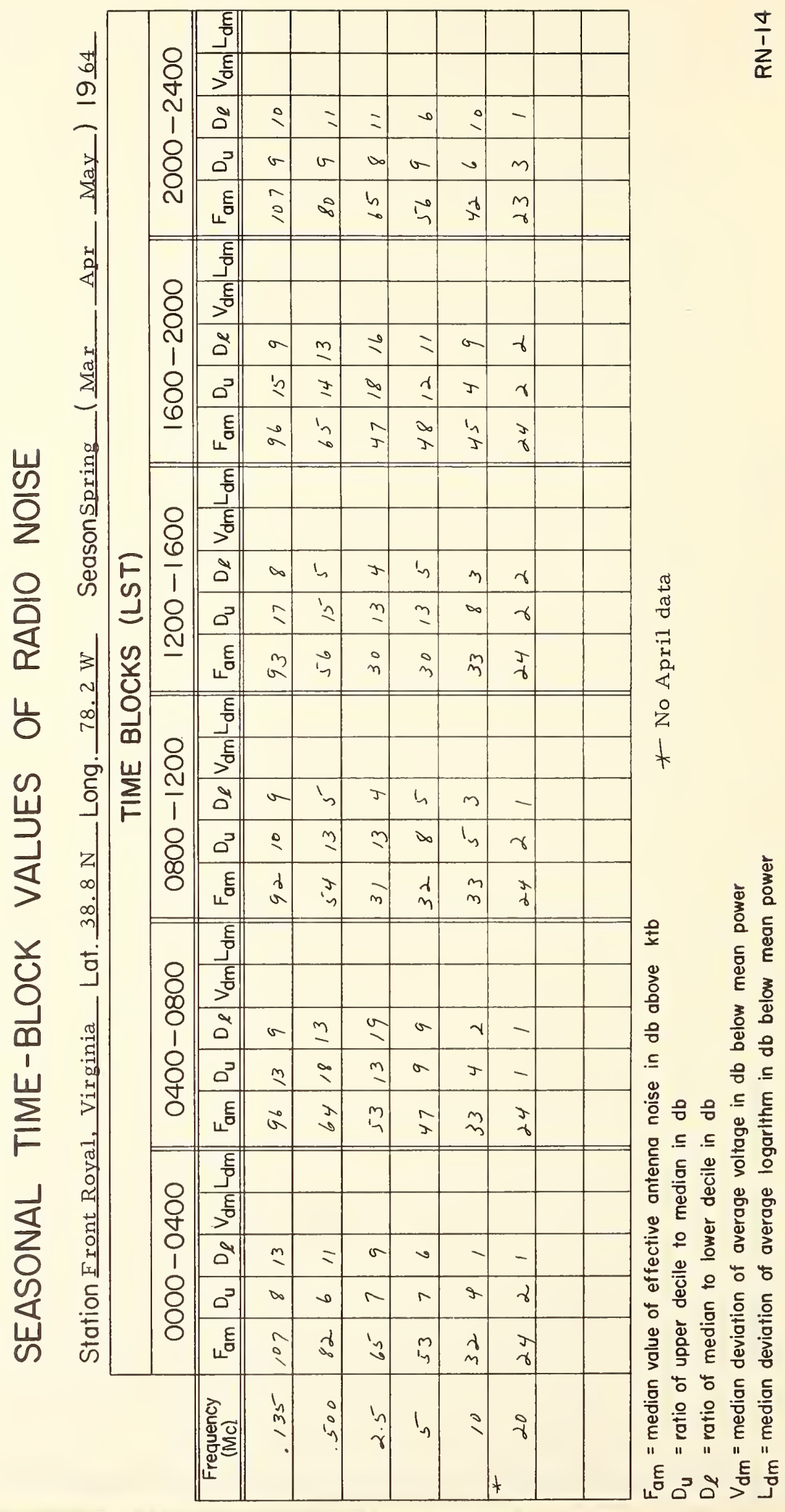




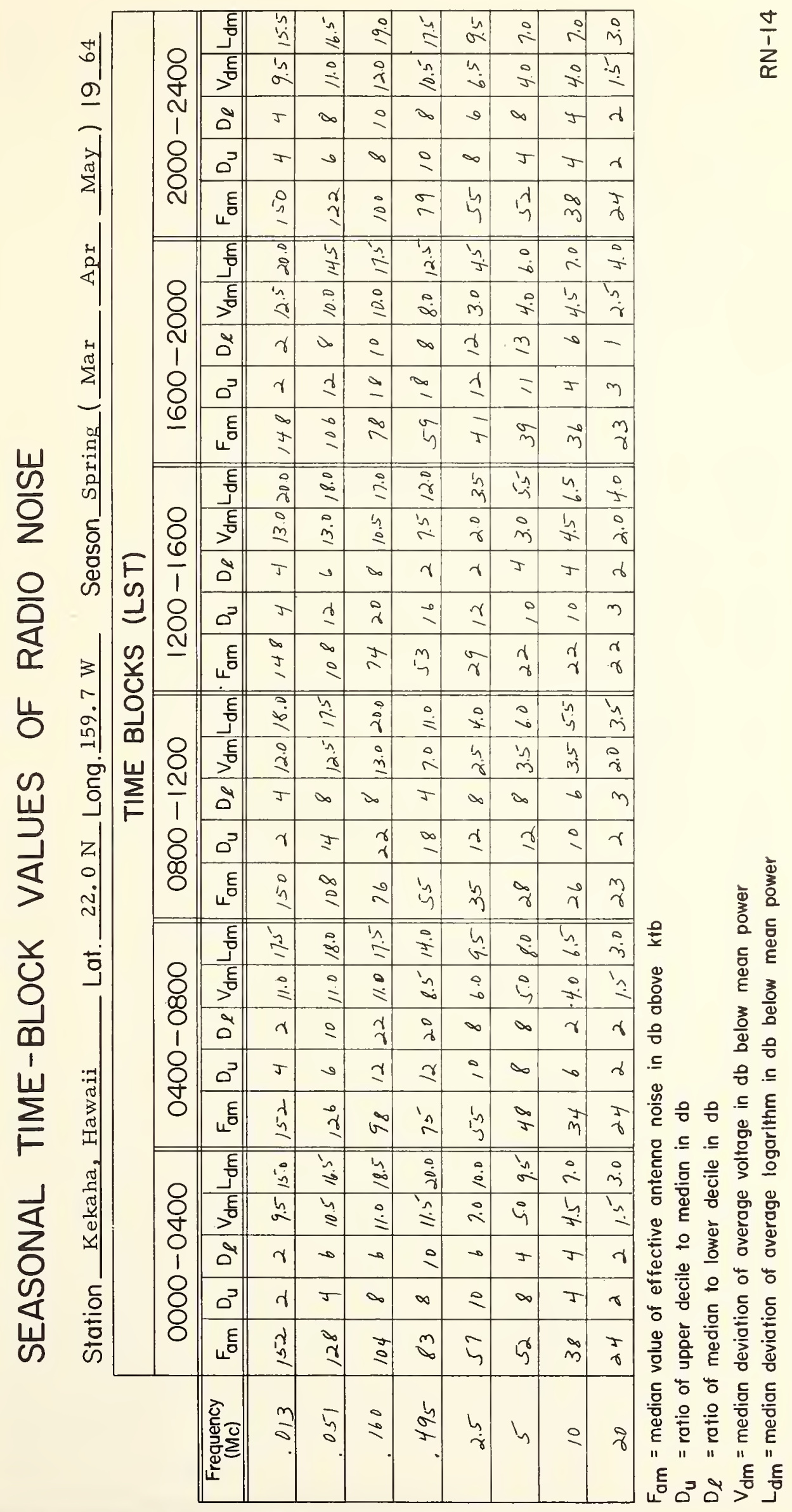




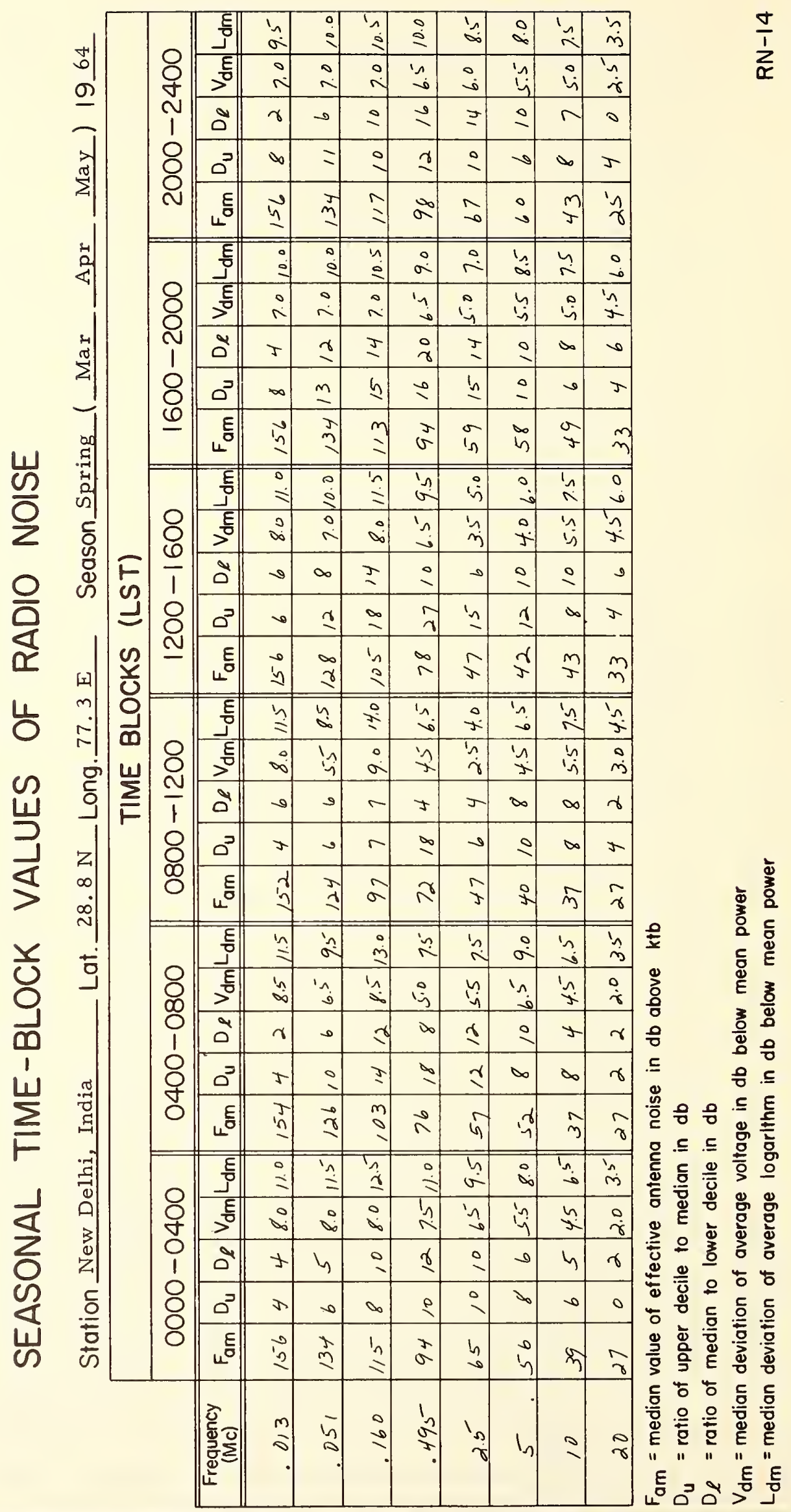




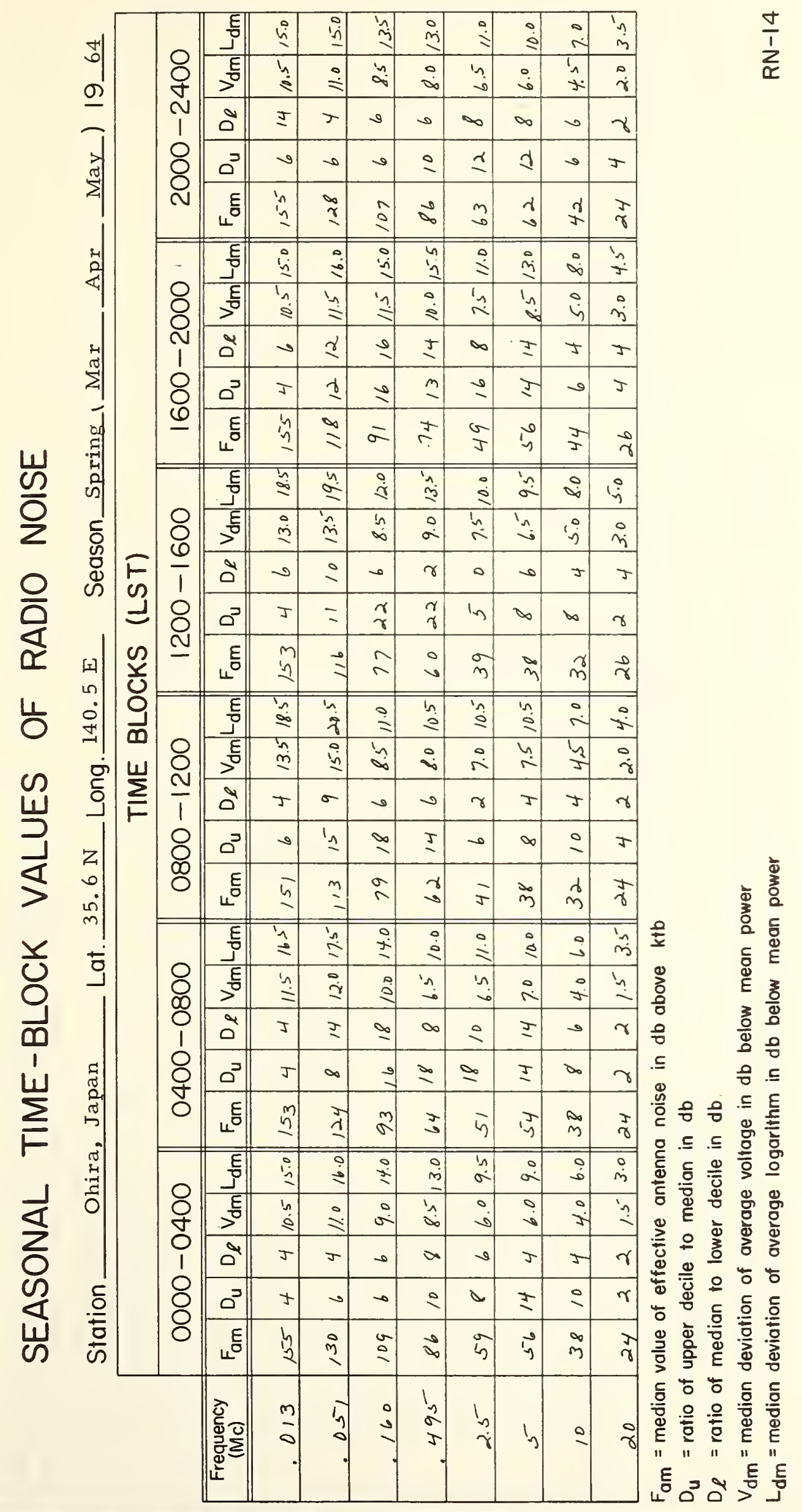




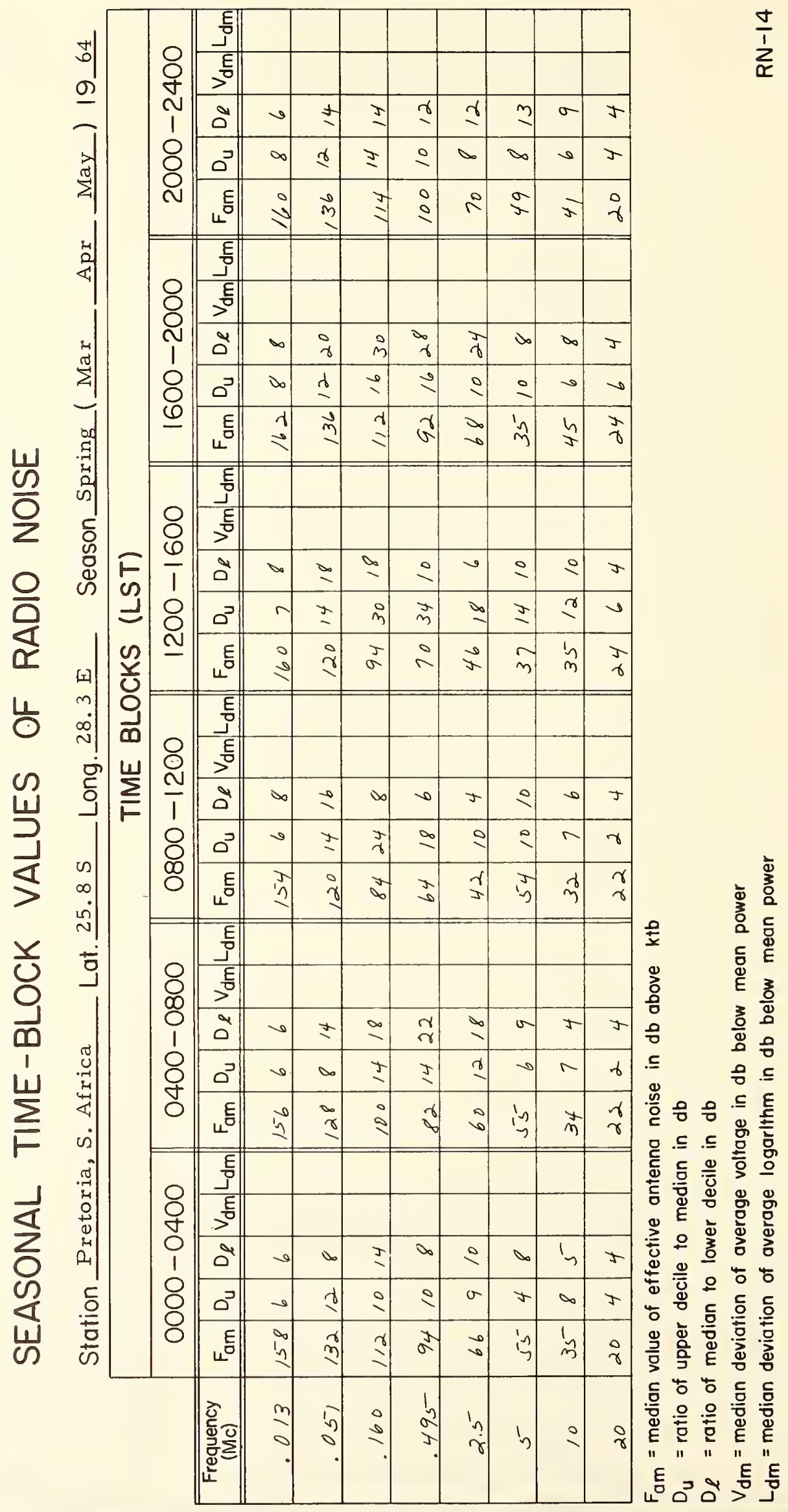




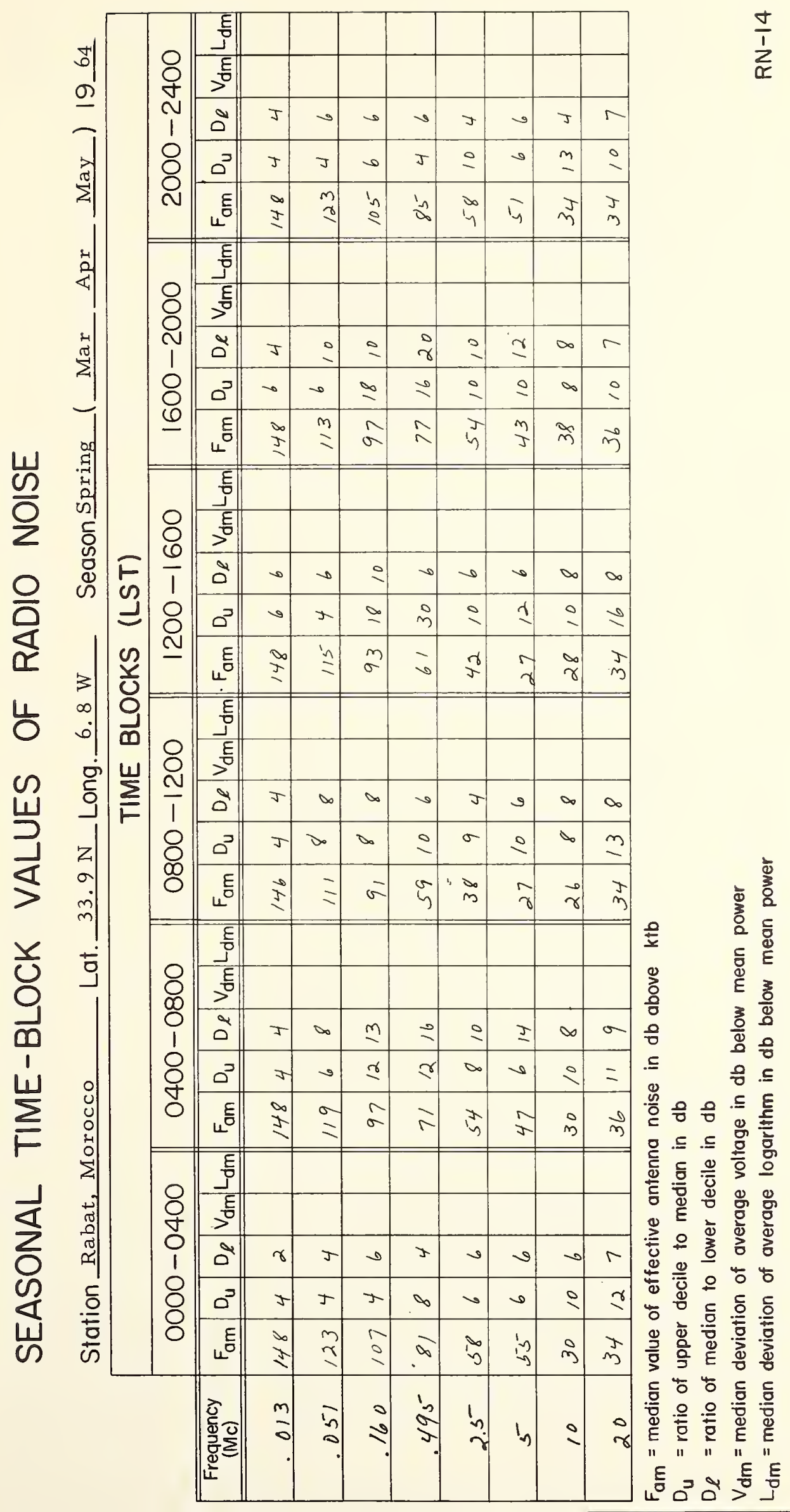




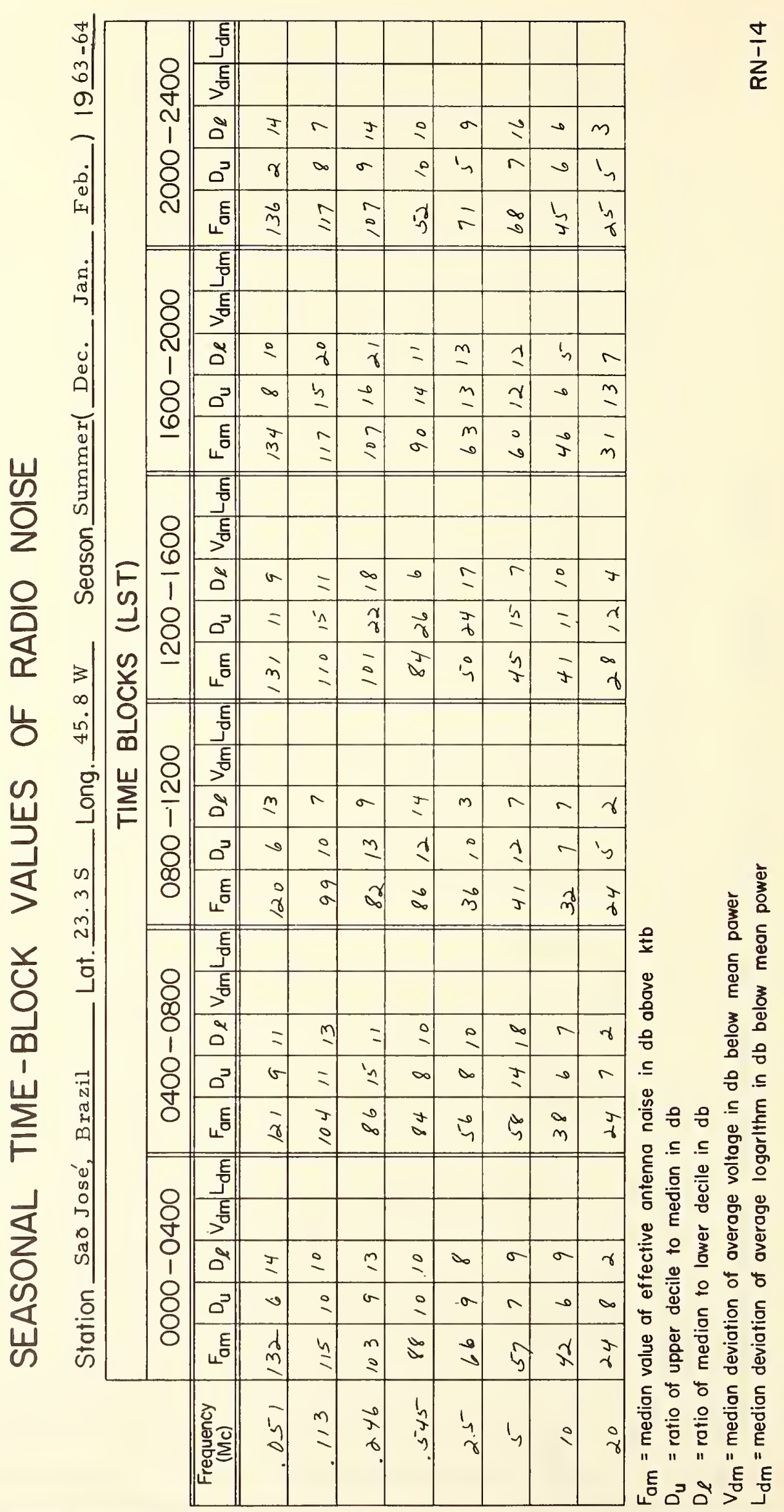




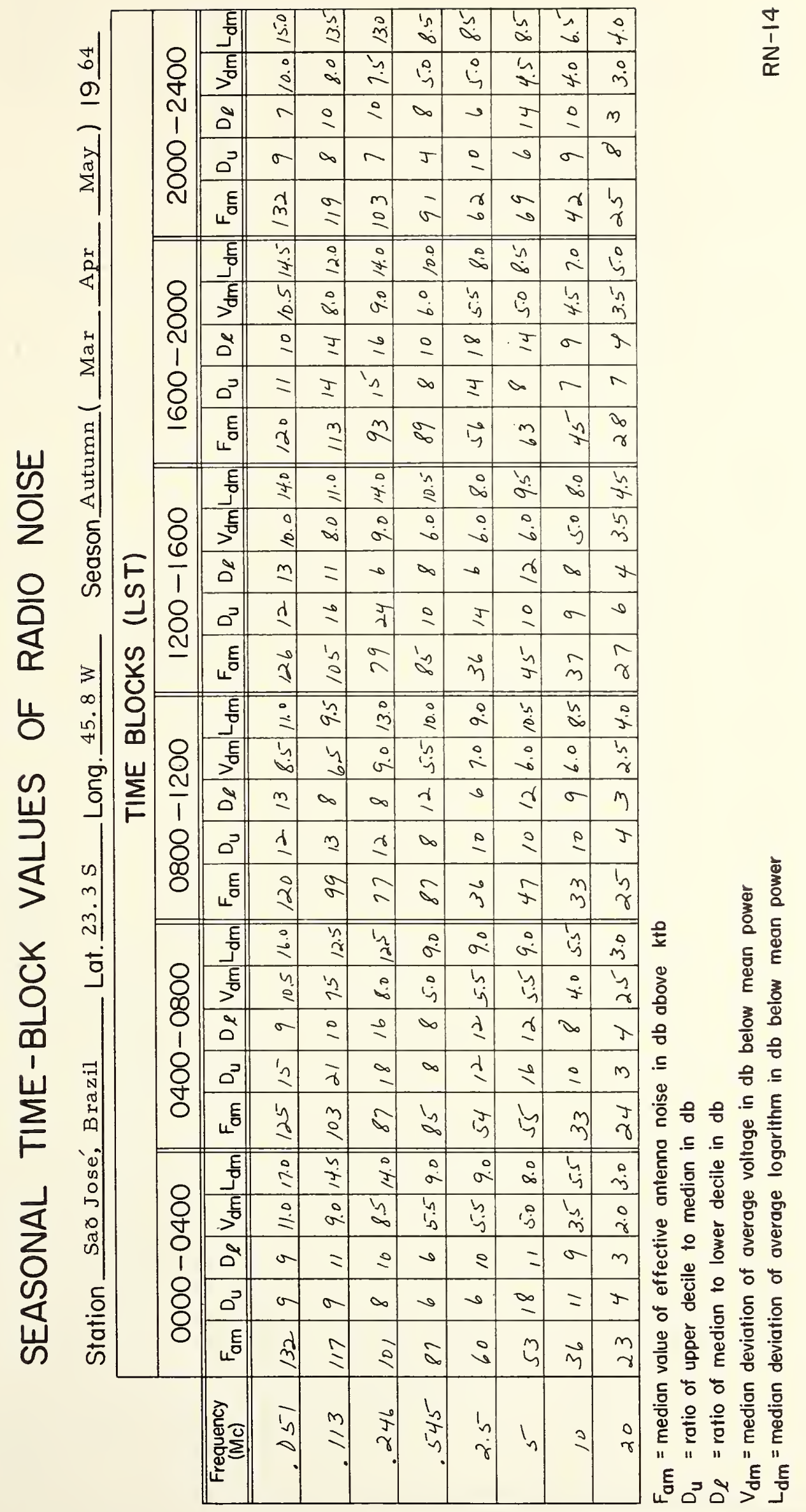




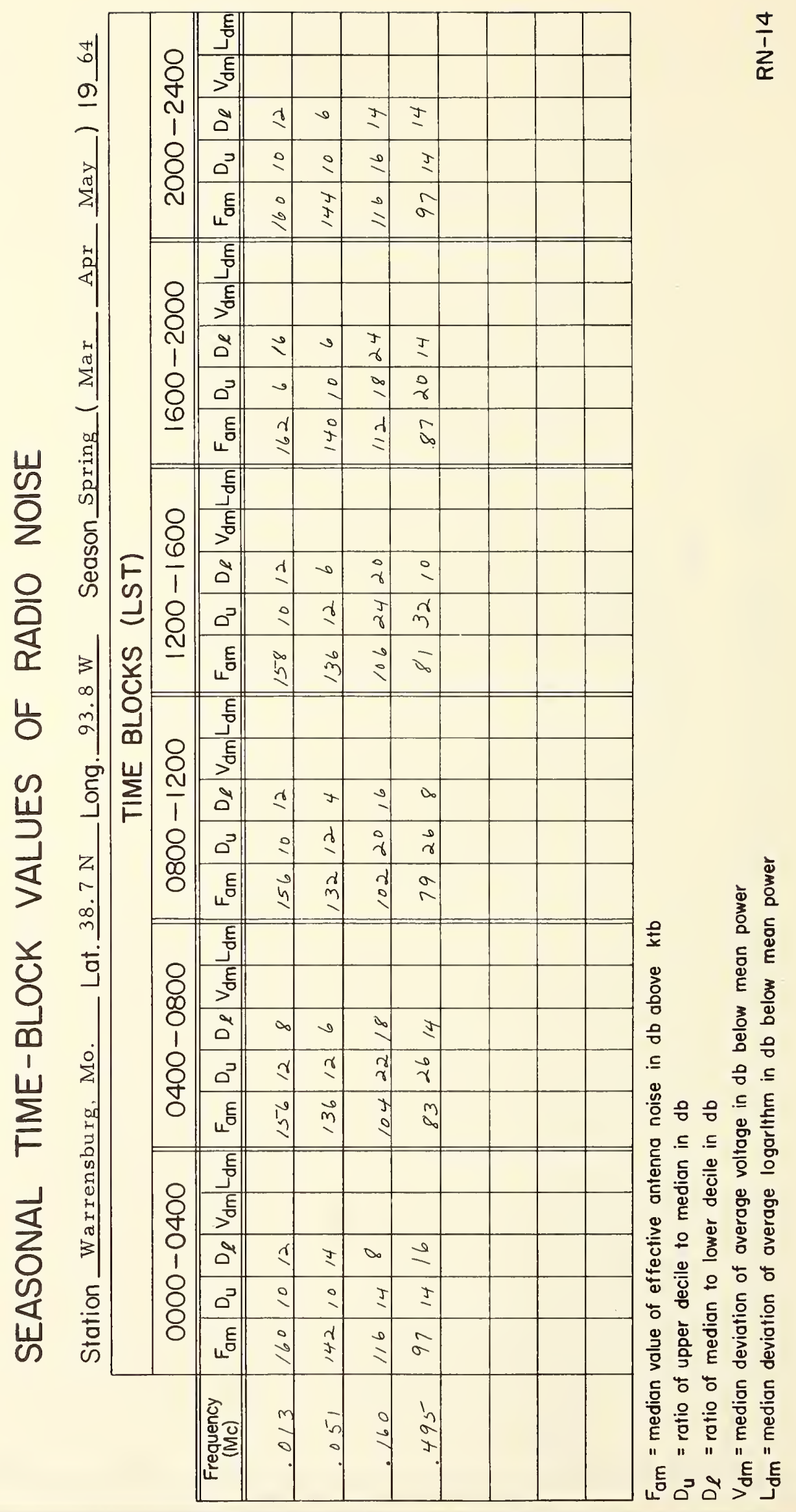





\section{U.S. DEPARTMENT OF COMMERCE}

WASHINGTON, D.C. 20230

OFFICIAL BUSINESS 